Lehrstuhl für Ernährungsmedizin der Technischen Universität

München,

Klinikum rechts der Isar,

Else-Kröner-Fresenius-Zentrum für Ernährungsmedizin

\title{
Kosten der Adipositas in der Bundesrepublik Deutschland
}

\author{
Dr. rer. pol. Klaus-Peter Knoll
}

Vollständiger Abdruck der von der Fakultät für Medizin

der Technischen Universität München zur Erlangung des akademischen Grades eines

Doktors der Medizin (Dr. med.)

genehmigten Dissertation.

Vorsitzender: $\quad$ Univ.-Prof. Dr. D. Neumeier

Prüfer der Dissertation:

1. Univ.-Prof. Dr. J. J. Hauner

2. Priv.-Doz. Dr. J. Chr. Erdmann

Die Dissertation wurde am 13.01.2010 bei der Technischen Universität München eingereicht und durch die Fakultät für Medizin am 21.07.2010 angenommen. 


\section{Bibliographische Beschreibung}

Knoll, Klaus-Peter

Kosten der Adipositas in der Bundesrepublik Deutschland

Technische Universität München, Diss.,

154 S., 60 Lit., 39 Online-Publ., 1 Interv., 10 Abb., 28 Tabellen zzgl. Anhang

Referat:

Die vorliegende Arbeit versteht sich als Dissertationsbeitrag an der Schnittstelle zwischen Medizin und Gesundheitsökonomie. Mit dem Gegenstand „Adipositas“ wird ein medizinisch relevanter Schwerpunkt bearbeitet, der bereits den Charakter einer Epidemie aufweist und scheinbar unaufhaltsam zu einem zentralen Gesundheits- und Kostenproblem hoch entwickelter Industriegesellschaften geworden ist. Die Existenz von Adipositas (BMI $\geq 30$ $\left.\left[\mathrm{kg} / \mathrm{m}^{2}\right]\right)$ wird als einer der Hauptgründe für die Zunahme chronischer Erkrankungen, v.a. des Stoffwechsels (z.B. Diabetes mellitus Typ 2) oder auch des Herz-Kreislaufsystems (z.B. Hypertonie, KHK) gesehen, mit allen Konsequenzen (z.B. Klinikaufenthalt, Arbeitsausfall, Tod), denen adipöse Personen im Verlauf ihres Lebens mit höherem Risiko als normalgewichtige ausgesetzt sind. Die Kosten, die daraus für das Gesundheitssystem entstehen, sollen in ihrer gesamten Tragweite - v.a. unter Berücksichtigung des Verursachungsaspekts von Adipositas für assoziierte Erkrankungen - beziffert werden, um zusätzlich zu den medizinischen Anforderungen zur Behandlung bzw. Vermeidung von Adipositas eine Grundlage für gesundheitspolitische Weichenstellungen zu liefern. 


\section{Inhaltsverzeichnis}

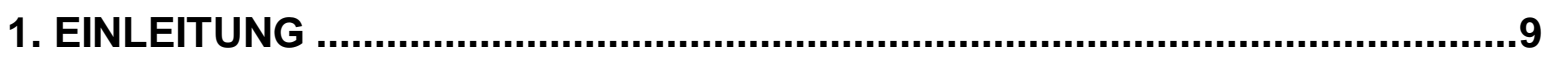

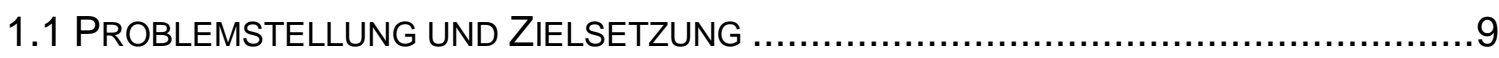

1.2 AUFBAU DER ARBEIT UND METHODISCHES VORGEHEN ....................................10

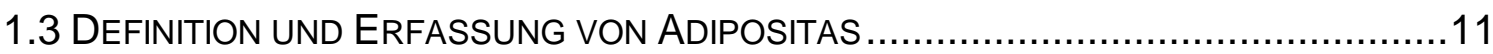

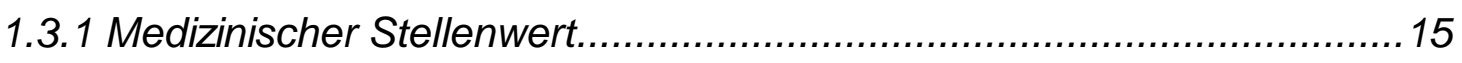

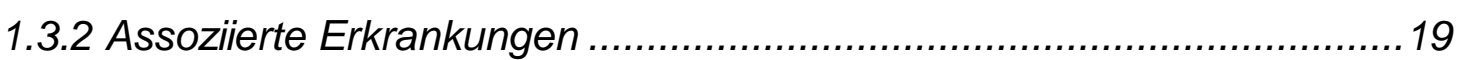

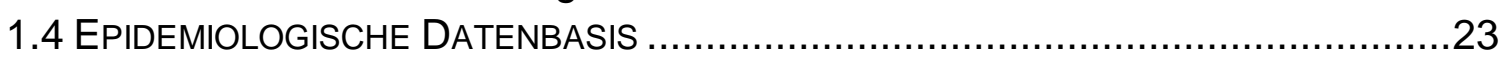

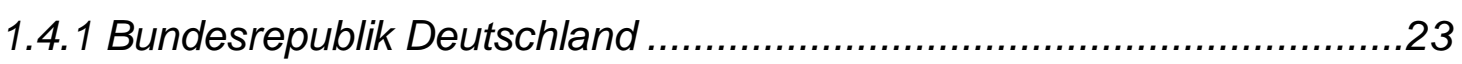

1.4.2 Vergleich zu den USA und Großbritannien ......................................26

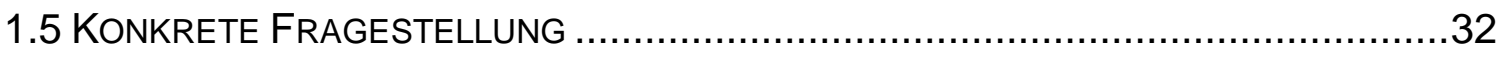

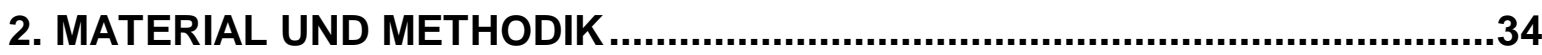

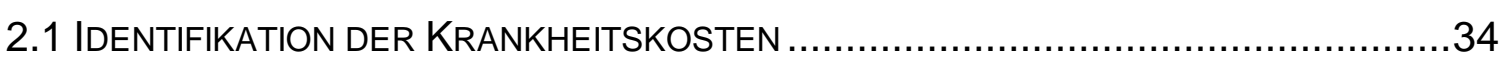

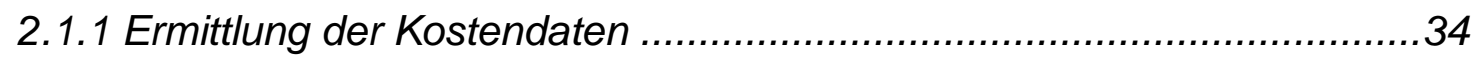

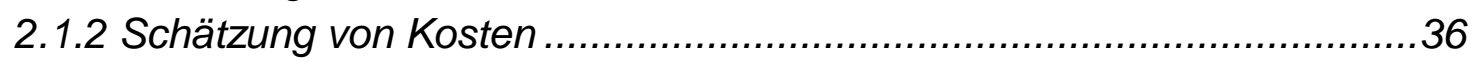

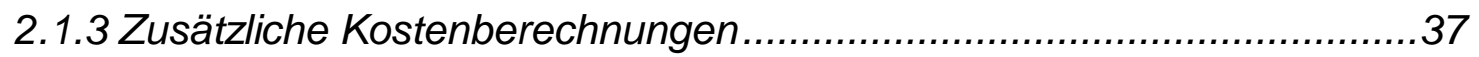

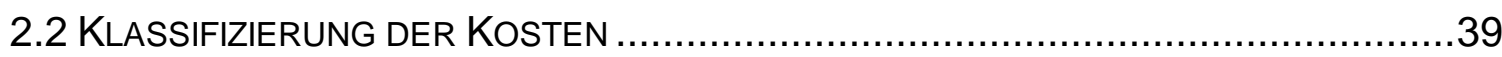

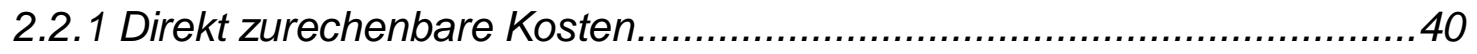

2.2.2 Indirekt zurechenbare Kosten ...................................................... 41

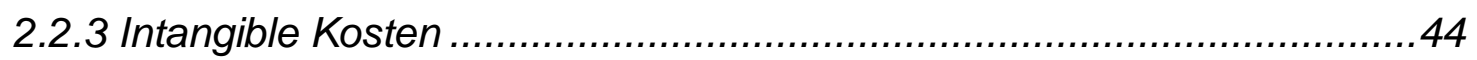

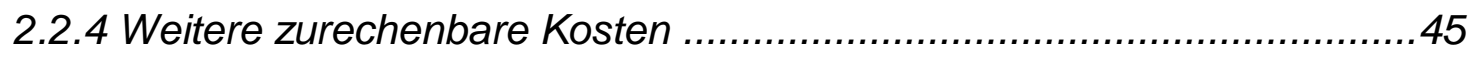

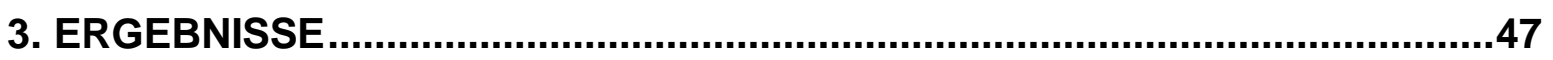

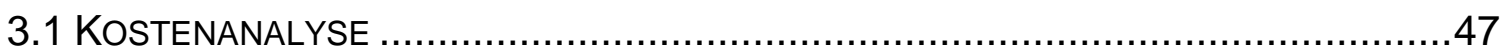

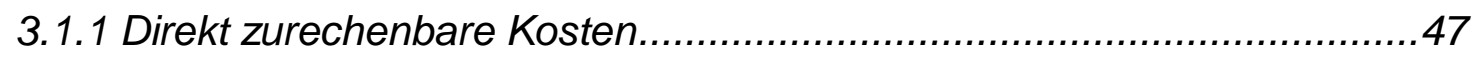

3.1.2 Direkte Kosten assoziierter Erkrankungen ..........................................60

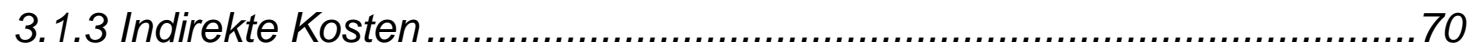

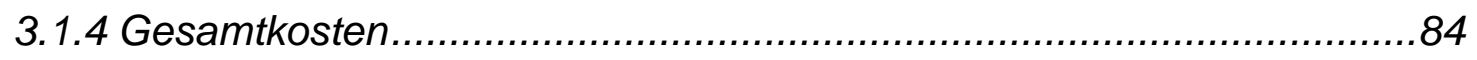

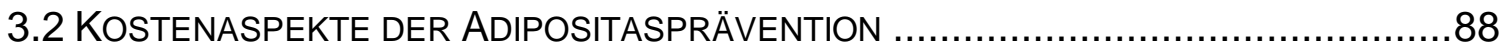

3.2.1 Initiativen zur Gesundheitsförderung und Prävention ..........................8

3.2.2 Nutzen-Kosten-Relationen der Prävention ...........................................94

3.3 ENTWICKLUNGSSZENARIEN UND HOCHRECHNUNGEN …................................96

3.3.1 Szenario 1 unter der Annahme mäßiger Entwicklungen .....................100

3.3.1 Szenario 2 unter der Annahme progressiver Entwicklungen ...............103

3.4 INTERNATIONALER VERGLEICH ZU DEN USA UND GROBBRITANNIEN..................105 
4. DISKUSSION ..............................................................................................111

4.1 METHOdISCHE ABGRENZUNG........................................................ 111

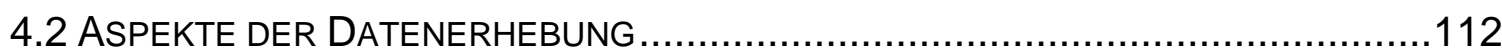

4.3 ERMITTLUNG DIREKTER KOSTEN VON ADIPOSITAS PER SE ...........................113

4.4 ERMITTLUNG DER KOSTEN ASSOZIIERTER ERKRANKUNGEN VON ADIPOSITAS ....115

4.5 ERMITTLUNG INDIREKTER KOSTEN UND BEIZUMESSENDER ANTEILE .................118

4.6 BETRACHTUNG DER GESAMTKOSTEN ...............................................119

4.7 EINORDNUNG DER ERGEBNISSE UND VERGLEICHSMÖGLICHKEITEN ..................122

4.8 HOCHRECHNUNGEN UND ZUKUNFTSPERSPEKTIVEN..................................122

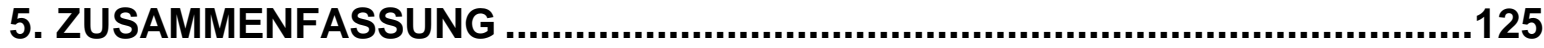

6. LITERATURVERZEICHNIS .......................................................................

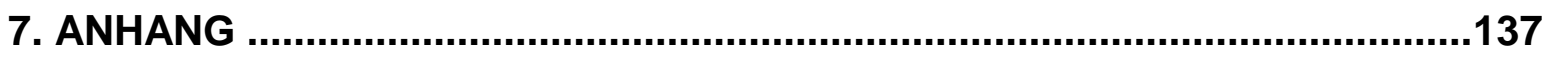

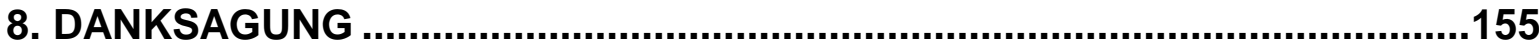




\section{Verzeichnis der Abbildungen}

Abbildung 1: Übergewicht und Adipositas im Kindes- und Jugendalter in

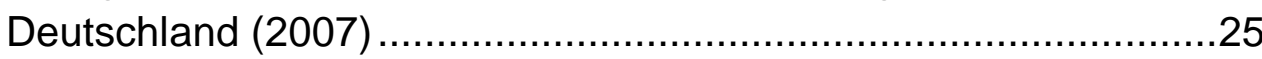

Abbildung 2: Übergewicht im Kindes- und Jugendalter in den USA .................28

Abbildung 3: Relation zwischen Übergewicht und Adipositas bei Kindern und Heranwachsenden in Großbritannien für das Jahr 2000, nach Geschlecht.

Abbildung 4: Vergleich von Übergewicht und Adipositas zwischen Deutschland, USA und Großbritannien (Erwachsene > 20 Jahre).31

Abbildung 5: Direkte Kosten der Adipositas in der Bundesrepublik Deutschland (virtuelles Bezugsjahr)

Abbildung 6: Indirekte Kosten für Arbeitsunfähigkeit, Invalidität und Mortalität bei $4 \%$ Diskontierungsrate in $1.000 €$ und $\%$

Abbildung 7: Indirekte Kosten für Arbeitsunfähigkeit, Invalidität und Mortalität bei $6 \%$ Diskontierungsrate in $1.000 €$ und $\%$

Abbildung 8: Direkte und indirekte Kosten im Vergleich bei einer Diskontierungsrate von $4 \%$ (in \%) .86

Abbildung 9: Direkte und indirekte Kosten im Vergleich bei einer Diskontierungsrate von 6\% (in \%) .86

Abbildung 10: Direkte Kosten assoziierter Erkrankungen der Adipositas in Deutschland im Vergleich verschiedener Berechnungen 


\section{Verzeichnis der Tabellen}

Tabelle 1: Klassifikation von Körpermassenindex BMI, Gewicht u. Adipositas ..12

Tabelle 2: Definition of Obesity $\left[\mathrm{kg} / \mathrm{m}^{2}\right]$ - Internationaler Vergleich ...................13

Tabelle 3: Description of Epidemiological Studies (extract overview) ...............21

Tabelle 4: Identifikation der zu Adipositas assoziierten Erkrankungen .............22

Tabelle 5: Prozentuale Verteilung von Übergewicht und Adipositas in Deutschland (2003) nach Altersgruppen und Geschlecht.

Tabelle 6: Prozentuale Verteilung von Übergewicht und Adipositas in den USA (2001-2004) nach Altersgruppen und Geschlecht.

Tabelle 7: Prozentuale Verteilung von Übergewicht und Adipositas in Großbritannien (hier nur England/ Wales) (2006) nach Altersgruppen und Geschlecht (gerundet)....

Tabelle 8: Einkaufskosten der Apotheken auf Basis der in Deutschland zugelassenen Adipositas-Medikamente Sibutramin, Orlistat und Rimonabant.

Tabelle 9: Kalkulation der stationären/ teilstationären Kosten im Falle der Adipositas-Eingriffe erster Wahl

Tabelle 10: Kalkulierte stationäre Kosten für die operative Behandlung der Adipositas (Operationen der Wahl) 55

Tabelle 11: Direkte Krankheitskosten auf Basis unterschiedlicher Erhebungen in der Übersicht .58

Tabelle 12: Bevölkerungsbezogene beizumessende Anteile (PAF) für assoziierte Erkrankungen im Zusammenhang mit Adipositas

Tabelle 13: Direkte ungewichtete und mit den bevölkerungsbezogenen beizumessenden Anteilen gewichtete Krankheitskosten der Gesundheitskostenrechnung des Bundes (2004) nach ICD 10Klassifikation

Tabelle 14: Direkte ungewichtete und mit den bevölkerungsbezogenen beizumessenden Anteilen gewichtete Krankheitskosten (in Mio. €)..69

Tabelle 15: Arbeitsunfähigkeitstage aller AOK-pflichtversicherten Arbeitnehmer 2003 ungewichtet und gewichtet mit den beizumessenden Anteilen (PAF) 
Tabelle 16: Hochrechnung der AU-Tage aller AOK-pflichtversicherten Arbeitnehmer 2003 auf die restlichen GKV in Deutschland (Basis: AOK-Zahlen)

Tabelle 17: Indirekte Kosten für Arbeitsunfähigkeit nach Geschlecht 75

Tabelle 18: Verlorene Erwerbsjahre durch Invalidität nach Geschlecht getrennt, alle Altersgruppen beginnend mit Personen „bis 39“ bis "60 und höher" (ungewichtet und gewichtet mit den beizumessenden Anteilen PAF)

Tabelle 19: Indirekte Kosten durch Invalidität (beide Geschlechter) ungewichtet und mit PAF gewichtet bei Diskontierungsraten 4\% und $6 \%$

Tabelle 20: Verlorene Erwerbsjahre durch Mortalität nach Geschlecht getrennt, alle Altersgruppen zwischen 15 und 65 Jahren (ungewichtet und gewichtet)

Tabelle 21: Indirekte Kosten durch Mortalität (beide Geschlechter) ungewichtet und mit PAF gewichtet bei Diskontierungsraten 4\% und $6 \%$

Tabelle 22: Gesamtkosten (direkte/ indirekte) der Adipositas (in $1.000 €$ ) unter Berücksichtigung der Diskontierungsraten 4\% u. 6\% (Rundungsabweichungen).

Tabelle 23: Kalkulierte Mehrausgaben für Gesundheitsförderung und Prävention der Bundesregierung

Tabelle 24: Szenario 1: Hochrechnung von Kosten für Adipositas bis ins Jahr 2020 - mäßige Entwicklung

Tabelle 25: Szenario 2: Hochrechnung von Kosten für Adipositas bis ins Jahr 2020 - dynamisierte Entwicklung .....

Tabelle 26: Unterschiede von bevölkerungsbezogenen beizumessenden Anteilen (PAF) zu Untersuchungen aus den USA und Deutschland.

Tabelle 28: Direkte Kosten selektiver assoziierter Erkrankungen ungewichtet und gewichtet und relative Anteile für Großbritannien (Angaben in Mio. $£$ bzw. $€$ und \%) 


\section{Abkürzungsverzeichnis}

$\begin{array}{ll}\text { AHT } & \text { Arterielle Hypertonie } \\ \text { AU } & \text { Arbeitsunfähigkeit } \\ \text { BAG } & \text { Bundesamt für Gesundheit (Bern, Schweiz) } \\ \text { BIP } & \text { Bruttoinlandsprodukt } \\ \text { BMAS } & \text { Bundesministerium für Arbeit und Soziales } \\ \text { BMG } & \text { Bundesministerium für Gesundheit } \\ \text { BMI } & \text { Body Mass Index } \\ \text { CDC } & \text { The Centers for Disease Control and Prevention } \\ \text { CMAJ } & \text { Canadian Medical Association Journal } \\ \text { CoDiM } & \text { Cost burden of Diabetes Mellitus } \\ \text { DALY } & \text { Disability/ Disease Adjusted Lifeyears } \\ \text { DOMS } & \text { Düsseldorfer Obesity Mortality Study } \\ \text { EPIC } & \text { European Prospective Investigation into Cancer and Nutrition } \\ \text { ERPHO } & \text { Eastern Region Public Health Obeservatory } \\ \text { ESEMeD } & \text { European Study of the Epidemiology of Mental Disorders } \\ \text { GKV } & \text { Gesetzliche Krankenversicherung } \\ \text { NICE } & \text { National Institute for Health and Clinical Excellence } \\ \text { NHS } & \text { National Health Service NHS } \\ \text { ICD } & \text { International Statistical Classification of Diseases and Related } \\ & \text { Health Problems } \\ \text { IOTF } & \text { International Obesity Task Force } \\ \text { KHK } & \text { Koronare Herzkrankheit } \\ \text { KIGGS } & \text { Kinder- und Jugendgesundheitssurvey } \\ \text { KORA } & \text { Cooperative Health Research in the Region of Augsburg } \\ \text { Mio } & \text { Million/ Millionen } \\ \text { Mrd } & \text { Milliarde/ Milliarden } \\ \text { NASH } & \text { Nicht-alkoholische Steatohepatitis } \\ \text { NCHS } & \text { National Center for Health Statistics } \\ \text { OECD } & \text { Organization of Economic Cooperation and Development } \\ \text { PAF } & \text { Population Attributibal Fraction } \\ \text { PROCAM } & \text { Prospective Cardiovascular Münster Studie } \\ \text { Reha } & \text { Rehabilitation } \\ \text { RR } & \text { Relatives Risiko } \\ \text { SGB } & \text { Sozialgesetzbuch der Bundesrepublik Deutschland } \\ \text { SVR } & \text { Sachverständigenrat zur Begutachtung der Entwicklung im } \\ & \text { Gesundheitswesen } \\ \text { DRV } & \text { Deutsche Rentenversicherung Bund } \\ \text { WHO } & \text { World Health Organization } \\ & \end{array}$




\section{Einleitung}

\subsection{Problemstellung und Zielsetzung}

„Adipositas" als Untersuchungsgegenstand einer Kostenanalyse ist gegenwärtig eine gleichsam notwendige wie komplexe Anforderung. Dies hängt zum einen mit der epidemiologischen Entwicklung zusammen, dass in Deutschland und anderen Industrieländern eine steigende Tendenz zu Übergewicht und Adipositas beobachtet wird. So liegt die Adipositas-Prävalenz in Deutschland bei über $20 \%$ der erwachsenen Bevölkerung, in machen Altersgruppen sogar über $30 \%{ }^{1}$ Auch Kinder sind zunehmend häufiger betroffen. Des Weiteren hat sich herausgestellt, dass adipöse Personen rd. 70\% und stark adipöse sogar um 3-fach höhere Kosten erzeugen als Normalgewichtige. ${ }^{2}$ Zum anderen wird der Nachweis adipöser Körperzustände allein nicht als Hauptdeterminante der Kostenentstehung gesehen, sondern in besonderem Maße die damit verbundenen erhöhten Risiken, bestimmte Krankheiten in ihrer Entstehung und Progression zu begünstigen. ${ }^{3}$

Um eine umfassende Analyse erstellen zu können ist es erforderlich, die relevanten medizinischen Kausalitäten zu identifizieren und sie in einer Weise zu bewerten, dass sie zu einem Gesamtbild aller durch Adipositas entstehenden Kosten führen. Nur über den Weg einer verursachungsgerechten Darstellung lässt sich das tatsächliche Ausmaß der ökonomischen Belastung beurteilen. Das primäre Ziel der Arbeit besteht somit darin, einen aktuellen Beitrag zur Berechnung der gesamten Kosten zu leisten, die im deutschen Gesundheitssystem (in Abhängigkeit der Datenlage) der Adipositas zuzurechnen sind. Dabei spielt gerade jener Kostenanteil eine maßgebliche Rolle, der über die Selektion und geeignete Gewichtung der Begleit- und Folgeerkrankungen ermittelt wird. Obwohl sich zahlreiche wissenschaftliche Studien unterschiedlicher Fachrichtungen mit dem Thema Adipositas befassen, sind die Aussagen und Ergebnisse über die tatsächliche gesundheitsökonomische Gesamtbelastung anhand von Kostenanalysen

\footnotetext{
${ }^{1}$ Gesundheitsberichterstattung des Bundes: Übergewicht und Adipositas Kapitel 2.6.1. http://www.gbe-bund.de/gbe10.

${ }^{2}$ Vgl.von Lengerke, T. et al (2006), S. 110ff.
} 
einerseits selten, andererseits uneinheitlich und basieren vornehmlich auf distributiven Top-down-Ansätzen. ${ }^{4}$ Vorhandene Bottom-up-Analysen, wie z.B. die sog. KORA-Studie, zielen dagegen weniger auf die Ermittlung von Gesamtkosten als vielmehr auf Kostenvergleiche zwischen Personengruppen mit unterschiedlichen Ausprägungen von Übergewicht/ Adipositas und Normalgewichtigen ab. ${ }^{5} \mathrm{Im}$ deutschsprachigen Raum konnte mit dem Studienbeitrag "Die Kosten der Adipositas in der Schweiz" eine methodisch richtungweisende, aktuelle und zugleich umfassende Erhebung auf Gesamtkostenebene identifiziert werden. ${ }^{6}$ Ansonsten setzt man sich v.a. in den USA mit Fragestellungen dieser Art auseinander. $^{7}$

Über die Ermittlung der Gesamtkosten hinaus wird eine weitere Anforderung dieser Arbeit darin gesehen, Vergleichs- und Bezugsgrößen aufzuzeigen, die eine Einschätzung der Ergebnisse aus verschiedenen Blickwinkeln heraus ermöglichen. Nicht zuletzt führen die limitierten öffentlichen Budgets im Rahmen der Gesundheitsausgaben zu einem verstärkten gesundheitspolitischen Interesse an den gegenwärtigen und zukünftigen Dimensionen der Kostenbelastung durch Adipositas.

\subsection{Aufbau der Arbeit und methodisches Vorgehen}

Der Aufbau der Dissertation folgt den üblichen Gliederungsaspekten einer wissenschaftlichen Arbeit. Im Rahmen der Einleitung wird der Untersuchungsgegenstand „Adipositas“ beschrieben und in den Themenzusammenhang eingeordnet. Zudem wird die relevante epidemiologische Situation in Deutschland dargelegt und selektiv mit anderen Nationen verglichen. Im methodischen Abschnitt erfolgt die Identifikation und Klassifikation der relevanten Kostenkriterien sowie die Festlegung der zu treffenden Annahmen für die darauf aufbauenden Berechnungen. Die Ergebnisse der Kostenanalyse werden aus Komplexitätsgründen schrittweise ermittelt und Teile der Berechnungen in den Anhang der Arbeit verlagert. Die

\footnotetext{
${ }^{3}$ Vgl. z.B. Hauner, H (2007), S. 7f.

${ }^{4}$ Siehe hierzu die Aufstellung in Tabelle 10 dieser Arbeit.

${ }^{5} \mathrm{Vgl}$. die Zusammenhänge der KORA-Studie und von Lengerke et al. (2006).

${ }^{6}$ Vgl. Schneider, H./ Schmidt, A. (2004).
} 
hierzu erforderlichen Informationen liegen nur bedingt vor und müssen teils über methodische Umwege aus verschiedenen Einzelquellen/ Studien erschlossen und weiterbearbeitet werden. Idealerweise kann man dabei auf objektive Berechnungsgrößen in direkter monetärer Form zurückgreifen, was beispielsweise mit Blick auf eine monetäre Beurteilung von Lebensqualität nicht als selbstverständlich anzusehen ist. Daher ist es an verschiedenen Stellen der Kostenberechnung erforderlich, von restriktiven Annahmen auszugehen, die entweder bestimmte Zusammenhänge ausschließen oder versuchen diese zu vereinfachen.

Neben der Unterscheidung in sog. direkte und indirekte Kostenbestandteile wird zusätzlich eine Unterscheidung zwischen Kosten für Adipositas als solches und damit verbundener, sog. assoziierte Erkrankungen getroffen, die sich an der offiziellen ICD 10-Einteilung von Erkrankungsdiagnosen orientiert. Schließlich werden alle Kostenbestandteile zu Gesamtkosten gebündelt, in ihrer Ausprägung beurteilt und eingeordnet. Nach einer Einschätzung von Kostenaspekten der Adipositasprävention werden denkbare Szenarien zukünftiger Entwicklungen des Problems der Adipositas in Deutschland aufgestellt. Mit einem kurzen internationalen Vergleich der Kostensituation endet der Ergebnisteil, dem die abschließende Diskussion folgt.

\subsection{Definition und Erfassung von Adipositas}

Der Begriff „Adipositas“ (Fettleibigkeit) bezeichnet ein definiertes Übermaß des Körperfettanteils beim Menschen. Die Einteilung bzw. Unterscheidung, wann ein Mensch als normal-, unter-, übergewichtig oder adipös bezeichnet wird, wurde im Rahmen der International Obesity Task Force (IOTF) der WHO über Richtwerte des Körpermassenindex bzw. Body Mass Index „BMl“ festgeschrieben ${ }^{8}$. Die Klassifikation wird über zueinander abgegrenzte BMI-Intervalle wie folgt vorgenommen:

\footnotetext{
${ }^{7}$ Vgl. z.B. Wolf, A. M. (2007).

${ }^{8} \mathrm{Vgl}$. International Obesity Task Force (IOTF) (1998).
} 
Tabelle 1: $\quad$ Klassifikation von Körpermassenindex BMI, Gewicht u. Adipositas

\begin{tabular}{l|l|l|}
\hline $\begin{array}{l}\text { Body Mass Index }\left[\mathrm{kg} / \mathrm{m}^{2}\right] \\
<18,5\end{array}$ & WHO Klassifikation & Deutscher Sprachgebrauch \\
\hline$\geq 18,5$ bis $\leq 24,9$ & Under weight & Untergewicht \\
$\geq 25,0$ bis $\leq 29,9$ & Normal weight & Normalgewicht \\
$\geq 30,0$ bis $\leq 34,9$ & Over weight & Präadipositas \\
$\geq 35,0$ bis $\leq 39,9$ & Obesity class I & Adipositas \\
$\geq 40,0$ & Obesity class II & Morbide Adipositas
\end{tabular}

Quelle: $\quad$ Report of WHO Consultation on Obesity (1998), in Verbindung mit Hauner, $H$. (2007).

Die Adipositas selbst wiederum ist in weitere drei Klassen (classes) unterteilt, die in Klasse I eine mäßige Adipositas, in Klasse II eine schwere Adipositas und in Klasse III eine morbide Adipositas repräsentieren. Im Rahmen der zu dieser Thematik sehr beachteten KORA-Studie ${ }^{9}$ werden die Klassen (bzw. dort Grade) II und III unter der gesonderten Bezeichnung „starke Adipositas“ zusammengefasst und von der moderaten Adipositas (Grad I) und Präadipositas (Übergewicht) abgegrenzt. ${ }^{10}$ In der medizinischen Klinik orientieren sich danach Diagnose- und Therapiemaßnahmen. Gesundheitspolitisch werden Risiken im Zusammenhang mit übermäßiger Ernährung und Richtlinien zur Prävention ebenfalls über die BMI-Klassifizierung aufgezeigt. Eine weitere praktische Relevanz des BMI zeigt sich - neben dessen systematischer, statistischer Erfassung zu Dokumentationsund Verlaufszwecken - exemplarisch in der Verwendung bei den Trägern von Krankheitskosten. Diese richten z.B. Bonuszahlungen an bestimmten, definierten BMI-Maximalwerten aus und „belohnen“ die Versicherten nur dann, wenn definier-

\footnotetext{
${ }^{9}$ Vgl. von Lengerke, T. et al. (2006). S. $110 f f$.

${ }^{10}$ Dies hängt mit den ermittelten KORA-Studienergebnissen zusammen, wonach dabei festgestellt wurde, dass die moderate Adipositas (Grad I) im Vergleich zu den erfassten Normalgewichtigen und Präadipösen - zumindest in Bezug auf die direkten medizinischen Kosten - im Mittel geringe statistische Signifikanz im Vergleich zu Personengruppen mit Adipositas Grad II und III aufwiesen.
} 
te BMI-Werte nicht überschritten werden. ${ }^{11}$ Dies ist ein klares Indiz für eine präventive Handlungsstrategie zur Vermeidung von versicherungsrisikorelevanten, wahrscheinlichen Folgekosten durch übergewichtige bzw. präadipöse und adipöse Versicherte, die seitens der Kostenträger kalkuliert werden.

Der BMI stellt für alle Anwendungen eine einfach zu handhabende Messgröße dar und kann, ohne komplizierte Diagnostik ermittelt werden. Interessanterweise und trotz der Existenz der WHO-Klassifizierung wurde die Definition von Adipositas über den BMI im internationalen Vergleich offensichtlich noch nicht einheitlich angewendet, was nachfolgende Tabelle veranschaulicht.

Tabelle 2: $\quad$ Definition of Obesity $\left[\mathrm{kg} / \mathrm{m}^{2}\right]-$ Internationaler Vergleich

\begin{tabular}{|l|l|l|l|l|l|l|l|l|}
\hline $\begin{array}{l}\text { Australia } \\
1989\end{array}$ & $\begin{array}{l}\text { France } \\
1992\end{array}$ & $\begin{array}{l}\text { Canada } \\
1997\end{array}$ & $\begin{array}{l}\text { New Zealand } \\
1991\end{array}$ & $\begin{array}{l}\text { Switzerland } \\
2001\end{array}$ & $\begin{array}{l}\text { Portugal } \\
1996\end{array}$ & $\begin{array}{l}\text { Netherlands } \\
\text { (no year) }\end{array}$ & $\begin{array}{l}\text { UK } \\
\text { (no } \\
\text { year) }\end{array}$ & $\begin{array}{l}1995 \\
\text { (n) }\end{array}$ \\
\hline$\geq 30$ & $\geq 27$ & $\geq 30$ & $\geq 30$ & $\geq 25$ & $\geq 30$ & $\geq 25$ & $\geq 30$ & $\geq 29$ \\
\hline
\end{tabular}

Quelle: $\quad$ Wolf, Anne M. (2007): Health Economics of Obesity: New Insights. Department of Public Health Sciences University of Virginia School of Medicine (Teiltabelle übernommen aus einem internen, unveröffentlichten Papier)

Heute ist die Definition von Adipositas ab einem BMI von $\geq 30$ in den westlichen Industrieländern allgemein akzeptiert. Nur in Asien besteht gemäß IOTF ein anderer Definitionsansatz, so dass für asiatische Bevölkerungsgruppen eine Anpassung erfolgen musste und Grad I Adipositas bereits für das BMI-Intervall $\geq 27,5$ bis $\leq 32,4$, der Grad II bei $\geq 32,5$ bis $\leq 37,4$ und der Grad III bereits bei $\geq 37,5$ gegeben ist. ${ }^{12}$ Einerseits ist die BMI-Klassifizierung aufgrund des direkten Zusammenhangs von Körpergewicht und Körpergröße zwar einfach zu ermitteln und zu handhaben. Andererseits ist das $\mathrm{Ma}$ jedoch zu grob, um individuelle

\footnotetext{
${ }^{11}$ Ein Beispiel für derartiges Vorgehen liefert die private Krankenversicherung AXA Colonia, die bei einem BMI innerhalb des Werteintervalls für Normlagewichtige (bis $\leq 24,9$ ) eine Bonuszahlung leistet, die bei sämtlichen Werten darüber nicht gewährt wird.

${ }^{12}$ Kossmann, B. et. al (2006): Anhang 1, Tabelle A.2 auf Grundlage einer WHO Expertise des Jahres 2004.
} 
körperliche Gegebenheiten zu berücksichtigen. Gerade für sportliche, muskulöse Personengruppen kann ein BMI von > 25 schnell erreicht werden, ohne dass mit möglicherweise erhöhten Risiken einer mit diesem BMl als übergewichtig geltende Person zu rechnen ist. Dennoch ist die Einheit „Body Mass Index“ für die Erfassung und Bewertung der dem Übergewicht folgenden Kategorie von Adipositas als durchweg tragfähig anzusehen, da in jedem Fall davon auszugehen ist, dass ab einem BMI von $\geq 30$ (WHO-Norm) die individuellen Unterschiede statistisch keine signifikante Relevanz mehr aufweisen. Unabhängig von individueller Konstitution einer Person, ist ab einem BMI $\geq 30$ aus anatomischen und physiologischen Gründen von einer Adipositas auszugehen, mit allen damit verbundenen gesundheitsrelevanten Risiken und Konsequenzen. Ggf. immer noch davon abweichende Personengruppen (z.B. Extrem-Bodybuilder oder Gewichtheber) sind als Ausnahmen anzusehen und für den Ansatz dieser Betrachtung vernachlässigbar.

Zur Vervollständigung der Zusammenhänge ist zu erwähnen, dass das wahrscheinliche Risiko für entstehende Folge- bzw. Begleiterkrankungen zusätzlich in Verbindung mit dem Fettverteilungsmuster am menschlichen Körper gesehen wird. ${ }^{13}$ Hier unterscheidet man zur Risikostratifizierung zwischen einer zentralen Fettverteilung, dem sog. „androiden Typ“ und einer vorwiegend peripheren Fettverteilung, dem sog. "gynoiden Typ“. Der androide Typ („Apfelform“) wird dabei als noch begünstigender für gesundheitliche Risiken angesehen als der gynoide Typ („Birnenform“). Zusätzlich wird diese Einteilung durch die Größe "Taillenumfang" (in $\mathrm{cm}$ ) noch weiter spezifiziert ${ }^{14}$, was aber auch v.a. aus klinischer und therapeutischer, weniger aus Kostensicht, von Relevanz ist.

In der Konsequenz sollen für den weiteren Verlauf der Arbeit und zur Bestimmung der Gesundheitskosten der Adipositas die nicht BMI-assoziierten Klassifizierungs-

\footnotetext{
${ }^{13}$ Vgl. u.a. RKI (2003): Themenheft 16 „Übergewicht und Adipositas.

${ }^{14}$ Bei Männern wird ein Taillenumfang von $\geq 102 \mathrm{~cm}$ und bei Frauen von $\geq 88 \mathrm{~cm}$ mit einem deutlich erhöhten Risiko für metabolische und kardiovaskuläre Erkrankungen in Zusammenhang gebracht. Bei einem Umfang von $\geq 94 \mathrm{~cm}$ bei Männern bzw. $80 \mathrm{~cm}$ bei Frauen wird bereits ein erhöhtes Risiko gesehen. Vgl. dazu Tabelle 1 bei Deutsche Gesellschaft für Ernährung e.V.
} 
aspekte nicht weiter berücksichtigt werden, so dass die sich anschließenden Ausführungen ausschließlich der BMI-Klassifikation, gemäß WHO-Standard, folgen und die epidemiologischen Untersuchungen an dieser Messgröße festgemacht werden. Zum einen sind entsprechende Daten zu nicht BMI-assoziierten Angaben nicht in benötigtem Umfang zugänglich, zum anderen gilt die Aufmerksamkeit der Untersuchung der Kosten für Adipositas und damit assoziierten Begleiterkrankungen, für deren Bestimmung grundsätzlich die offiziell von der WHO festgelegte Einheit des BMl als Basisbezugsgröße zugrunde gelegt wird. Eine ergänzende Berücksichtigung der Größen „individuelle Konstitution“, „zentrale Fettverteilung“ und „Taillenumfang“ würde den Umfang der Arbeit und der gestellten Anforderungen sprengen. Für die hier behandelte, kostenorientierte Fragestellung auf aggregierter Ebene wäre zudem daraus kein Erkenntnismehrwert zu erzielen.

\subsubsection{Medizinischer Stellenwert}

Die Diskussion über den medizinischen Stellenwert von Adipositas wird kontrovers geführt. Die Herangehensweise über den gesundheitsökonomischen Aspekt der Kosten - wie im Rahmen dieser Arbeit realisiert - stellt eine Möglichkeit dar, die Bewertung des medizinischen Gegenstands „Adipositas“ aus einer gesellschaftlichen Gesamtsicht vorzunehmen. Dabei spielen u.a. epidemiologische Rahmendaten, Risiken für assoziierte Begleiterkrankungen und prognostische Berechnungen eine entscheidende Rolle. Der medizinische Stellenwert wird bei dieser Betrachtungsweise weniger durch die, auf den einzelnen Patienten bezogenen, klinischen Zusammenhänge der Diagnostik oder Behandlung von Fettleibigkeit definiert, als vielmehr durch die (noch aufzuzeigende) Existenz einer signifikanten Kostenbelastung für das Gesundheitswesen und dem damit verbundenen gesundheitspolitischen Stellenwert in der Bundesrepublik Deutschland und anderen Industrienationen. ${ }^{15}$

Eine andere, ggf. ergänzende und nicht weniger relevante Beurteilung könnte durch die komplexen ernährungswissenschaftlichen Zusammenhänge gewonnen

\footnotetext{
(www.dge.de/modules.php?name=News\&file=article\&sid=711)

${ }^{15}$ Exemplarisch sollen hierbei im weiteren Verlauf Vergleichszahlen für die Länder USA und Großbritannien dargelegt werden.
} 
werden. Hierbei wäre z.B. zu hinterfragen, welche Ernährungsangebote, -gewohnheiten, -grundsätze und natürlich auch -fehler in Verbindung mit den jeweiligen Lebensumständen der Menschen dazu führen, dass die Bevölkerungen in Deutschland und anderen Industriestaaten mit dem Problemkomplex der Adipositas zu kämpfen haben. Es existiert eine Reihe von weiteren Untersuchungen, die sich neben den klinischen, gesundheitsökonomischen oder ernährungswissenschaftlichen Perspektiven, dem epidemieartigen Bevölkerungsproblem und Gegenstand Adipositas auch aus soziologischer oder verhaltenspsychologischer Sicht nähern. ${ }^{16}$ Wie es bei allen medizinischen Phänomenen mit epidemieartigen Ausmaßen der Fall ist, erfordert auch das Thema Adipositas notwendigerweise eine besondere Beachtung und wird gerade deswegen im Interesse aller Wissenschaftsbereiche liegen, die sich mit Entwicklungen dieser Art und Ausprägung befassen. Die Darstellung aus Kostensicht kann nur am Rande auf diese Zusammenhänge eingehen.

Ein weiterer zentraler, wie zugleich brisanter Aspekt zum medizinischen Stellenwert von Adipositas ist die Frage, ob es sich bei der Adipositas um eine definierte Krankheit handelt oder nicht. Diese Fragestellung ist aus medizinischer Sicht natürlich von allergrößtem Interesse, da sich mit der Existenz einer Krankheit klinische, therapeutische, präventive und alle darüber hinausgehenden medizinischen Konsequenzen verknüpfen, die zur Erforschung, Behandlung und im Idealfall Heilung dieser „Erkrankung“ führen. Genauso interessiert sich die Gesundheitsökonomie für die Klärung dieser Frage, da im Zusammenhang mit Adipositas Kosten entstehen, die erfasst, zugewiesen und ggf. von dem Gesundheitssystem und ihren Trägern aufgebracht werden müssen. Da allerdings bereits für den Begriff „Krankheit“ keine einheitliche Definition vorliegt, sondern im Rahmen des SGB V lediglich ein Konsens darüber herrscht, Krankheit im Sinne eines regelwidrigen Zustands des Körpers, Geistes oder der Seele aufzufassen, welcher medizinische Maßnahmen mit dem Ziel erforderlich macht, Schmerzen oder Beschwerden zu vermeiden, ist auch die Beurteilung der Adipositas als Krankheit an diesem existierenden Konsens zu messen. Die Deutsche Adipositas-Gesell-

\footnotetext{
${ }^{16}$ Vgl. z.B. Huth, K., Pudel V. (Hrsg.) (1997) oder auch Zielke, M., Mark, N. (Hrsg.) (1990).
} 
schaft e.V. hat sich, im Rahmen einer Änderung des Arzneimittelgesetzes und des darin festgelegten Ausschlusses von bestimmten Medikamentenwirkstoffen zur Bekämpfung von Fettleibigkeit, intensiv mit der Fragestellung des Krankheitswertes von Adipositas befasst. ${ }^{17}$ Trotz zahlreicher Formulierungen, Einschätzungen und Beschlüsse, Adipositas als entweder "chronische Krankheit" oder "Krankheit im krankenversicherungsrechtlichen Sinne" anzusehen, existiert keine durchgängig einheitliche Meinung in der Gesellschaft, Adipositas grundsätzlich als Krankheit aufzufassen. ${ }^{18}$ In einer breit angelegten Untersuchung am Stiftungslehrstuhl für Medizinmanagement der Universität Duisburg-Essen aus dem Jahr 2006 wurde die Frage, ob Adipositas eine Krankheit sei, ebenfalls als Untersuchungsgegenstand herangezogen. ${ }^{19}$ Als Ergebnis dieser Studie wurde ermittelt, dass eine überwiegende Anzahl von mehr als 40 nationalen und internationalen Leitlinien, Adipositas als eine Krankheit bezeichnet. Ein geringerer Teil schätzt Adipositas zumindest als Risikofaktor für schwerwiegende Erkrankungen ein und weist auf die gesellschaftliche Bedeutung der steigenden Prävalenz von Adipositas hin. ${ }^{20}$

Grundsätzlich sieht das SGB für jeden gesetzlich Versicherten einen Anspruch auf eine Krankenbehandlung vor, sofern diese erforderlich ist, um eine Krankheit zu erkennen, zu heilen oder ihre Verschlimmerung (ggf. Arbeitsunfähigkeit) zu vermeiden bzw. Beschwerden im Zusammenhang mit dieser Erkrankung zu lindern. Gilt die Adipositas in o.g. Sinn demnach nicht als anerkannte Krankheit im Rahmen der aktuellen Klassifizierung nach ICD 10, würden „Krankheitskosten“ auch nicht über das (gängige) Leistungsspektrum der Krankenkassen als Kostenträger abgedeckt. Die im Zusammenhang mit der Adipositas stehenden, assoziierten (Ko-)Morbiditäten (z.B. Diabetes Mellitus Typ 2 oder AHT) sind in ihrer Anerkennung als eigenständige Krankheiten zwar unstrittig und somit auch die Kostenübernahme durch die jeweiligen Kostenträger. Die Schwierigkeit hierbei liegt jedoch zum einen in dem Nachweis, inwieweit Adipositas derartige Erkran-

\footnotetext{
${ }^{17}$ Deutsche Adipositas-Gesellschaft (2006), S.2-6.

${ }^{18}$ Ebenda S. 3.

${ }^{19}$ Kossmann, B. et. al. (2006).

${ }^{20}$ Ebenda, S. 5 und S. 10.
} 
kungen auslöst bzw. in ihrer Entstehung oder Progression fördert und somit in der initialen Kausalfolge zu sehen ist. Zum anderen muss - nach ggf. gelungenem Nachweis eines direkten kausalen Zusammenhangs - ein (relativer) korrelierender Kostenanteil ermittelt werden, welcher die Begleit- oder Folgeerkrankungen aufgrund einer bestehenden Adipositas beziffert. Diese Aspekte spielen auch im Zusammenhang mit dieser Arbeit eine zentrale Rolle.

Eine eindeutige Klärung der dahingehend aufgeworfenen Fragen ist im Rahmen dieser Arbeit weder möglich noch angestrebt. Zusammenfassend spielt es eine, für die im weiteren Verlauf vorgenommenen Kostenberechnungen, untergeordnete Rolle, ob Adipositas im Grundsatz als Krankheit gilt oder nicht. Bei weitem wichtiger ist die Belegung der These, dass durch Adipositas in Deutschland umfangreiche Gesundheitskosten entstehen und somit die Frage, in welcher Höhe sich diese beziffern und ob - kritisch beurteilt - die bestehende statistische Datenerfassung überhaupt in der Lage ist, alle relevanten Kosten, die aufgrund von oder im Zusammenhang mit Adipositas anfallen, vorzuhalten. Rein sachlich aus der Kostensicht betrachtet ist zu diesem Zeitpunkt der Arbeit festzustellen, dass ein medizinischer Stellenwert von Adipositas im Rahmen des ICD 10 Klassifizierungszifferintervalls E65 bis E68 gegeben ist und sie im Rahmen dieser Diagnosen als Abrechnungs- und somit als Kostengegenstand faktisch berücksichtigt wird, wenngleich - wie sich zeigen wird - nur ein Teil der Leistungen von den Kostenträgern übernommen wird. Diese ICD-Ziffern wiederum befinden sich im übergeordneten Ziffernintervall E00 bis E90, das für „Endokrine, Ernährungsund Stoffwechselkrankheiten" codiert ist. ${ }^{21}$ Die noch konkret zu definierenden assoziierten Erkrankungen sind unter den verbleibenden ICD 10 Ziffern ausfindig zu machen und verursachungsgerecht den Kosten der Adipositas zuzuweisen. Es ist davon auszugehen, dass durch die Identifikation und risikoorientierte Zuordnung dieser Erkrankungen ein wesentlicher, der Adipositas zurechenbarer Kostenanteil zu berücksichtigen sein wird.

\footnotetext{
${ }^{21}$ Statistisches Bundesamt (2007): Statistisches Jahrbuch 2007, Abschnitt 9 Gesundheitswesen. Siehe insbesondere Abschnitt 9.9, S. $255 \mathrm{ff}$.
} 


\subsubsection{Assoziierte Erkrankungen}

Eine isolierte Betrachtung von Adipositas würde sowohl aus medizinischer als auch aus gesundheitsökonomischer Sicht nicht den Anforderungen an eine tragfähige Analyse genügen, da ansonsten ein wesentlicher Untersuchungsaspekt außer Acht gelassen würde, nämlich jener Anteil von Erkrankungen, der in engem, direkten oder indirekten Zusammenhang mit einem übermäßigen Körperfettanteil zu sehen ist. Diesen als sog. assoziierte Erkrankungen bezeichneten Gesundheitsdefiziten wird, aufgrund von adipösen Körperzuständen der betroffenen Patienten, eine entscheidende Bedeutung zugesprochen, so dass ein übermäßiger Körperfettanteil als anteilig ursächlicher Grund für das Entstehen dieser Erkrankungen verantwortlich gemacht wird. Über das Zustandekommen dieser Kausalitäten existiert eine Reihe wissenschaftlicher Beiträge, die in erster Linie über Langzeitstudien zu mittlerweile anerkannten Aussagen kommen. ${ }^{22}$ Insbesondere zu kardiovaskulären Erkrankungen und damit verbundenen höheren Mortalitätsrisiken, die sich auf Ernährung, Ernährungsverhalten, Adipositas und andere Risikofaktoren (z.B. Alkohol, Rauchen) zurückführen lassen, finden sich Arbeiten. ${ }^{23}$ Dabei sollte allerdings nicht übersehen werden, dass die Schwierigkeit der eindeutigen Zuordnung eines einzelnen Risikofaktors zu einer bestimmten assoziierten Erkrankung für alle diesbezüglichen Studien besteht. Es existieren neben Einzelrisiken (z.B. Ernährungsverhalten) Risikoprofile mit mehreren Risikofaktoren (z.B. Bewegungsmangel und Ernährungsverhalten), welche ein Erkrankungsrisiko determinieren.

Nichtsdestoweniger kommt beispielsweise das Robert Koch-Institut zu dem Ergebnis, dass auch wenn keine einfache ursächliche Beziehung anzunehmen sei, weil Begleit- und Folgeerkrankungen z.B. auch von Bewegungsmangel und vom Tabakkonsum beeinflusst würden, man im Rahmen eines multifaktoriellen Bedingungsmodells heute doch von einer besonderen kausalen Bedeutung der Adipositas für die Entstehung dieser Krankheiten ausgehe. ${ }^{24}$ Es ist daher sowohl

\footnotetext{
${ }^{22}$ Vgl. z.B. Stampfer M. J., Hu F. B., Manson J. E. et al. (2000), S. 16-22 oder auch World Health Association (2003) Technical Report Series 916.

${ }^{23}$ Khaw K. T. et al (2008).

${ }^{24}$ Benecke, A./ Vogel, H. (2005): Gesundheitsberichterstattung des Bundes Heft 16.
} 
als unumgänglich wie auch konsequent anzusehen, gleichzeitig Kosten für Adipositas und die damit assoziierten Erkrankungen zu dokumentieren und in ihrer Höhe zu beziffern, da nur auf diese Weise wirtschaftliches Ausmaß und gesellschaftliche Bedeutung des gesamten Themenkomplexes der Adipositas beurteilt und ggf. erforderliche Maßnahmen getroffen sowie eine öffentliche Diskussion darüber geführt werden können.

Es gilt daraufhin zu klären, welches konkret die Begleit- oder Folgeerkrankungen - kurz die assoziierten Erkrankungen - sind und welche Prioritäten man ihnen im Zusammenhang mit Adipositas zuordnen kann. Sicherlich wäre es nicht zielführend, an dieser Stelle alle entfernt denkbaren Kausalitäten und mehr oder weniger verknüpften Krankheitsbilder anzuführen. Vielmehr gilt es, die dominierenden Krankheiten zu identifizieren, für die der assoziierte Zusammenhang mit Blick auf die Kostenberechnung zum einen am wahrscheinlichsten, zum anderen am gravierendsten ist. Unter Berücksichtigung dieser Aspekte stehen nachfolgende Zusammenhänge zwischen Adipositas und Gesundheitsfolgen im Brennpunkt des Interesses.

Den Bestimmungen und Konventionen folgend sind die zu Adipositas assoziierten Erkrankungen an der Relation zwischen der definierenden Größe des BMI von Betroffenen und der in der Folge auftretenden Prävalenz der Begleit- oder Folgeerkrankungen festzumachen. V.a. in den USA wurden hierzu umfangreiche, wegweisende Arbeiten durchgeführt, welche als ein wesentliches Fundament dieses Forschungsgebiets anzusehen sind und in darauf aufbauenden Metaanalysen Berücksichtigung finden: 
Tabelle 3: $\quad$ Description of Epidemiological Studies (extract overview)

\begin{tabular}{|c|c|c|}
\hline Study Population & Type of Study & $\begin{array}{l}\text { Disease States } \\
\text { Evaluated }\end{array}$ \\
\hline $\begin{array}{l}\text { Nurses' Health Study and } \\
\text { Health Professional } \\
\text { Follow-up Study }\end{array}$ & $\begin{array}{l}\text { Prospective Cohort } \\
\text { Studies of Women and Men }\end{array}$ & $\begin{array}{l}\text { Type } 2 \text { Diabetes } \\
\text { Coronary Heart Disease } \\
\text { Stroke } \\
\text { Hypertension } \\
\text { Gallstones }\end{array}$ \\
\hline $\begin{array}{l}\text { Frahamingham Heart } \\
\text { Study \& Offpring Study }\end{array}$ & Prospective Cohort Study & Congestive Heart Failure \\
\hline $\begin{array}{l}\text { National Health and Nutrition } \\
\text { Examination Survey I } \\
\text { (NHANES I) }\end{array}$ & $\begin{array}{l}\text { Cross Sectional, Descriptive } \\
\text { Study }\end{array}$ & Ostheoarthritis \\
\hline Cancer Prevention Study II & Prospective Cohort Study & $\begin{array}{l}\text { Cancer (e.g. Esophageal, } \\
\text { Colorectal, Cervical etc.) }\end{array}$ \\
\hline
\end{tabular}

Quelle:

McCusker, M. E, Finkelstein, E. A. et. al.(2004): Disease Specific Costs of Obesity), Table 1, Seite 28.

Auch Studien aus Deutschland, wie die Münster Heart Study (PROCAM), die Augsburger KORA-Studie oder auch die Düsseldorfer Obesity Mortality Study (DOMS) und aus der Schweiz (Abschlussbericht BAG) finden in diesem Zusammenhang Beachtung. ${ }^{25}$ Leider werden Kostenberechnungen nur in einem kleineren Teil der Studien aus dem deutschsprachigen Raum vorgenommen, so dass die Verfügbarkeit - insbesondere von gesamtbevölkerungsbezogenen Kosten unterrepräsentiert oder teils nicht gegeben ist. Hingegen leisten viele Studien einen wesentlichen Beitrag zur Identifikation der wichtigsten assoziierten Erkrankungen von Adipositas. Bündelt man diese Erkenntnisse und ordnet den Erkrankungen die zugehörigen ICD 10-Ziffern zu, kann nachstehende Selektion vorgenommen werden:

\footnotetext{
${ }^{25}$ Assmann, G. et. al. (1997), S. 237-242 sowie Bender, R. et. al (1999), S. 1498-1504. Der für das Schweizer Bundesamt für Gesundheit (BAG) in Bern verfasste Abschlussbericht für das von Schneider, H./ Schmid, A. (2004), stellt eine zentrale Bezugsstudie auch für diese Arbeit dar.
} 
Tabelle 4: $\quad$ Identifikation der zu Adipositas assoziierten Erkrankungen

\begin{tabular}{l|l}
\hline Assoziierte Erkrankung & Ziffern nach ICD 10 \\
\hline Hypertonie/ Hochdruckkrankheit & I $10-$ I 15 \\
\hline Diabetes Mellitus (v.a. Typ 2) & E 11 - E 14 \\
\hline Ischämische Herzkrankheiten/ Herzinsuffizienz & I $20-$ I 25 und I 50 \\
\hline Schlaganfall & I $63-$ I 64 \\
\hline Neubildungen/ Bösartige Neubildungen & C $00-$ C 97 \\
\hline Krankheiten der Gallenblase, -wege und des Pankreas & K $80-$ K 87 \\
\hline Arthrose (v.a. Gonarthrose/ Coxarthrose) & M $15-$ M 17 \\
\hline Psychosoziale Komplikationen (Depression) & F $32-$ F34 \\
\hline Schlafstörungen/ Schlafapnoe & G 47
\end{tabular}

Quelle: $\quad$ Eigene Zusammenstellung unter Berücksichtigung o.g. Studien sowie der Zusammenstellung in Benecke, A./ Vogel, H. (2005): Übergewicht und Adipositas a.a.O., in Verbindung mit Statistischem Jahrbuch 2007, S. 255 ff und ICD 10 Band I WHO-Ausgabe, Version 2006.

Bei der expliziten Kostenbestimmung wird eine weitergehende Betrachtung und Konkretisierung der Erkrankungen im Zusammenhang mit Adipositas erforderlich sein. Insbesondere die einzelnen Krebserkrankungen sind genauer zu selektieren, da davon ausgegangen werden muss, dass die Bestimmung der relativen Risiken im Zusammenhang mit der Entstehung bei Adipositas je nach Art der bösartigen Neubildung variieren werden. Der Grad einer Aufschlüsselung der Krankheiten wird neben medizinischen Aspekten zusätzlich durch die Verfügbarkeit von statistischem Datenmaterial limitiert. Dies gilt es kritisch, v.a. für geplante Vergleiche zu der Kostensituation in den USA und Großbritannien zu bedenken, da eine Datenerhebung nach einheitlichen Grundsätzen nicht durchgängig zu erwarten ist. Des Weiteren werden o.g. Erkrankungen lediglich als die in diesem Themenkomplex wichtigsten Folge- und Begleiterkrankungen gesehen, ohne innen einen Anspruch auf allumfassende Vollständigkeit oder vergleichbare Relevanz in anderen denkbaren Zusammenhängen zusprechen zu können. 


\subsection{Epidemiologische Datenbasis}

\subsubsection{Bundesrepublik Deutschland}

In der Bundesrepublik Deutschland werden demographische Bevölkerungsgrunddaten über den Weg von Volkszählungen bzw. Mikrozensus-Berechnungen erhoben und zyklisch aktualisiert. ${ }^{26}$ Epidemiologische Daten zu Übergewicht und Adipositas werden in der Gesundheitsberichterstattung des Bundes erfasst und finden sich auch in Studien der OECD und WHO wieder. ${ }^{27}$ Im Falle von Adipositas sind die Berechnungen auf die folgenden relativen Bevölkerungsanteile erwachsener Personen zu beziehen:

Tabelle 5: $\quad$ Prozentuale Verteilung von Übergewicht und Adipositas in Deutschland (2003) nach Altersgruppen und Geschlecht.

\begin{tabular}{|l|r|r|r|r|}
\hline $\begin{array}{l}\text { Altersgruppen } \\
\text { In Intervallen }\end{array}$ & $\begin{array}{c}|c| \\
\text { Übergewicht } \\
\text { [BMI 25-29,9] }\end{array}$ & $\begin{array}{c}\text { Adipositas } \\
\text { [BMI } \geq 30]\end{array}$ & $\begin{array}{r}\text { Übergewicht } \\
\text { [BMI 25-29,9] }\end{array}$ & $\begin{array}{c}\text { Adipositas } \\
\text { [BMI } \geq 30]\end{array}$ \\
\hline $18-29$ & 28,9 & 3,7 & 16,2 & 4,0 \\
\hline $30-39$ & 46,4 & 13,9 & 26,4 & 11,0 \\
\hline $40-49$ & 53,1 & 19,9 & 32,3 & 15,3 \\
\hline $50-59$ & 53,2 & 25,3 & 42,0 & 15,4 \\
\hline $60-69$ & 62,1 & 22,8 & 46,3 & 33,0 \\
\hline Ab 70+ & 58,2 & 22,8 & 45,7 & 32,2 \\
\hline
\end{tabular}

Quelle: Gesundheitsberichterstattung des Bundes - Übergewicht und Adipositas, Kapitel 2.6.1 [Gesundheit in Deutschland, 2006], Tabelle zu Abbildung 2.6.1. basierend auf Mikrozensus 2003 und Telefonischem Gesundheitssurvey 2003.

Trifft man eine generelle Aussage über alle Altersgruppen der erwachsenen Bevölkerung (in 2006 rund 66 Mio. > 20 Jahre von insgesamt 82,4 Mio. Einwohnern), so ist mehr als die Hälfte aller Personen übergewichtig (und adipös) und rund ein Fünftel (etwa 13,2 Mio.) gelten als adipös (ab BMI $\geq 30$ ). Dabei gilt es zu

${ }^{26}$ Unter Mikrozensus wird jene offizielle Repräsentativstatistik verstanden, die der laufenden Beobachtung der bevölkerungs- und erwerbsstatistischen Daten zwischen den großen Volkszählungen dient (aktuell vorliegend aus dem Jahr 2005).

$27 \mathrm{Vgl}$. Gesundheitsberichterstattung des Bundes, Heft 16 Übergewicht und Adipositas in Verbindung mit den nachfolgenden Fußnoten 28 bis 30. 
bedenken, dass die Kostenentstehung durch und die -verteilung auf die betroffenen Personengruppen wesentlich in Abhängigkeit von der Existenz und dem Komplexitätsgrad der mit Adipositas assoziierten Erkrankungen zu sehen ist. Auf diese Zusammenhänge wird im Rahmen der Kostenermittlung weiter eingegangen.

Je nach Fragestellung können durch die Bildung von Altersgruppen z.B. entweder Krankheitskosten nur für Patienten bestimmter Intervalle aufgezeigt oder ggf. die Kosten einer Erkrankung über alle Patienten und Altersgruppen berechnet werden. Eine v.a. aus (indirekter) Kostensicht relevante Altersgruppe repräsentiert jenen Bevölkerungsanteil, der als erwerbsfähig angesehen wird und in der Bundesrepublik Deutschland durch das Alterintervall von 15-65 Jahren beschrieben wird. Es sind durchaus weitere epidemiologische Sichtweisen möglich, wie z.B. der Zusammenhang von sozialer Schicht bzw. Bildungsniveau und adipösem Bevölkerungsanteil, der in der Gesundheitsberichtserstattung ebenfalls Berücksichtigung findet, für die hier vorliegende Fragestellung allerdings von untergeordneter Bedeutung ist. ${ }^{28}$

Eine gesonderte Betrachtung ist im Falle der Epidemiologie von Kindern und Jugendlichen (unter 20 Jahre) angebracht, die in Deutschland um die 16,4 Mio. der Gesamtbevölkerungszahl ausmachen, was einem relativen Anteil von rund $20 \%$ entspricht. $^{29}$ Mit der sog. KIGGS-Studie des Robert-Koch-Instituts wurde kürzlich eine umfassende Erhebung zur Kinder- und Jugendgesundheit durchgeführt und dabei neben anderen Themen ein Schwerpunkt auf Ernährung, Essstörungen und Adipositas gelegt. ${ }^{30}$ Zwar ist das Identifikationsmerkmal für Adipositas bei Kindern und Jugendlichen ebenfalls der BMI, doch ist dieser im Zusammenhang mit den alters- und geschlechtsspezifischen Eigenschaften in Verbindung zu bringen. So gelten bei Altersgruppen unter 18 Jahren populationsspezifische BMI-Perzentilen, die Übergewicht ab der 90. und Adipositas ab der 97.

\footnotetext{
${ }^{28}$ Vgl. Abbildung 2.6.2 und diesbezügliche Erläuterungen in Gesundheitsberichterstattung des Bundes, Übergewicht und Adipositas, Kapitel 2.6.1. Web-Version: http://www.gbe-bund.de/gbe10

${ }^{29} \mathrm{Vgl}$. den entsprechenden Werteabruf unter https://www-genesis.destatis.de.

${ }^{30} \mathrm{Vgl}$. http://www.kiggs.de/studie.
} 
Perzentile definieren. Ohne auf die weiteren zahlreichen methodischen Besonderheiten der Erhebungen einzugehen, stellt sich der Anteil adipöser Kinder und Jugendlicher (ab 3 Jahren) wie folgt dar: ${ }^{31}$

Abbildung 1: Übergewicht und Adipositas im Kindes- und Jugendalter in Deutschland (2007)

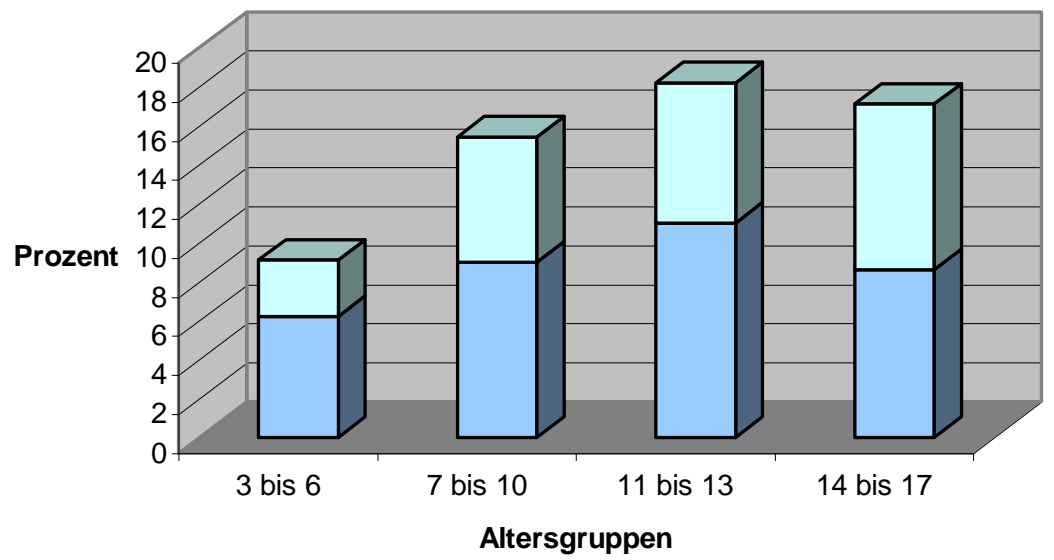

Quelle:

Eigene Darstellung auf Grundlage der Ergebnisse der KIGGS-Studie, Kurth, B. M./ Schaffrath Rosario, A. (2007): Die Verbreitung von Übergewicht und Adipositas bei Kindern und Jugendlichen in Deutschland, Tabelle 1.

Eine unterschiedliche Betrachtung von Jungen und Mädchen ist nicht erforderlich, da keine signifikanten Unterschiede zwischen den Geschlechtern auszumachen sind. Insgesamt sind von allen betrachteten 3-17 Jährigen 8,7\% übergewichtig und immerhin bereits $6,3 \%$ adipös. Der Ausprägungsschwerpunkt ist in der Altersgruppe der 11 bis 13-jährigen zu finden. Mit einer früheren Inzidenz von Adipositas besteht in der Konsequenz die höhere Wahrscheinlichkeit, dass auch Kosten und Folgekosten früher und über einen längeren Zeitraum anfallen, v.a. in Verbindung mit dem früheren Risiko, assoziierte Folge- und Begleiterkrankungen zu entwickeln. Insofern sind hier v.a. latent vorhandene Kosten verborgen, die ggf. verstärkt in der Zukunft anfallen.

\footnotetext{
${ }^{31}$ Vgl. ausführliche Hintergründe und Ergebnisse Kurth, B. M./ Schaffrath Rosario, A. (2007). Die Ergebnisse wurden im Rahmen der KIGGS-Studie ermittelt.
} 


\subsubsection{Vergleich zu den USA und Großbritannien}

Da das Problemfeld des Übergewichts und der Adipositas in der Bevölkerung neben der Bundesrepublik Deutschland alle anderen westlichen Industrienationen ebenfalls betrifft, ist ein zumindest selektiver, internationaler Vergleich durchaus angebracht, um die Situation in Deutschland unter relativen Gesichtspunkten einzuschätzen und bewerten zu können. Die Auswahl der Vergleichsländer USA und Großbritannien wurde nach Kriterien vorgenommen, die kurz und überblicksweise erläutert werden. Epidemiologische Daten von Kindern und Jugendlichen werden, v.a. mit Blick auf die im vorangegangenen Abschnitt benannten methodischen Besonderheiten bei deren Erhebung, gesondert betrachtet.

Die USA sind die Nation mit den höchsten Gesundheitsausgaben aller OECDLänder und haben zugleich den mit rund 32\% (2004) größten relativen Anteil an adipösen erwachsenen Personen. Bei einer Bevölkerungszahl von über 303 Mio. sind dies knapp 97 Mio. Menschen. Diese Rate hat sich zudem in den letzten 20 Jahren (bis 2004) mehr als verdoppelt. ${ }^{32}$ Darüber hinaus werden in den Vereinigten Staaten, wie bereits erwähnt, elementare und umfassende epidemiologische Studien angestellt, um diese für das Gesundheitswesen kritischen Entwicklungen nachzuweisen und zu beziffern. ${ }^{33}$ Insofern repräsentieren die USA zum einen jene Nation, die geradezu traditionell den größten Bevölkerungsanteil aufweist, der von Adipositas („obesity“) betroffen ist, zum anderen liefern sie eine breite Basis an Erhebungen, die von hohem Stellenwert für alle anderen Nationen sind, die zunehmend mit dieser Art Gesundheitsproblem zu kämpfen haben. Die epidemiologische Ausgangsbasis der erwachsenen Bevölkerung über 20 Jahre ist nachfolgend aufgeführt:

\footnotetext{
32 OECD Health Data (2007): How Does the United Kingdom Compare; http://www.oecd.org/dataoecd/46/4/38980557.pdf

${ }^{33}$ Hier spielen die in Tabelle 4 dieser Arbeit genannten Erkrankungen eine entscheidende Rolle.
} 
Tabelle 6: $\quad$ Prozentuale Verteilung von Übergewicht und Adipositas in den USA (2001-2004) nach Altersgruppen und Geschlecht.

\begin{tabular}{|l|r|r|r|r|}
\hline $\begin{array}{l}\text { Altersgruppen } \\
\text { In Intervallen }\end{array}$ & $\begin{array}{c}|c| \\
\text { Übergewicht } \\
\text { [BMI 25-29,9] }\end{array}$ & $\begin{array}{c}\text { Adipositas } \\
{[\mathrm{BMI} \geq 30]^{*}}\end{array}$ & $\begin{array}{r}\text { Übergewicht } \\
{[\mathrm{BMI} \text { 25-29,9] }}\end{array}$ & $\begin{array}{r}\text { Adipositas } \\
{[\mathrm{BMI} \geq 30]^{*}}\end{array}$ \\
\hline $20-34$ & 35,8 & 23,2 & 23,0 & 28,6 \\
\hline $35-44$ & 39,1 & 33,8 & 26,8 & 33,3 \\
\hline $45-54$ & 46,7 & 31,8 & 29,4 & 38,0 \\
\hline $55-64$ & 41,3 & 36,0 & 30,9 & 39,0 \\
\hline $65-74$ & 46,0 & 32,1 & 33,6 & 37,9 \\
\hline Ab 75+ & 46,9 & 19,9 & 40,5 & 23,2 \\
\hline
\end{tabular}

*Diese neuere Berechnung zieht für die Festlegung von Adipositas (obesity) einen $\mathrm{BMI}$ von $\geq 30$ heran, im Gegensatz zu dem früher üblichen US-Grenzwert von $\geq 29$ (vgl. Tabelle 2 dieser Arbeit). Die Gruppe der unter 20-Jährigen wird nicht explizit erfasst (vgl. Ergebnisteil).

Quelle: $\quad$ Teilübernahme und Teillberechnung aus Health, United States, 2007. Table 74 (1,2), S. 288-289.

Mit dem in das U.S. Department of Health \& Human Services eingebundene National Center for Health Statistics (NCHS) steht eine der zentralen epidemiologischen Datenquellen für Gesundheitsinformation und Statistik zur Verfügung, die Berechnungen zahlreicher administrativer Stellen und privater Einrichtungen einbezieht und ein umfassendes Datenwerk repräsentiert. ${ }^{34}$

Die grundlegende Systematik der Erfassung und Dokumentation von epidemiologischen Daten ist mit der in Deutschland vergleichbar, ebenso wie die Prinzipien zur Berechnung von den für die Krankheitskostenrechnung relevanten „direkten“ und „indirekten“ Kosten. ${ }^{35}$ Das relevante Altersintervall für den erwerbsfähigen Bevölkerungsanteil, welcher als Berechnungsgrundlage für indirekte Kosten erforderlich ist, kann je nach Fragestellung/ Datenverfügbarkeit und Studie uneinheitlich sein. Dennoch zeigt sich beispielhaft in Erhebungen des Departments of Health and Human Services im Bezug auf chronische Erkrankungen, dass wie

\footnotetext{
${ }^{34}$ Umfangreiche Datenquellen sind u.a. verfügbar unter http://www.cdc.gov/nchs/nhis.htm

${ }^{35}$ Eine genaue Definition und Beschreibung von direkten bzw. indirekten Kosten wird in Abschnitt 2.2 dieser Arbeit vorgenommen.
} 
auch in Deutschland der Bereich von 15 bis 65 Jahren als relevanter Erwerbs-

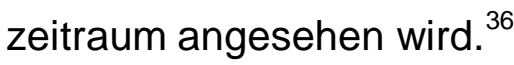

Die Altersgruppe der unter 20-jährigen Jugendlichen und Kinder wird in den USA unter anderen Gesichtspunkten als in Deutschland erfasst. Kinder und Jugendliche werden im Zusammenhang mit der altersspezifischen BMI-Bemessung ab der 95sten Perzentile als übergewichtig („overweight“) eingestuft. Die Kategorie Adipositas („obesity“) wird in den USA für Kinder und Heranwachsende nicht vergeben: ${ }^{37}$

Abbildung 2: $\quad$ Übergewicht im Kindes- und Jugendalter in den USA

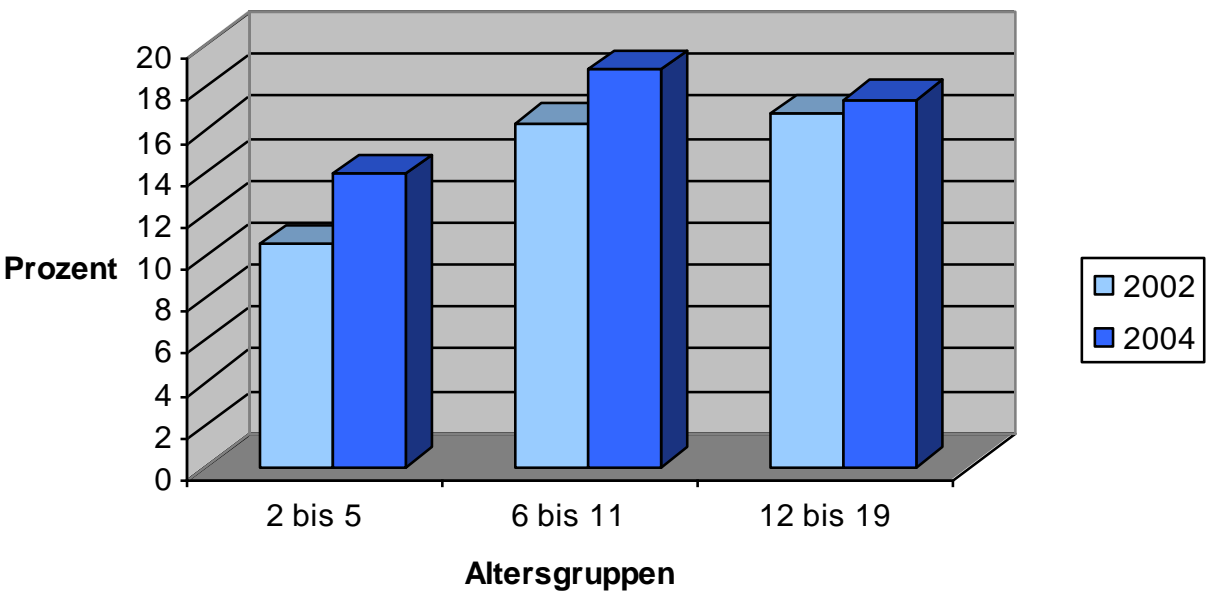

Quelle: $\quad$ Eigene Darstellung auf Grundlage der relevanten Daten aus Figure 13. Overweight and obesity, by age: United States, 1960-2004, in Health, United States, 2007, S. 104.

Die Abbildung zeigt zum einen eine prozentuale Zunahme an übergewichtigen Kindern und Jugendlichen in den USA im Vergleich der Jahre 2002 und 2004, zum anderen einen Schwerpunkt im Altersintervall der 6- bis 19-jährigen, mit

\footnotetext{
${ }^{36} \mathrm{http}: / /$ www.cdc.gov/nccdphp/overview.htm

${ }^{37}$ So wird in Health, United States, 2007 wie folgt zitiert: „For children and adolescents: overweight is defined as a body mass index (BMI) at or above the sex- and age-specific 95th percentile BMI cut points from the 2000 CDC Growth Charts: United States, obese is not defined for children." Vgl. http://www.cdc.gov/nchs/data/hus/hus07.pdf\#tocappii unter den dortigen Notes der Figure 13, S. 104.
} 
Tendenz der Zunahme von Übergewicht bei den 6 bis 11-jährigen Kindern. Die Kostenberechnungen, die im Zusammenhang mit Adipositas durchgeführt werden, berücksichtigen mit Blick auf die direkten Kosten alle Altersgruppen also auch den Bevölkerungsanteil unter 20 Jahren unter o.g. Bedingungen. indirekte Kosten beziehen sich auf die erwerbstätige Bevölkerung und schließen somit Kinder unter 15 Jahren aus. (vgl. Ergebnisteil).

In Großbritannien liegt der relative Anteil von adipösen Erwachsenen mit rd. 26\% (2006) zwar niedriger als in den USA, aber dafür wurde dort in den vergangenen 20 Jahren (bezogen auf 2005) ein Anstieg von Betroffenen um mehr als das Dreifache beobachtet. Bei einer Einwohnerzahl von rund 60,6 Mio. sind dies ca. 15,7 Mio. Betroffene.$^{38}$ England und Wales weisen mit 53,7 Mio. den größten Einwohneranteil auf und bilden somit auch den Schwerpunkt der Betrachtungen.

Tabelle 7: $\quad$ Prozentuale Verteilung von Übergewicht und Adipositas in Großbritannien (hier nur England/ Wales) (2006) nach Altersgruppen und Geschlecht (gerundet).

\begin{tabular}{|l|r|r|r|r|}
\hline $\begin{array}{l}\text { Altersgruppen } \\
\text { In Intervallen }\end{array}$ & $\begin{array}{r}|c| \\
\text { Uänner [\%] }\end{array}$ & \multicolumn{2}{|c|}{ Frauen [\%] } \\
\hline [BMI 25-29,9] & $\begin{array}{l}\text { Adipositas } \\
{[\text { [BMI } \geq 30]^{*}}\end{array}$ & $\begin{array}{l}\text { Übergewicht } \\
\text { [BMI 25-29,9] }\end{array}$ & $\begin{array}{l}\text { Adipositas } \\
{[\mathrm{BMI} \geq 30]^{*}}\end{array}$ \\
\hline $16-24$ & 24,0 & 10,0 & 19,0 & 13,0 \\
\hline $25-34$ & 40,0 & 22,0 & 27,0 & 20,0 \\
\hline $35-44$ & 46,0 & 27,0 & 27,0 & 27,0 \\
\hline $45-54$ & 46,0 & 30,0 & 32,0 & 30,0 \\
\hline $55-64$ & 44,0 & 36,0 & 33,0 & 33,0 \\
\hline $65-74$ & 48,0 & 32,0 & 33,0 & 39,0 \\
\hline Ab 75+ & 51,0 & 18,0 & 40,0 & 29,0 \\
\hline
\end{tabular}

*Die Gruppe der unter 16-Jährigen wird hier nicht explizit erfasst.

Quelle: $\quad$ Teilübernahme un Teilberechnung aus Health Survey for England (2006) Table 5.5 "Trends in Body Mass Index (BMI), underweight, overweight and obesity prevalence, 1994-2006, by age and sex", S. 102-103.

\footnotetext{
${ }^{38}$ Vgl. http://www.statistics.gov.uk/downloads/theme health/HSQ37.pdf, Table 1.4 Population by age and sex, S. 31. (Bevölkerungshochrechnungen).
} 
Die offizielle Gesundheitsstatistik wird über den Nationalen Gesundheitsdienst (National Health Service NHS) und das 1999 gegründete National Institute for Health and Clinical Excellence (NICE) bereitgestellt. Aber auch weitere Quellen liefern epidemiologische Beiträge zu dem Themenbereich der Adipositas. ${ }^{39}$ Die Schätzungen des Bevölkerungsanteils an Kindern und Jugendlichen unter 20 Jahren belaufen sich auf rd. 15 Mio. Aktuelle Veröffentlichungen der „National Statistics" zum Ernährungszustand dieser Altersgruppe liegen lediglich für das Jahr 2000 vor, diesbezüglich neuere Erhebungen waren nicht zu ermitteln.

Abbildung 3: Relation zwischen Übergewicht und Adipositas bei Kindern und Heranwachsenden in Großbritannien für das Jahr 2000, nach Geschlecht.

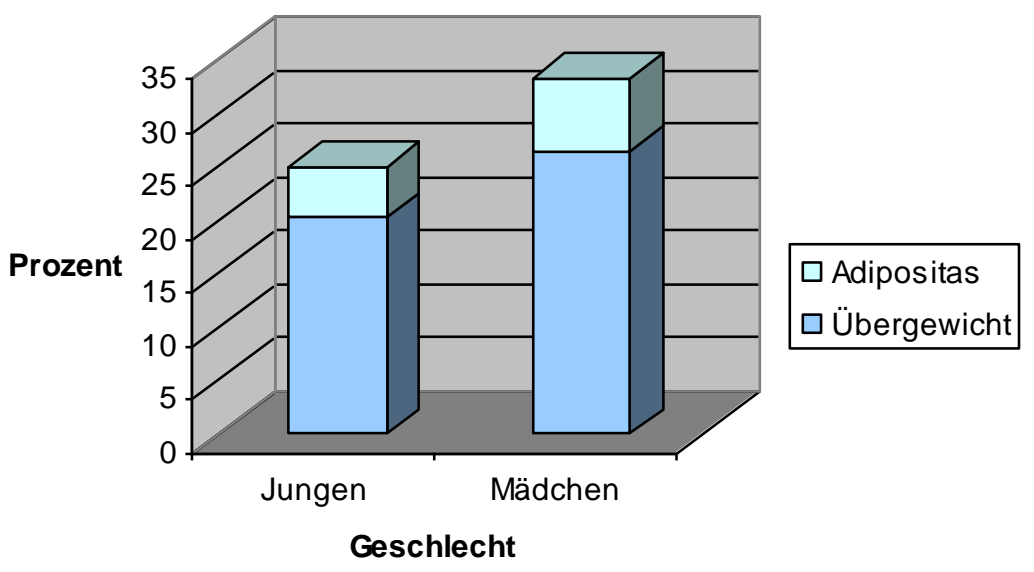

Quelle: $\quad$ Eigene Zusammenstellung und Darstellung auf Grundlage des National Statistics Berichts „Diet \& Nutrition Proportion of overweight children up“, veröffentlicht unter http://www.statistics.gov.uk/cci/nugget.asp?id=718.

Es zeigt sich hierbei, dass britische Mädchen mit insgesamt rund 33\% deutlich stärker betroffen sind als Jungen mit rund 25\%. Da zwischen den Ländern teils unterschiedliche Definitionen und Erhebungsmethoden v.a. mit Bezug auf die Prävalenzen angewendet werden, sind vergleichende Aussagen nur bedingt möglich bzw. sinnvoll. Dennoch zeigt sich im europäischen Vergleich, dass in Großbritannien, wie auch in Deutschland, der Anteil der Bevölkerung (insgesamt)

\footnotetext{
${ }^{39}$ Vgl. z.B. ERPHO Eastern Region Public Health Obeservatory - Improving access to population health data, methods and expertise. http://www.erpho.org.uk/topics/Obesity/.
} 
mit Übergewicht und Adipositas deutlich höher ist als in anderen EU-Staaten ${ }^{40}$. Insofern ist davon auszugehen, dass Großbritannien als ein für die hier zu behandelnde Fragestellung - sollten auch aussagekräftige Kostenanalysen zur Verfügung stehen - besonders geeignetes Vergleichsland aufzufassen ist, auch wenn z.B. aufgrund weiterer Zusammenhänge oder Gesichtspunkte sicherlich auch ein Vergleich mit anderen EU-Nationen oder Nationen der europäischen Region gemäß WHO-Definition ${ }^{41}$ möglich gewesen wäre. ${ }^{42}$

Zusammenfassend stellen sich die Daten zu Übergewicht und Adipositas beider Bezugsländer im Vergleich zu Deutschland wie folgt dar:

Abbildung 4: Vergleich von Übergewicht und Adipositas zwischen Deutschland, USA und Großbritannien (Erwachsene > 20 Jahre)

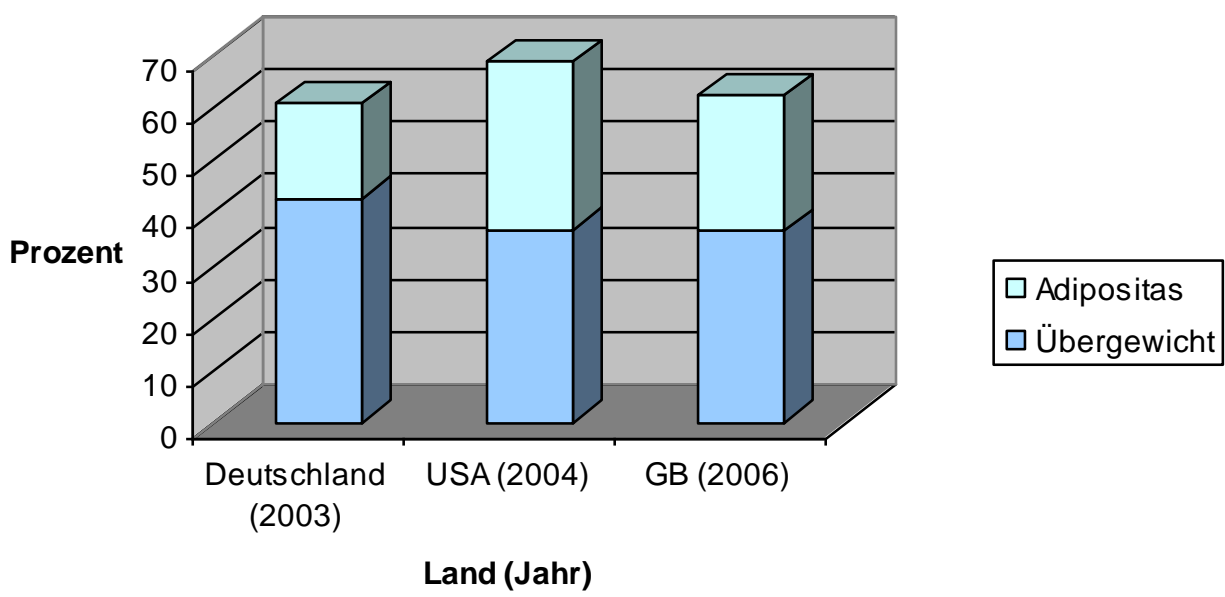

Quelle: $\quad$ Eigene Zusammenstellung und Darstellung auf Grundlage der Ergebnisse des gesamten Abschnitts 1.4 „Epidemiologische Datenbasis“.

Als abschließendes Argument für die Auswahl von Großbritannien wie auch den USA als Vergleichsnationen spricht zudem, dass die Bevölkerungsdaten zur

\footnotetext{
${ }^{40} \mathrm{Vgl}$. Gesundheitsberichterstattung des Bundes, Übergewicht und Adipositas, Kapitel 2.6.1 in Verbindung mit dortiger Quellenangabe Eurostat (2003) Health in Europe: results from 1997 to 2000 surveys. European Communities.

${ }^{41}$ Vgl. hierzu WHO (2005) und auch Fußnote 147 dieser Arbeit.
} 
Ermittlung von Adipositas als tragfähiger anzusehen sind als die anderer Länder. Dies ist darauf zurückzuführen, dass die Angaben auf objektiven BMI-Messungen über Körpergröße und Gewicht basieren, während die Bestimmung dieser Größen in den anderen EU-Vergleichsländern verstärkt auf Schätzungen und Selbstauskünften beruhen, bei denen die tatsächlich bestehende Prävalenz von Adipositas in der Regel eher zu gering bewertet wird.

\subsection{Konkrete Fragestellung}

Adipositas wird im medizinischen Kontext v.a. aus Sicht der Entstehungsursachen und Behandlungsmöglichkeiten betrachtet. Hierzu existiert eine Reihe von Beiträgen, die Zusammenhänge von körperlicher Konstitution, Prädisposition, Ernährung, Bewegung, Risikopotenzial und damit verbundene Stoffwechselaspekte berücksichtigen. Auch die bestehenden epidemiologisch-soziologischen Gründe zunehmender gesellschaftlicher Probleme von Fettleibigkeit in modernen Industrienationen werden untersucht. Ein erkanntes, nach wie vor bestehendes Defizit allerdings ist die konkrete Verknüpfung dieser Aspekte mit ökonomischen Sichtweisen, die in der hier vorliegenden Arbeit im Mittelpunkt steht. Eine hohe Kostenbelastung durch Adipositas wird zwar durchgängig als wachsendes gesellschaftliches und v.a. gesundheitspolitisches Problem angesehen, das in besonderem Maße auch Deutschland betrifft. Die explizite Bezifferung bzw. möglichst genaue Schätzung von diesbezüglichen Gesamtkosten wurde in der Vergangenheit wie auch aktuell allerdings kaum vorgenommen. Hauptgründe, die sich auch für die nachfolgenden Berechnungen gezeigt haben, werden u.a. darin gesehen, dass:

- statistische Datenquellen nur bedingt vorhanden bzw. zugänglich sind

- Datengrundlagen teilweise uneinheitliche Inhalte repräsentieren und nicht durchgängig aktuell sind

- die erforderlichen Berechnungen restriktive Annahmen erfordern und dabei dennoch einen hohen Komplexitätsgrad aufweisen

- keine durchgängig einheitlichen Berechnungsregelungen bestehen und Vergleiche sowie Validierungen nicht unbedenklich sind

\footnotetext{
${ }^{42} \mathrm{Vgl}$. den weiteren Ländervergleich im Rahmen der Gesundheitsberichterstattung des Bundes sowie WHO (2003) Der europäische Gesundheitsbericht 2002. In: WHO Regionale Veröffentlichungen (Hrsg.) Europäische Schriftenreihe Nr. 97.
} 
- vorgenommene Berechnungen je nach Standpunkt kritisch zu betrachten sind und ggf. gesundheitspolitische Weichenstellungen nach sich ziehen, die weitreichende finanzielle Konsequenzen und erforderlichen Handlungsbedarf aufzeigen können

Mit der konkreten Fragestellung zur Berechnung von Gesamtkosten der Adipositas in Deutschland bewegt man sich somit in einem Themengebiet, welches mit zahlreichen Unsicherheiten und fraglos kritischen Aspekten behaftet ist. Mit Hilfe von Annahmen, Vergleichs- und Hochrechnungen soll der Gegenstand der Adipositas dennoch um diese medizinisch-ökonomische Komponente erweitert werden und am Ende nachvollziehbare und diskutierbare Ergebnisse liefern. 


\section{Material und Methodik}

\subsection{Identifikation der Krankheitskosten}

Die Bestimmung und Erfassung bzw. Berechnung von Krankheitskosten ist für alle diesbezüglich zu erstellenden Untersuchungen eine komplexe Anforderung, der grundsätzlich unterschiedliche Ermittlungsansätze und -auffassungen zugrunde liegen können. Rein sachlich-ökonomisch betrachtet bewertet der Begriff der Kosten die Nutzung, den Verbrauch bzw. den nachhaltigen Verlust von zur Verfügung stehenden Ressourcen. ${ }^{43}$ Die Ressourcen im Rahmen der Betrachtung des Gesundheitssektors sind demnach diejenigen Inputfaktoren - Arbeit und Kapital -, die für die Gesundheit von Einzelnen bzw. in Addition für eine betrachtete Gesellschaft und/ oder definierte Region eingesetzt werden. Mit der zusätzlichen Anforderung, eine Kostenanalyse lediglich für ein bestimmtes medizinisches Segment - wie hier die Kosten in Zusammenhang mit der Adipositas - vorzunehmen und dabei eine nationale Perspektive - wie hier die Bundesrepublik Deutschland abzubilden, erfordert weitergehende Überlegungen und Methoden.

\subsubsection{Ermittlung der Kostendaten}

Die Bestimmung der erforderlichen Daten basiert auf unterschiedlichen Quellen und Berechnungen. Zur Berechnung von aggregierten Kosten bzw. Gesamtkosten bezogen auf die gesamte Bevölkerung kann zum einen auf die offizielle Gesundheitsausgaben- und Krankheitskostenrechnung des Statistischen Bundesamtes zurückgegriffen werden. ${ }^{44}$ Darin werden Gesundheitsausgaben eines jeweiligen Bezugsjahres für erfasste Leistungen aller ambulanten, teilstationären und stationären Einrichtungen dokumentiert. Darüber hinaus finden die Ausgaben des Gesundheitsschutzes, der Verwaltung, sonstiger Einrichtungen sowie privater Haushalte Eingang in die Statistik. Zudem steht eine methodisch als zentral anzusehende Studie des Bundesgesundheitsministeriums (BMG) aus dem Jahr 1993 zur Verfügung, die sich auf „ernährungsabhängige Krankheiten und ihre

\footnotetext{
${ }^{43}$ Vgl. Henke, K.-D./ Behrens C. et. al. (1986). S. 14 sowie Schneider, H./ Schmidt, A. (2004), S. 3.

${ }^{44}$ Statistisches Bundesamt, Statistisches Jahrbuch 2007, Abschnitt 9 Gesundheitswesen.
} 
Kosten“ bezieht. ${ }^{45}$ Gemäß der „Evidenzbasierten Leitlinie zu Prävention und Therapie der Adipositas" aus dem Jahr 2007 wird allerdings auf die systematische Unterschätzung der Kosten für Adipositas durch die Erhebung des BMG hingewiesen, da zum einen Komorbiditäten von Adipositas keine Berücksichtigung fänden sowie die Diagnose „Adipositas" nur selten gestellt und somit erfasst würde. ${ }^{46} \mathrm{Im}$ Rahmen der nationalen Datenerfassung/ Informationsbereitstellung stehen des Weiteren Institute, Krankenkassen (v.a. AOK), Deutsche Rentenversicherung Bund, Pharmahersteller sowie die jeweiligen Gesellschaften ${ }^{47}$ für bestimmte Erkrankungen oder Syndrome im Vordergrund.

Die Berechnungen der Gesamtergebnisse für ein Jahr basieren teilweise auf Ergebnissen zeitlich möglichst benachbarter Jahre, denn aufgrund der unterrepräsentierten Datensituation sind nicht alle Kosten jeweils für dasselbe Jahr abschätzbar. Die Gesamtergebnisse, die als Berechnungen für ein Bezugsjahr ausgewiesen werden, stellen demnach Mischberechnungen aus mehreren Betrachtungszeiträumen dar (Schwerpunkt 2003), die zu einer Gesamtkostensicht hochgerechnet werden. Eine zentrale Rolle spielen dabei Erhebungen im Rahmen von nationalen und internationalen Studien und Auswertungen, insbesondere zu den einzelnen Folge- und Begleiterkrankungen von Adipositas, die aus den einschlägigen Fach-/ Zeitschriftendatenbanken recherchiert werden. Weitere Erhebungen, wie z.B. die Ermittlung von Ernährungsberatungskosten im ambulanten Behandlungsfeld oder genaue Zahlen zu durchgeführten Operationen der Adipositas im stationären Bereich, werden zwar benötigt, jedoch liegen hierzu kaum repräsentative Daten vor. Daher muss in solchen Fällen auf die Erkenntnisse von teilweise nur sehr spärlich vorhandenen Auswertungen zurückgegriffen werden, wodurch gleichzeitig ein zentrales Informationsdefizit aufgezeigt wird. Auf Grundlage aller repräsentativen Quellen werden eigene Berechnungen und Vergleiche durchgeführt, die sich an etablierten Methoden und Vorgaben, z.B. zur Berechnung beizumessender Kostenanteile assoziierter Erkrankungen, orientieren und an den jeweiligen Stellen genauer beschrieben werden.

\footnotetext{
${ }^{45}$ Kohlmeier, L. et. al (1993).

${ }^{46} \mathrm{Vgl}$. Hauner, H. et al. (2007), S. 51.
} 


\subsubsection{Schätzung von Kosten}

Es gilt, kurz die Hintergründe dafür aufzuzeigen, warum nicht alle einzelnen Kostenanteile, die in der Analyse zu einem aggregierten Gesamtkostenbild führen sollen, explizit in monetärer Form vorliegen und geschätzt werden müssen. ${ }^{48}$ Dabei sind v.a. drei wesentliche Aspekte zu unterscheiden:

1) Teile der Daten fließen als Hochrechnungen in die Analyse ein. Die Gründe hierfür können z.B. sein, dass die zu ermittelnden Daten nicht in erforderlichem Umfang oder Zeitraum vorliegen bzw. im Rahmen einer Studie oder Auswertung nur für Stichproben erhoben werden konnten, die im Zuge dessen hochgerechnet/ extrapoliert werden müssen. Dazu wird notwendigerweise zum Teil auf Daten aus dem Ausland zurückgegriffen oder sie werden aus Befragungen/ Teilberechnungen oder Berechnungen in vergleichbaren Zusammenhängen herangezogen.

2) Ein anderer Teil der ermittelten Daten wird im Rahmen bestimmter Fragestellungen mit Relationen gewichtet, die in vergleichbaren Untersuchungszusammenhängen ermittelt wurden und für Teile der Kostenberechnungen heranzuziehen sind. Dies wird dann erforderlich, wenn einer Analyseanforderung entsprochen werden muss, wie in dem vorliegenden Fall z.B. die Berechung von Kosten für Begleit- und Folgeerkrankungen von Adipositas unter einer relativen Unsicherheit. Hierzu werden auf der Grundlage der nach Möglichkeit empirisch-langfristig nachgewiesenen medizinischen Zusammenhänge und statistischen Wahrscheinlichkeiten bestimmte beizumessende Anteile von Kosten abgeschätzt, um assoziierte Krankheiten als Kostendeterminanten für Adipositas verursachungsgerecht mit zu berücksichtigen.

3) Schließlich besteht für den in Kostenanalysen ebenfalls häufig durchgeführten prognostischen Bereich zwingend die Anforderung, Kostenschät-

\footnotetext{
${ }^{47}$ Im Falle der Adipositas ist beispielhaft der Deutsche Adipositas-Gesellschaft e.V. zu nennen.

${ }^{48}$ Vgl. Hauner, H. et al. (2007), a.a.O., S. 50 ff.
} 
zungen vorzunehmen. Hierzu werden Annahmen herangezogen, welche zu unterschiedlichen Szenarien führen können. Aufgrund der geforderten Zukunftssicht und der damit verbundenen absoluten Unsicherheit, bestehen keinerlei Alternativen zu Kostenschätzungen, was somit auch keiner weiteren Erläuterung bedarf.

Alle drei Ebenen, auf denen Kostenschätzungen stattfinden, fließen in die Analyse ein. Während die erste Art der Schätzung dazu dient, möglichst eine Vollständigkeit aller explizit erfassbaren Diagnosen und im Gesundheitsmarkt tatsächlich entstandenen monetären Größen (z.B. direkte Kosten für Medikamente) herbeizuführen, wird durch die unter Punkt 2) beschriebene Art der Kostenschätzung eine erforderliche Zusatzrechnung auf bestehenden oder ermittelten Daten vorgenommen. Sie hat die Zielsetzung, aus den bereits vorliegenden Kosten und weiteren Daten (z.B. Ausfallzeiten) die Berechnung jener Kostenanteile vorzunehmen, die dem bestimmten medizinischen Gegenstand Adipositas beigemessen werden und über keine offizielle Statistik zur Verfügung stehen.

Unter den Gegebenheiten der gängigen Datenerhebungssystematik und der Studienlage ist die Schätzung eines Teils der Kosten somit unumgänglich und aus dem Anspruch einer Gesamtkostenbetrachtung heraus gleichzeitig unverzichtbar. Dies scheint mit Blick auf die erwarteten Ergebnisse einer möglichst voll umfassenden Kostenberechnung dieses Teilbereichs des Gesundheitswesens in jedem Fall dann akzeptabel zu sein, wenn die ganzheitliche Sicht der Daten bzw. die Datenerhebung weder einer subjektiven Auffassung einer Regierung, der Leistungserbringer oder Kostenträger, noch sonstigen anderen Interessengruppen untergeordnet wird und eine Neutralität der Daten soweit als möglich gegeben ist.

\subsubsection{Zusätzliche Kostenberechnungen}

Von besonderem Interesse für die Analyse ist die Berechnung der Kostenanteile von assoziierten Erkrankungen der Adipositas, die für einen wesentlichen Teil der Arbeit erforderlich sind. Es wird sich zeigen, dass zu deren Ermittlung eine Risikoschätzung mit Hilfe angenommener Wahrscheinlichkeiten für Begleit- und Folgeerkrankungen aufgrund von Adipositas notwendig sein wird, die sich über den 
sog. „Bevölkerungsbezogenen beizumessenden Anteil“ vornehmen lässt. Diese im amerikanischen Sprachgebrauch als "PAF“ (population attributable fraction) bezeichnete Größe geht auf eine anteilige Bestimmung des relativen Risikos (RR) für Folgeerkrankungen aufgrund der Adipositas zurück. ${ }^{49}$ Hierzu werden die ermittelten direkten und indirekten Kosten (der nach ICD 10) eigenständig geführten Erkrankungen herangezogen und daraus ein sog. risikobewerteter Anteil bestimmt. Durch dieses Vorgehen wird es möglich, dass - gemäß einem Verursachungsprinzip - die Kosten der (relevanten) Begleit- und Folgeerkrankungen desaggregiert und der Adipositas zugewiesen werden können. Zu diesem Zweck wird die Berechnungsformel der beizumessenden Anteile in anerkannten Studien im Allgemeinen wie folgt beschrieben: ${ }^{50}$

$\mathrm{PAF}=\mathrm{P}(\mathrm{RR}-1) /[\mathrm{P}(\mathrm{RR}-1)+1] \quad$ „Attributable Fraktion der Bevölkerung“.

Die PAF kann somit kurz als der Prävalenzanteil einer Erkrankung bezeichnet werden, der aufgrund einer bestimmten Exposition, z.B. Adipositas, verursacht wird. Die Fraktion drückt demnach anders herum formuliert jenen Anteil von Erkrankungsfällen aus, der durch Wegfall des Risikos vermieden würde. Demgemäß entspricht $P$ der Wahrscheinlichkeit, dass eine Person innerhalb einer betrachteten Bevölkerungsgruppe (z.B. Deutsche) an Adipositas leidet. RR bezeichnet das Relative Risiko für die Existenz einer adipositas-assoziierten Erkrankung der mit Adipositas exponierten Personen.

Grundsätzlich wird mit allen diesbezüglichen Ansätzen das Ziel verfolgt, möglichst aussagekräftige Anhaltspunkte darüber zu erhalten, wie groß der Einfluss von Adipositas (bzw. anderen Expositionen) auf die Entstehung und Persistenz von mit Adipositas assoziierten Erkrankungen ist. Mit der Wahl dieses Ansatzes muss man gleichzeitig die zu erwartenden Konsequenzen für die Kostenbetrachtung anführen. Einerseits wird durch die Berechnungsmöglichkeit mit Hilfe von identifizierten bevölkerungsbezogenen, beizumessenden Anteilen eine bestenfalls

\footnotetext{
${ }^{49}$ Vgl. Wolf, A.M./ Colditz, G.A. (1994), S. 34ff.

${ }^{50}$ Vgl. z.B. Birmingham C. L. et.al. (1999), S. 483-488 sowie Rockhill B, Newman B, Weinberg C. (1998), S. 15-19.
} 
"gerechtere" und „realitätsnähere" Kostenbewertung und -zuordnung angestrebt. In der Summenbetrachtung ändern sich dabei die Gesundheitskosten nicht. Stehen jedoch, wie in dieser Arbeit, speziell die Kosten für einen bestimmten medizinischen Gegenstand - wie hier die Adipositas - im Mittelpunkt der Betrachtung, kann eine rechnerische Umschichtung von Kosten und damit eine Verschiebung von Kostenschwerpunkten, durchaus weitreichende gesundheitspolitische und -ökonomische Folgen nach sich ziehen. Andererseits darf nicht übersehen werden, dass neben der Adipositas auch andere Dispositionen oder (Primär-) Erkrankungen mit der Entstehung von den gleichen Folgeerkrankungen in Verbindung gebracht werden und sich durchaus mehrere Einflussfaktoren auf eine Krankheitsentstehung oder einen -verlauf auswirken können, denkt man z.B. an genetische Zusammenhänge oder die Existenz von zeitgleich bestehenden Risiken, beispielsweise durch Rauchen oder Alkohol. Diese Erkenntnis führt zwar nicht dazu, den Ansatz von PAF in Frage zu stellen oder gar zu verwerfen. Vielmehr soll verdeutlicht werden, dass unter Berücksichtigung genannter Zusammenhänge, die Anwendung des distributiven Vorgehens auch unter kritischen Aspekten gesehen wird und hier keinesfalls eine Berechnungsmethode verwendet wird, die von jeglichen Schwachstellen freizusprechen ist.

\subsection{Klassifizierung der Kosten}

Der Erfassung und Klassifizierung von (zunächst unspezifischen) Gesundheitskosten liegen zahlreiche bereits seit den 60er Jahren existierende methodische Überlegungen aus Deutschland, v.a. und verstärkt aber aus der amerikanischen Gesundheitsforschung zugrunde. ${ }^{51}$ Die Gliederung der erforderlichen Kosten zur Bewertung von Adipositas basiert auf der zentralen Unterscheidung in indirekte und direkte Kosten. Während sich definitionsgemäß die indirekten Kosten mit den monetär bewerteten, messbaren Konsequenzen aus einer Erkrankung beschäftigen (z.B. bewertete Ausfallzeiten bzw. Produktivitätseinbußen aufgrund von Arbeitsunfähigkeit oder Tod) und demgemäß „indirekt“ ermittelt werden, ist die Bedeutung der direkten Gesundheitskosten durch die monetäre Erfassung all jener Kosten gegeben, die unmittelbar - also konsequenterweise „direkt" - der

\footnotetext{
${ }^{51}$ Vgl. Henke, K.-D./ Behrens C. et. al. (1986), Abschnitt B.
} 
untersuchten Erkrankung zuzuweisen sind. ${ }^{52}$ Dies sind z.B. die Kosten für Medikamente, Hilfsmittel oder Behandlungskosten (inklusive gewichteter assoziierter Erkrankungen). Sog. intangible Kosten werden aufgrund der Vollständigkeit kurz beschrieben, jedoch nicht explizit berücksichtigt. Die vorgestellte Art der Kostenklassifizierung ermöglicht die Berücksichtigung von Expertisen, die auf vergleichbarer Datengrundlage aufgebaut sind.

\subsubsection{Direkt zurechenbare Kosten}

Die hier verwendete Berechnungsweise zur Ermittlung der direkten Kosten einer Erkrankung repräsentiert einen sog. prävalenzbasierten Ansatz, mit dem für eine bestimmte Bezugsperiode (i.d.R. ein Kalenderjahr) diejenigen Kosten ermittelt werden, die durch eine adipöse Körperkonstitution - also mit einem BMI $\geq 30$ $\left[\mathrm{kg} / \mathrm{m}^{2}\right]$ - verursacht werden. Alternativ könnte der ebenfalls anerkannte inzidenzbasierte Ansatz verwendet werden, der alle direkten Kosten von neu als adipös diagnostizierten Personen/ Patienten zu berücksichtigen hätte. Dieser Ansatz wird allerdings nur selten angewendet und kommt auch hier nicht zum Tragen, da er eine Reihe schwierig bis kaum zu ermittelnde Daten/ Datenreihen erfordert, wie z.B. bzgl. der Krankheitsdauer bzw. des -verlaufs oder auch zum Gesundheitsverhalten entweder für die Restlebenszeit eines Patienten oder ggf. bis zu dessen Heilung. ${ }^{53}$

Grundsätzlich liegt allen anfallenden direkten Kosten ein tatsächlicher Ressourcenverbrauch von Gesundheitsgütern und -leistungen zugrunde. Die direkten Kosten umfassen neben Krankenhausaufenthalten und den während dieser Zeit durchgeführten Behandlungen und Therapien auch ambulante Arztbesuche, verordnete (zugelassene) Medikamente, Pflegekosten und erforderliche Rehabilitationsmaßnahmen sowie alle anderen in direktem Zusammenhang stehenden (erfassbaren oder schätzbaren) Kosten einer Erkrankung. Zentrale Kostendeterminanten im Falle der Adipositas sind die ärztliche Ernährungsberatung, die medikamentöse Behandlung mit zugelassenen Medikamenten, die Psychotherapie und die therapeutischen Eingriffe zur Gewichts- und Fettreduktion durch in

\footnotetext{
${ }^{52}$ Vgl. z.B. Greiner, W. (1999), S. $30 \mathrm{ff.}$
} 
erster Linie chirurgische Maßnahmen (v.a. Magenverkleinerung) ${ }^{54}$ Das für die Praxis relevante Problem hierbei ist, dass Adipositas im Sinne des SGB keine Krankheit repräsentiert und den Patienten i.d.R. keine Kosten erstattet werden. ${ }^{55}$ Die direkten Kosten beinhalten definitionsgemäß zusätzlich Verwaltungskosten der Leistungserbringer sowie sämtlicher öffentlicher und privater Einrichtungen, die in Deutschland Gesundheitsleistungen finanzieren. Aufgrund der Datenlage können diese Kostenanteile hier nicht gesondert berechnet werden, sondern müssen als impliziter Bestandteil der übrigen direkten Kosten angenommen werden, was zu Abweichungen v.a. gegenüber der Gesundheitskostenrechnung des Bundes führen kann. Weitere direkte Kosten entstehen durch die assoziierten Erkrankungen, die aus Übersichtlichkeitsgründen gesondert behandelt werden.

\subsubsection{Indirekt zurechenbare Kosten}

Direkte Kosten repräsentieren nur jenen Teil der Kosten, der über die Leistungsabrechnungen der definierten Einrichtungen in direkter Weise monetär ermittelbar ist. Des Weiteren stellt der potenziell erwerbstätige bzw. erwerbsfähige Bevölkerungsanteil die Berechnungsbasis für volkswirtschaftlich relevante, krankheitsbedingte Ressourcenverluste - indirekte Kosten - dar. Eine ausschließliche Berücksichtigung von Menschen im erwerbsfähigen Alter klammert alle anderen Bevölkerungsanteile (z.B. Rentner und auch Kinder unter 15 Jahren) aus, was häufig zur Kritik an diesem Vorgehen führt. ${ }^{56}$

In den etablierten Kostenanalysen haben sich jene Ansätze durchgesetzt, welche die indirekten Kostenkriterien

- Arbeitsunfähigkeit

- Invalidität

- Mortalität

anhand von durchschnittlichen Lebenszeiteinkommen bewerten, so dass auch hier diesem Vorgehen gefolgt wird. Indirekte Kosten zählen genau genommen

\footnotetext{
${ }^{53}$ Vgl. Greiner, W. (1999), S. 54.

${ }^{54}$ Vgl. Hauner et al. (2000) sowie Schneider, H/ Schmid, A. (2004), a.a.O.. S. 5 und S. $14 \mathrm{ff.}$

${ }^{55}$ Siehe hierzu auch Abschnitt 1.3.1.
} 
nicht zu den Ausgaben des Gesundheitswesens, sondern repräsentieren einen krankheitsbedingten Verlust an Wertschöpfungspotenzial einer Volkswirtschaft, gemessen durch die o.g. Faktoren. ${ }^{57}$ Nichtsdestotrotz ist ihre Erfassung dann zwingend erforderlich, wenn eine Gesamtkostenbetrachtung einer Erkrankung vorgenommen werden soll. Diese mittelbar hervorgerufenen volkswirtschaftlichen Ressourcenverluste, deren Entstehung in erster Linie den beizumessenden Anteilen der Begleit- und Folgeerkrankungen von Adipositas und nicht der Adipositas selbst zuzusprechen sind, werden in der Kostenberechnung des Statistischen Bundesamtes ausdrücklich nicht berücksichtigt. ${ }^{58}$ Dagegen werden Ausfallzeiten durch Arbeitsunfähigkeit, Invalidität und Tod in Abhängigkeit von den ICD 10 Ziffern ermittelt, wenngleich lediglich als aggregierte Größen und nicht nach Altersgruppen gegliedert, so dass andere Quellen (v.a. AOK, übrige GKV, Rentenversicherungsträger) herangezogen werden müssen. Die Erhebung der Ausfallzeiten durch Arbeitsunfähigkeit folgt einem Prävalenzansatz und berücksichtigt retrospektiv die gesamten krankheitsbedingten Fehlzeiten der erwerbstätigen Bevölkerung eines betreffenden Berichtsjahres. Der Berechnung der verlorenen Erwerbstätigkeitsjahre infolge von Invalidität und Mortalität liegt dagegen ein inzidenzbezogener Ansatz zugrunde, welcher über das aktuelle Berichtsjahr hinausgehend, den zukünftig vermuteten, irreversiblen Arbeitsausfall einbezieht. ${ }^{59}$

Zur Bestimmung des indirekten Anteils der Kosten sind bestimmte Annahmen zu treffen, welche die Rahmenbedingungen für deren Herleitung festlegen. Die Literatur führt im Wesentlichen drei verschiedene Modellansätze an: ${ }^{60}$

1) Der erste wird als sog. Humankapitalansatz bezeichnet und unterstellt, dass durch den Ausfall eines von Adipositas selbst und/ oder von einer (oder mehreren) Attributiverkrankung(en) betroffenen Patienten aus dem

\footnotetext{
${ }^{56}$ Kohlmeier, L. et. al (1993), S. $264 \mathrm{ff}$.

${ }^{57}$ Henke, K.-D. et al. (1997), S. 3. sowie www.gbe-bund.de insbesondere Krankheitskostenrechnung Methodik, Abschnitt 3.1 Abgrenzung von Krankheitskosten.

${ }^{58} \mathrm{Vgl}$. ebenda.

${ }^{59} \mathrm{Vgl}$. Kohlmeier, L. et al. (1993), S. $234 \mathrm{ff}$.
} 
Erwerbsleben, ein gesamtwirtschaftlicher bzw. gesamtgesellschaftlicher Produktivitätsverlust eintritt. Dieser Arbeitsausfall wird als Opportunitätskostengröße mit jenem monetären Produktionswert beziffert, der bis zum gesetzmäßigen Rentenbeginn hätte von den betroffenen Personen erwirtschaftet werden können. ${ }^{61}$ Eine zentrale und zugleich kritische Annahme hierbei ist, dass diese Personen mit der gleichen Wahrscheinlichkeit wie nicht erkrankte Personen erwerbstätig geblieben wären. Zudem bleiben Personen außerhalb des Erwerbsalters wie erwähnt unberücksichtigt.

2) Der sog. Friktionskostenansatz hingegen setzt die Annahme voraus, dass jeder durch Ausfall längerfristig frei gewordene Arbeitsplatz in einem absehbaren Zeitraum durch eine bis dahin arbeitslose Person ersetzt werden kann. Diese Annahme führt dazu, dass sich der (potenziell große) Produktivitätsverlust bei langer Krankheit oder frühzeitigem Tod in Wirklichkeit als relativ gering herausstellt. Auch in diesem Ansatz werden nur Personen aus der Erwerbsschicht berücksichtigt. ${ }^{62}$

3) Mit der sog. Zahlungsbereitschafts-Methode soll ein Geldbetrag ermittelt und festgelegt werden, den ein Mensch zu zahlen bereit bzw. fähig zu bezahlen wäre, um z.B. eine Lebensverlängerung zu erreichen oder auch eine Invalidität/ einen Arbeitsausfall zu vermeiden. ${ }^{63}$ Aufgrund der schwierigen Ermittlung und Problemen mit überbewerteten Präferenzen ist dieser Ansatz als kaum praktikabel anzusehen, wenngleich er von der Zielsetzung zu würdigen ist. ${ }^{64}$

Ohne tief greifender auf die beschriebenen Ansätze einzugehen, sind sie alle mehr oder weniger kritisch zu bewerten und je nach Anforderung bzgl. ihrer

60 Vgl. Welte, R. et.al. (2004), S. 3 http://www.helmholtz-muenchen.de/neu/Wir ueber uns/jahresbericht/2004/031 036 igm akt.pdf sowie Kohlmeier, L. et. al (1994), a.a.O. S. $250 \mathrm{ff}$.

${ }^{61}$ Vgl. Oberender, P./ Zerth, J. (2006), S. 537.

${ }^{62}$ Vgl. Welte, R, et. al (2004), a.a.O., S. 3

${ }^{63}$ Vgl. Kohlmeier, L. (1994), a.a.O., S. $264 \mathrm{ff}$.

${ }^{64}$ Da neben anderen Ermittlungsproblemen v.a. die Bekundung der (fiktiven) Zahlungsbereitschaft keine tatsächlichen Ausgaben nach sich zieht, ist anzunehmen, dass ggf. höhere oder unreali- 
Durchführbarkeit und Sinnhaftigkeit zu hinterfragen. Zur Berechnung der indirekten Kosten einer bestimmten Erkrankung für eine gesamte Volkswirtschaft wird in der Literatur zumeist auf den Humankapitalansatz verwiesen. ${ }^{65}$ In einer Bruttoeinkommensrechnung unselbständiger Erwerbstätiger werden demgemäß nach der Humankapital-Methode und auf Grundlage verlorener Erwerbstätigkeitsjahre die Gesamtverluste von Lebenseinkommen betroffener Patientengruppen - und damit die erzeugten indirekten Kostenanteile - auf Gegenwartswerte berechnet. Diesem Ansatz wird auch bei den hier durchgeführten Berechnungen gefolgt. Das genaue schrittweise und teils komplexere Vorgehen zur Ermittlung der indirekten Kosten für Arbeitsunfähigkeit, Invalidität und Mortalität wird im Ergebnisteil an den jeweiligen Stellen erläutert und mit Tabellen (teils im Anhang) unterstützt.

\subsubsection{Intangible Kosten}

Seitens der WHO gibt es seit den 90er Jahren des vergangenen Jahrhunderts im Rahmen des sog. „Burden of Disease“- bzw. „Environmental Burden of Disease“Ansatzes verstärkte Bemühungen, Maßzahlen zu etablieren, welche, wie z.B. die Maßzahl DALY (Disability Adjusted Life Years), einen Verlust von Lebensqualität quantifizieren. ${ }^{66}$ Auch Kosten, die bei Angehörigen von Erkrankten entstehen (Pflege, Leid usw.), stehen dabei im Blickpunkt und werden im gesundheitsökonomischen Sprachgebrauch zusammengefasst als „Intangible Kosten“ beschrieben. Mit ihrer Berücksichtigung verbände sich im Zusammenhang mit der Anforderung einer Gesamtkostenanalyse die Problematik, originär nicht monetär vorliegende Größen in weiteren Schritten schließlich doch monetär bewerten zu müssen ohne jedoch durchgängig bzw. ausschließlich - wie bei der Berechnung der indirekten Kosten - auf eine, wie die Bruttoeinkommensrechnung, einheitliche Bemessungsgrundlage zurückgreifen zu können. Hier kommt man daher schnell

stische Zahlungsbereitschaften von Patienten angegeben werden, als im Eintrittfalle tatsächlich geleistet würden oder aus finanziellen Gründen geleistet werden könnten.

${ }^{65} \mathrm{Vgl}$. Kohlmeier, L. et. al (1994), a.a.O. S. $251 \mathrm{ff}$. u. $291 \mathrm{f}$ z.B. Welte, R. et.al. (2004), a.a.O., S. 3 , http://www.helmholtz-muenchen.de/neu/Wir ueber uns/jahresbericht/2004/031 036 igm akt.pdf, wie auch Hauner, H. et al (2007), a.a.O., S. 50f.

${ }^{66}$ Die WHO fasst die von ihr entwickelten Maßzahlen dieser Art unter dem Begriff SMPH (Summary Measures of Population Health) zusammen. Sie werden als dynamische Größen 
in die ethische oder philosophische Diskussion um Werte, zumindest bei der Bewertung eines adäquaten Gegenwerts von Lebensqualität. Aufgrund der hohen Komplexität zur Ermittlung von intangiblen Kosten und der gegebenen Anfälligkeit für Subjektivität, finden sie in den heutigen Kostenberechnungen kaum konkrete Anwendung und werden, wie auch in diesem Fall, zumeist als integrierter Anteil der wesentlich bedeutsameren indirekten Kosten verstanden und in integrierter Weise als solche behandelt.

\subsubsection{Weitere zurechenbare Kosten}

Die Möglichkeit, weitere Kosten mit einzubeziehen, die über das bisher beschriebene Maß hinausgehen, ist durchaus gegeben. Dies hängt zum einen mit dem nicht einheitlich definierten Umfang der mit Adipositas assoziierten Erkrankungen zusammen. Alle dahingehenden Berücksichtigungen sind grundsätzlich daran zu messen, inwieweit sie mit vertretbarem Aufwand zu ermitteln sind, bzw. einen Mehrwert für die Zielsetzung und das Ergebnis der Analyse beitragen können. Es ist aufgrund unterschiedlicher Quellen/ Studien und medizinischer Beweislage durchaus möglich, beizumessende Anteile mit unterschiedlichen Werten anzugeben oder manche Erkrankungen entweder einzubeziehen oder aus der Betrachtung auszuschließen. Zum anderen ist zu bedenken, dass die Krankheitskausalitäten auf der Grundlage von Adipositas nicht mit jenen identifizierten Krankheiten ausreichend begründet sein müssen, die als erste, unmittelbare Folge der Fettleibigkeit entstehen. Manche Studien berücksichtigen darüber hinaus zweite oder dritte Stufen von Folgekosten durch Adipositas. ${ }^{67}$ Ein Beispiel wäre die Berücksichtigung des Ereignisses eines Schlaganfalls (2. Stufe), das nicht direkt durch Adipositas, sondern ggf. erst auf Grundlage einer Arteriellen Hypertonie (1. Stufe) entstanden sein kann, was die Komplexität der Zusammenhänge weiter erhöht, hier jedoch nicht umgesetzt wird. Die vorgestellten, im weiteren Verlauf angewendeten Methoden und Vorgehensweisen bedingen, dass in den zu treffenden Aussagen bzw. Ergebnissen notwendigerweise Unsicherheiten enthalten sind. Diese Unsicherheiten können dadurch relativiert werden, dass die verwen-

aufgefasst und gemäß neuen Anforderungen kontinuierlich weiterentwickelt. Vgl. Malsch, A. K. et. al. (2006), In: Materialien „Umwelt und Gesundheit“ Nr. 65.

${ }^{67}$ Z.B. Schneider, H./ Schmid, A. (2004), a.a.O., S. $43 f f$. 
deten Methoden bereits in ähnlichen, vergleichbaren Zusammenhängen oder in früheren veröffentlichten Analysen verwendet wurden und innen Akzeptanz und Aussagekraft auf nationaler und internationaler Forschungsebene zuzusprechen ist. 


\section{Ergebnisse}

\subsection{Kostenanalyse}

Die fehlende statistische Verfügbarkeit von Gesamtkosten der Adipositas, einerseits im Sinne von direkten und indirekten Kosten, andererseits von notwendigerweise zurechenbaren Kosten assoziierter Erkrankungen, erfordert eine schrittweise Ermittlung dieser einzelnen Kostenbestandteile. Zunächst werden dazu die direkten Kosten der Adipositas ermittelt bzw. auf Grundlage von recherchierten Basisdaten abgeschätzt und hochgerechnet. Im Anschluss daran werden ergänzend die direkten und indirekten Kosten von Folgeerkrankungen dargelegt. Zum Vergleich der Ergebnisse werden die existierenden Studien herangezogen.

\subsubsection{Direkt zurechenbare Kosten}

Es existieren unterschiedliche Studien, von denen für die hier gestellten Anforderungen einer Gesamtkostenanalyse keine einen überzeugenden Beitrag leisten kann. Direkte Krankheitskosten der Adipositas wurden z.B. im Rahmen der KORA-Studie als aufwendige Bottom-up-Untersuchungen durchgeführt. Dabei wurden aus repräsentativen Stichproben Pro-Kopf-Berechnungen angestellt und Durchschnittkosten ermittelt. ${ }^{68}$ Ein zentraler Aspekt bei der Interpretation der Ergebnisse für die hier zu klärende Fragestellung ist, dass das geforderte Ziel der Studie nicht darin bestand, direkte Kosten für Begleit- und Folgeerkrankungen der Adipositas herauszurechnen. Auch bestand keine Anforderung, eine Selektion und Priorisierung dieser Erkrankungen vorzunehmen, so dass die Hochrechnungen der "direkten medizinischen Kosten“ weitgehend unselektiert auf Basis aller Patientenangaben und somit nahezu aller Erkrankungen, wegen derer sie im Zusammenhang mit Adipositas einen Arzt (z.B. auch Zahnarzt) aufsuchten, durchgeführt werden konnten. Insofern kann die gliederungsbedingte Trennung zwischen direkten Kosten der Adipositas und direkten Kosten assoziierter Erkrankungen nicht eingehalten werden. Darüber hinaus werden aufgrund bestehender Beschränkungen die Ergebnisse der Studie als nicht geeignet dafür angesehen, Hochrechnungen bezüglich der Gesamtbevölkerung der Bundesrepublik Deutsch-

\footnotetext{
${ }^{68}$ Vgl. von Lengerke, T et al. (2006).
} 
land vorzunehmen. ${ }^{69}$ Des Weiteren kann auf die Gesundheitskostenrechnung des Bundes zurückgegriffen werden, die im Sinn einer Sekundärstatistik die aggregierten Krankheitskosten für die Bundesrepublik Deutschland zugeordnet zu den ICD 10-Ziffern vorhält. Jedoch verbinden sich damit wesentliche Defizite, zum einen dadurch, dass den Ergebnissen lediglich ein distributiver Top-down-Ansatz zugrunde liegt, zum anderen aufgrund der systematischen Nichtberücksichtigung zurechenbarer direkter Kosten für assoziierte Erkrankungen, um nur zwei der vordringlichsten zu nennen.

Insofern stellt sich die Notwendigkeit, eine aktuelle Ermittlung/ Schätzung der direkten Kosten hinsichtlich folgender Einzelbestandteile vorzunehmen:

1. Kostenbewertung der Adipositas-Medikamente, von denen gemäß Adipositas-Leitlinie bislang drei zugelassen waren: ${ }^{70}$

- Sibutramin (Reductil囚): ein selektiver Serotonin- und NoradrenalinWiederaufnahmehemmer, welcher v.a bei Personen mit Typ 2 Diabetes Gewichtssenkungen in Aussicht stellt.

- Orlistat (Xenica|®): ein im Gastrointestinaltrakt wirkender Lipaseinhibitor mit einem Gewichtssenkungspotential von im Mittel $3 \mathrm{~kg}$.

- Rimonabant (Acomplia ${ }^{\circledR}$ ): ein in das körpereigene (Endo-)Cannabinoid-System eingreifendes neuartiges Medikament, welches zur Gewichtsreduktion und zur Raucherentwöhnung eingesetzt wird. Nach Herstellerangaben kann bei einer über 2 Jahre angewendeten Dosis von $20 \mathrm{mg}$ pro Tag im Durchschnitt eine Gewichtsreduktion von fünf Prozent des Körpergewichts erzielt werden. ${ }^{71}$

2. Stationäre Behandlung von Adipositas; inklusive Reha-Maßnahmen, in erster Linie aufgrund der angewendeten Therapieformen der Wahl:

- Magenband-Operationen

- Magenbypass-Operationen

\footnotetext{
${ }^{69}$ Vgl. von Lengerke, T. et al. (2006), S. 114.

${ }^{70}$ Hauner, H. et al. (2007), S. $11 f$.

${ }^{71}$ Aufgrund von unkalkulierbaren Nebenwirkungen wurde das Produkt im Oktober 2008 bis auf Weiteres vom Markt genommen.
} 
3. Kosten der ambulanten fachärztlichen Behandlung von Adipositas, die sich auf Beratungsleistungen beschränkt (Therapie in erster Linie Folgeerkrankungen), mit den Aspekten:

○ Ernährungsberatung/ Diätplanung

- Beratung für Bewegungstherapie/ Sport, Psychologie

\section{Medikamentenkosten}

Die Kostenbewertung der (bislang) zugelassenen Adipositas-Medikamente stützt sich v.a. auf gängige Apothekenpreise (Marktpreise), wobei in der Pharmaindustrie, ausgehend von den Herstellungskosten der Medikamente, grundsätzlich von einer Marge von etwa 20-40\% auszugehen ist. Die Kosten einer Behandlung mit Orlistat in der Standard-Dosierung werden in einer Spanne von $45 €$ bis $85 €$ Marktpreis pro Monat angegeben. ${ }^{72}$ Sibutramin (Reductilß) ist als Kapseln zu 10 und zu $15 \mathrm{mg}$ erhältlich. Bei Einnahme der üblichen Tagesdosis belaufen sich die monatlich aufzubringenden Kosten auf durchschnittlich etwa $45 €$. bis $90 € .^{73}$ Rimonabant (Acompliaß) ist das jüngste der Adipositas-Medikament und mit Kosten von über $90 €$ auch das teuerste. Die Ermittlung der direkten Kosten kann bei Medikamenten, welche über die Krankenkassen erstattet werden, über die Krankenkassenroutinedaten abfragen. Dies ist im Falle der zugelassenen Adipositas-Medikamente nicht möglich, da diese nach § 34 SGB V von der Versorgung der gesetzlichen Krankenkassen ausgeschlossen sind. Der Versuch, genauere Informationen über die Umsatzanteile der Herstellerfirmen zu erhalten, war nur bedingt realisierbar, da die jeweiligen Pharmaunternehmen aus Wettbewerbsgründen wenig veröffentlichtes und verlässliches Datenmaterial bereitstellen. Erforderliche Hochrechnungen basieren daher auf einer äußerst restriktiven Datenbasis, was einer Bewertung und der Repräsentativität möglicher Ergebnisse klare Grenzen setzt. Grundsätzlich ist im Rahmen von Medikamentenverordnungen ein genereller Trend festzustellen, der zu steigenden Umsätzen bei rückläufigen Verordnungszahlen führt, was für eine Verteuerung der Medikamente

\footnotetext{
${ }^{72} \mathrm{Vgl}$. http://www.infomed.org/pharma-kritik sowie unterschiedlicher Online-Preisvergleichsportale, wie z.B. http://www.apomio.de.

${ }^{73}$ Vgl. ebenda.
} 
spricht. ${ }^{74}$ Laut einer Untersuchung der Deutschen Bank für die USA wurde eine Umsatzentwicklung von Adipositas-Medikamenten für das Jahr 2000 mit 0.7 Mrd. \$, für das Jahr 2005 mit 1.5 Mrd. \$ angegeben. Für das Jahr 2010 werden 2.2 Mrd. \$ vorausgesagt. ${ }^{75}$ ROCHE, der Hersteller von Xenical ${ }^{\circledR}$ hat für 2007 allerdings wieder rückläufige Umsätze angegeben. ${ }^{76}$ Die Informationslage zeigt, dass Uneinheitlichkeit und Unsicherheit in diesem Medikamentensegment herrscht, was die Kostenkalkulation wesentlich erschwert. Im Finanzbericht des Reductil@Herstellers BASF aus dem Jahr 2000 wurde letztmals ein Umsatz für Deutschland und die Schweiz zusammen für 1999 von 137 Mio. $€$ veröffentlicht. $^{77}$ Diese Umsatzgrößenordnung scheint in Anbetracht der gegenwärtigen Zahlen nicht mehr realisierbar zu sein. Aktuellere Umsatzzahlen werden - nach Rückfrage beim Hersteller - aus bereits erwähnten Wettbewerbsgründen nicht mehr publiziert. Im Falle von Xenical® stellt sich die Informationslage nicht besser dar, da ROCHE in seinem Geschäftsbericht von 2004 lediglich Zahlen für ganz Europa veröffentlicht und für 2004 einen gesamten EU-Umsatz von 593 Mio. SFr. angibt, was rund 395 Mio. $€$ entspricht. ${ }^{78}$ Auch die Firma Sanofi-Avensis, die das Produkt Acomplia ${ }^{\circledR}$ (Rimonabant) entwickelt hat, liefert keine veröffentlichten, zuverlässigen Umsatzdaten, die einen Rückschluss auf die Kosten im Markt zuließen. Im Zusammenhang mit diesem Medikament sind in jüngster Zeit zudem Probleme mit Nebenwirkungen aufgetreten, die zu einer (zumindest vorläufigen) Rücknahme des Produktes vom Markt geführt haben.

Einzig die Einrichtung IMS-Health verfügt über Statistiken über sog. „HumanArzneimittel-Einkäufe öffentlicher Apotheken“, über welche die Kosten der hier relevantern Medikamente nachvollzogen werden können. ${ }^{79}$ Danach lässt sich folgende Aufstellung vornehmen:

\footnotetext{
${ }^{74}$ Vgl. Paffrath / Schwabe (Hrsg.) (2007): AVR 2006.

${ }^{75}$ Deutsche Bank Research (2002) Nr. 244.

${ }^{76}$ ROCHE Wissenschaftlicher Pressedienst (2007).

${ }^{77}$ Vgl. BASF Finanzbericht 2000, S. $36 f$.

${ }^{78} \mathrm{Vgl}$. ROCHE Geschäftsbericht (2004), Abschnitt Pharma Ergebnisse, S. $20 \mathrm{ff}$.

${ }^{79}$ Vgl. IMS Health (2007): DPM - Der pharmazeutische Markt Deutschland. Statistik über HumanArzneimittel-Einkäufe öffentlicher Apotheken. Hierbei gilt der besondere Dank der Universität
} 
Tabelle 8: $\quad$ Einkaufskosten der Apotheken auf Basis der in Deutschland zugelassenen Adipositas-Medikamente Sibutramin, Orlistat und Rimonabant.

\begin{tabular}{|c|c|c|c|c|}
\hline Medikament & $\begin{array}{c}\text { Anzahl } \\
\text { Packungen }\end{array}$ & $\begin{array}{l}\text { Gesamtkosten } \\
\text { Einkauf (in } € \text { ) }\end{array}$ & $\begin{array}{c}\text { Marge } \\
\text { Apotheken } \\
\text { von } 40 \% \text { (in } € \text { ) }\end{array}$ & $\begin{array}{c}\text { Gesamtkoste } \\
\text { n im Markt (in } \\
€ \text { ) }\end{array}$ \\
\hline$\frac{\text { Sibutramin }}{\text { (Reductil®) }}$ & 308.200 & 15.730 .400 & 6.292 .160 & 22.022 .560 \\
\hline$\frac{\text { Orlistat }}{(\text { Xenical®) }}$ & 141.400 & 8.099 .200 & 3.239 .680 & 11.392 .880 \\
\hline$\frac{\text { Rimonabant* }}{\text { (Acomplia®) }}$ & 180.500 & 16.596 .000 & 6.638 .400 & 23.234 .400 \\
\hline Gesamt pro Jahr & 630.100 & 40.425 .600 & 16.224 .240 & 56.649 .840 \\
\hline
\end{tabular}

* Aufgrund von unkalkulierbaren Nebenwirkungen wurde das Produkt im Oktober 2008 bis auf Weiteres vom Markt genommen.

Quelle: $\quad$ IMS Health (2007): DPM - Der Pharmazeutische Markt Deutschland.

Geht man in der Folge von den o.g. Gewinnmargen der Apotheken aus und setzt diese mit 40\% an deren obere Grenze, dann ergeben sich insgesamt 56.649.840 $€$ an Kosten (Marktpreise) für die derzeit zugelassenen Adipositas-Medikamente in Deutschland. Es gilt dabei zu bedenken, dass neben diesen offiziell zugelassenen Medikamenten zahlreiche andere Produkte mit ähnlicher Zielsetzung im Markt vorhanden sind, die hier nicht berücksichtigt werden. Dies liegt zum einen an der fehlenden Verfügbarkeit diesbezüglicher Daten, zum anderen an dem oftmals fehlenden Nachweis einer therapeutischen Wirksamkeit. Hier ist der Übergang zu Diät- und Fitness-Produkten als fließend und kaum abgrenzbar anzusehen.

\section{Kosten für stationäre/ teilstationäre Behandlung}

Grundsätzlich unterliegt die Berechnung der stationären Behandlungskosten vergleichbaren Schwierigkeiten wie die Ermittlung der Medikamentenkosten im vorherigen Abschnitt. Auch hier finden sich wenige Datenquellen oder repräsen-

Bremen, Zentrum für Sozialpolitik Gesundheitsökonomie, Gesundheitspolitik und Versorgungsforschung und insbesondere Prof. Dr. Gläske und Mitarbeitern für die Erschließung dieser Quelle. 
tative Studien, so dass die Berechnungen nur auf einzelnen, selektierten Quellen beruhen, deren Verlässlichkeit durchaus kritisch zu sehen ist. Auch hier spielt es eine entscheiden Rolle, dass - mit wenigen Ausnahmen - der Patient die Kosten für einen operativ-therapeutischen Eingriff selbst zu tragen hat und dadurch die statistische Erfassung unzureichend ist. Trotz dieser Umstände wurde dennoch der Versuch einer (konservativen) Berechnung vorgenommen, um zumindest kalkulatorische Anhaltspunkte für dieses Kostensegment zu liefern. Es wird als dringend erforderlich angesehen, systematisch die Kostentransparenz in diesem Bereich zu erhöhen und tragfähige Daten zu generieren und diese zu publizieren.

Die Berechnung der stationären Behandlungskosten von Adipositas erfolgt anhand von Zahlenangaben auf der Grundlage des geltenden Krankenhausentgeltgesetzes. In Frage kommende Eingriffe, wie Magenbypassoperationen/ Magenverkleinerungstechniken (vertikale Gastroplastik und Silikonbandtechnik), werden als Mittel der Wahl der gegenwärtigen invasiven Therapiemaßnahmen angesehen. ${ }^{80}$ Diese Operationsformen werden i.d.R. für Patienten mit exzessiver Fettleibigkeit und einem BMI > 40 angewendet. Problematisch für eine einheitliche Berechnung ist, dass die Operationen teils ambulant/ teilstationär durchgeführt werden und nicht immer die reguläre stationäre Aufnahme der Betroffenen erfolgt. Auch werden in allen Fällen zwar postoperative Nachuntersuchungen durchgeführt, eine Rehabilitation ist eher seltener die Regel, jedoch nur bedingt nachvollziehbar. Insofern werden vereinfachend die Kosten für anfallende Nachuntersuchungen und ggf. vorgenommene Reha-Maßnahmen als eine zusammengefasste Kostengröße behandelt.

Zahlen über Operationen zum Zwecke der Behandlung von Adipositas sind kaum zu erschließen. Dennoch sei gemäß einer Einzelerhebung und aktuellen Chirurgie-Kongressergebnissen für das Jahr 2005 von rund 1200 operativen Eingriffen in Deutschland auszugehen, was eine jährliche Operationsfrequenz von weniger

\footnotetext{
${ }^{80}$ Hauner, H. et al. (2007), S. 45.und Schneider, H. et al. (2004), S. $16 \mathrm{ff}$.
} 
als $0,1 \%$ in Bezug auf die potenziell operablen Personenkreis bedeute. ${ }^{81}$ Dieser Wert kann wohl als eher restriktiv angenommen werden, da von einer gewissen Grauzone auszugehen ist, die aufgrund von i.d.R. nicht erstattungsfähigen Abrechnungsbedingungen existiert. Daraufhin liegt es nahe, dass sich bietende Möglichkeiten genutzt werden, derartige Operationen im Zuge anderer gleichzeitig erforderlicher Therapie von Begleit- oder Folgeerkrankungen und somit unter anderen Diagnosen/ Abrechnungsmöglichkeiten durchzuführen und zu erfassen. Um bei Zunahme der Adipositas-Prävalenz in den nächsten Jahren eine vergleichbare Operationsfrequenz wie in den USA zu erzielen (2006: 250.000), wird hochgerechnet, dass eine Steigerung der Operationszahlen in Deutschland um den Faktor 20-40 erforderlich sein dürfte. ${ }^{82}$ Auch in diesem Zusammenhang liegen kaum Informationen auf aggregiertem Niveau vor, welche die o.g. Schätzungen ggf. untermauern könnten.

Die direkten Kosten eines stationären/ teilstationären Aufenthalts setzen sich insgesamt zusammen aus den Bestandteilen:

- Kosten der Operation (teilstationär oder stationär)

- Fallpauschale (mit Bezug auf die mittlere Klinikverweildauer)

- Krankenhausindividuelle Zusatzentgelte (nach Anlage 3a KFPV)

- Nachbehandlung (grundsätzlich)/ Reha-Kosten (seltener)

Da nicht zu ermitteln ist, wie viele der Eingriffe von Belegärzten teilstationär oder in Kliniken stationär vorgenommen werden, wird vereinfachend davon ausgegangen, dass alle behandelten Patienten eine "mittlere Verweildauer“ gemäß § 1 Abs. 6 der Verordnung zum Fallpauschalensystem für Krankenhäuser (KFPV) von etwa 7 Tagen aufweisen. ${ }^{83}$ Die im Fallpauschalenkatalog unter der Diagnosis Related

\footnotetext{
${ }^{81}$ Vgl. Weiner, R. A. (2007). Zudem wurde auf dem 125. Kongress der Deutschen Gesellschaft für Chirurgie 2008 in Berlin analysiert, dass gerade einmal 64 Kliniken in Deutschland Adipositaschirurgie betreiben oder in der Vergangenheit betrieben haben. Nur 13 Kliniken hätten Erfahrungen auf diesem Fachgebiet von mehr als 7 Jahren und nur 3 Kliniken seit mehr als 15 Jahren, was den aktuell noch geringen Stellenwert untermauert.

${ }^{82}$ Weiner, R. A. (2007), in Verbindung mit Stroh, C. et al. (2007), Ergebnisse der Pilotphase 2005.

${ }^{83}$ Der Fallpauschalen-Katalog gibt für stationäre Adipositas-Patienten 7,8 Tage und für teilstationäre 6,3 Tage an. Der diesbezügliche Bundesdurchschnitt liegt bei 8,7 Tagen.
} 
Group (DRG) geführte Ziffer K07Z „Andere Eingriffe bei Adipositas“ repräsentiert dabei den Gegenstand einer stationären Versorgung durch Klinikhaupt- bzw. Belegabteilungen. Die zugrunde zu legenden Krankenhaus-Erlöse ergeben sich aus dem Produkt von Basisfallpreis und dem jeweiligen DRG-Relativgewicht. Da sich die Basisfallpreise von Klinik zu Klinik unterscheiden, werden hier die bundesdurchschnittlichen Kosten je Behandlungsfall herangezogen, die $3.218 €$ betragen. ${ }^{84}$ Des Weiteren sieht die Verordnung sog. „krankenhausindividuelle Entgelte" vor, die jede Klinik selbst festlegen kann. Auch hier wurde ein Durchschnittswert angenommen, der sich aus verschiedenen Stichproben bei einzelnen Kliniken ergab. Unter Berücksichtigung der genannten Zusammenhänge lässt sich folgende Berechnung vornehmen:

Tabelle 9: $\quad$ Kalkulation der stationären/ teilstationären Kosten im Falle der Adipositas-Eingriffe erster Wahl

\begin{tabular}{|c|c|c|c|}
\hline & DRG Relativgewicht & Basisfallpreis & Gesamtkosten \\
\hline & Verrechnungssatz & $\ln €$ & $\operatorname{In} €$ \\
\hline Klinikhauptabteilung & 1,383 & 3.218 & $4.450,5$ \\
\hline Klinikbelegabteilung & 1,080 & 3.218 & $3.475,4$ \\
\hline $\begin{array}{c}\text { Krankenhausindividuelle } \\
\text { Entgelte (Anlage 3a KFPV) }\end{array}$ & -- & ca. $300 €$ bis $3500 €$ & 1.900 \\
\hline
\end{tabular}

Quelle: $\quad$ Fallpauschalenkatalog G-DRG Version 2007.

Aus dieser Aufstellung ergeben sich gesamte kalkulierte Kosten einer stationären bzw. teilstationären Behandlung von $6.350 €$ bzw. $5.375 €$. Im Mittel ergeben sich demnach in etwa $5.863 €$ an Klinikkosten für die operative Adipositas-Therapie.

Zwar gelten Reha-Aufenthalte nach Adipositas-Operationen eher als selten, doch werden hier annahmegemäß unter den Reha-Kosten alle Kosten für Nachsorgeuntersuchungen (v.a. auch ambulante) subsumiert, da hierfür keine gesonderten Daten vorgefunden wurden. Unter den einzelnen Reha-Einrichtungen herrscht im

\footnotetext{
${ }^{84}$ Statistisches Bundesamt (2005) in Verbindung mit dem HKG-Report (2005) der Hessischen Krankenhausgesellschaft e.V.
} 
Bezug auf die Kosten eine hohe Variationsbreite. Seitens des deutschen Rentenversicherungsbundes wird von einer durchschnittlichen Fallpauschale von $3.100 €$ (2004) ausgegangen, die sich deutlich unter der eines kalkulierten (teil)-stationären Klinikaufenthalts $(5.863 €)$ bewegt. ${ }^{85}$ Insofern stellen sich die Kostenbestandteile der stationären Behandlung mit Bezug auf 1.200 Behandlungsfälle pro Jahr wie folgt dar:

Tabelle 10: $\quad$ Kalkulierte stationäre Kosten für die operative Behandlung der Adipositas (Operationen der Wahl)

\begin{tabular}{|c|c|c|}
\hline $\begin{array}{c}\text { Stationäre } \\
\text { Behandlungskosten }\end{array}$ & $\begin{array}{c}\text { Einzelkosten als } \\
\text { Durchschnittswert } \\
\text { (in } € \text { ) }\end{array}$ & $\begin{array}{c}\text { Bei } 1.200 \\
\text { Behandlungsfällen } \\
\text { (in } € \text { ) }\end{array}$ \\
\hline Stationärer Klinikaufenthalt & 5.863 & 7.035 .600 \\
\hline Nachbehandlung/ Reha & 3.100 & 3.720 .000 \\
\hline Summe & $\mathbf{8 . 9 6 3}$ & $\mathbf{1 0 . 7 5 5 . 6 0 0}$ \\
\hline
\end{tabular}

Quelle: $\quad$ Eigene Berechnungen auf Grundlage der angegebenen Quellen.

Die als hoch einzuschätzenden Unsicherheiten bei diesen Berechnungen sind weniger in den Preisen der Gesundheitsleistungen zu sehen als vielmehr in den Mengenangaben. Mit 1.200 Operationen pro Jahr und einer Kostengröße von rd. 10,76 Mio. € wird annahmegemäß eine konservative Zahl vorgestellt. Unterstellte man zusätzlich - was über vorhandene Informationen allerdings nicht belegbar ist - dass weitere Operationen und ambulante Beratungen zwar durchgeführt, jedoch z.B. aus Abrechnungsgründen unter anderen ICD 10-Ziffern dokumentiert würden, käme der bereits erwähnte Kostengraubereich zum Tragen, der ggf. im Rahmen der Behandlung anderer Begleiterkrankungen zu suchen wäre und unter anderen Diagnoseziffern zu Buche schlagen würde. Derartige Aspekte sind zwar als wahrscheinlich anzunehmen, können in diesem Untersuchungsrahmen gleichzeitig jedoch nicht konkret belegt oder gar beziffert werden.

\footnotetext{
${ }^{85}$ Reimann, A. et al. (2007) Presse-Vortrag.
} 


\section{Kosten für ambulante Behandlung (ohne Operationen)}

Schließlich sind die Kosten der ambulanten, fachärztlichen Behandlung/ Beratung von Adipositas zu betrachten, die v.a. die Ernährungsberatung, Diätplanung, Bewegungstherapieberatung betreffen. Die relevanten ambulanten Adipositasprogramme werden dabei an umfassenden Qualitätsanforderungen gemessen. ${ }^{86}$ Häufig werden diese Beratungen in Kooperation mit Ernährungsfachkräften, Psychologen sowie im Rahmen der Bewegungstherapie von Physiotherapeuten durchgeführt, so dass diese hinzuzuzählen sind. Die Verrechnungssätze für Beratungen können nach Erst- und Folgeberatung aufgeteilt werden und belaufen sich auf aktuell $61,20 €$ (Erstberatung) bzw. 30,59€ (Folgeberatung) ${ }^{87}$

Konkrete Zahlen und Auswertungen über tatsächlich durchgeführte Ernährungsberatungen sind gegenwärtig nicht zu ermitteln, so dass die Kosten nur vage abgeschätzt werden können. Ein wesentlicher Teil der Beratungskosten ist im Zusammenhang mit den Kosten der Folgekrankheiten (v.a. Diabetes mellitus) zu sehen, da Arztbesuche v.a. aufgrund von damit zusammenhängenden Beschwerden erfolgen. Lediglich in einer veröffentlichten Untersuchung wurden ausgehend von einer (auf Ernährungsberatung spezialisierten) Arztpraxis (inkl. Kooperationen) in einem Jahr 112 Erst- und 76 Folgeberatungen durchgeführt, wodurch sich gemäß o.g. Verrechnungssätze ein Betrag von rund $9.180 €$ pro Jahr ermitteln lässt. ${ }^{88}$ Extrapoliert man diesen Wert auf ca. 2000 ambulante ärztliche Stellen mit Ernährungsberatungskompetenz in Deutschland, ${ }^{89}$ dann ergeben sich derzeit Ernährungsberatungskosten in Höhe von etwa 18,36 Mio. €. pro Betrachtungsjahr. Auch diese Ergebnisse sind - wie jene der vorangegangen Abschnitte - mit großer Unsicherheit verbunden, deren Repräsentativität kritisch ist. Die Defizite der Datenverfügbarkeit schränken eine fundierte Erhebung der direkten Kosten für Adipositas daher wesentlich ein, was es bei der Interpretation der Ergebnisse zu bedenken gilt.

\footnotetext{
${ }^{86}$ Hauner et al. (2000), S. 5-8.

${ }^{87}$ Winckler, K. (2005), S. 30-42.

${ }^{88} \mathrm{Vgl}$. ebenda.

${ }^{89}$ Vgl. Angaben der Ärztekammern (2007) bzw. der Bundesärztekammer.
} 
Die insgesamt ermittelten direkten Kosten können durch Addition der einzeln ermittelten Kostenbestandteile Medikamente, stationäre Kosten und ambulante Kosten nun beziffert werden und belaufen sich zusammen auf geschätzte $\underline{85,71}$ Mio. $€$. In der Übersicht stellen sie sich wie folgt dar:

Abbildung 5: $\quad$ Direkte Kosten der Adipositas in der Bundesrepublik Deutschland (virtuelles Bezugsjahr)
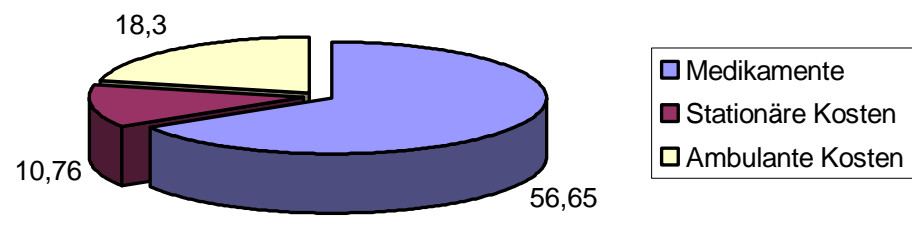

$\square$ Ambulante Kosten

Quelle: $\quad$ Eigene Darstellung auf Basis der durchgeführten Einzelberechnungen.

Es zeigt sich ein klares Übergewicht zugunsten der Medikamentenkosten, die mit einem Anteil von 66,10\% anfallen. Mit 21,35\% folgen die Kosten für die ambulante Behandlung der Adipositas, während die stationären Kosten mit 12,55\% den geringsten Anteil repräsentieren. ${ }^{90}$

In der Gesamtdarstellung aller zum Zwecke der Ermittlung direkter Krankheitskosten von Adipositas herangezogener Studien und Berechnungen, ergibt sich ein vermutetes heterogenes Bild bei Uneinheitlichkeit der Ergebnisse, die zudem aufgrund von Datenverfügbarkeiten, methodischer und erhebungsspezifischer Unterschiede als nur bedingt vergleichbar anzusehen sind.

\footnotetext{
${ }^{90}$ Im Zuge der BMG-Erhebungen von Kohlmeier , L. (1993) wurden zu diesem Zeitpunkt gerade einmal 1 Mio. DM an Medikamentenkosten identifiziert, dagegen nicht nachvollziehbare ambulante und stationäre Kostenanteile in Höhe von 200 Mio. DM bzw. 131 Mio. DM.
} 
Tabelle 11: Direkte Krankheitskosten auf Basis unterschiedlicher Erhebungen in der Übersicht

\begin{tabular}{|c|c|c|}
\hline Datenquelle/ Studie & Bezug der Ergebnisse & $\begin{array}{l}\text { Direkte Kosten der Adipositas } \\
\text { per se } \\
\left(\mathrm{BMI}>30\left[\mathrm{~kg} / \mathrm{m}^{2}\right]\right)\end{array}$ \\
\hline Unterschiedliche Jahre & & Angaben in $€$ \\
\hline $\begin{array}{l}\text { Eigene Berechnungen } \\
\text { („virtuelles“ Jahr) }\end{array}$ & $\begin{array}{l}\text { Siehe Quellen und } \\
\text { Vorgehensweise }\end{array}$ & 85,71 Mio. $€$ \\
\hline Kohlmeier, L. (1993) & $\begin{array}{c}\text { Kosten ernährungsbedingter } \\
\text { Erkrankungen } \\
\text { (BMG-Kalkulation) }\end{array}$ & $\begin{array}{l}\text { 169,75 Mio. } € \\
\text { (332 Mio. DM) }\end{array}$ \\
\hline $\begin{array}{l}\text { KORA Survey 1999/ } 2000, \\
\text { von Lengerke et al. }\end{array}$ & $\begin{array}{l}\text { Durchschnittskosten/ Kopf } \\
\text { (bottom-up) }\end{array}$ & $\begin{array}{l}\text { Zwischen } 1.080 \text { bis } 2.572 € \\
\text { (BMI-anbhängig) pro Kopf und } \\
\text { Jahr (keine Gesamtkosten) }\end{array}$ \\
\hline $\begin{array}{l}\text { Kurscheid/ Lauterbach } \\
1998\end{array}$ & $\begin{array}{l}\text { aggregierte Kosten } \\
\text { (top down) }\end{array}$ & $\begin{array}{l}850 \text { Millionen } € \\
\text { pro Jahr }\end{array}$ \\
\hline Sander/ Bergemann 2003 & $\begin{array}{l}\text { aggregierte Kosten } \\
\text { (top down) }\end{array}$ & $\begin{array}{l}216 \text { Millionen } € \\
\text { pro Jahr }\end{array}$ \\
\hline $\begin{array}{l}\text { Gesundheits- } \\
\text { kostenrechnung d. Bundes } \\
2004\end{array}$ & $\begin{array}{c}\text { Aggregiert mit Bezug auf ICD } 10 \\
\text { Ziffern }\end{array}$ & $\begin{array}{l}717 \text { Millionen } € \\
\text { Gesamt pro Jahr }\end{array}$ \\
\hline
\end{tabular}

Quelle:

Eigene Zusammenstellung in Anlehnung an Aidelsburger, P. (2006): Die ökonomische Bedeutung der Adipositas für das deutsche Gesundheitssystem.

Die aggregierten direkten Kosten aus der Studie von Kurscheid/ Lauterbach weisen Kosten mit 850 Millionen $€$ pro Jahr auf ähnlich hohem Niveau aus, wie die 717 Millionen $€$, welche im Rahmen der Gesundheitskostenrechnung des Bundes ermittelt wurden. Sander/ Bergmann mit 216 Millionen $€$ und Kohlmeier mit (umgerechnet) 170 Mio. $€$ bleiben um rund das 3,5 bis 5 -fache unter diesen Werten. Die durchgeführten eigenen Berechnungen bewegen sich mit 85,71 Mio. $€$ deutlich unter allen vorliegenden Studienergebnissen, wenngleich die Vergleichbarkeit am ehesten mit der Studie von Kohlmeier (1993) gegeben ist, da von zumindest ähnlicher Vorgehensweise auszugehen ist. ${ }^{91}$

\footnotetext{
${ }^{91}$ Diesbezüglich weiterführende Aspekte werden im Diskussionsteil der Arbeit angeführt.
} 
Auffällig ist eine teils beträchtliche Abweichung der Kostenaufteilung zwischen Medikamenten-, ambulanten und stationären Kosten. V.a. das Zustandekommen der wesentlich höheren Kosten für ambulante Versorgung und stationäre Aufenthalte in den Vergleichsstudien ist mit Blick auf die Datenverfügbarkeit und herrschende Kostenstruktur kaum nachvollziehbar. Möglicherweise konnte in den Studien eine klare Trennung zwischen Adipositas und assoziierten Erkrankungen nicht eingehalten werden oder es wurden ggf. Kalkulationen mit deutlich höheren Ausgangswerten vorgenommen. Aus veröffentlichten Informationen konnte jedenfalls keine höhere Datendichte erschlossen werden. Die genauen und detaillierten Hintergründe für die teilweise erheblichen Unterschiede sind hier leider nicht abschließend zu klären.

Tendenziell kann für die eigenen Berechnungen aber die Schlussfolgerung gezogen werden, dass aufgrund der äußerst begrenzten Verfügbarkeit von Basisdaten, eine weitestgehend vorsichtige Schätzung vorgenommen werden musste und dass bestimmte, umfassendere Kostenbestandteile, wie z.B. Verwaltungsund Personalkosten beispielsweise im Vergleich zu den Berechnungen des Bundes nicht eingeflossen sind. Zudem fallen bei den durchgeführten Berechnungen Kosten jener Arztbesuche heraus, die unabhängig von Operationen oder Ernährungsberatungen, vorgenommen wurden, wie z.B. Sprechstundebesuche, die im Zuge der mit Adipositas assoziierten Erkrankungen (und somit unter anderen Diagnoseziffern als Adipositas) erfolgten. Es wurde eine bewusst strenge Abgrenzung zu den assoziierten Erkrankungen vorgenommen und nur tatsächlich nachvollziehbare Kosten der Diagnose Adipositas einberechnet. Inwieweit diese Abgrenzung in den übrigen Studien eingehalten wurde bzw. aufgrund des Studiendesigns eingehalten werden konnte bleibt offen. Ebenso finden Kosten für Gruppentherapien, Selbsthilfeprogramme, Hilfsmittel und Kosten für Medikamente/ Ernährungszusatzprodukte über das zugelassenen Maß hinaus, in den eigenen Berechnungen keine Berücksichtigung. Dadurch werden die Schwierigkeiten der Kostenzuteilung und gleichzeitig der als problematisch zu bewertende Spielraum der Analysemöglichkeiten offensichtlich und drücken sich konkret in Zahlen und variablen Ergebnissen aus. Insgesamt ist das Resultat dieses Berechungsabschnittes somit wenig zufriedenstellend. Der Umstand jedoch, dass 
(wie noch berechnet wird) der relative Anteil dieses Kostensegments nur in marginalem Maße ins Gewicht fällt (0,67\% der ermittelten Gesamtkosten), wirkt sich mit Blick auf die Repräsentativität der Gesamtkosten positiv aus. Bei höherem Anteil wäre diese Teilberechnung sicherlich nicht tragfähig genug.

Sowohl auf die Berechnung der direkten und hochsignifikanten Kosten der Begleit- und Folgeerkrankungen von Adipositas als auch auf die Ermittlung und Analyse der indirekten Kosten wird in den folgenden Abschnitten konkret eingegangen.

\subsubsection{Direkte Kosten assoziierter Erkrankungen}

Der Schwerpunkt der hier durchgeführten Berechnungen wird auf die bestehenden, möglichst aktuellen Einzelstudien der jeweiligen als assoziiert identifizierten Erkrankung gelegt. Die darin ermittelten direkten Kosten werden dann ebenfalls mit den als geeignet angesehenen beizumessenden Anteilen bewertet und können daraufhin beziffert werden. In den betrachteten Vergleichsstudien wurden - mit Ausnahme der KORA-Studie - ebenfalls Kosten aufgrund von Folge- und Begleiterkrankungen einbezogen. ${ }^{92}$ Auch wenn die offizielle Statistik des Bundes diesen Berechnungsschritt nicht in Betracht zieht, lässt er sich mit Hilfe beizumessender Anteile dennoch nachträglich durchführen, wodurch eine zusätzliche und wertvolle Vergleichsgröße der direkten Kosten von assoziierten Erkrankungen entsteht. Ein weiterer Grund, der für diesen Vergleich spricht, liegt darin, dass in jenen Fällen von assoziierten Erkrankungen, in denen keine Kostenstudien ermittelt werden konnten, ersatzweise die offiziellen Zahlen der Bundesstatistik herangezogen werden. Aufgrund der begrenzten Datenlage ist die eigene Berechung somit notwendigerweise auf diese offiziellen Angaben angewiesen.

Der zentrale Aspekt der assoziierten Erkrankungen ist in dem klinischen Vollbild des Metabolischen Syndroms zu sehen, womit eine Reihe gemeinsam auftretender Gefäßrisikofaktoren in Verbindung gebracht werden. Alle diesbezüglichen Aspekte stehen demnach in enger Beziehung zur Gruppe der arteriosklerotischen 
Gefäßerkrankungen. ${ }^{93}$ Zunächst erfolgt die konkrete Einschätzung der Bedeutung und des Risikos der im Rahmen von Adipositas identifizierten assoziierten Erkrankungen (vgl. Tabelle 4) anhand von ICD 10-Ziffern. In einem weiteren Schritt werden die direkten Kosten ermittelt, die aufgrund dieser Erkrankungen für das Gesundheitswesen entstehen. Daran anschließend wird der Blick auf die Identifikation der beizumessenden Anteile gerichtet, die dann eine gewichtete Kostenzuordnung der Einzelerkrankungen auf die Adipositas ermöglichen.

\section{Hypertonie (I $10-\mid$ 15)}

Der Bluthochdruck gilt als eine der häufigsten assoziierten Erkrankungen von Adipositas. Die Inzidenz für diese Folge- und Begleiterkrankung beträgt rund das Dreifache derer von Personen mit Normalgewicht. Bei Gewichtszunahmen von 20\% steigt Studien zufolge das Risiko für die Entstehung des Bluthochdrucks adipöser Personen sogar um das Achtfache. ${ }^{94}$ Die genauen Zusammenhänge gelten noch nicht als eindeutig geklärt. Es werden sowohl metabolische (Veränderungen der Insulin- und Zytokinproduktion) als auch hämodynamische (z.B. durch Gefäßveränderungen gestörte Fließeigenschaften des Blutes) Ursachenaspekte angeführt. In der sog. DOMS-Studie wurde zudem der Zusammenhang zwischen BMI und Mortalitätsrisiko untersucht und auch diesbezüglich wurden positive Korrelationen festgestellt. ${ }^{95}$

\section{Diabetes Mellitus (E $11-$ E 14)}

Die engste Assoziation zu Adipositas weist der Diabetes mellitus auf. Ausgehend von rund 5,7 Mio. Diabetikern seit 2001 wird von einer jährlichen Zunahme um ca. $5 \%$ ausgegangen. Der Anteil an Typ 2 Diabetikern beträgt dabei etwa 90\%. Allerdings lässt die Datenbasis keine exakte Differenzierung zwischen dem Typ 1 und Typ 2 zu. $^{96}$ Die gleiche Schwierigkeit besteht bezüglich der durch diese Erkrankung entstehenden Ausfallzeiten, die für eine Ermittlung indirekter Kosten

\footnotetext{
${ }^{92}$ Hinsichtlich der methodischen Detaillierung von Art und Umfang dieser Berechnungen wird auf die jeweilige Studie verwiesen.

${ }^{93}$ Hauner, H. et al. (2007), S. $25 \mathrm{ff}$.

${ }^{94} \mathrm{Vgl}$. Hubert et al. (1983): Frahamingham Heart Study \& Offspring Study.

${ }^{95}$ Bender et al. (2002), S. 239-245.

${ }^{96}$ Köster, I./ Hauner, H. (2006): CoDiM Study, S. 1499.
} 
relevant sein werden, so dass beide Diabetesformen in die Erhebung mit einflieBen. Aufgrund von hohen Zuwachsraten an adipösen Personen ist darüber hinaus mit weiter steigenden Zahlen v.a des Typs 2 zu rechnen. ${ }^{97}$ Laut der Nurses Health Study ist das Risiko an Diabetes zu erkranken bei adipösen je nach Ausprägung zwischen 20 und 60 Mal höher als bei Personen mit Normalgewicht. Der medizinische Hauptaspekt des Zusammenhangs wird in einer Hyperinsulinämie gesehen, die durch eine Erhöhung von freien Fettsäuren im Blut hervorgerufen wird, u.a. den Glukoseverbrauch des Muskels stört und eine vermehrte Insulinausschüttung - bis zur Insulinresistenz - hervorruft. ${ }^{98}$

\section{Kardiovaskuläre Erkrankungen (Ischämische Herzkrankheiten/ Herzinsuffizienz/}

Schlaganfall) (I 20 - I 25 und I 50)

Kardiovaskuläre Erkrankungen, wie Herzinfarkte oder Schlaganfälle, werden im Zusammenhang mit Adipositas nach neueren Erkenntnissen nicht mehr wie bislang nur in direkter Abhängigkeit von Diabetes, Hypertonie und erhöhten Blutfettwerten gesehen, sondern treten auch unabhängig davon auf. ${ }^{99}$ Die Adipositas nimmt z.B. negativen Einfluss auf das Entstehen und den Verlauf eines Herzinfarktes, so dass die Akutsterblichkeit von adipösen Patienten bei Herzinfarkt gesteigert ist und im Falle des Überlebens längere Klinikaufenthalte sowie in der Folgezeit häufigere Klinikeinweisungen erforderlich werden. ${ }^{100}$ Grundsätzlich wird mit dem Vorliegen von Adipositas die Tendenz eines Anstiegs des Gesamtcholesterins und der Triglyzeride in Verbindung gebracht sowie eine Reduktion des High-density Cholesterins HDL. Inwieweit das Überangebot an Nahrungsfetten oder tatsächlich manifestierte Fettstoffwechselstörungen eine Rolle spielen, ist nicht klar abzugrenzen. Als sicher wird angesehen, dass bei Personen mit einem $\mathrm{BMI}>30$ das Risiko für Blutfettwerte außerhalb der Normwerte um das Doppelte bis Dreifache steigt. Dadurch wiederum steigt die Gefahr an Atherosklerose zu erkranken und weitere, teils tödlich verlaufende Komplikationen des Gefäß-

\footnotetext{
${ }^{97}$ Ebenda, S. 1503.

98 Vgl. Colditz et al. (1990), Nurses Health Study, in Verbindung mit den umfassenden Ausführungen von Wirth, A. (o. J.) unter http://www.ifap.de/bda-manuale/adipositas/risiken.

${ }^{99}$ Vgl. Wirth, A. (o. J.) a.a.O. im Zusammenhang mit Schneider H. et al. (2004), a.a.O., S. 12.

${ }^{100}$ Wirth, A. (o. J.), im Zusammenhang mit der dort zitierten Frahamingham-Studie, a.a.O.
} 
systems zu fördern. ${ }^{101}$ Insofern werden die diesbezüglichen Risiken im Rahmen der kardiovaskulären Erkrankungen abgehandelt, auch weil die Datenlage zu diesem Thema sehr spärlich ist.

Neubildungen/bösartige Neubildungen (C 00 - C 97 selektiv)

Im Bezug auf Neubildungen/ bösartige Neubildungen besteht in der Literatur Uneinheitlichkeit darüber, welche spezifischen, insbesondere tödlich verlaufenden, Krebserkrankungen definitiv auf das Bestehen von Adipositas zurückgeführt werden können. Grundsätzlich werden deutlich erhöhte Risiken für die Entstehung von Kolon- und Prostatakarzinom, Brustkrebs, Nieren- und Gallenblasenkarzinom als durchaus wahrscheinlich angenommen. ${ }^{102}$ Neuere Studien aus den USA haben v.a. im Rahmen des Zusammenhangs von morbider Adipositas (BMI > 40) und tödlich verlaufenden Krebserkrankungen festgestellt, dass bei Männern über $50 \%$ und bei Frauen über $60 \%$ höhere Risiken im Vergleich zu Normalgewichtigen bestehen. Darüber hinaus wurde herausgefunden, dass bei jeder untersuchten Krebserkrankung mit steigendem BMI der Betroffenen ein tödlicher Verlauf der Erkrankung in ebenfalls steigendem Maße begünstigt wird. ${ }^{103}$ Für die hier durchgeführte Analyse werden die aus Kostensicht relevantesten Neubildungen herangezogen, wie das Kolonkarzinom (ICD $10 \mathrm{C18}$ ), die bösartigen Neubildungen der weiblichen Genitalorgane (C 51 - C 58) sowie bösartige Neubildungen der Prostata (C 61), was eher einer konservativeren Sichtweise auf Grundlage einer weitgehend gesicherten Studienlage gerecht wird. Trotz hoher Inzidenzen bösartiger Neubildungen, v.a. von Kolon- und Prostatakarzinomen, sind Kostenanalysen für Deutschland so gut wie nicht vorhanden.

Krankheiten der Gallenblase/ der Gallenwege und des Pankreas (K $80-\mathrm{K} 87$ ) Aus internistischer Sicht wird davon ausgegangen, dass mit übermäßiger Fettleibigkeit ein deutlich erhöhtes Risiko zur Bildung von Gallensteinen besteht, welches sich bei einem BMI von 30 bereits verdoppelt und bei stärker adipösen Personen bis zum Fünffachen ansteigt. ${ }^{104}$ Da wiederum rund $45 \%$ der diagnosti-

\footnotetext{
${ }^{101}$ Assmann/ Schulte (1993) im Rahmen der Münster Heart Study (PROCAM).

102 Schneider H. (2004), a.a.O., S. 13.

${ }^{103}$ Calle, E. et al. (2003).

${ }^{104}$ Wirth, A. (o. J.), a.a.O.
} 
zierten Pankreasnekrosen mit Gallensteinen vergesellschaftet sind, besteht bei bestehender Adipositas grundsätzlich ein erhöhtes Risiko, neben Gallen- und Gallenwegserkrankungen auch Erkrankungen des Pankreas zu begünstigen. Hierbei spielen neben mechanischen wiederum auch metabolische Störungen, wie z.B. eine Hyperlipidämie, eine maßgebliche Rolle. ${ }^{105}$

Arthrose (M $15-\mathrm{M} 17)$

Die Osteoarthrose als degenerative Gelenkerkrankung im Zusammenhang mit Adipositas imponiert in erster Linie über vermehrte Fälle von Hüft- und Kniegelenksarthrosen. ${ }^{106}$ Zusätzlich zu den durch die Wechselwirkung zwischen Übergewicht und Gelenkdestruktion entstehenden, mechanischen Überlastungen, werden systemische, metabolische Effekte auf die Entstehung der Osteoarthrose auch weiterer Gelenke als sehr wahrscheinlich angenommen. Insofern sollte es vertretbar zu sein, je nach Datenlage ggf. alle unter ICD 10 bezeichneten Arthrosen (M 15 - M 19) zur Kostenberechnung zu berücksichtigen.

\section{Psychosoziale Komplikationen (Depression) (F 32 - F 34)}

Im Falle der Kausalität von Adipositas und Depression besteht kein durchgängig einheitlicher Konsens darüber, ob Adipositas durch Depressionen oder umgekehrt Depressionen aufgrund von Adipositas hervorgerufen werden. Sicher scheint lediglich der Zusammenhang als solches zu sein, der in unterschiedlichen, v.a psychiatrischen, Studien untersucht wurde. ${ }^{107}$ Es zeigt sich im Speziellen, dass Depressionen vermehrt bei Personen mit v.a. höhergradiger Adipositas (class 2 und 3) auftreten, häufig gemeinsam mit einem allgemein reduzierten Gesundheitszustand. ${ }^{108}$ Dies lässt eine Berücksichtigung der Depression als kausaler Kostenaspekt der Adipositas durchaus sinnvoll erscheinen, wenngleich nicht eindeutig zu klären ist, ob Depressionen vermehrt aufgrund von Adipositas entstehen oder ggf. Adipositas auch als eine mögliche Folge von Depressionen zu sehen ist (z.B. „Frustessen“).

\footnotetext{
${ }^{105}$ Vgl. http://www.aerztemagazin.at: DFP-Allgemeinmedizin: Akute Pankreatitis.

${ }^{106} \mathrm{Groß}$ et al. (2006): Online-Publikation unter http://www.egms.de/en/meetings/dgu2006/06dgu1005.shtml.

${ }^{107}$ Siehe z.B. Kalarchian, M. A. (2007), S. 328-334.

${ }^{108} \mathrm{Vgl}$. ebenda.
} 
Schlafstörungen/ Schlafapnoe-Syndrom (ohne Pickwick-Syndrom) (G 47)

Etwa 4\% der Männer und 2\% der Frauen > 40 Jahre sind vom SchlafapnoeSyndrom betroffen. Rund $80 \%$ davon sind als adipös einzustufen. Bei den klinisch gravierenden Formen dieses Krankheitsbildes ist eine Adipositas mit einem BMI > 35 ca. 20 Mal häufiger vertreten als bei Normalgewichtigen. ${ }^{109}$ Teilweise wird bereits davon ausgegangen, dass höhergradige Adipositas auch ohne diagnostiziertes Schlafapnoe- oder Pickwick-Syndrom die Schläfrigkeit am Tage erhöhen kann. Zur Kostenberechnung kommen, in Abhängigkeit von der Studienlage, die Schlafstörungen (v.a. Schlafapnoe) unter ICD 10 G 47 in Frage.

Die interessierenden Einflüsse der Adipositas auf die Ausprägung von Folgeerkrankungen sind nach epidemiologischen Gesichtspunkten zu untersuchen. Um die Stärke des Zusammenhangs zwischen Adipositas und assoziierten Erkrankungen darzustellen, wird das relative Risiko $(R R)$ ermittelt, welches die Verbindung des Krankheitsrisikos mit der Exposition (hier Adipositas) im Vergleich zu einer gesunden Kontrollgruppe $(R R=1)$ ausdrückt. Die letztlich entscheidende Aussage darüber, welcher Anteil der Krankheitshäufigkeit in der Gesamtpopulation dem Risikofaktor Adipositas zuzuschreiben ist, erhält man über den bereits oben vorgestellten bevölkerungsbezogenen beizumessenden Anteil (PAF), der idealerweise alle medizinisch und statistisch relevanten Aspekte berücksichtigt und sich über einen einzigen abgeleiteten Prozentsatz je Krankheit zeigt.

Die für diese Arbeit relevanten beizumessenden Anteile der betrachteten Erkrankungen beziehen sich auf die in vorangegangen Abschnitten bereits mehrfach zitierten, umfassenden Analysen von Schneider/ Schmid des Jahres 2004. ${ }^{110}$ In der dortigen Analyse geht es um die Bestimmung der Kosten der Adipositas in der gesamten Schweiz. Diese Analyse basiert ihrerseits wiederum auf einer umfangreichen Anzahl von anerkannten internationalen Studien und ist mit Blick auf den hier zu behandelnden, vergleichbaren Zusammenhang als gleichzeitig tragfähig wie auch repräsentativ zu bewerten. Insofern werden die darin ermittelten beizumessenden Anteile auch für die Anforderungen dieser Arbeit als geeignet

${ }^{109}$ Vgl. Young, T. et al. (2002), S. 1217-1239. 
angesehen und für alle weiteren diesbezüglichen Berechnungen herangezogen. ${ }^{111}$ Dabei ist zu berücksichtigen, dass für die hier zu bearbeitende Fragestellung lediglich die beizumessenden Anteile für die als adipös klassifizierten Personen mit einem $\mathrm{BMI} \geq 30$ angesetzt werden. Würde man alle Übergewichtigen ( $25 \leq \mathrm{BMI} \leq 30)$ zusätzlich zu den Adipösen berücksichtigen, lägen die Anteile entsprechend höher. Beispielsweise wird im Falle von Diabetes mellitus von einem fast $90 \%$ attributiven Risiko für übergewichtige und adipöse Personen zusammen ausgegangen, das jedoch bei alleiniger Betrachtung adipöser Personen bei "nur" etwa $42 \%$ liegt. Bei Depression dagegen besteht für Übergewichtige kein erhöhtes attributives Risiko, während bei Adipösen das Risiko auf rund 6\% ansteigt. Eine Unterscheidung zwischen den Geschlechtern wurde nicht vorgenommen, da diese differenzierte Sichtweise eine ebenfalls differenzierte Informationsgrundlage der Kosten erfordern würde, die bzgl. aller zu berücksichtigenden Kostenbestandteile nur bedingt vorhanden ist.

Tabelle 12: Bevölkerungsbezogene beizumessende Anteile (PAF) für assoziierte Erkrankungen im Zusammenhang mit Adipositas

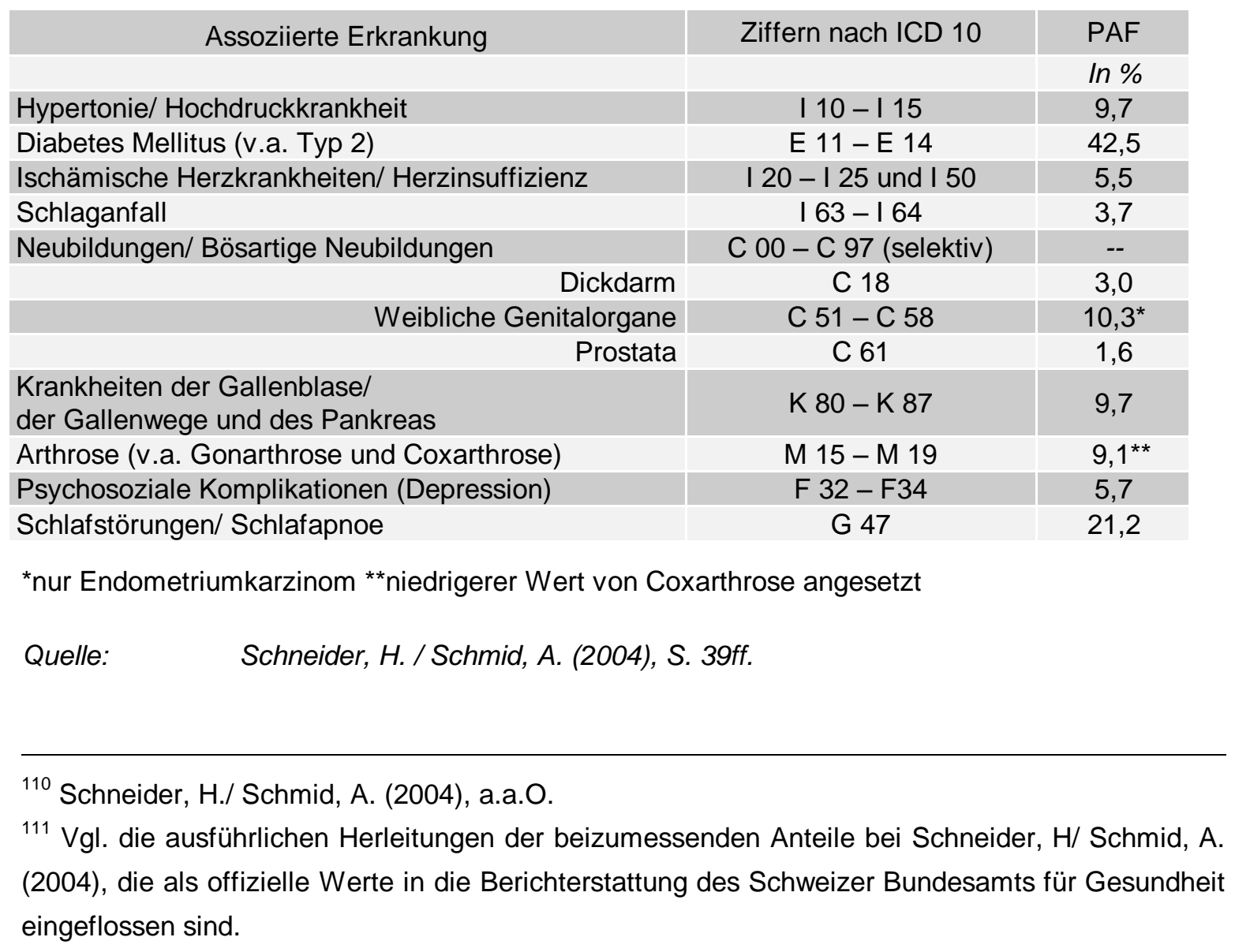


Aus den weiter oben dargelegten Gründen wird zunächst die um die beizumessenden Anteile für Adipositas modifizierte Gesundheitskostenrechnung des Bundes vorangestellt. Dann werden die eigenen Berechnungen auf Grundlage von recherchierten Einzelstudien dargelegt und mit den Ergebnissen auf Grundlage der Ausgangsberechnung des Bundes verglichen.

\section{Gesundheitskostenrechnung des Bundes}

Betrachtet man die Gesundheitskostenrechnung des Bundes, so stehen die direkten Krankheitskosten der distributiven Top-Down-Gesundheitskostenrechnung der wichtigsten assoziierten und auf ICD 10 Nummern bezogenen Krankheiten auf Basis von Tabelle 4 zur Verfügung.

Tabelle 13: Direkte ungewichtete und mit den bevölkerungsbezogenen beizumessenden Anteilen gewichtete Krankheitskosten der Gesundheitskostenrechnung des Bundes (2004) nach ICD 10-Klassifikation

\begin{tabular}{|c|c|c|c|c|}
\hline Assoziierte Erkrankung & $\begin{array}{l}\text { Ziffern nach } \\
\quad \text { ICD } 10\end{array}$ & $\begin{array}{l}\text { Ungewichtete } \\
\text { direkte } \\
\text { Krankheitskosten }\end{array}$ & PAF & $\begin{array}{l}\text { Gewichtete direkte } \\
\text { Krankheitskosten }\end{array}$ \\
\hline & & Jahr 2004 in Mio. $€$ & $\ln \%$ & Jahr 2004 in Mio. $€$ \\
\hline $\begin{array}{l}\text { Hypertonie/ } \\
\text { Hochdruckkrankheit }\end{array}$ & $|10-| 15$ & 8.121 & 9,7 & 788 \\
\hline Diabetes Mellitus (v.a. Typ 2) & E $11-$ E 14 & 5.098 & 42,5 & 2.167 \\
\hline $\begin{array}{l}\text { Ischämische Herzkrankheiten/ } \\
\text { Herzinsuffizienz }\end{array}$ & $\begin{array}{l}\text { I } 20-125 \\
\text { und I } 50\end{array}$ & 6.190 & 5,5 & 340 \\
\hline Schlaganfall & $163-164$ & 2.511 & 3,7 & 93 \\
\hline $\begin{array}{l}\text { Neubildungen/ Bösartige } \\
\text { Neubildungen }\end{array}$ & $\begin{array}{c}\text { C } 00-\text { C } 97 \\
\text { (selektiv) }\end{array}$ & (15.044) & -- & -- \\
\hline Dickdarm & C 18 & 1.197 & 3,0 & 36 \\
\hline Weibliche Genitalorgane & C $51-$ C 58 & 835 & 10,3 & 86 \\
\hline Prostata & C 61 & 1.371 & 1,6 & 22 \\
\hline $\begin{array}{l}\text { Krankheiten der Gallenblase/ } \\
\text { der Gallenwege und des } \\
\text { Pankreas }\end{array}$ & K $80-$ K 87 & 1.835 & 9,7 & 178 \\
\hline $\begin{array}{l}\text { Arthrose (v.a. Gonarthrose und } \\
\text { Coxarthrose) }\end{array}$ & M $15-$ M 19 & 6.769 & 9,1 & 616 \\
\hline $\begin{array}{l}\text { Psychosoziale Komplikationen } \\
\text { (Depression) }\end{array}$ & $F 32-F 34$ & 4.187 & 5,7 & 239 \\
\hline Schlafstörungen/ Schlafapnoe & G 47 & 642 & 21,2 & 135 \\
\hline Summe & -- & 37.385 & -- & 4.699 \\
\hline
\end{tabular}

Quelle:

Eigene Berechnung auf Basis der "Krankheitskosten in Mio. $€$ für Deutschland" der Gesundheitsberichterstattung des Bundes unter http://www.gbe-bund.de. 
Die ungewichteten Kosten der selektierten Einzelerkrankungen (2004) belaufen sich auf insgesamt 37,38 Mrd. €. Die größten Anteile liegen bei Hypertonie (8,1 Mrd. €), Arthrose (6,7 Mrd. €) und Ischämische Herzkrankheiten (6,2 Mrd. €), dicht gefolgt von Kosten für Diabetes mellitus (5,1 Mrd. €). Diese Größenordnungen unterscheiden sich je nach Einzelerkrankung teils wesentlich von den durch Einzelstudien ermittelten Kosten, wie sich im weiteren Verlauf noch zeigen wird. ${ }^{112}$ Nach der PAF-Gewichtung stellt sich die Situation wie folgt dar:

Mit insgesamt rund 4,7 Mrd. $€$ weisen die gewichteten direkten Kosten einen relativen Anteil von 12,5\% an den gesamten direkten Kosten der Gesundheitskostenrechnung auf. Hier wird deutlich, dass Diabetes mellitus mit rund 46\% Kostenanteil klar die teuerste Begleiterkrankung repräsentiert. Hypertonie folgt mit etwa 16\% vor Arthrose (13\%) und Ischämischen Herzkrankheiten (7\%).

\section{Eigene Berechnung auf Grundlage von Einzelstudien}

Eigene Berechnungen sollen nun eine erweiterte Perspektive auf die relevanten direkten Kosten liefern. Der Schwerpunkt wird auf die bestehenden, möglichst aktuellen Einzelstudien der jeweiligen als assoziiert identifizierten Erkrankungen gelegt. Die in den Studien ermittelten direkten Kosten werden dazu mit den beizumessenden Anteilen für Adipositas bewertet und können daraufhin beziffert werden. Es gilt zu bedenken, dass für jede der Studien ggf. unterschiedliche Bezugsgruppen, Zeiträume, methodische Verfahren usw. Anwendung gefunden haben, so dass auch in diesem Zusammenhang, wie bereits im vorangegangenen Kapitel, ein möglichst aktuelles "virtuelles Bezugsjahr" der anteiligen Kosten für Adipositas ermittelt wird, welches unter erschwerten Analysebedingungen als repräsentativ angesehen wird. Wenn keine spezifischen Studien recherchiert werden konnten, wurden die Daten des Statistischen Bundesamts herangezogen, wie im Falle von Hypertonie, Gallenblase/ Pankreas, Bösartige Neubildungen. Die Berechnungen zeigen, dass die gesamten direkten (noch ungewichteten) Krankheitskosten um das 2,1-fache höher eingeschätzt werden als durch die Berechnungen des Statistischen Bundesamtes. Im Falle von Diabetes mellitus stehen im Vergleich rund 3-fach höhere Kosten zu Buche, bei kardiovaskulären

\footnotetext{
${ }^{112}$ Vgl. Tabelle 13 dieser Arbeit und die diesbezüglichen Ausführungen.
} 
Erkrankungen sogar nahezu das 6-fache. Arthrose weist einen 1,5-fach höheren Wert auf, wenngleich hier die Abgrenzungen der Arthrose zu anderen muskuloskelettalen Erkrankungen nur unscharf vorzunehmen ist und der Wert ggf. zu hoch ausfällt. Schlafapnoe wird etwa um das Doppelte höher bewertet.

Tabelle 14: $\quad$ Direkte ungewichtete und mit den bevölkerungsbezogenen beizumessenden Anteilen gewichtete Krankheitskosten (in Mio. €)

\begin{tabular}{|c|c|c|c|c|c|}
\hline Bezugsquellen/ Studien & Jahr & $\begin{array}{l}\text { Erkrankung/ } \\
\text { Erkrankungs- } \\
\text { gruppe }\end{array}$ & $\begin{array}{l}\text { Ungewichtete } \\
\text { direkte Kosten } \\
\text { (in Millionen } € \text { ) }\end{array}$ & $\begin{array}{l}\text { PAF } \\
\text { (in \%) }\end{array}$ & $\begin{array}{c}\text { Gewichtete } \\
\text { direkte Kosten } \\
\text { (in Millionen } € \text { ) }\end{array}$ \\
\hline $\begin{array}{l}\text { Statistisches Budesamt } \\
\text { (2004), Wiesbaden }\end{array}$ & 2004 & Hypertonie & 8.121 & 9,7 & 788 \\
\hline $\begin{array}{l}\text { Köster, I. et al. (2006) } \\
\text { CoDiM Studie }\end{array}$ & 2001 & Diabetes mellitus & 16.032 & 42,5 & 6.814 \\
\hline $\begin{array}{l}\text { Leal, J et al. (2006) } \\
\text { European Society of } \\
\text { Cardiology }\end{array}$ & 2003 & $\begin{array}{l}\text { Kardiovaskuläre } \\
\text { Erkrankungen }\end{array}$ & 34.909 & 5,5 & 1.920 \\
\hline $\begin{array}{l}\text { Kolominsky-Rabas, P. et } \\
\text { al. (2006): The Erlangen } \\
\text { Stroke Project }\end{array}$ & 2006 & Schlaganfall & 3.148 & 3,7 & 116 \\
\hline $\begin{array}{l}\text { Statistisches Bundesamt } \\
\text { (2004), Wiesbaden }\end{array}$ & 2004 & Colonkarzinom & 1.197 & 3,0 & 36 \\
\hline $\begin{array}{l}\text { Statistisches Bundesamt } \\
\text { (2004), Wiesbaden }\end{array}$ & 2004 & $\begin{array}{l}\text { Karzinome der } \\
\text { weiblichen } \\
\text { Geschlechts- } \\
\text { organe }\end{array}$ & 835 & 10,3 & 86 \\
\hline $\begin{array}{l}\text { Statistisches Bundesamt } \\
\text { (2004), Wiesbaden }\end{array}$ & 2004 & $\begin{array}{l}\text { Prostata- } \\
\text { karzinom }\end{array}$ & 1.371 & 1,6 & 22 \\
\hline $\begin{array}{l}\text { Statistisches Bundesamt } \\
\text { (2004), Wiesbaden }\end{array}$ & 2004 & $\begin{array}{l}\text { Gallenblase/ } \\
\text { Pankreas }\end{array}$ & 1.835 & 9,7 & 178 \\
\hline $\begin{array}{l}\text { Dreinhöfer, K. E. (2002) } \\
\text { European Bone and Joint } \\
\text { Health Strategies Projekt } \\
2000-2010\end{array}$ & 2002 & $\begin{array}{l}\text { Arthrose } \\
\text { (muskulosket. } \\
\text { Gelenkerkran- } \\
\text { kungen) }\end{array}$ & 10.610 & 9,1 & 965 \\
\hline $\begin{array}{l}\text { Friemel, S. et al. (2002) } \\
\text { ESEMeD Projekt }\end{array}$ & 2002 & Depression & 1.600 & 5,7 & 91 \\
\hline $\begin{array}{l}\text { Fischer, J./ Raschke, F. } \\
\text { Institut für Rehabilita- } \\
\text { tionsforschung }\end{array}$ & 1997 & $\begin{array}{c}\text { Schlafstörungen } \\
\text { (nur } \\
\text { Schlafapnoe) }\end{array}$ & 1.176 & 21,2 & 249 \\
\hline \multicolumn{3}{|l|}{ Summe } & 80.834 & -- & 11.265 \\
\hline
\end{tabular}

Quelle:

Eigene Berechnung auf Basis unterschiedlicher Studien. 
Lediglich im Fall von Depressionen wurde in der dafür herangezogenen Studie nur rund $40 \%$ des Wertes des Statistischen Bundesamtes ermittelt. Hier könnte u.a. der unklare Zusammenhang zwischen der Entstehung von Depressionen eine Rolle spielen, dass sie entweder als Konsequenz von Adipositas anzusehen sind oder aber ggf. einen begünstigenden Grund für Adipositas darstellen (z.B. Frustessen), was hier nicht zu klären ist.

Aufgrund fehlender Primärquellen wurden insgesamt Kostenanteile von etwa 13,3 Mrd. $€$ aus der Bundesstatistik übernommen (Kosten für Hypertonie, Gallenblase/ Pankreas, Bösartige Neubildungen), was einem Anteil von 16\% (von insgesamt ermittelten 80,8 Mrd. $€$ ) entspricht. Ob diese Angaben ggf. zu geringe Kosten repräsentieren, lässt sich zwar vermuten, jedoch mangels Vergleich nicht klären. Wendet man die beizumessenden Anteile zur Gewichtung der Kosten an, so ergeben sich modifizierte direkte Kosten in Höhe von rd. 11,26 Mrd. €, die auf bestehende Adipositas zurückzuführen sind. Durch die Gewichtung erhöht sich die Abweichung zwischen dem Wert des Statistischen Bundesamts (vgl. Tabelle 13) auf das 2,4-fache. Dabei wird in erster Linie dem hohen Einfluss von Adipositas auf Diabetes mellitus (Primärerkrankung) Rechnung getragen, der jetzt mit rund 6,8 Mrd. $€$ zu Buche steht, im Vergleich zu etwa 2,1 Mrd. $€$ in der Gesundheitskostenrechnung des Bundes. Auch der Anteil der ischämischen Herzkrankheiten von 1,9 Mrd. $€$ wird im Gegensatz zu den 340 Mio. $€$ des Bundes wesentlich aufgewertet. Die Kosten für Arthrose im Rahmen muskuloskelettaler Erkrankungen betragen nach eigenen Berechnungen 965 Mio. $€ \mathrm{im}$ Vergleich zu 616 Mio. $€$ in der offiziellen Statistik.

Da mit den direkten Kosten von Adipositas selbst und den beizumessenden Anteilen der assoziierten Erkrankungen noch nicht das gesamte Ausmaß der Kostenbelastung durch Adipositas in Deutschland beschrieben ist, werden im Anschluss mit den indirekten Kosten die noch fehlenden Anteile berechnet.

\subsubsection{Indirekte Kosten}

Es wurde ausführlich gezeigt, dass medizinisch und hinsichtlich entstehender direkter Kosten, von einer durch Adipositas erzeugten, gewichtigen Kausalität für 
die Entstehung oder Begünstigung bestimmter assoziierter Erkrankungen ausgegangen werden muss. Zur Berechnung der indirekten Kosten ist dieser Zusammenhang von besonderer Bedeutung, da die entscheidenden Kriterien Arbeitsausfall, Invalidität und vorzeitiger Tod von Betroffenen im erwerbsfähigen Alter primär erst als Konsequenz dieser assoziierten Erkrankungen eine Rolle spielen und nicht etwa allein nur durch das Vorhandensein von Adipositas per se. ${ }^{113}$ Der Beitrag indirekter Kosten durch Adipositas wird zwar mitberücksichtigt und fließt konsequenterweise mit einem beizumessenden Anteil von $100 \%$ in die Berechnung ein, repräsentiert aber nur einen kleineren Teil der gesamten indirekten Kosten aller Folge- und Begleiterkrankungen. Setzt man dieses Verständnis für die weiteren Berechnungen voraus, so wird sich zeigen, dass diese teils nur mit höherem Aufwand durchzuführen sind, da die offizielle Statistik die erforderlichen Daten nicht in benötigtem Umfang bzw. in der benötigten Detailtiefe vorhält. Zudem wurde das Jahr 2003 als Bezugsjahr ausgewählt, da hierfür die benötigten „Hilfsdaten“ weitestgehend vollständig zur Verfügung stehen, was für spätere Jahre noch nicht gilt. Die Berechnungen der indirekten Kosten folgen im Wesentlichen den methodischen Grundsätzen, die bereits im Rahmen der Studie über „Ernährungsabhängige Krankheiten und ihre Kosten“ aus dem Jahr 1992 angewendet wurden. ${ }^{114}$ Die Einzelschritte sind wie folgt durchzuführen:

- Identifikation der Anzahl von Jahren an Arbeitsunfähigkeit, Invalidität und vorzeitigem Tod für jede der assoziierten Erkrankungen, gegliedert nach den ICD 10 Ziffern soweit nach Einzeldiagnosen verfügbar

- Anwendung einer Bruttoeinkommensrechnung unselbständiger Erwerbstätiger unter Zuhilfenahme der Humankapital-Methode ( $\mathrm{vgl}$. Abschnitt 2.2.2)

- Diskontierung der inzidenzbasierten Teilergebnisse (Invalidität, Mortalität) auf Gegenwartswerte mit üblichen Diskontierungssätzen (4\% und 6\%)

- Gewichtung der Kosten der Einzelerkrankungen mit den relevanten beizumessenden Anteilen

\footnotetext{
${ }^{113}$ Schneider, H. et al (2004), a.a.O. S. 7 und S. 47.

${ }^{114}$ Kohlmeier, L. et. al (1993), a.a.O.
} 
Aus Gründen der Übersichtlichkeit wurden einige Tabellen, Herleitungen und Berechnungsschritte der indirekten Kostenbestandteile in den Anhang dieser Arbeit verlegt. Im laufenden Text wird sich im Wesentlichen auf Tabellen/ Schaubilder beschränkt, welche die aggregierten Endergebnisse aus den Einzelberechnungen veranschaulichen.

\section{Arbeitsunfähigkeit}

Zur Berechnung der Arbeitsunfähigkeit wird eine Prävalenzbetrachtung durchgeführt. Hierzu ist es erforderlich, die Arbeitsunfähigkeitstage aller Erwerbstätigen eines Betrachtungsjahres zu ermitteln/ hochzurechnen und daraufhin die für die Volkswirtschaft verlorenen Produktivzeiten monetär zu bewerten. Mit dieser Zielsetzung werden die Ausfallzeiten mit dem durchschnittlichen Bruttoeinkommen aus unselbständiger Vollzeitarbeit bewertet, das vom Bundesministerium für Arbeit und Soziales mit $40.521 €$ (Männer) $€$ und $30.576 €$ (Frauen) beziffert wird. ${ }^{115}$

Die Bundesanstalt für Arbeitsschutz und Arbeitsmedizin hat für das Jahr 2003 die Anzahl von 31,49 Mio. Arbeitsunfähigkeitstagen (AU-Tagen) für alle Pflichtversicherten und freiwillig Versicherten der GKV in Deutschland ermittelt. ${ }^{116}$ Durch Hochrechnung auf alle 34,145 Mio. Arbeitnehmer ergeben sich bei einem durchschnittlichen Wert von 13,7 AU-Tagen pro Kopf 467,79 Mio. Tage, an denen aus Krankheitsgründen nicht gearbeitet wurde. Zu berücksichtigen ist, dass lediglich die Fehlzeiten erfasst werden können, die über Arbeitsunfähigkeitsbescheinigungen an die Krankenkassen einzureichen sind und somit i.d.R. über einer Fehlzeit von 3 Tagen liegen. Die Arbeitsausfälle unter 3 Tagen betragen im Rahmen o.g. Schätzungen der Bundesanstalt für Arbeit und Soziales etwa 6\% aller AU-Tage.

\footnotetext{
${ }^{115} \mathrm{Vgl}$. Bundesministerium für Arbeit und Soziales (2005). Es finden sich je nach Branchenabgrenzung und Einbeziehung von Voll- und/ oder Teilzeit unterschiedliche Angaben. I.d.R. werden der Handel, das produzierende Gewerbe sowie das Kredit- und Versicherungsgewerbe einbezogen.

${ }^{116}$ Bundesanstalt für Arbeit uns Soziales (o. J.): Volkswirtschaftliche Kosten für Arbeitsunfähigkeit. 2003. www.baua.de.
} 
Tabelle 15: $\quad$ Arbeitsunfähigkeitstage aller AOK-pflichtversicherten Arbeitnehmer 2003 ungewichtet und gewichtet mit den beizumessenden Anteilen (PAF)

\begin{tabular}{|l|r|r|r|r|r|}
\hline & \multicolumn{1}{|c|}{ Männer } & \multicolumn{1}{|c|}{ Frauen } & \multicolumn{1}{|l}{ PAF } & Männer & Frauen) \\
\hline & ungewichtet & Ungewichtet & In \% & $\begin{array}{c}\text { mit PAF } \\
\text { gewichtet }\end{array}$ & $\begin{array}{c}\text { mit PAF } \\
\text { gewichtet }\end{array}$ \\
\hline Hypertonie & 866.846 & 579.788 & 9,7 & 84.084 & 56.239 \\
\hline Diabetes mellitus & 381.145 & 118.105 & 42,5 & 161.987 & 50.195 \\
\hline KHK & 2.094 .038 & 448.385 & 5,5 & 115.172 & 24.661 \\
\hline Schlaganfall & 447.530 & 166.987 & 3,7 & 16.559 & 6.179 \\
\hline Colon-CA & 74.313 & 53.239 & 3,0 & 2.229 & 1.597 \\
\hline CA q Geschlechtsorgane & 0 & 133.233 & 10,3 & 0 & 13.723 \\
\hline Prostata-CA & 148.196 & 0 & 1,6 & 2.371 & 0 \\
\hline Gallenblase/ Pankreas & 443.913 & 403.952 & 9,7 & 43.060 & 39.183 \\
\hline Arthrose & 1.978 .843 & 1.322 .294 & 9,1 & 180.075 & 120.329 \\
\hline Depression & 1.498 .455 & 2.324 .289 & 5,7 & 85.412 & 132.484 \\
\hline Schlafapnoe & 134.345 & 62.345 & 21,2 & 28.481 & 13.217 \\
\hline Adipositas & 29.106 & 41.451 & 100 & 29.106 & 41.451 \\
\hline Summe & $\mathbf{8 . 0 9 6 . 7 3 0}$ & $\mathbf{5 . 6 5 4 . 0 6 8}$ & -- & $\mathbf{7 4 8 . 5 3 5}$ & $\mathbf{4 9 9 . 2 5 9}$ \\
\hline
\end{tabular}

Quelle:

Eigene Berechnungen auf Basis von: AOK-Krankheitsartenstatistik

Für die hier behandelte Fragestellung spielen nur die Arbeitsausfälle eine Rolle, die durch ICD 10-Erkrankungen entstanden sind, welche in enger Abhängigkeit zur Adipositas gesehen und als solche definiert werden. Zur Ermittlung dieser Daten konnte die AOK-Krankheitsartenstatistik des Jahres 2003 herangezogen werden. ${ }^{117}$ Darin werden sämtliche AU-Fälle und -tage des Bezugsjahres für alle AOK-Mitglieder erfasst und gemäß ICD 10-Klassifizierung aufgelistet. Zusammen mit den beizumessenden Anteilen der AU-Tage wird eine gewichtete und ungewichtete Sicht auf die Daten dargestellt.

Es zeigt sich, dass die Arbeitsausfälle bei Männern mit 748.535 deutlich höher liegen als die 499.259 Tage bei den Frauen. Bezieht man diese Zahlen auf die noch ungewichteten AU-Tage (also ohne Berücksichtigung der beizumessenden Anteile) der Männer in Höhe von 8.096.730 und der Frauen in Höhe von 5.654.086 so ergeben sich relative Anteile von $9,24 \%$ bzw. $8,83 \%$, die durch Arbeitsausfälle aufgrund des Bestehens von Adipositas bedingt sind.

${ }^{117}$ AOK-Bundesverband: Krankheitsartenstatistik 2003. 
Die so ermittelten AU-Daten je Einzelerkrankungen für Arbeitsunfähigkeit können über einige Berechnungszwischenschritte (vgl. Anhang) auf alle GKV-Pflichtversicherten hochgerechnet werden. Dazu ist es erforderlich, die Mitgliederverteilungsstruktur der Pflichtversicherten auf die gesetzlichen Krankenkassen in Deutschland heranzuziehen, die aus der GKV-Statistik des Bundesministeriums für Gesundheit und soziale Sicherung zu erschließen ist. ${ }^{118} \mathrm{Im}$ Zuge der Berechnungen werden die Zahlen von Arbeitslosen und Rentnern herausgerechnet, da diese Personengruppen nicht aktiv am Arbeitsleben teilnehmen und demnach bei Krankheit auch keinen Arbeitszeitenverlust erzeugen. Privatversicherte bleiben bei dieser Betrachtung gänzlich unberücksichtigt, da erforderliche Informationen hierzu aus Wettbewerbsgründen der privaten Kassen nicht zugänglich sind.

Es zeigt sich bei Betrachtung der Mitgliederverteilungsrelationen, dass AOK, BKK und Ersatzkassen zusammen bei den pflichtversicherten Männern rund $85 \%$ und bei den Frauen über 90\% ausmachen, während die übrigen Kassen eine untergeordnete Rolle spielen (vgl. Anhang). Unterstellt man nun im Weiteren vereinfachend, dass die Versicherten aller Kassen gleiche Alters- und Risikostrukturen aufweisen und zudem, dass die aus der AOK-Krankheitsartenstatistik berechneten Relationen der AU-Tage bzgl. der relevanten Erkrankungen in vergleichbarer Weise bei den anderen GKV vorherrschen, so lassen sich die gesamten AU-Tage aufgrund von Adipositas auf alle Pflichtversicherten in Deutschland hochrechnen:

${ }^{118}$ Bundesministerium für Gesundheit und soziale Sicherung; Ergebnisse der GKV-Statistik KM 1 (Stand Januar 2004). 


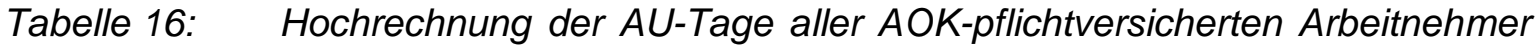
2003 auf die restlichen GKV in Deutschland (Basis: AOK-Zahlen)

\begin{tabular}{|c|c|c|c|c|c|c|c|c|c|}
\hline & Summe & $\mathrm{AOK}^{*}$ & BKK & InnungsKK & $\begin{array}{l}\text { Land- } \\
\text { wirt. } \\
\text { KK }\end{array}$ & $\begin{array}{l}\text { See } \\
\text { KK }\end{array}$ & $\begin{array}{l}\text { Bundes- } \\
\text { knapp- } \\
\text { schaft }\end{array}$ & $\begin{array}{l}\text { Ersatzk.A } \\
\text { rbeiter }\end{array}$ & $\begin{array}{c}\text { Ersatzk. } \\
\text { Ange-stellte }\end{array}$ \\
\hline $\begin{array}{l}\text { Versicherte } \\
\text { Männer, Angaben } \\
\text { in \% }\end{array}$ & $100,00 \%$ & $35,89 \%$ & $27,09 \%$ & $9,41 \%$ & $1,60 \%$ & $0,12 \%$ & $1,13 \%$ & $2,94 \%$ & $21,81 \%$ \\
\hline $\begin{array}{l}\text { Hochgerechnete } \\
\text { AU-Tage Männer }\end{array}$ & 2.085 .602 & $748.535^{\star}$ & 564.965 & 196.327 & 33.397 & 2.569 & 23.644 & 61.311 & 454.854 \\
\hline $\begin{array}{l}\text { Versicherte } \\
\text { Frauen, Angaben } \\
\text { in \% }\end{array}$ & $100,00 \%$ & $27,64 \%$ & $24,60 \%$ & $4,92 \%$ & $0,22 \%$ & $0,02 \%$ & $0,31 \%$ & $1,77 \%$ & $40,53 \%$ \\
\hline $\begin{array}{l}\text { Hochgerechnete } \\
\text { AU-Tage Frauen }\end{array}$ & 1.806 .290 & $499.259^{*}$ & 444.350 & 88.929 & 3.913 & 331 & 5.590 & 31.898 & 732.020 \\
\hline $\begin{array}{l}\text { Gesamt Männer } \\
\text { und Frauen }\end{array}$ & 3.891 .892 & $1.247 .794^{\star}$ & 1.009 .315 & 285.256 & 37.310 & 2.899 & 29.234 & 93.208 & 1.186 .874 \\
\hline
\end{tabular}

Quelle:

Eigene Berechnungen auf Basis von: AOK-Krankheitsartenstatistik sowie KM 1 Statistik des Bundesministeriums für Gesundheit und Soziales

Um die damit verbundenen indirekten Kosten zu erhalten, ist als nächster Schritt eine Umrechnung der AU-Tage in AU-Jahre erforderlich. Hier wird die offiziell anerkannte Pauschalregel der deutschen Finanzbehörden von 240 Arbeitstagen pro Jahr herangezogen. Die ermittelten Jahre sind in einem weiteren Schritt mit dem durchschnittlichen Bruttojahreseinkommen zu bewerten und führen schließlich zu nachfolgendem Ergebnis. ${ }^{119}$ Eine Diskontierung ist an dieser Stelle nicht notwendig, da AU-Tage befristet sind und lediglich über eine Betrachtungsperiode anfallen. Die ökonomische Belastung aufgrund von dauerhafter Arbeitsunfähigkeit (i.S.d. Frühberentung) wird über die indirekten Kosten für Invalidität erfasst.

Tabelle 17: Indirekte Kosten für Arbeitsunfähigkeit nach Geschlecht

\begin{tabular}{|c|c|c|}
\hline & Männer & Frauen \\
\hline AU-Jahre (Gesamttage : 240) & 8.690 & 7.526 \\
\hline $\begin{array}{c}\text { Durchschnittliches Bruttoeinkommen } \\
\text { pro Jahr in } €\end{array}$ & 40.521 & 30.576 \\
\hline $\begin{array}{c}\text { AU-Tage x Bruttoeinkommen in } € \\
\text { Summe Männer und Frauen in } €\end{array}$ & $\mathbf{3 5 2 . 1 2 7 . 4 9 0}$ & $\mathbf{2 3 0 . 1 1 4 . 9 7 6}$ \\
\hline
\end{tabular}

Quelle: $\quad$ Eigene Berechnungen auf Basis aller verwendeten Quellen. 
Die gesamten indirekten Kosten für Arbeitsunfähigkeit aufgrund von Adipositas belaufen sich nach diesen Berechnungen auf über 582 Mio. $€$. Mit Blick auf die differenzierte Verteilung der Einzelerkrankungen sind bei den Männern die meisten AU-Tage und demnach auch die höchsten indirekten Kosten für Arthrose (24\%) mit 84,5 Mio. €, Diabetes (22\%) mit 77,4 Mio. € und Ischämische Herzerkrankungen (15\%) mit 52,8 Mio. € nachzuweisen. Dies macht zusammen den Betrag von 214,7 Mio. € oder rd. 36,8\% der gesamten indirekten Kosten für Arbeitsunfähigkeit beider Geschlechter aus. Bei den Frauen dominieren die Erkrankungen Depression (26\%) mit 59,8 Mio. € und Arthrose (24\%) mit 55,2 Mio. $€$, gefolgt von Hypertonie (11\%) 25,3 Mio. $€$. Sie sind zusammen für rund 141 Mio. $€$ verantwortlich und repräsentieren einen Anteil von rund $24,1 \%$ der in diesem Zusammenhang betrachteten gesamten Kosten beider Geschlechter. Weitere Einzelansichten auf die Kosten für Arbeitsunfähigkeit finden sich im Anhang.

\section{$\underline{\text { Invalidität }}$}

Als nächstes gilt die Aufmerksamkeit den indirekten Kosten aufgrund von frühzeitiger Erwerbsminderung oder -unfähigkeit. Die Invaliditätsfälle für das Jahr 2003 wurden über das Referat für Statistische Analysen und Methoden der Deutschen Rentenversicherung abgefragt. Diese Rohdaten der gesetzlichen Rentenversicherung liegen für alle ICD 10-Erkankungen vor und sind nach Geschlecht und Altersgruppen gegliedert. Die Altersgruppeneinteilung beginnt allerdings erst mit dem Intervall „bis 39“ und differenziert nicht zwischen jüngeren Altersgruppen. Die Altersgruppe „60 und höher" legt keine Obergrenze bis 65 Jahre fest. Diese und weitere Datenaspekte erfordern eine Reihe von Annahmen für weitere Berechnungen, die detailliert bei den relevanten Tabellen im Anhang angeführt werden.

Die dargestellten Fallzahlen sind bereits mit der sog. Überlebensquote der einzelnen Altersgruppen gewichtet, die anhand der unspezifischen Sterbestatistik (Absterbeordnung) des Bundes ermittelt wurde und berücksichtigt, dass nicht alle

\footnotetext{
119 Durchschnittliche Bruttomonatsverdienste vollzeitbeschäftigter Arbeitnehmer/-innen in Deutschland im Juli 2005, hochgerechnet auf das Kalenderjahr, unter www.destatis.de.
} 
Personen der jeweiligen Altersgruppen ihre Restlebenserwartung erreichen. ${ }^{120}$ Auf Basis dieser „korrigierten“ Fallzahlen ergeben sich die durch Invalidität verlorenen Erwerbstätigkeitsjahre für die relevanten ICD 10-Erkrankungen wie folgt:

Tabelle 18: $\quad$ Verlorene Erwerbsjahre durch Invalidität nach Geschlecht getrennt, alle Altersgruppen beginnend mit Personen "bis 39" bis "60 und höher" (ungewichtet und gewichtet mit den beizumessenden Anteilen PAF)

\begin{tabular}{|c|c|c|c|c|c|}
\hline Verlorene Erwerbsjahre & Männer & Frauen & PAF & Männer & Frauen \\
\hline Alle Altersgruppen & Ungewichtet & Ungewichtet & $\ln \%$ & $\begin{array}{l}\text { mit PAF } \\
\text { gewichtet }\end{array}$ & $\begin{array}{l}\text { mit PAF } \\
\text { gewichtet }\end{array}$ \\
\hline Hypertonie & $12.447,0$ & $6.718,1$ & 9,7 & $1.207,4$ & 651,7 \\
\hline Diabetes mellitus & $21.089,2$ & $8.287,1$ & 42,5 & $8.962,9$ & $3.522,0$ \\
\hline KHK & $49.217,6$ & $9.918,3$ & 5,5 & $2.707,0$ & 545,5 \\
\hline Schlaganfall & $31.961,4$ & $17.197,2$ & 3,7 & $1.182,6$ & 636,3 \\
\hline Colon CA & $6.690,1$ & $5.716,3$ & 3,0 & 200,7 & 171,5 \\
\hline CA $\&$ Geschlechtsorgane & 0,0 & $20.719,7$ & 10,3 & 0,0 & $2.134,1$ \\
\hline Prostata CA & $6.023,1$ & 0,0 & 1,6 & 96,4 & 0,0 \\
\hline Gallenblase/ Pankreas & $6.085,4$ & $1.637,9$ & 9,7 & 590,3 & 158,9 \\
\hline Arthrose & $42.913,9$ & $34.667,5$ & 9,1 & $3.905,2$ & $3.154,7$ \\
\hline Depression & $63.563,8$ & $123.430,8$ & 5,7 & $3.623,1$ & $7.035,6$ \\
\hline Schlafapnoe & $1.073,7$ & 423,0 & 21,2 & 227,6 & 89,7 \\
\hline Adipositas & $4.230,5$ & $3.927,2$ & 100 & $4.230,5$ & $3.927,2$ \\
\hline Summe & 245.295,7 & $232.643,1$ & -- & $26.933,6$ & $22.027,1$ \\
\hline
\end{tabular}

Quelle: $\quad$ Eigene Berechnungen auf Basis der angegebenen Quellen (gerundet)

Mit 245.295 Jahren bei den Männern und einem etwas geringeren Wert von 232.643 Jahren bei den Frauen schlagen beträchtliche ungewichtete Ausfallgrößen zu Buche. Während bei beiden Geschlechtern Depressionen mit 26\% bei den Männern und über $50 \%$ bei den Frauen die größte Rolle spielen, sind die weiteren Invaliditätsgründe bei den Männern KHK (20\%) und Arthrose (17\%) und bei den Frauen Arthrose (15\%) und Karzinome der Geschlechtsorgane (9\%). Mit der Gewichtung durch die beizumessenden Anteile für Adipositas verschieben sich die Schwerpunkte bei den Männern hin zu Diabetes mellitus, der mit rund 9.000 verlorenen Erwerbsjahren (33\%) den größten Beitrag leistet, gefolgt von Adipositas als Einzeldiagnose (15\%) und Arthrose (14\%). Bei den Frauen liegt die Depression als wichtigster Invaliditätsgrund mit 32\% oder rund 7.000 Ausfalljahren klar an erster Stelle, gefolgt von der Adipositas (18\%) und Diabetes

\footnotetext{
${ }^{120}$ Vgl. Statistisches Jahrbuch 2006 für die Bundesrepublik Deutschland: Sterbetafeln 2002/ 2004.
} 
mellitus (16\%). Durch einen beizumessenden Anteil von 100\% erhält die Diagnose Adipositas bei dieser Betrachtung der indirekten Kosten, im Vergleich zu den direkten Kosten, einen deutlich höheren Stellenwert.

Zielt man auf die Ermittlung der indirekten Kosten ab, so lassen sich die identifizierten verlorenen Erwerbsjahre monetär bewerten. Auch hier kann zur besseren Vergleichbarkeit eine Unterscheidung zwischen den monetär bewerteten aber zunächst noch ungewichteten und den durch die beizumessenden Anteile gewichteten Jahren erfolgen.

Tabelle 19: Indirekte Kosten durch Invalidität (beide Geschlechter) ungewichtet und mit PAF gewichtet bei Diskontierungsraten 4\% und 6\%

\begin{tabular}{|c|c|c|c|c|c|}
\hline & $\begin{array}{c}4 \% \\
\begin{array}{c}\text { Diskontierungs- } \\
\text { rate }\end{array}\end{array}$ & $\begin{array}{c}6 \% \\
\begin{array}{c}\text { Diskontierungs- } \\
\text { rate }\end{array}\end{array}$ & PAF & $\begin{array}{l}4 \% \\
\text { Diskontierungs- } \\
\text { rate }\end{array}$ & $\begin{array}{c}6 \% \\
\text { Diskontierungs- } \\
\text { rate }\end{array}$ \\
\hline & Ungewichtet & Ungewichtet & & Gewichtet & Gewichtet \\
\hline Erkrankung & $\begin{array}{c}\text { Beide } \\
\text { Geschlechter } \\
\text { In } €\end{array}$ & $\begin{array}{c}\text { Beide } \\
\text { Geschlechter } \\
\text { In } €\end{array}$ & $\ln \%$ & $\begin{array}{c}\text { Beide } \\
\text { Geschlechter } \\
\text { In } €\end{array}$ & $\begin{array}{c}\text { Beide } \\
\text { Geschlechter } \\
\text { In } €\end{array}$ \\
\hline Hypertonie & $323.548 .039,3$ & $259.276 .109,3$ & 9,7 & $31.384 .159,8$ & $25.149 .782,6$ \\
\hline Diabetes mellitus & $480.720 .780,0$ & $377.959 .217,2$ & 42,5 & 204.306.331,5 & $160.632 .667,3$ \\
\hline KHK & $986.596 .264,5$ & $784.219 .333,1$ & 5,5 & $54.262 .794,5$ & 43.132.063,3 \\
\hline Schlaganfall & $757.643 .993,3$ & $584.101 .178,8$ & 3,7 & $28.032 .827,8$ & $21.611 .743,6$ \\
\hline Colon CA & $195.322 .710,9$ & $151.541 .834,3$ & 3,0 & $5.859 .681,3$ & $4.546 .255,0$ \\
\hline CA + Geschlechtsorgane & $286.729 .276,2$ & $210.678 .606,7$ & 10,3 & $29.533 .115,5$ & $21.699 .896,5$ \\
\hline Prostata CA & $112.321 .581,9$ & $93.526 .824,9$ & 1,6 & $1.797 .145,3$ & $1.496 .429,2$ \\
\hline Gallenblase/ Pankreas & $99.175 .306,5$ & $70.168 .210,4$ & 9,7 & $9.620 .004,7$ & $6.806 .316,4$ \\
\hline Arthrose & $1.291 .943 .741,0$ & $1.027 .204 .908,4$ & 9,1 & $117.566 .880,4$ & $93.475 .646,7$ \\
\hline Depression & $2.711 .100 .521,2$ & $2.031 .652 .964,2$ & 5,7 & $154.532 .729,7$ & $115.804 .219,0$ \\
\hline Schlafapnoe & $21.733 .244,0$ & $16.331 .835,0$ & 21,2 & $4.607 .447,7$ & $3.462 .349,0$ \\
\hline Adipositas & $119.240 .507,4$ & $89.495 .441,1$ & 100 & $119.240 .507,4$ & $89.495 .441,1$ \\
\hline Summe & 7.386.075.966,4 & $5.696 .156 .463,4$ & -- & $760.743 .625,7$ & $587.312 .809,7$ \\
\hline
\end{tabular}

Quelle: $\quad$ Eigene Berechnungen auf Basis der angegebenen Quellen (gerundet).

Prinzipiell wird dabei ein vergleichbares Vorgehen gewählt wie im Falle der indirekten Kosten für Mortalität, welches schrittweise und über Zwischenergebnisse zu den letztlich interessierenden Kosten führt. Anders als in der Erhebung der indirekten Kosten für Arbeitsunfähigkeit im Sinne eines Prävalenzansatzes ist hier eine Zukunftssicht (Inzidenzansatz) zu berücksichtigen. Da Zeitreihen über 
teils beträchtliche Lebensabschnitte berücksichtigt werden, wird ein einheitliches durchschnittliches Bruttoeinkommen aus Voll- und Teilzeit angesetzt.

Wird die Invalidität eines Patienten anerkannt, so ist er dies i.d.R. bis zum Ende seines Erwerbslebens und darüber hinaus. Dies bedeutet, dass ab dem Feststellungszeitpunkt zukünftige Erwerbsjahre verloren gehen, die bereits gegenwärtig bewertet werden müssen. Durch eine übliche Diskontierung (mit $4 \%$ bzw. $6 \%$ ) dieser Zukunftswerte wird dieser Anforderung Folge geleistet. ${ }^{121}$ Auf eine Beibehaltung der Unterscheidung beider Geschlechter soll an dieser Stelle aus Komplexitätsgründen verzichtet werden. Im Anhang sind hierzu weitere Tabellen hinterlegt.

Die Umfänge der ungewichteten Kostenanteile von etwa 7,3 Mrd. € (bei 4\% Diskontierung) und 5,7 Mrd. $€$ (bei $6 \%$ Diskontierung) verändern sich durch die beizumessenden Anteile auf rund 760 Mio. $€$ bzw. 587 Mio. $€$, was relativen Anteilen von rund 10,3\% entspricht. Durch die adipositasspezifische Gewichtung rückt erneut die Kostenbelastung aufgrund von Diabetes mellitus in den Vordergrund, die einen Anteil von 27\% repräsentiert. Die Depression spielt als Invaliditätsgrund (trotz nicht eindeutig geklärtem Adipositas-Zusammenhang) eine nach wie vor maßgebliche Rolle und folgt mit ca. 154 Mio. $€$ bzw. 116 Mio. $€(20 \%)$ auf Rang zwei. Arthrose ist nicht nur in der Arbeitsunfähigkeitsstatistik ein wesentlicher Kostentreiber, auch im Rahmen der Langzeitausfälle trägt sie mit 117 Mio. $€$ bzw. 93 Mio. $€$ einen beträchtlichen Kostenanteil (etwa 15\%) bei. Bemerkenswert ist schließlich die Tatsache, dass die Diagnose von Adipositas selbst ebenfalls eine gravierende Kostendeterminante bei Invalidität darstellt. Mit 119 Mio. $€$ bzw. 89 Mio. $€$ fällt sie in etwa gleicher Weise ins Gewicht (rund 15\%) wie Arthrose. Dies ist eine Erkenntnis, die sich nur bedingt mit den Berechnungen der vergleichsweise geringen direkten Kosten von Adipositas per se (ohne Begleiterkrankungen) vereinbaren lässt, was - wie erwähnt - v.a. mit der nur spärlichen Datenbasis dieses Untersuchungsbereichs in Verbindung zu bringen ist.

${ }^{121}$ Vgl. z.B. Kohlmeier, L.. et al. (1993) und die Ausführungen im Diskussionsteil dieser Arbeit. 


\section{Mortalität}

Die Ermittlung der indirekten Kosten aufgrund von Mortalität ist nur mit Hilfe einiger Berechnungsumwege vorzunehmen. Zunächst müssen die Sterbefälle für die relevanten ICD 10-Erkrankungen ermittelt werden. Aufgrund der im Rahmen des Humankapital-Ansatzes vorgesehenen Diskontierung der zu ermittelten Kosten ist - wie im Falle der Invalidität - die Darstellung nach Altersgruppen erforderlich. Nach Extrapolation der Sterbefälle auf die Gesamtbevölkerung und der Berücksichtigung von genereller Überlebenswahrscheinlichkeit und Erwerbsquote je Altersgruppe können die verlorenen Erwerbsjahre beziffert werden, die durch Mortalität aufgrund der assoziierten Erkrankungen entstehen.

Tabelle 20: Verlorene Erwerbsjahre durch Mortalität nach Geschlecht getrennt, alle Altersgruppen zwischen 15 und 65 Jahren (ungewichtet und gewichtet)

\begin{tabular}{|c|c|c|c|c|c|}
\hline Verlorene Erwerbsjahre & Männer & Frauen & PAF & Männer & Frauen \\
\hline Alle Altersgruppen & ungewichtet & ungewichtet & $\ln \%$ & $\begin{array}{c}\text { mit PAF } \\
\text { gewichtet }\end{array}$ & $\begin{array}{c}\text { mit PAF } \\
\text { gewichtet }\end{array}$ \\
\hline Hypertonie & $6.507,5$ & $2.415,0$ & 9,7 & 631,2 & 234,3 \\
\hline Diabetes mellitus & $11.297,5$ & $3.930,0$ & 42,5 & $4.801,4$ & $1.670,3$ \\
\hline KHK & $102.282,5$ & $22.175,0$ & 5,5 & $5.625,5$ & $1.219,6$ \\
\hline Schlaganfall & $7.915,0$ & $3.435,0$ & 3,7 & 292,9 & 127,1 \\
\hline Colon CA & $10.992,5$ & $7.345,0$ & 3,0 & 329,8 & 220,4 \\
\hline CA $q$ Geschlechtsorgane & 0,0 & $19.832,5$ & 10,3 & 0,0 & $2.042,7$ \\
\hline Prostata CA & $2.997,5$ & 0,0 & 1,6 & 48,0 & 0,0 \\
\hline Gallenblase/ Pankreas & $5.442,5$ & $2.665,0$ & 9,7 & 527,9 & 258,5 \\
\hline Arthrose & 0,0 & 2,5 & 9,1 & 0,0 & 0,2 \\
\hline Depression & 0,0 & 32,5 & 5,7 & 0,0 & 1,9 \\
\hline Schlafapnoe & 85,0 & 0,0 & 21,2 & 18,0 & 0,0 \\
\hline Adipositas & $2.545,0$ & $1.442,5$ & 100 & $2.545,0$ & $1.442,5$ \\
\hline Summe & $150.065,0$ & $63.275,0$ & -- & $14.819,7$ & $7.217,4$ \\
\hline
\end{tabular}

Quelle: $\quad$ Eigene Berechnungen auf Basis der angegebenen Quellen (gerundet)

Die Berechnungen der verlorenen Erwerbsjahre basieren, wie im vorangegangenen Abschnitt auch, auf vereinfachenden Annahmen, dass sich die Individuen nur nach Alter und Geschlecht unterscheiden und darüber hinaus beispielsweise keine spezifischen Risikoprofile aufweisen. Zudem muss vereinfachend von einer Konstanz der Verhältnisse (Lohn, Beschäftigung, Überlebenswahrscheinlichkeit usw.) ausgegangen werden. Da sich die verlorenen Erwerbsjahre auf Altersgrup- 
pen von je 5 Jahresabschnitten beziehen, wird unterstellt, dass innerhalb jeder Altersgruppe eine Gleichverteilung von Mortalitätsereignissen vorliegt. So gilt z.B. für den Tod eines Menschen aus der Altersgruppe 30-35, dass für inn und alle anderen aus dieser Gruppe im Mittel 32,5 Lebensjahre (bis zum 65. Lebensjahr) verloren gehen.

Grundsätzlich liegt die Erwerbstätigenquote bei Männern deutlich höher als bei Frauen, so dass Männer in der Statistik in größerem Umfang repräsentiert werden. Die etwa 150.000 verlorenen Erwerbsjahre bei den Männern und rund 63.000 bei den Frauen relativieren sich durch die Gewichtung auf 14.819 (9,8\%) bzw. 7.217 (11,4\%). Bei den Männern fallen die (jetzt gewichteten) Todesfälle aufgrund von assoziierten Herzerkrankungen mit einem Anteil von 38\% am stärksten ins Gewicht, gefolgt von Diabetes mellitus (32\%) und Adipositas (17\%). Bei den Frauen stehen die bösartigen Neubildungen der Geschlechtsorgane, die durch Adipositas begünstigt werden, im Vordergrund und sind für über 2.000 verlorene Erwerbsjahre (28\%) verantwortlich. Diabetes mellitus mit etwa $23 \%$ und Adipositas mit rund $20 \%$ folgen auf den weiteren Rängen.

Nachfolgend werden die indirekten Kosten aufgrund von Mortalität nach ICD 10 Ziffern für beide Geschlechter in gleicher Weise wie bereits bei der Berechnung der indirekten Kosten aufgrund von Invalidität dargestellt. Hierzu werden aufgrund der Komplexitätsreduzierung wiederum einheitliche, durchschnittliche Bruttoeinkommen (aus Teil- und Vollzeitarbeit) unselbständiger Arbeitnehmer des Bezugsjahres herangezogen und ein Gegenwartbezug durch die Diskontierung mit 4\% und $6 \%$ hergestellt. Die Tabellen der Sterbefälle und sämtliche darauf basierende Zwischenberechnungen finden sich - nach Geschlechtern getrennt - im Anhang dieser Arbeit.

Die indirekten Kosten für Adipositas in Höhe von 51.747.167,4 € (4\%) bzw. $37.156 .883,5 €(6 \%)$ werden mit dem beizumessenden Anteil von $100 \%$ angerechnet. Bei den übrigen Diagnosen werden die, bereits im Rahmen der Ermittlung der weiteren Kostenbestandteile, genutzten PAF-Relationen angesetzt. Als Ergebnis zeigt sich, dass die indirekten Kosten für Mortalität im Bereich von 313,3 
Mio. $€(4 \%)$ bzw. 234,5 Mio. $€(6 \%)$ liegen und somit etwa das Sechsfache der Kosten für Adipositas als Einzeldiagnose betragen. Die höchste Mortalität verbindet sich mit KHK und mit Diabetes mellitus, die zusammen für über $60 \%$ der Kosten verantwortlich sind. Dagegen weisen im Gegensatz zu den vorangegangenen Abschnitten Arthrose (1,8\% bzw. 2,3\%) und Depression (10,5\% bzw. 11,3\%) erwartungsgemäß die niedrigsten Werte auf, da diese Krankheitsbilder selten zum Tode führen.

Tabelle 21: Indirekte Kosten durch Mortalität (beide Geschlechter) ungewichtet und mit PAF gewichtet bei Diskontierungsraten $4 \%$ und $6 \%$.

\begin{tabular}{|c|c|c|c|c|c|}
\hline & $\begin{array}{c}4 \% \\
\text { Diskontierungs- } \\
\text { rate }\end{array}$ & $\begin{array}{c}6 \% \\
\text { Diskontierungs- } \\
\text { rate }\end{array}$ & PAF & $\begin{array}{c}4 \% \\
\text { Diskontierung } \\
\text { s-rate }\end{array}$ & $\begin{array}{c}6 \% \\
\text { Diskontierung } \\
\text { s-rate }\end{array}$ \\
\hline & ungewichtet & Ungewichtet & & Gewichtet & Gewichtet \\
\hline Erkrankung & $\begin{array}{c}\text { Beide } \\
\text { Geschlechter } \\
\text { In } €\end{array}$ & $\begin{array}{c}\text { Beide } \\
\text { Geschlechter } \\
\text { In } €\end{array}$ & $\ln \%$ & $\begin{array}{c}\text { Beide } \\
\text { Geschlechter } \\
\text { In } €\end{array}$ & $\begin{array}{c}\text { Beide } \\
\text { Geschlechter } \\
\text { In } €\end{array}$ \\
\hline Hypertonie & $134.066 .508,5$ & $102.292 .383,7$ & 9,7 & $1.300 .451,3$ & $9.922 .361,2$ \\
\hline Diabetes mellitus & $220.153 .155,8$ & $166.114 .234,7$ & 42,5 & $93.565 .091,2$ & $70.598 .549,7$ \\
\hline KHK & $1.842 .609 .111,2$ & $1.401 .550 .231,2$ & 5,5 & $101.343 .501,1$ & $77.085 .262,7$ \\
\hline Schlaganfall & $168.716 .326,6$ & $129.728 .708,2$ & 3,7 & $6.242 .504,1$ & $4.799 .962,2$ \\
\hline Colon CA & $279.566 .678,0$ & $215.719 .811,3$ & 3,0 & 8.387.000,3 & $6.471 .594,3$ \\
\hline CA $q$ Geschlechtsorgane & $274.831 .368,0$ & $202.344 .408,3$ & 10,3 & $28.307 .630,9$ & $20.841 .474,1$ \\
\hline Prostata CA & $57.009 .189,5$ & $48.084 .158,1$ & 1,6 & $912.147,0$ & $769.346,5$ \\
\hline Gallenblase/ Pankreas & $97.299 .817,1$ & $67.465 .893,1$ & 9,7 & $9.438 .082,3$ & $6.544 .191,6$ \\
\hline Arthrose & $62.313,0$ & $59.415,2$ & 9,1 & $5.670,5$ & $5.406,8$ \\
\hline Depression & $580.800,6$ & $464.966,8$ & 5,7 & $33.105,6$ & $26.503,1$ \\
\hline Schlafapnoe & $1.434 .817,1$ & $1.137 .859,2$ & 21,2 & $304.181,2$ & $241.226,2$ \\
\hline Adipositas & $51.747 .167,4$ & $37.156 .883,5$ & 100 & $51.747 .167,4$ & $37.156 .883,5$ \\
\hline Summe & 3.128.077.253,0 & 2.372.118.953,2 & -- & $313.290 .533,1$ & 234.462.761,9 \\
\hline
\end{tabular}

Quelle: $\quad$ Eigene Berechnungen auf Basis der angegebenen Quellen (gerundet)

\section{Zusammenfassung}

Zusammenfassend werden die drei Komponenten der indirekten Kosten mit unterschiedlichen Diskontierungsraten (nur angewendet für Invalidität und Mortalität) einander gegenübergestellt: 
Abbildung 6: Indirekte Kosten für Arbeitsunfähigkeit, Invalidität und Mortalität bei 4\% Diskontierungsrate in $1.000 €$ und \%

$760.743 € ; 46 \%$

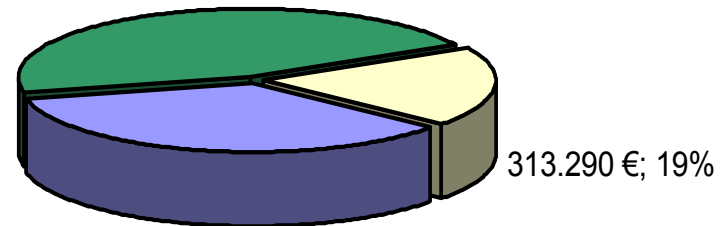

口Arbeitsunfähigkeit

口Invalidiät

口Mortalität

$582.242 € ; 35 \%$

Quelle:

Eigene Berechnungen auf Grundlage der angegebenen Daten.

Wird eine Situation im Gesundheitsmarkt vorausgesetzt, die einer 4\%-igen Diskontierung entspricht, so erhält man eine Gesamtbelastung an indirekten Kosten in Höhe von rd. 1,656 Mrd. €. Bei einer 6\%-igen Abzinsung ergibt sich zum Vergleich ein Gesamtwert von rd. 1,404 Mrd. $€$.

Abbildung 7: Indirekte Kosten für Arbeitsunfähigkeit, Invalidität und Mortalität bei 6\% Diskontierungsrate in $1.000 €$ und \%

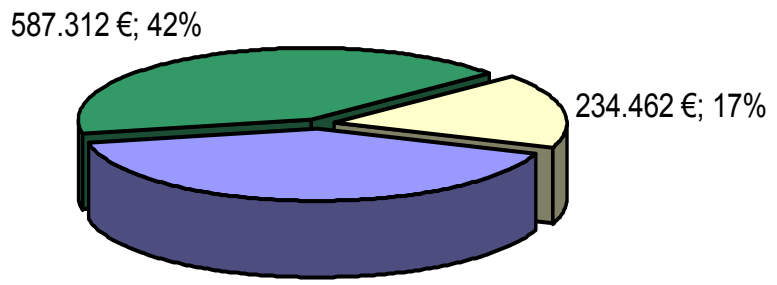

$\square$ Arbeitsunfähigkeit

口Invalidität

口Mortalität

$582.242 € ; 41 \%$

Quelle:

Eigene Berechnungen auf Grundlage der angegebenen Daten.

Die indirekten Kosten für Mortalität betragen bei einer Diskontierungsrate von 4\% rd. 313 Mio. $€$ und bei einer Diskontierungsrate von 6\% rd. 234 Mio. $€$ und weisen daher von allen indirekten Kostenanteilen den geringsten Wert auf. Ginge man davon aus, dass ein Teil der durch Tod ausfallenden Arbeitskräfte (im Sinne des Friktionskostenansatzes) durch andere Erwerbsfähige ersetzt würde, dann wäre 
der ökonomische Verlust durch arbeitsunfähige Personen kritischer zu bewerten, da aus dieser Perspektive ein Ersatz im Sinne einer Neueinstellung anderer Erwerbspersonen aufgrund nur befristeter Ausfälle nicht gegeben wäre. Der Humankapital-Ansatz berücksichtigt diesen Aspekt jedoch nicht. Im Vergleich zu den indirekten Kosten aufgrund von Invalidität in Höhe von 760,7 Mio. $€$ (bei 4\%) bzw. 587,3 Mio. $€$ (bei $6 \%$ ), die sich bei den unterschiedlichen Diskontierungsraten um etwa 173 Mio. $€(30 \%)$ unterscheiden, fallen sowohl Arbeitsunfähigkeit als auch Mortalität hinter das Ausmaß dieses Kostenaspekts zurück, so dass diese dauerhaften Arbeitsausfälle die größte Kostenbelastung mit sich bringen. Aufgrund der Diskontierung ist die Abweichung zwischen den Kosten für Invalidität und jenen nicht zu diskontierenden Kosten für Arbeitsunfähigkeit v.a. bei höheren Diskontierungssätzen (6\%) allerdings nahezu aufgehoben.

\subsubsection{Gesamtkosten}

Nachdem alle erforderlichen Einzelbestandteile der Kosten für Adipositas ermittelt werden konnten, kann im Rahmen dieses Abschnitts eine Konsolidierung auf Gesamtkostenebene vorgenommen werden. Es gilt zu berücksichtigen, dass durch die Verwendung von zwei unterschiedlichen Diskontierungssätzen (4\% und 6\%) im Falle der indirekten Kosten bei der Berechnung der Gesamtkosten ebenfalls zwei Werte zu unterscheiden sind. Zusätzlich zu den absoluten Kostenwerten der direkten und indirekten Kosten werden die relativen Anteile an den Gesamtkosten (100\%) aufgezeigt.

In der Darstellung erkennt man die klare Dominanz der direkten attributiven Kosten für die 11 Folge- und Begleiterkrankungen der Adipositas gegenüber den restlichen Kostenbestandteilen. Auf sie entfallen in beiden Diskontierungsversionen mit 86,6\% bzw. 88,3\% die größten Anteile der gesamten Kosten, die 13,066 Mrd. $€(4 \%)$ bzw. 12,754 Mrd. $€(6 \%)$ betragen. Es zeigt sich in eindrücklicher Weise, dass Adipositas selbst im Zuge der direkten Kosten mit gerade einmal $0,66 \%$ bzw. 0,67\% der Gesamtkosten eine geringe Rolle spielt, so dass auch die identifizierten Probleme aufgrund der unzureichenden Verfügbarkeit von Erhebungsdaten und Uneinheitlichkeiten in den Vergleichsstudien relativiert werden. Somit wird die eingangs aufgestellte These belegt, dass die assoziierten Erkran- 
kungen für die hohe Kostenbelastung verantwortlich sind, die sich sowohl in den direkten wie indirekten Kostenanteilen zeigen. Die Gesamtkosten entsprechen Anteilen von $0,61 \%$ bzw. 0,60\% am BIP in Höhe von 2.130 Mrd. € (gewähltes Bezugsjahr 2003).

Tabelle 22: Gesamtkosten (direkte/ indirekte) der Adipositas (in $1.000 €$ ) unter Berücksichtigung der Diskontierungsraten 4\% u. 6\% (Rundungsabweichungen).

\begin{tabular}{|c|c|c|c|c|c|c|}
\hline \multicolumn{2}{|c|}{$\begin{array}{l}\text { Direkte Kosten } \\
\quad \text { (in } 1.000 €)\end{array}$} & \multicolumn{4}{|c|}{ Indirekte Kosten (in $1.000 €$ ) } & \multirow[t]{2}{*}{$\begin{array}{l}\text { Summe } \\
\text { (in } 1.000 € \text { ) }\end{array}$} \\
\hline $\begin{array}{c}\text { Adipositas } \\
\text { ICD } 10 \mathrm{E} \\
65-68\end{array}$ & $\begin{array}{c}\text { Assoziierte } \\
\text { Erkrankungen }\end{array}$ & $\begin{array}{c}\text { Arbeits- } \\
\text { unfähigkeit }\end{array}$ & $\begin{array}{l}\text { Diskon- } \\
\text { tierungs- } \\
\text { satz }\end{array}$ & Invalidität & Mortalität & \\
\hline $85.710 €$ & $11.265 .000,0 €$ & $582.242,5 €$ & $4 \%$ & $\begin{array}{r}760.743,0 \\
€\end{array}$ & $313.290,0 €$ & $13.006 .985 €$ \\
\hline $0,66 \%$ & $86,61 \%$ & $4,48 \%$ & & $5,85 \%$ & $2,41 \%$ & $100 \%$ \\
\hline \multicolumn{2}{|c|}{$11.350 .710 €$} & \multicolumn{4}{|c|}{$1.656 .275,5 €$} & $13.066 .985 €$ \\
\hline \multicolumn{2}{|c|}{$86,87 \%$} & \multicolumn{4}{|c|}{$12,68 \%$} & $100,00 \%$ \\
\hline \multicolumn{2}{|c|}{$\begin{array}{l}\text { Direkte Kosten } \\
\text { (in } 1.000 €)\end{array}$} & \multicolumn{4}{|c|}{ Indirekte Kosten (in $1.000 €$ ) } & $\begin{array}{c}\text { Summe } \\
\text { (in } 1.000 € \text { ) }\end{array}$ \\
\hline $\begin{array}{c}\text { Adipositas } \\
\text { ICD } 10 E \\
65-68\end{array}$ & $\begin{array}{c}\text { Assoziierte } \\
\text { Erkrankungen }\end{array}$ & $\begin{array}{l}\text { Arbeits- } \\
\text { unfähigkeit }\end{array}$ & $\begin{array}{l}\text { Diskon- } \\
\text { tierungs- } \\
\text { satz }\end{array}$ & Invalidität & Mortalität & \\
\hline $85.710 €$ & $11.265 .000,0 €$ & $582.242,5 €$ & $6 \%$ & $\begin{array}{r}587.312,0 \\
€\end{array}$ & $234.462,0 €$ & $12.754 .726 €$ \\
\hline $0,67 \%$ & $88,32 \%$ & $4,56 \%$ & & $4,60 \%$ & $1,84 \%$ & $100 \%$ \\
\hline \multicolumn{2}{|c|}{$11.350 .710 €$} & \multicolumn{4}{|c|}{$1.404 .016,5 €$} & $12.754 .726 €$ \\
\hline \multicolumn{2}{|c|}{$88,98 \%$} & \multicolumn{4}{|c|}{$11,01 \%$} & $100 \%$ \\
\hline
\end{tabular}

Quelle: $\quad$ Eigene Berechnungen auf Grundlage der angegebenen Daten.

Bei getrennter Darstellung der unterschiedlichen Diskontierungsszenarien ergeben sich nachfolgende Abbildungen. Sie veranschaulichen beide den zentralen Stellenwert der attributiven direkten Kosten von Adipositas. 
Abbildung 8: $\quad$ Direkte und indirekte Kosten im Vergleich bei einer Diskontierungsrate von $4 \%$ (in \%)

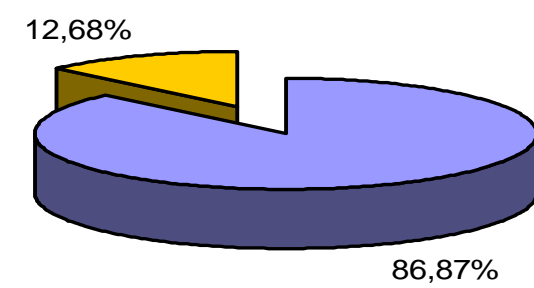

Bei einer Diskontierung von $4 \%$ betragen die gesamten direkten Kosten mit einem Anteil von über 11,350 Mrd. € und 86,87\% knapp das Siebenfache der gesamten indirekten Kosten, die 1,656 Mrd. € und 12,68\% repräsentieren. Nur geringfügig verändert zeigt sich das Gesamtbild im Falle einer Diskontierung mit 6\%. Auch hier fallen die direkten attributiven Kosten in besonderer Weise ins Gewicht und verändern die Relation zwischen den gesamten direkten Kosten mit einem Anteil von $88,89 \%$ oder 11,350 Mrd. $€$ und den indirekten Kosten, mit $11,01 \%$ oder 1,404 Mrd. $€$, um nur wenige Prozentpunkte.

Abbildung 9: $\quad$ Direkte und indirekte Kosten im Vergleich bei einer Diskontierungsrate von $6 \%$ (in \%)

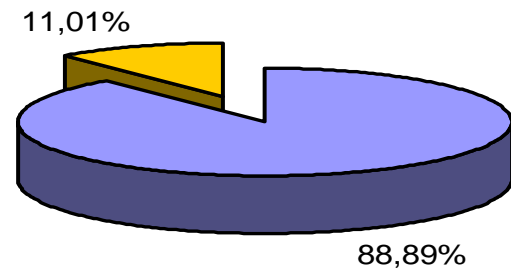

Quelle: $\quad$ Eigene Berechnungen auf Grundlage der angegebenen Daten.

Eine noch detaillierter dargestellte Gesamtkostensicht beider Diskontierungsszenarien findet sich ergänzend im Anhang der Arbeit. 
Vergleicht man die Ergebnisse z.B. mit jenen für die Schweiz aus dem Jahr 2001, die - nur auf Adipositas bezogen - unter zumindest ähnlichen Berechnungsbedingungen mit 1,316 Mrd. SFr. (etwa 850 Mio. $€$ ) angegeben werden, liegen die Kosten in Deutschland um rund das 15-fache höher als die Kosten in der Schweiz. ${ }^{122}$ Legt man eine Bevölkerungszahl von etwa 7,4 Mio. für die Schweiz (allerdings ohne nähere Betrachtung der Prävalenzen von Adipositas) und 82,4 Mio. für Deutschland zugrunde, beträgt diese Relation rund das 11-fache. Aus dieser Überlegung heraus ermittelt sich eine Pro-Kopf-Belastung bezogen auf die Gesamtbevölkerung in Deutschland von rund $156 €$ (Schweiz $114 €$ ) und bezogen auf rund 13,2 Mio. adipöse Personen von etwa $975 €$ (Schweiz: $1.785 €$ ). Die von der OECD ermittelten Pro-Kopf-Ausgaben allerdings zeigen, dass die Menschen in Deutschland mit 3.287 US \$ um 890 US \$ (etwa $580 €$ ) weniger für Gesundheit ausgeben als die Schweiz mit 4.177 US \$. Dieser Aspekt trägt dazu bei, die Differenz im Zusammenhang mit den Pro-Kopf-Ausgaben zwischen Deutschland und der Schweiz für Adipositas zu relativieren. ${ }^{123}$ Nichtsdestotrotz bleiben die hier kalkulierten Pro-Kopf-Ausgaben für Deutschland um etwa $230 €$ geringer als jene für die Schweiz. Für weitere vergleichende Aussagen und Zusammenhänge, wären - v.a mit Blick auf die Prävalenzen und Kostenstrukturen des Landes tiefer gehende Untersuchungen erforderlich, die im Rahmen dieser Arbeit jedoch nicht durchführbar sind. Weitere ausführlichere Vergleiche zu den USA und Großbritannien werden gesondert in einem eigenen Abschnitt (3.4) betrachtet.

Ein weiterer Vergleich kann zu den ermittelten gesundheitsökonomischen Kosten des Rauchens aus dem Jahr 1996 (jüngere Untersuchungen in dieser Ausführlichkeit waren leider nicht aufzufinden) in Höhe von 35,2 Mrd. € (4\% Diskontierungsrate) und $33,5 \mathrm{Mrd} . €$ (6\% Diskontierungsrate) vorgenommen werden. ${ }^{124}$ Rauchen weist im Rahmen dieser Untersuchung eine noch höhere Kostenbelastung auf als Adipositas. Ohne auch hier genauer auf die detaillierten Zusammenhänge eingehen zu können, lässt sich mit Blick auf die gegenwärtig geführten

\footnotetext{
${ }^{122}$ Schneider, H./ Schmidt, A. (2004).

${ }^{123}$ OECD Gesundheitsdaten 2007, http://www.oecd.org/dataoecd/15/1/39001235.pdf.
} 
Diskussionen um Rauch- und Werbeverbote sowie Maßnahmen zur Raucherprävention dennoch die Schlussfolgerung ziehen, dass Gesundheits- und somit Kostenbelastungen spätestens in dieser Höhe sowohl in der Politik als auch in der Gesellschaft zu intensivierten Debatten und Handlungsschritten führen. Allerdings sollte bei diesem Vergleich nicht übersehen werden, dass Rauchen mit dem ökonomischen Problem negativer externer Effekte für andere Personen (Passivrauchen) einhergeht, was im Falle von Adipositas so gut wie keine Rolle spielt. Nichtsdestotrotz bedingt die Kostenbelastung von Adipositas Konsequenzen, die v.a. in einer verstärkten Bemühung um die Vermeidung/ Prävention von daraus entstehenden Gesundheitsdefiziten und Kosten zu erkennen sein sollten.

\subsection{Kostenaspekte der Adipositasprävention}

\subsubsection{Initiativen zur Gesundheitsförderung und Prävention}

In Anbetracht der ermittelten Kostenbelastung durch Adipositas und der Perspektive, ohne weitere Maßnahmen, zukünftig mit großer Wahrscheinlichkeit steigende Prävalenzen zu verzeichnen, ${ }^{125}$ richtet sich der Blick auf Möglichkeiten einer Vermeidung von Übergewicht und Adipositas.

Faktisch sind, nach Aussage des Sachverständigenrats (SVR) zur Begutachtung der Entwicklung im Gesundheitswesen, alle Präventionsprogramme an festgelegten Voraussetzungen zu messen, die gerade aus Kostensicht von zentraler Bedeutung sind und im Bezug auf Gesundheitsförderung und primärer Prävention wie folgt vorgegeben werden: ${ }^{126}$

- die Häufigkeit der zu vermeidenden Erkrankung soll gegeben sein

- die medizinische Relevanz soll belegt sein

- die Wirksamkeit der Vorbeugungsmaßnahmen soll sichergestellt sein

- die Maßnahmen sollen mit vertretbaren Kosten einhergehen

\footnotetext{
124 Ruff, L. (2000).

${ }^{125} \mathrm{Vgl}$. WHO (2005) in Verbindung mit den Ausführungen in Abschnitt 3.3.

${ }^{126}$ Vgl. im Folgenden Sachverständigenrat (2000): Gesundheitsversorgung und Krankenversicherung 2000 , S. $21 \mathrm{ff}$.
} 
- Präventionsmaßnahmen selbst sollen ein vernachlässigbares Risiko aufweisen.

Des Weiteren wird vom SVR gefordert, dass Gesundheitsförderung und generelle, alle Bevölkerungsteile betreffende primäre Präventionsmaßnahmen soweit erforderlich aus allgemeinen Mitteln finanziert werden sollten. Dagegen sei individuelle primäre, sekundäre und tertiäre Prävention, die auf die Früherkennung sowie auf die Vermeidung der Verschlechterung von Krankheiten abzielt, ärztliche Aufgabe und überwiegend von den Krankenkassen zu finanzieren. Dies gelte insbesondere für die individuelle Gesundheitsberatung, die im Rahmen der GKV altersunabhängig vorzugsweise von Hausärzten durchgeführt und als zeitintensive, zielgerichtete und persönliche Beratung angemessen vergütet werden sollte. ${ }^{127}$

Erfolge von Präventionsmaßnahmen werden über Langzeitstudien herausgefunden und entscheiden wesentlich über dahingehend weitere Investitionen. Aus diesem Grund erscheint es sinnvoll, einige bereits existierende Programme kritisch zu betrachten. Mit Blick auf die Epidemiologie von Kindern und Jugendlichen mit Übergewicht und Adipositas und der damit einhergehenden verlängerten Risikoexposition steht v.a. diese Zielgruppe für Präventionsansätze im Vordergrund. ${ }^{128}$ In einer diesbezüglich durchgeführten, umfassenden Metaanalyse aus dem Jahr 2005 konnten national und international 19 Studien und 5 Reviews ausgewertet werden. ${ }^{129}$ Die Kosten der Programme wurden im Rahmen der Untersuchungen leider nicht berücksichtigt. Die dortigen Ergebnisse zeigen, dass nicht prinzipiell von einem Erfolg der Prävention ausgegangen werden kann, jedoch v.a. länger andauernde schul- und familienbasierte Interventionsprogramme Effektivität versprechen, die Einfluss auf adipositas-fördernde Verhältnisse und adipositas-förderndes Verhalten nehmen. Die Forderung der Analyse geht allerdings dahin, dass weitere fundierte Studien mit längeren Laufzeiten aufgesetzt werden sollten, um neben der Wirksamkeit auch die Nachhaltigkeit derar-

\footnotetext{
${ }^{127}$ Vgl. ebenda, S. 22.

${ }^{128}$ Vgl. Hauner, H et al. (2007), S. 9.

${ }^{129}$ Vgl. Schaff, M./ Handel, U. (2005): Literaturübersicht zu Adipositasprävention im Kindesalter.
} 
tiger Maßnahmen belegen zu können. Grundsätzlich ist dieser Forderung eine weitere hinzuzufügen, nämlich dass Kosten-Nutzen-Betrachtungen aller Einzelmaßnahmen begleitender Bestandteil der Untersuchungen sein sollte, wie dies z.B. im Rahmen der Kieler Adipositas-Präventionsstudie KOPS, eine seit 1996 durchgeführte Langzeituntersuchung, die noch bis in das Jahr 2009 weitergeführt werden wird, umgesetzt wird. ${ }^{130}$

Eine weitere nennenswerte Initiative wird, unter der Bezeichnung "Nationaler Aktionsplan gegen das Übergewicht", seitens der Deutschen Adipositas-Gesellschaft betrieben, die sich damit an den internationalen Vorgaben der EU („Green paper") und der WHO (Ministerkonferenz im Kampf gegen Adipositas) orientiert. ${ }^{131}$ Die Gesellschaft beruft sich dabei auf Implementierungen und erste medizinischpräventive Erfolge in einigen Ländern Europas wie Dänemark, Irland, Portugal, Slovakei und Spanien. Zwar werden darin ausführlich die Ziele und Strategien des Plans beschrieben, jedoch finden sich keine Kosten- oder Finanzierungsangaben, die eine finanzielle Kalkulation für eine Umsetzung in Deutschland zuließen.

Mit dem Präventionsbericht 2007 wird die Transparenz von Leistungen der gesetzlichen Krankenversicherungen in der Primärprävention und der betrieblichen Gesundheitsförderung dokumentiert. Es zeigt sich, dass (für das Berichtsjahr 2006) Primärprävention und Gesundheitsförderung wesentliche Teilbereiche einer Förderung der Präventionsförderung durch die GKV darstellen. In diesem Rahmen finden u.a. Vorsorgeuntersuchungen, Schutzimpfungen oder die zahnmedizinische Prophylaxe statt, ein Engagement, welches durch die $\S \S 20$ und 20a SGB V als eines der Aufgabengebiete der GKV festgelegt ist. Dabei werden die Bereiche Primärprävention und Gesundheitsförderung über drei Ansätze abgedeckt: ${ }^{132}$

\footnotetext{
${ }^{130}$ Vgl. Czerwinski-Mast et al. (2003): Kieler Adipositaspräventionsstudie (KOPS).

${ }^{131} \mathrm{Vgl}$. Müller, M. J. et al. (2007),

${ }^{132}$ Vgl. Medizinischer Dienst der Spitzenverbände der Krankenkassen e.V. (MDS): Präventionsbericht 2007.
} 
- Settingansatz mit Ansatzpunkten in den frequentierten Lebensumfeldern der Versicherten, wie z.B. in Schulen, Kindergärten, Seniorenheimen oder auch Familien

- Individueller Ansatz als Angebot von primärpräventiven Kursen und Seminaren für Einzelversicherte

- Betriebliche Gesundheitsförderung (BGF), zur Förderung von identifizierten Gesundheitspotenzialen in Unternehmen und zur Unterstützung eines gesundheitsbewussten Verhaltens der Belegschaften

Die eingesetzten finanziellen Mittel für die Primärprävention und betriebliche Gesundheitsförderung werden in einer Höhe von 232 Millionen Euro angegeben. Bezogen auf die Anzahl der Versicherten entspricht diese Summe einem Anteil von $3,30 €$ je Versicherten, welcher absolut gesehen gering erscheinen mag, allerdings dennoch das gesetzlich angestrebte Ausgabensoll von 2,74€ je Versicherten übertrifft. Dabei stehen Präventionsaspekte wie „Bewegung“, „Ernährung“, „Stressreduktion“ sowie der „Umgang mit Genuss- und Suchtmitteln“ im Vordergrund. Die Durchführung einer Erfolgskontrolle der Maßnahmen wird in einem Umfang von 43\% angegeben. In etwa einem Viertel der Maßnahmen (26\%) ist keinerlei Erfolgskontrolle vorgesehen und in den übrigen Fällen (31\%) ist diese zumindest geplant. Die weiteren Aktivitäten für die nächsten Jahre haben das Ziel, unter Einbeziehung des unabhängigen Sachverstandes und Experten aus den Bereichen Epidemiologie, Präventionsforschung, im Rahmen der nichtbetrieblichen Gesundheitsförderung die „Reduktion von Krankheiten des Kreislaufsystems" zu bewirken. Der Schwerpunkt dieser Maßnahmen zielt in erster Linie auf Kinder, Jugendliche und junge bzw. werdende Eltern ab.

Andere Überlegungen gehen dahin, dass weitere Präventionsoptionen z.B. durch ordnungspolitische Maßnahmen gegeben sind. ${ }^{133} \mathrm{Da}$ - wie bereits bei der Berechnung von indirekten Kosten durch die Diskontierung unterstellt - im Allgemeinen davon ausgegangen wird, dass Werte von Individuen (z.B. Gesundheitszustand, Konsumgüter) in der Gegenwart höher eingeschätzt werden als in der Zukunft

${ }^{133}$ Vgl. Hebebrand, J. (2004). S. $2473 f$. 
liegende mögliche Konsequenzen (z.B. die Entstehung von Adipositas und Folgeerkrankungen), sind unterschiedliche ordnungspolitische Ansätze denkbar: ${ }^{134}$

Eine Option wäre z.B. die Verteuerung der relevanten Gegenwartswerte und dadurch die Korrektur der zeitlich abweichenden Präferenzen auf Nachfrageseite über eine hohe indirekte Besteuerung „ungesunder" Nahrungsmittel (Problem der Selektion). Des Weiteren ließen sich auf der Anbieterseite ggf. ökonomische Anreize (Steuerersparnisse/ Subventionen) für die Nahrungsmittelindustrie schaffen, um die Herstellung und Bewerbung von Nahrungsmitteln zu fördern, die geringeren Einfluss auf die Entstehung von Adipositas haben (Problem der Selektion). Die Umsetzbarkeit derartiger Maßnahmen erscheint zum einen aufgrund von Marktdynamik und zu erwartenden Widerständen der Zielgruppen wenig realistisch zu sein. Zum anderen ist es fraglich, ob die Ordnungspolitik in diesem Zusammenhang überhaupt sinnvoll ist und es nicht angebrachter wäre, sich verstärkt auf Maßnahmen zu konzentrieren, welche die Selbstverantwortung und kognitive Einsicht für das Problem Adipositas in der Bevölkerung fördern, wie z.B. die Motivation zur körperlichen Aktivität (Förderung von Schulsport/ Sportvereinen), wie es derzeit beispielsweise mit der bundesweiten Kampagne „Deutschland bewegt sich" der Fall ist. ${ }^{135}$

Weitere von der breiteren Öffentlichkeit wahrgenommenen Maßnahmen in dieser Richtung werden bislang in erster Linie von den GKV und gemeinsamen Initiativen aus Politik, Verbänden und Wirtschaft, wie der „Plattform Ernährung und Bewegung e.V.", als bedeutendste Vereinsinitiative innerhalb der EU, getragen. Sonstige Aktivitäten über kleinere Organisationen, Initiativen oder Verbände sind nur schwer zu identifizieren. Aus diesen Gründen können die gesamten Kosten für Gesundheitsförderung und Prävention im Allgemeinen und diejenigen für Adipositas im Speziellen, grundsätzlich nur grob abgeschätzt werden. Derzeit wird davon ausgegangen, dass alle Maßnahmen dieser Art in Deutschland einen vergleichsweise geringen Anteil von ca. 4,5\% (etwa 10,5 Mrd. €) der gesamten

${ }^{134}$ Vgl. ebenda. 
öffentlichen und privaten Gesundheitsausgaben, die in 2004 rd. 234 Mrd. $€$ betrugen, repräsentieren. ${ }^{136}$

Die Bundesregierung hat in einem Gesetzesentwurf aus 2007 erforderliche Mehrausgaben für ein Gesamtprogramm zur „Stärkung der Gesundheitsförderung und gesundheitlicher Prävention“ kalkuliert und kommt dabei auf folgende Aufstellung:

Tabelle 23: Kalkulierte Mehrausgaben für Gesundheitsförderung und Prävention der Bundesregierung

\begin{tabular}{|c|c|}
\hline Kostenträger Prävention & $\begin{array}{c}\text { Mehrausgaben } \\
\text { In Tausend } €\end{array}$ \\
\hline Bund (Berichterstattung) & 206 \\
\hline Gesetzliche Krankenversicherung & 250.000 \\
\hline Gesetzliche Rentenversicherung & Keine \\
\hline Soziale Pflegeversicherung & 14.300 \\
\hline Gesetzliche Unfallversicherung & 28.600 \\
\hline Summe & 293.106 \\
\hline
\end{tabular}

Quelle: Zusammenstellung/ Teilberechnung aus dem Entwurf eines Gesetzes zur Stärkung der Gesundheitsförderung und gesundheitlicher Prävention sowie zur Änderung anderer Gesetze. Bundesregierung, 23.11.2007, veröffentlicht unter: http://www.gesundheitberlin.de/download/Referentenentwurf Praeventionsgeset z 11 2007.pdf.

Ungeachtet der Situation, dass das geplante Präventionsgesetz politisch bislang nicht umgesetzt werden konnte, werden erforderliche Mehrausgaben für Präventionsmaßnahmen in einer jährlichen Gesamthöhe von rund 293 Mio. € angegeben. Ein konkreter Anteil, welcher davon spezieller Adipositas-Prävention zuzurechnen wäre, wird als solches nicht beziffert. In den für Deutschland erarbeiteten Gesundheitszielen finden sich jedoch zumindest Anforderungen, die indirekt auf

\footnotetext{
${ }^{135}$ Die Initiative wurde 2003 gestartet und wird von der Krankenkasse Barmer, dem ZDF und Bild am Sonntag gefördert.

${ }^{136}$ Vgl. Apitz, R./ Winter, S.F. (2003): www.bmg.bund.de/.../artikelpraevention-pdf-5711.pdf. S. 4. , in Verbindung mit den Angaben des Statistischen Bundesamtes aus 2006, unter www.destatis.de.
} 
die Vermeidung von Adipositas als krankheitsbegünstigende Ursache abzielen, wie u.a. ${ }^{137}$

- die Senkung des Erkrankungsrisikos von Diabetes mellitus Typ 2,

- Gesund aufwachsen: Ernährung, Bewegung, Stressbewältigung

- Erhöhung gesundheitlicher Kompetenz und Stärkung der Patientensouveränität

Aus diesem Grund ist ein wesentlicher Teil der Ausgaben (neben jenen für Raucherprävention und Prävention von häufigen Krebserkrankungen) der Prävention von Adipositas und den wichtigsten assoziierten Erkrankungen, v.a. Diabetes mellitus, zuzuschreiben. Inwieweit die geplanten Ausgaben in ihrer Höhe ausreichen können, Adipositas und Folge- und Begleiterkrankungen wesentlichen Einhalt zu gebieten, kann nur durch langfristig angelegte Studien belegt werden und lässt sich gegenwärtig nicht konkret benennen. Setzt man Vergleichszahlen der Raucherprävention an, so wird dort davon ausgegangen, dass nach einer Latenzzeit von rund 10 Jahren etwa 7,7\% an direkten Kosten vermieden werden könnten. ${ }^{138}$ Im Falle der Adipositas könnte daraufhin eine eher konservativ angenommene Reduzierung der ermittelten gesamten direkten Kosten, von beispielsweise $5 \%$, rund 900 Mio. $€$ an Einsparungspotenzial bedeuten. Dabei sollte zum einen allerdings nicht übersehen werden, dass sämtliche Kalkulationen dieser Art aufgrund der unzureichenden v.a. wirtschaftlichen Rahmendaten hohen Unsicherheiten unterliegen. Zum anderen besteht auch hier die Problematik, dass vermeintliche Einsparungspotentiale als alleinige Bewertung des ökonomischen Erfolgs der Maßnahmen nicht als ausreichend anzusehen sind und originär nichtmonetäre Aspekte (z.B. Lebensqualität, gesellschaftliche Akzeptanz usw.) erneut zu thematisieren wären.

\subsubsection{Nutzen-Kosten-Relationen der Prävention}

Ökonomische Nutzen-Kosten- bzw. Kosten-Effektivitäts-Analysen zur Bewertung von präventiven Adipositasinitiativen sind, wie sich gezeigt hat, mit bekannten

\footnotetext{
${ }^{137}$ Vgl. Gesundheitsziele.de, veröffentlicht unter: http://www.bmg.bund.de.

${ }^{138}$ Vgl. Plamper, E. (2005).
} 
und nur bedingt lösbaren Problemen, v.a. einer sinnvollen monetären Bewertung der sich einstellenden Effekte, wie z.B. Krankheitsvermeidung oder Lebenszeitverlängerung, verbunden. Die Einschätzung z.B. von Kompetenzentwicklung oder auch einer steigenden gesundheitlichen Zufriedenheit von Risikogruppen lassen sich punktuell zwar erfassen und können in Bemessungen einfließen, jedoch existieren erhebliche Bewertungsprobleme, die bislang ungelöst sind. ${ }^{139}$ Auf vergleichbare Aspekte und Zusammenhänge wurde bereits im Zusammenhang mit den sog. „intangiblen Kosten“ hingewiesen, die im Rahmen dieser Arbeit zwar erwähnt, jedoch nicht weiter berücksichtigt werden können. Erschwerend äußert sich die Tatsache, dass sich die durch die verschiedenen Einzelmaßnahmen definierten Kosten für Prävention und Gesundheitsförderung zur Vermeidung von Adipositas zum Teil mit bestehenden Therapiemaßnahmen überschneiden und nicht exakt von diesen abzugrenzen sind. Je nach Risiko, Stadium und Ausprägung von Übergewicht oder Adipositas kann die medikamentöse Behandlung (z.B. anfangs durch Statine, in fortgeschritteneren Stadien zusätzlich durch Orlistat) zugleich eine präventive Maßnahme wie auch eine Therapieoption sein. Ebenso verhält es sich bei Ernährungsberatung, Beratungen zu Verhaltensänderungen sowie der Anleitung zu Bewegungs- und Sportprogrammen.

Hinzu kommt, dass die Adipositasprävention i.d.R. durch mehrere Akteure getragen wird und dadurch in der mittel- bis langfristigen Betrachtung der Effekte erhebliche Schwierigkeiten bei der Kostenabgrenzung und der Erfassung indirekter Wirkungseffekte entstehen, die aber dennoch berücksichtigt werden sollten. ${ }^{140}$ Letztlich wird die positive (oder negative) Beurteilung - trotz aller berechtigten Kritik - am ehesten über einen Rückgang (bzw. über die Stagnation/ Zunahme) von direkten und indirekten Kosten gegeben sein. Einzelmaßnahmen lassen sich dadurch zwar kaum beurteilen, doch zeigt sich dadurch in jedem Fall ein richtungweisender Trend der Präventionsbemühungen insgesamt.

Die Nutzen-Kosten-Relationen einzelner Initiativen zur Prävention und Gesundheitsförderung zur Vermeidung von Adipositas (und damit verbundener Gesund-

${ }^{139}$ Vgl. Hebebrand, J. (2004), S. 2471. 
heitsrisiken) lassen sich nur über den Weg studienbezogener Einzelbetrachtungen festmachen. Der Nettonutzen bzw. die Nettokosten der Präventionsmaßnahmen errechnen sich dabei aus den jeweiligen Gesamtkosten abzüglich der Kosten der vermiedenen (assoziierten) ICD 10-Erkrankungen, auf welche die Maßnahmen abzielen. Die Kosten vieler Präventionsmaßnahmen sind allerdings nicht in genügender Weise transparent, als dass sie zum Zwecke einer Gesamtkalkulation zugänglich und verfügbar wären. Somit ist zu fordern, dass die durchgeführten oder geplanten Maßnahmen nicht nur wie von SVR formuliert mit „vertretbaren Kosten einhergehen“, sondern auf eine Weise kalkuliert, geplant und offen gelegt werden, dass mit Blick auf die Bewertung von Kosten und Nutzen - neben einer Beurteilung ihres medizinischen Erfolgs oder Misserfolgs - ein gezieltes Controlling und eine Einschätzung der wirtschaftlichen Effizienz über ihre Gesamtdauer möglich ist. Diese Ausführungen machen einmal mehr deutlich, dass die Anforderung an eine strukturierte Datenerfassung einer der Hauptaspekte in diesem Zusammenhang darstellt.

\subsection{Entwicklungsszenarien und Hochrechnungen}

Die Bevölkerung in der Bundesrepublik Deutschland weist seit dem Jahr 1997 Wachstumsraten auf, die zwischen 0,0 und 0,2 Prozent liegen, bei einem etwa gleich bleibenden Ausländeranteil von 8,9\%. Während der Altenquotient über die Jahre kontinuierlich angestiegen ist (2003: 44,9\%) ${ }^{141}$, stagniert oder sinkt der Jugendquotient (2003: 37,3\%) ${ }^{142}$ und wies einen geringen Anstieg zuletzt in den Jahren 1997-1999 auf. ${ }^{143}$ Darüber hinaus werden in der offiziellen „Bevölkerungsvorausberechnung" des Statistischen Bundesamtes Annahmen zur Geburtenhäufigkeit, Lebenserwartung sowie zum Wanderungssaldo der Bevölkerung einbezogen und zu denkbaren Entwicklungsszenarien kombiniert. ${ }^{144}$ Denkt man allerdings an die im Rahmen des Humankapital-Ansatzes getroffenen Annahmen zur Ermittlung der indirekten Kosten, die lediglich erwerbsfähige Bevölkerungsanteile im Altersintervall zwischen 15 und 65 Jahren berücksichtigen, spielt die generelle

\footnotetext{
${ }^{140} \mathrm{Vgl}$. ebenda.

${ }^{141} 60$ jährige und ältere je 10020 - bis unter 60jährige.

142 Unter 20jährige je 100 20- bis unter 60jährige.

${ }^{143}$ Statistisches Bundesamt (2003): Bevölkerungsfortschreibung.
} 
Lebenserwartung zur Beurteilung der Kosten von Adipositas eine untergeordnete Rolle. Die verbleibenden Aspekte Geburtenhäufigkeit und Wanderungssaldo werden aufgrund geringer Veränderungsraten ebenfalls nicht bestimmend für zukünftige Adipositasentwicklungen bzw. Gesundheitskosten angesehen. Im Falle der Geburtenhäufigkeit wäre ggf. die statistische Maximalvariante eines Geburtenanstiegs von gegenwärtig 1,4 auf 2,1 Geburten je Frau mit Konsequenzen verbunden, wenngleich die Entwicklungen der vergangenen Jahre eher für eine Konstanz oder einen Rückgang an Geburten sprechen. Die als wahrscheinlich angenommenen Wanderungssalden bewegen sich in den Szenarien zwischen 0 und 300.000 Personen im Jahr, die nach Deutschland immigrieren. Inwieweit sie zu einer Zunahme der Adipositasentwicklung beitragen könnten, ist ohne genauere Analyse dieser Bevölkerungsgruppen nicht zu bestimmen und bleibt hier demnach ohne Konsequenz.

Da das größte Problem in der absoluten Unsicherheit zukünftiger Entwicklungen zu sehen ist, werden Fragen aufgeworfen, die sich - wenn überhaupt - nur tendenziell durch die Zuhilfenahme von vereinfachenden Annahmen beantworten lassen:

- Welche quantitativen Ausmaße nimmt Adipositas zukünttig an?

- Wie wird die zukünftige Adipositasverteilung in der Bevölkerung sein?

- Wie entwickelt sich die Bevölkerung v.a. in Bezug auf ihr Konsumverhalten, Gesundheits- und Ernährungsbewusstsein?

- Welchen aktiven sportlichen Betätigungen wird nachgegangen?

- Rücken weitere/ neuere medizinische Erkenntnisse über Erkrankungen und somit Kosten ins Blickfeld, die ergänzende Entstehungs-/ Progressionsrisiken durch die Existenz von Adipositas aufzeigen?

- Welche Regulierungsmechanismen werden ergriffen und ggf. welche Wirkungen zeigen sie?

Die Liste offener Fragen könnte noch um zahlreiche weitere Aspekte erweitert werden, die zum gegenwärtigen Zeitpunkt noch nicht konkret abzusehen sind.

${ }^{144}$ Statistisches Bundesamt (2006) 
Nichtsdestotrotz bewegt man sich bei dem Versuch, Entwicklungsszenarien zu erstellen, nicht völlig im Dunkeln, da zum einen retrospektive Sichtweisen und damit verbundene Tendenzen der vergangenen Jahre Anhaltspunkte geben können, wie sich die Situation von Adipositas in Deutschland - unter bestimmten Annahmen - in den nächsten Jahren darstellen könnte. Zum anderen existieren Hochrechnungen, die in erster Linie seitens der WHO angestellt werden bzw. weitere, die sich auf das gesamte Gesundheitssystem beziehen und Ableitungen für den Themenkomplex der Adipositas zulassen. ${ }^{145}$

Zur Verringerung der Hochrechnungsunsicherheiten können Ergebnisse aus Initiativen, wie z.B. der umfassenden „Nationalen Verzehrsstudie II“ der Bundesregierung herangezogen werden. ${ }^{146}$ Aus ihr lassen sich zwar keine direkten Kostenentwicklungen ableiten aber dafür liefert die Studie repräsentative Daten, welche Aufschluss über die Ernährungssituation der Bevölkerung Deutschlands geben. Aus langfristig angelegten Auswertungen dieser Art lassen sich konkretere Tendenzen ableiten, die prognostisch genutzt werden können. Die seit 2003 laufende Initiative analysiert z.B. Ernährungsweise, Einschätzungen des persönlichen Energiebedarfs, Koch- und Einkaufsverhalten, Ernährungswissen oder auch die Risikowahrnehmung der Bevölkerung für ernährungsbedingtes Fehlverhalten und damit einher gehende Entstehung oder Förderung von Adipositas. Eine genaue Auswertung und Berücksichtigung der Einzelergebnisse aus dieser oder vergleichbaren Studien ist im Rahmen dieser Ausarbeitung nicht zu leisten, da mit steigender Anzahl von Einflussgrößen die Komplexität von erforderlichen Rechenmodellen drastisch zunimmt. Zu diesem Zweck wäre eine eigenständige Analyse durchzuführen. In dem sich hier bietenden Rahmen werden bereits aggregierte Größen - wie das Bruttoinlandsprodukt - herangezogen, an denen sich selektive Szenarien festmachen lassen.

Auf der Basis aller Erkenntnisse dieser Arbeit ist die größte Bedeutung für zukünftige Kostenbelastungen weniger in der Entwicklung der absoluten Bevölkerungs-

\footnotetext{
${ }^{145}$ Z.B. Schlander, M. et al. (2005).

146 Nationale Verzehrsstudie II des Bundesministeriums für Ernährung, Landwirtschaft und Verbraucherschutz (BMELV), Karlsruhe 2003.
} 
zahlen zu sehen, als vielmehr in der Prävalenz von Adipositas. Dies gilt v.a mit Blick auf die Gesamtkosten und die Zusammenhänge massiv steigender ProKopf-Ausgaben adipöser und insbesondere stark adipöser Personen. Selbst bei konstanten oder rückläufigen Bevölkerungszahlen ist bei Erhöhung der Adipositasprävalenz von signifikanten Kostensteigerungen auszugehen. Ob und inwieweit allerdings die Prävalenzen über die kommenden 10 bis 15 Jahre tatsächlich in gleich bleibendem Maße ansteigen ist ungewiss. Eine Fortschreibung der bisher ermittelten Entwicklungen und Kosten wird daher bis zum Jahr 2020 durchgeführt. Darüber hinaus gehende Berechnungen erscheinen aus gegenwärtiger Sicht nicht sinnvoll, da zum einen die unterstellten Zuwachsraten an Adipösen nicht unbegrenzt fortschreibbar sind und zum anderen weitere Aspekte zum Tragen kommen können; immerhin werden - wie gezeigt - Anstrengungen zur Prävention und Vermeidung von Adipositas unternommen. Die Wirksamkeit und Nachhaltigkeit ergriffener Maßnahmen muss sich allerdings erst noch einstellen. Auch Ernährungsgewohnheiten und die Einstellung der Bevölkerung zu Gesundheitsaspekten können sich mit der Zeit verändern.

Aktuell wird v.a. seitens der WHO davon ausgegangen, dass nach wie vor eine Entwicklung vorherrscht, die zu deutlich steigenden Adipositaszahlen in den kommenden Jahren führen wird. Unter diesem Aspekt werden auch die anschlieBenden Szenarien durchgeführt. Sie sind dennoch weniger als zwingende Tendenzen aufzufassen, die unabwendbar zu sein scheinen, als vielmehr im Sinne konkreter Beispielrechnungen für jenen Fall zu verstehen, dass eine Trendumkehr hin zu sinkenden oder zumindest zu nicht weiter ansteigenden Adipositaszahlen in absehbarer Zeit nicht gelingen sollte. Ein ggf. interessierender Abgleich mit Ist-Daten kann erst ab dem Zeitpunkt deren Verfügbarkeit vorgenommen werden. Wie sich allerdings für die Ist-Daten dieser Analyse beispielhaft zeigt, ist die dafür erforderliche Datenbasis einerseits spärlich, andererseits erst mit beachtlicher zeitlicher Verzögerung zu erschließen, was einen zeitnahe Überprüfung der Hochrechnungen wesentlich erschwert bzw. unmöglich macht. 


\subsubsection{Szenario 1 unter der Annahme mäßiger Entwicklungen}

Dieses erste Szenario geht von der These aus, dass die Kostenbelastung in vergleichbarer Weise zunimmt wie die Prävalenz von Adipositas, ausgehend von einem berechneten Kostenniveau im (in Teilen virtuell definierten) Bezugsjahr 2003. Bei konstanten Zuwachsraten an adipösen Bevölkerungsanteilen in Gesamteuropa (i.S. der „Europäischen Region der WHO“) ${ }^{147}$ ist gemäß den Angaben der WHO damit zu rechnen, dass ausgehend von etwa 130 Mio. adipösen Erwachsenen (2005) im Jahr 2010 Europaweit bereits etwa 150 Mio. Erwachsene von Adipositas (BMI > 30) betroffen sein werden, was einer Zunahme von (aufgerundet) etwa 16\% in fünf Jahren bzw. einem Zuwachs von 4 Mio. Personen im Jahr entspricht. Frauen weisen in den WHO-Prognosen mit 2,4\% höhere Prävalenzsteigerungsraten auf als Männer, bei denen Raten von 2,2\% vorausgesagt werden, die für Deutschland im Vergleich zu anderen Staaten der Europäischen Region ggf. noch höher ausfallen könnten. ${ }^{148}$ Diese Prognosen werden den anschließenden Kostenberechnungen zugrunde gelegt. Ausgangspunkte sind dabei die epidemiologischen Daten für die Bundesrepublik Deutschland und die im Verlauf der Arbeit ermittelten Kosten. Bis 2010 werden nach WHO zudem etwa 15 Mio. adipöse Kinder in der gesamten Europäischen Region leben. Nach Prognosen der International Obesity Task Force werden bis zu diesem Zeitpunkt ca. 38\% der europäischen Kinder im schulpflichtigen Alter übergewichtig, mehr als ein Viertel davon sogar adipös sein. ${ }^{149}$ Die explizite Berücksichtigung von Personen unter 15 Jahren erfolgt aufgrund des gewählten Humankapitalansatzes zwar nicht, doch enthalten die hochgerechneten Zuwachsraten implizit jene Kinder, die im Verlauf der Betrachtungszeit die Schwelle von 15 Lebensjahren überschreiten, so dass sie zumindest in dieser Weise in die Kalkulationen einfließen.

\footnotetext{
${ }^{147}$ Die Europäische Region der WHO erstreckt sich über die Grenzen der Europäischen Union hinaus und schließt auch Länder ein, wie z.B. die Türkei, Israel, Armenien, die Russische Föderation u.a. und zeigt damit, dass die Prävalenzen von Adipositas auch in diesen Regionen bereits ein gesellschaftliches Problem darstellen. Vgl. WHO (2005).

${ }^{148}$ WHO (2005).

${ }^{149}$ Wang Y, Lobstein T. (2006).
} 
Mit dem prognostizierten Anstieg von Adipositas wird, aufgrund der in vorangegangenen Abschnitten erläuterten Zusammenhänge und Erwartungen, ein entsprechender Anstieg der Adipositastherapien (Operationen der Wahl; Ernährungsberatung, Bewegungstherapie) angenommen. Darüber hinaus werden die auf Adipositas zurückzuführenden Erkrankungshäufigkeiten - in erster Linie Diabetes mellitus Typ 2 - und damit verbundene Therapieanforderungen steigen, womit sich wiederum höhere attributable Kosten verbinden, die es abzuschätzen gilt. Mit Blick auf die indirekten Kosten sind neben häufigerer Arbeitsunfähigkeit und Invalidität auch gesteigerte Mortalitätsraten zu erwarten.

Tabelle 24: $\quad$ Szenario 1: Hochrechnung von Kosten für Adipositas bis ins Jahr 2020 mäßige Entwicklung

\begin{tabular}{|c|c|c|c|c|c|c|}
\hline Jahr & $\begin{array}{l}\text { Anzahl } \\
\text { adipöse } \\
\text { Persone } \\
\text { n }\end{array}$ & $\begin{array}{c}\text { Zunahme } \\
\text { Prävalenz } \\
\text { (WHO 2005) }\end{array}$ & $\begin{array}{l}\text { Wachstum } \\
\text { Gesundheit } \\
\text { (Rate BIP) }\end{array}$ & $\begin{array}{l}\text { Direkte } \\
\text { Kosten }\end{array}$ & $\begin{array}{l}\text { Indirekte Kosten } \\
\text { (nur } 4 \% \text { Diskont) }\end{array}$ & Gesamtkosten \\
\hline & $\ln 1.000$ & $\ln \%$ & $\ln \%$ & $\ln 1.000 €$ & $\ln 1.000 €$ & $\ln 1.000 €$ \\
\hline $2003^{*}$ & 13.200 & 0 & 0 & 11.350 .710 & 1.656 .275 & 13.006 .985 \\
\hline $2005^{* *}$ & 14.048 & 6,4 & 2,4 & 12.349 .572 & 1.802 .027 & 14.151.599 \\
\hline 2010 & 16.292 & 16 & 6 & 15.066 .478 & 2.198 .473 & 17.264 .951 \\
\hline 2015 & 18.898 & 16 & 6 & 18.381 .103 & 2.682 .137 & 21.063 .240 \\
\hline 2020 & 21.921 & 16 & 6 & 22.424 .946 & 3.272 .207 & 25.697 .153 \\
\hline \multicolumn{7}{|c|}{$\begin{array}{l}\text { "Basisjahr zur Kostenberechnung. } \\
\text { ** Kalkulierte Berechnung auf Grundlage des Basisjahres. Zum Abgleich der Hochrechnung mit } \\
\text { den tatsächlichen Kosten für das bereits zurückliegende Jahr } 2005 \text { (Ist-Werte) wäre-bei } \\
\text { Verfügbarkeit der relevanten Daten - eine erneute Berechnung der Gesamtkosten vorzunehmen } \\
\text { bzw. die vorliegende Berechnung fortzuschreiben. }\end{array}$} \\
\hline
\end{tabular}

Zusätzlich wird der Tatsache Folge geleistet, dass die allgemeinen Gesundheitskosten zumindest mit einer (moderat geschätzten) durchschnittlichen jährlichen Wachstumsrate des BIP in Höhe von 1,2\% einhergehen oder sogar darüber liegen. ${ }^{150}$ Dabei wird eine reale Zunahme zunächst bzgl. des Basisjahrs 2003

\footnotetext{
150 Die Wachstumsprognosen des BIP leiten sich aus den Analysen von Schlander, M. et al. (2004) ab, die ebenfalls unterschiedliche Szenarien für das reale Wachstum des BIP zugrundelegen und unter Berücksichtigung verschiedener Aspekte eine Rate von 1,2\% als
} 
vorausgesetzt (demnach bis 2005 2,4\%), danach werden vereinfachend die realen Zunahmen von jährlich 1,2\% im 5-Jahresrhythmus (also im Summe 6\%) jeweils bzgl. der Bezugsjahre 2005, 2010, 2015 unterstellt. Insofern wird auch für die Kosten der Adipositas eine allgemeine Steigerungsrate in dieser Höhe angenommen und auf Basis der Gegebenheiten des jeweiligen Bezugsjahres (also real) berechnet. Denkbar wäre z.B. auch eine Kopplung an die Steigerungsraten der GKV-Beiträge, die allerdings weniger den Marktanforderungen als vielmehr politischen Entscheidungen unterliegen. Zudem besteht eine Wachstumslücke beitragspflichtiger Einnahmen der GKV, welche trotz beachtlicher Steigerungsraten der Beiträge in der Vergangenheit nicht mit dem BIP-Wachstum schritthalten konnten. ${ }^{151}$

Die Ergebnisse unterstellen eine konstant steigende Prävalenzentwicklung adipöser Personen über die kommenden Jahre, die ausgehend von epidemiologisch ermittelten rd. 13,2 Mio. in 2003, bis ins Jahr 2010 um 23,4\% und bis ins Jahr 2015 um 43,1\% auf über 18,8 Mio. Betroffene anwachsen könnte. Damit wären unter der Annahme von proportionalen Verhältnissen Gesamtkosten in Höhe von 17,26 Mio. $€(2010)$ bzw. 21,06 Mio. $€(2015)$ verbunden. Berücksichtigt man zudem das jährliche reale Wachstum des BIP von im Durchschnitt 1,2\%, dann würde es im Jahr 2010 rund 2.315 Mrd. $€$ betragen und der darauf bezogene Anteil an Ausgaben für Adipositas beliefe sich auf 0,74\%. In 2015 läge er mit Bezug auf ein BIP in Höhe von 2.457 Mrd. $€$ bereits bei 0,86\%. Im Rahmen dieses Szenarios würde 2020 die Anzahl an adipösen Personen auf über 21 Mio. angestiegen sein und die Gesamtkosten beliefen sich auf rd. 25,7 Mrd. $€(0,98 \%$ von 2.608 Mrd. €). Ohne gegensteuernde Maßnahmen (z.B. Prävention) wäre bereits bei dieser moderaten Prognose das Gesundheitssystem wohl kaum dazu in der Lage, langfristig diesem Kostendruck standzuhalten.

geeignet ansehen. Dabei wird unterstellt, dass ggf. Jahre mit geringerem Wachstum durch Jahre mit höherem Wachstum ausgeglichen werden.

${ }^{151} \mathrm{Vgl}$. Schlander, M. et al. (2005), S. 9 und S. $15 \mathrm{ff}$. 


\subsubsection{Szenario 2 unter der Annahme progressiver Entwicklungen}

In einem zweiten Szenario wird von einem dynamisierten Zusammenhang zwischen der Entwicklung von Adipositas und den aufzubringenden Gesundheitskosten ausgegangen. Dies beruht v.a. auf der Annahme, dass zukünftig aufgrund früherer und häufigerer Krankheitsfolgen verstärkt multidisziplinäre Therapieverfahren und weiterer technologischer Fortschritt zur Anwendung kommen werden, die damit gemeinsam zwar der Komplexität von gesundheitlichen Folgen durch Adipositas gerecht würden, andererseits wohl aber auch deutlich höhere direkte Kosten verursachten. Gleichzeitig wird angenommen, dass - gemäß der im Rahmen der direkten Kostenermittlung gestellten Erwartungen ${ }^{152}$ - Einzeltherapien wie z.B. Magenband-OPs, Ernährungsberatungen in steigenden, überproportionalem Umfang durchgeführt und Medikamente häufiger bzw. intensiver zum Einsatz kommen werden. Auch ein möglicher systematischer Effekt sollte bedacht werden, nämlich dass mit steigendem Problem und Problembewusstsein auch die Diagnose „Adipositas“ ggf. häufiger als bisher gestellt werden könnte und dadurch unterrepräsentierte Kosten stärkere Berücksichtigung finden. Dies würde in der Konsequenz bedeuten, dass selbst unter Berücksichtigung unveränderter medizinischer Zusammenhänge, d.h. bei konstanter Einschätzung der Krankheitsfolgen von Adipositas und somit der beizumessenden Anteile, überdurchschnittliche Kostensteigerungen zu verzeichnen wären, die auf Grundlage der steigenden Prävalenzzahlen aus Szenario 1 angenommenen Verhältnisse hinaus gingen. Unterstellt man zudem steigende Entwicklungen der indirekten Kostendeterminanten, kann unter Beibehaltung der WHO-Prognosen bzgl. der Prävalenzen ein offensiveres Hochrechnungsszenario bis 2020 vorgenommen werden.

Zur konkreten Berechnung des Szenarios 2 stellt sich nun die Frage, in welcher Weise dieses zusätzlich erwartete Kostenwachstum in die Prognose einfließen könnte. Da bezüglich der Kostenentwicklung für Adipositas weder genauere Anhaltspunkte vorhanden sind, noch ein überzeugender Weg darin gesehen wird, zusätzliche unsichere Annahmen zugrunde zu legen, werden die in Szenario 1 zugrunde gelegten Prognosedaten modifiziert. Zum einen wird jetzt von einem

\footnotetext{
${ }^{152}$ Siehe Abschnitt 3.1.1.
} 
höheren jährlichen BIP-Wachstum von 1,8\% ausgegangen. ${ }^{153}$ Gleichzeitig folgt man der These, dass die Gesundheitsausgaben nicht mehr nur dem Umfang des BIP-Wachstums entsprechen, sondern noch um 2\%-Punkte darüber liegen und dadurch eine Finanzierungslücke aufweisen. ${ }^{154}$ Diese offensivere Annahme einer jährlichen Dynamisierung der Kosten in Höhe von 3,8\% wird auf die direkten und indirekten Kostenbeiträge von Adipositas bezogen und führt, unter ansonsten zu Szenario 1 unveränderten Rahmenbedingungen und gleicher realer Berechnungsweise, zu folgenden offensiven Prognosen:

Tabelle 25: $\quad$ Szenario 2: Hochrechnung von Kosten für Adipositas bis ins Jahr 2020 dynamisierte Entwicklung

\begin{tabular}{|c|c|c|c|c|c|c|}
\hline Jahr & $\begin{array}{c}\text { Anzahl } \\
\text { adipöse } \\
\text { Persone } \\
\mathbf{n}\end{array}$ & $\begin{array}{c}\text { Zunahme } \\
\text { Prävalenz }\end{array}$ & $\begin{array}{c}\text { Wachstum } \\
\text { (WHO 2005) }\end{array}$ & $\begin{array}{c}\text { Direkte } \\
\text { Gesundheit } \\
\text { (offensiv) }\end{array}$ & $\begin{array}{c}\text { Indirekte Kosten } \\
\text { (nur 4\% Diskont) }\end{array}$ & Gesamtkosten \\
\hline & In 1.000 & In \% & In \% & In 1.000€ & In $1.000 €$ & In $1.000 €$ \\
\hline $2003^{*}$ & 13.200 & 0 & 0 & 11.350 .710 & 1.656 .275 & 13.006 .985 \\
\hline $2005^{* *}$ & 14.048 & 6,4 & 7,6 & 12.939 .809 & 1.888 .153 & 14.827 .963 \\
\hline 2010 & 16.292 & 16 & 19 & 17.468 .742 & 2.549 .007 & 20.017 .750 \\
\hline 2015 & 18.898 & 16 & 19 & 23.582 .802 & 3.441 .159 & 27.023 .962 \\
\hline 2020 & 21.921 & 16 & 19 & 31.836 .783 & 4.645 .565 & 36.482 .349 \\
\hline
\end{tabular}

*Basisjahr zur Kostenberechnung.

** Kalkulierte Berechnung auf Grundlage des Basisjahres. Zum Abgleich der Hochrechnung mit den tatsächlichen Kosten für das bereits zurückliegende Jahr 2005 (Ist-Werte) wäre - bei Verfügbarkeit der relevanten Daten - eine erneute Berechnung der Gesamtkosten vorzunehmen bzw. die vorliegende Berechnung fortzuschreiben.

Quelle: Eigene Berechnung auf Grundlage der vorliegenden WHO-Daten.

Es zeigt sich, dass sich die Kosten unter diesen Annahmen bis zum Jahr 2020 bei konstant zunehmender Prävalenz von Adipositas nahezu verdreifachen könnten. Stellt man erneut die Relation zum BIP dieser Jahre her, dann beliefe sich die Gesamtausgabenquote im Jahr 2010 auf 0,83\% (BIP 2.413 Mrd. €), 2015 auf

\footnotetext{
${ }^{153}$ Die entspricht den Ergebnissen der „Rürup-Kommission“ auf die sich auch Schlander, M. et al., beziehen. Auch hier ist wieder unterstellt, dass Jahre mit geringerem BIP-Wachstum durch Jahre mit höherem Wachstum kompensiert werden.

${ }^{154}$ Schlander, M. et al. (2005), S. $20 \mathrm{ff}$.
} 
1,02\% (BIP 2.638 Mrd. €) und im Jahr 2020 schließlich auf einen gravierenden und überaus bedenklichen Anteil von über 1,26\% (BIP $2.884 \mathrm{Mrd} . €$ ).

In Anbetracht der bestehenden Unsicherheiten und Annahmen der Prognosen sind die Ergebnisse zweifellos kritisch zu betrachten. Je nach Einschätzung der Adipositas-Entwicklung ließen sich durchaus auch moderatere Szenarien darstellen und je nach Sichtweise ggf. rechtfertigen. Hier wurde sich den eher expansiven WHO-Prognosen angeschlossen und davon ausgehend Hochrechnungen vorgenommen, die einen denkbaren Beitrag auf einer kalkulierten Datengrundlage liefern. Die Ergebnisse repräsentieren - unter getroffenen modellhaften Annahmen - mögliche Ausmaße und Konsequenzen eines überaus präsenten und im Gesundheitswesen vielfach diskutierten Problembereichs. Im Diskussionsteil werden einzelne Aspekte dieser Berechnungen nochmals aufgegriffen.

\subsection{Internationaler Vergleich zu den USA und Großbritannien}

Ein internationaler Vergleich zu den USA und Großbritannien wirft erwartungsgemäß einige Schwierigkeiten auf. Auch wenn das prinzipielle Vorgehen mit der Klassifizierung von direkten und indirekten Kosten und die anteilsmäßige Einbeziehung von assoziierten Erkrankungen in den Vergleichsländern weitgehend identisch ist, bestehen dennoch erhebliche Untersuchungsspielräume, die zu systematischen Abweichungen bei den ermittelten Einzelergebnissen führen. Es zeigt sich beispielsweise bei repräsentativen Untersuchungen aus den USA, ${ }^{155}$ dass selbst bei Berücksichtigung eines vergleichbaren Studienpools, Unterschiede zum einen in der Auffassung über die tatsächliche Ausprägung von attributiven Risiken assoziierter Erkrankungen bestehen. Zum anderen spielt es eine wesentliche Rolle, ob eine eher konservative oder offensive Untersuchungsstrategie angesetzt wurde, ob zudem nach Grad der Adipositas bzw. nach Geschlecht unterschieden wurde und in welchem Umfang und Differenzierungsgrad die herangezogenen Erkrankungen berücksichtigt wurden. Um dies durchgängig zu klären, müsste ein detaillierter, methodisch umfassender Vergleich sowie ein Abgleich anhand der herangezogenen ICD 10-Diagnosen durchgeführt werden, was als sehr aufwendig anzusehen ist und nicht unter die Anforderungen dieser Arbeit fällt. Diese Situation führt dazu, dass sich v.a. die zugrunde gelegten 
beizumessenden Anteile teils wesentlich unterscheiden, ohne deswegen grundsätzlich ungeeignet oder gar falsch zu sein. Nachfolgende Tabelle zeigt die bestehenden Differenzen zu den USA in Bezug auf die wichtigsten identifizierten Diagnosen. Die attributiven Risiken für die USA werden dabei getrennt nach Geschlecht und obesity classes angegeben. Für Deutschland wurde eine vergleichbare differenzierte Aufschlüsselung der Risiken nicht realisiert, da für eine Kostenanalyse auf dieser Gliederungsebene nur bedingt adäquate Daten zur Verfügung stehen und dadurch ein erforderlicher expansiver Berechnungsaufwand im Missverhältnis zu einem kaum erzielbaren Mehrwert stünde: ${ }^{156}$

Tabelle 26: Unterschiede von bevölkerungsbezogenen beizumessenden Anteilen (PAF) zu Untersuchungen aus den USA und Deutschland.

\begin{tabular}{|c|c|c|c|c|c|}
\hline \multirow[t]{2}{*}{ Disease } & \multicolumn{2}{|c|}{$\begin{array}{l}\text { Mc Cusker et al.: } \\
\text { PAF in Men (\%) } \\
\text { for the USA }\end{array}$} & \multicolumn{2}{|c|}{$\begin{array}{l}\text { Mc Cusker et al.: } \\
\text { PAF in Women (\%) } \\
\text { for the USA }\end{array}$} & \multirow{2}{*}{$\begin{array}{l}\text { PAF (\%) für } \\
\text { Deutschland } \\
\text { Adipositas ab } \\
\text { BMI } \geq 30\end{array}$} \\
\hline & $\begin{array}{l}\text { Class I } \\
\text { Obesity }\end{array}$ & $\begin{array}{c}\text { Class II/III } \\
\text { Obesity }\end{array}$ & $\begin{array}{l}\text { Class I } \\
\text { Obesity }\end{array}$ & $\begin{array}{l}\text { Class II/III } \\
\text { Obesity }\end{array}$ & \\
\hline Diabetes mellitus & 21.2 & 22.9 & 21.2 & 31.5 & 42,5 \\
\hline Hypertonie & 15.7 & 9.6 & 12.0 & 13.4 & 9,7 \\
\hline Coronary heart disease & 13,4 & 6,6 & 5,9 & 7,3 & \multirow{2}{*}{5,5} \\
\hline Congestive heart failure & 10,4 & 8,0 & 5,1 & 21,2 & \\
\hline Stroke & 13,8 & 10,3 & -- & 1,6 & 3,7 \\
\hline Osteoarthritis & 16,7 & 10,9 & 14,1 & 19,7 & $9,1^{*}$ \\
\hline Colon-CA & 7,5 & 2,8 & 4,3 & 3,9 & 3,0 \\
\hline Prostate-CA & 4,6 & 1,1 & -- & -- & 1,6 \\
\hline $\begin{array}{l}\text { Endometrial-, Cervix-, } \\
\text { Ovarian-CA }\end{array}$ & -- & -- & 16,1 & 25,5 & 10,3 \\
\hline
\end{tabular}

${ }^{*}$ Arthrose

Quelle:

Eigene Zusammenstellung auf Grundlage der Darstellung Mc Cusker, M. E. et al. (2004), S. 31.

Betrachtet man daraufhin das Ausmaß der Kostenbelastung durch Übergewicht und Adipositas (overweight and obesity) in Relation zu den gesamten Gesundheitskosten der USA in Höhe von etwa 2.050 Mrd. \$ (OECD), wird der Anteil mit 5,7\% oder rd. 116,8 Mrd. \$ angegeben. ${ }^{157}$ Die gesamten direkten Kosten (2004) für Übergewicht und Adipositas (inkl. assoziierter Erkrankungen) werden je nach

\footnotetext{
${ }^{155}$ Vgl. Mc Cusker, M. E. et al. (2004).

${ }^{156} \mathrm{Vgl}$. ebenda, S. 31.ff

${ }^{157}$ Wolf. A. M. (2007).
} 
Untersuchungsbezug zwischen 65,9 Mrd. $\$^{158}$ und 71,7 Mrd. $\$^{159}$ beziffert. Die Differenz zu den Gesamtkosten ist mit den indirekten Kosten gleichzusetzen, die durchschnittlich etwa mit 48 Mrd. \$ zu beziffern sind. Aus Übersichtlichkeitsgründen wird hier in erster Linie ein Vergleich anhand der direkten Kosten durchgeführt. Deutlich mehr als die Hälfte der direkten Kosten werden durch Personen mit einem BMI $\geq 30$ erzeugt. Erwartungsgemäß stellen in dieser Gruppe, wie in Deutschland auch, Diabetes mellitus und KHK die größten Kostendeterminanten dar. Stellt man die ermittelten Kosten aus Deutschland jenen aus den USA gegenüber, so ergibt sich nachfolgende Tabelle. In der Aufstellung können nur Erkrankungen berücksichtigt werden, die zugleich in den Untersuchungen beider Seiten als relevant herangezogen wurden, so dass die sich daraus ergebenden relativen Anteile auch nur auf diese selektiven Krankheiten zu 100\% addieren. Dabei liegen für beide Nationen die identifizierten PAF aus obiger Tabelle 26 zugrunde:

Tabelle 27: Gegenüberstellung der direkten Kosten selektiver assoziierter Erkrankungen, deren relative Anteile zwischen den USA und Deutschland (Angaben in Mio. \$ bzw. € und \%) ${ }^{160}$

\begin{tabular}{|c|c|c|c|c|c|}
\hline \multirow[t]{2}{*}{$\begin{array}{l}\text { Erkrankungen } \\
\text { (selektiv) }\end{array}$} & \multicolumn{2}{|c|}{$\begin{array}{l}\text { Attributable } \\
\text { Kosten USA }\end{array}$} & \multirow{2}{*}{$\begin{array}{c}\text { Relative } \\
\text { Anteile } \\
\text { In \% }\end{array}$} & \multirow{2}{*}{$\begin{array}{l}\text { Attributable } \\
\text { Kosten } \\
\text { Deutschland } \\
\text { In Mio. } €\end{array}$} & \multirow{2}{*}{$\begin{array}{c}\text { Relative } \\
\text { Anteile } \\
\text { In } \%\end{array}$} \\
\hline & $\begin{array}{l}\text { In Mio. } \\
\text { US \$ }\end{array}$ & $\begin{array}{l}\text { In Mio. } \\
\in\end{array}$ & & & \\
\hline $\begin{array}{r}\text { Diabetes mellitus (v.a } \\
\text { Typ 2) }\end{array}$ & 13.505 & 8.684 & 29,7 & 6.814 & 76,8 \\
\hline Hypertension & 7.335 & 4.716 & 16,1 & 788 & 4,7 \\
\hline Coronary heart disease & 9.142 & 5.878 & 20,1 & \multirow{2}{*}{1.920} & \multirow{2}{*}{11,3} \\
\hline Congestive heart failure & 4.520 & 2.906 & 10,0 & & \\
\hline Stroke & 2.898 & 1.863 & 6,4 & 116 & 0,7 \\
\hline Osteoarthritis & 6.256 & 4.023 & 13,8 & 965 & 5,7 \\
\hline Kolon CA & 674 & 433 & 1,5 & 36 & 0,2 \\
\hline Prostata CA & 355 & 228 & 0,8 & 22 & 0,1 \\
\hline $\begin{array}{r}\text { CA } \text { } \\
\text { Geschlechtsorgane }\end{array}$ & 736 & 473 & 1,6 & 86 & 0,5 \\
\hline Summe & 45.421 & 29.204 & 100 & 10.747 & 100 \\
\hline
\end{tabular}

Quelle: $\quad$ Eigene Zusammenstellung auf Grundlage der Darstellung Mc Cusker, M. E. et al. (2004), S. 32

\footnotetext{
${ }^{158}$ Wolf, A. M. (2007).

${ }^{159}$ Mc Cusker, M. E. et al. (2004).

${ }^{160}$ Angesetzter Tageswechselkurs vom 20.05.2008: $1 \$=0,643 €$.
} 
Der dominanteste Unterschied in den Berechnungen zeigt sich über den zu berücksichtigenden Anteil der Kosten für Diabetes mellitus. Absolut gesehen liegen die Diabeteskosten mit $13.505 \$(8.648 €)$ in den USA um etwa 1,8 Mrd. $€$ höher als jene in Deutschland. Relativ betrachtet weist Deutschland einen Anteil von knapp $77 \%$ an den herangezogenen Erkrankungskosten auf, während für die USA lediglich rd. 30\% zu Buche stehen. Hier spielt eine wesentliche Rolle, dass durch das Ausklammern des Aspekts "Übergewicht" bei den Daten aus den USA, rund 40\%-Punkte der Kosten herausgerechnet wurden. Übergewicht und Adipositas zusammen repräsentieren bezogen auf die gesamten direkten Kosten des Landes einen relativen Anteil von über 70\% (rd. $51 \mathrm{Mrd}$. \$). Andererseits ist bemerkenswert, dass die Kosten für Hypertonie in den USA im Rahmen der betrachteten Erkrankungen einen um mehr als das 3-fache höheren relativen Anteil aufweisen als in Deutschland, obwohl die Abweichungen der beizumessenden Anteile hierbei nicht gravierend sind.

Eine genaue Untersuchung beispielsweise der diesbezüglichen Arztbesuche bzw. des Medikamentenverbrauchs zur Behandlung der Hypertonie könnte ggf. zu weiterem Aufschluss beitragen. Die Herzerkrankungen spielen in den USA ebenfalls eine bedeutende Rolle und werden um etwa 3,5-fach höher beziffert als in Deutschland. In Bezug auf Schlaganfälle ist eine deutlich höhere Kostenbelastung auf Seiten der USA festzustellen, ebenfalls bzgl. einer Osteoarthritis (hier allerdings der ungenaue Vergleich zur Diagnose "Arthrose" in Deutschland). Die Krebserkrankungen haben aus beiden Seiten betrachtet insgesamt einen eher untergeordneten Stellenwert.

Auch die Erhebungen zur Kostenbelastung durch Adipositas in Großbritannien weisen hinsichtlich angewendeter Methoden und Umfang der Schätzungen eine erhebliche Variationsbreite auf. 
Tabelle 28: Direkte Kosten selektiver assoziierter Erkrankungen ungewichtet und gewichtet und relative Anteile für Großbritannien (Angaben in Mio. $£$ bzw. € und \%) 161

\begin{tabular}{|l|c|c|c|c|c|c|}
\hline Disease categories & $\begin{array}{c}\text { Kosten für das } \\
\text { NHS (ungewichtet) }\end{array}$ & PAF & $\begin{array}{c}\text { Attributale Kosten } \\
\text { Übergewicht und } \\
\text { Adipositas }\end{array}$ & $\begin{array}{c}\text { Relative } \\
\text { Anteile }\end{array}$ \\
\hline & In Mio. $\mathbf{E}$ & In Mio. $€$ & In \% & In Mio. $\mathbf{~ I n ~ M i o . ~} €$ & In \% \\
\hline Type 2 Diabetes & 700 & 879 & 75,7 & 530 & 665 & 16,5 \\
\hline Hypertension & 1.000 & 1.256 & 58,0 & 580 & 728 & 18,1 \\
\hline Ischaemic heart disease & 2.300 & 2.888 & 33,9 & 780 & 979 & 24,3 \\
\hline Stroke & 2.900 & 3.642 & 33,8 & 980 & 1.230 & 30,6 \\
\hline Osteoarthritis & 1.100 & 1.381 & 20,9 & 230 & 289 & 7,1 \\
\hline Colon/ rectum CA & 400 & 502 & 15,0 & 60 & 75 & 1,8 \\
\hline CA q (Corpus uteri / breast) & 300 & 376 & 26,7 & 80 & 100 & 2,5 \\
\hline Summe & $\mathbf{8 . 7 0 0}$ & 10.927 & -- & $\mathbf{3 . 2 0 0}$ & 4.066 & $\mathbf{1 0 0}$ \\
\hline
\end{tabular}

Quelle:

Allender S./ Rayner M. (2006). The burden of obesity-related ill health.

Eine der wenigen verfügbaren und v.a. aktuellen Erhebungen gibt direkte Kosten in Höhe von 3.2 Mrd. £, allerdings für Übergewicht und Adipositas, zusammen an. ${ }^{162}$ Eine getrennte Betrachtung von Adipositas und Übergewicht auf Ebene einzelner Krankheitskategorien war nicht zu ermitteln, so dass eine Vergleichbarkeit nur bedingt gegeben ist. Im Rahmen der Erhebung wird zudem auf andere Schätzungen mit direkten Kosten zwischen 480 Mio. £ und £1.1 Mrd. in 2004 verwiesen. Die Untersuchung berücksichtigt lediglich 7 assoziierte Erkrankungen und stellt sich wie in obiger Tabelle zusammengefasst dar.

Mit dem höchsten ermittelten Wert von 3,2 Mrd. £ oder 4,06 Mrd. € liegen die Kosten in Großbritannien - sogar für Übergewicht und Adipositas zusammen dennoch deutlich hinter den Kosten in Deutschland und den USA zurück. Auch wenn die Bevölkerungszahlen in Großbritannien geringer sind, so hat sich doch im Zuge der epidemiologischen Zusammenhänge gezeigt, dass mit Blick auf Übergewicht und Adipositas ähnliche Relationen herrschen. Insofern verwundert v.a. die vergleichsweise geringen Kosten für Diabetes mellitus, die in der Relation zu den übrigen betrachteten Erkrankungen gerade einmal 16,5\% ausmachen und

${ }^{161}$ Angesetzter Tageswechselkurs vom 20.05.2008: $1 £=1,256 €$. 
deutlich unterrepräsentiert erscheinen. Mit einem Anteil von 30\% liegen die Schlaganfälle an erster Stelle der betrachteten Kosten. Es ist im Rahmen dieser Arbeit nicht zu klären, welche Bezugsdaten für diese Darstellung herangezogen wurden.

Generell macht der internationale Vergleich deutlich, dass offensichtliche Anforderungen für umfassendere Kostenstudien v.a. in Großbritannien bestehen. Eine internationale Vergleichbarkeit ist in erster Linie auf eine Standardisierung der Auswertungsgrundlagen und auf die Verfügbarkeit umfassender Einzelstudien angewiesen, so dass die Vergleiche nur eingeschränkte Aussagekraft besitzen und diesbezüglich weiterer Handlungsbedarf gesehen wird.

${ }^{162}$ Allender, S./ Rayner, M. (2007). 


\section{Diskussion}

Die Analyse wurde mit der Zielsetzung durchgeführt, die gegenwärtig bzw. zukünftig zu erwartenden Gesamtkosten von Adipositas und die diesbezüglichen Belastungen des Gesundheitssystems Deutschland einschätzen und quantifizieren zu können. Es wurden je nach zugrunde gelegtem Diskontierungssatz - Gesamtkosten von rd. 13,007 Mrd. € bzw. 12,754 Mrd. €, ermittelt, was Anteilen von 0,61\% bzw. 0,60\% am BIP des Jahres 2003 (2.130 Mrd. €) entspricht. Damit verbinden sich in der Konsequenz gesundheitspolitische Einschätzungen, die ggf. Allokations-, Distributions- oder Rationierungsmaßnahmen nach sich ziehen werden. An die Erhebung wurden aufgrund einer Mehrdimensionalität der einzubeziehenden Kostenbestandteile und der Existenz unterschiedlicher, uneinheitlicher Methoden und Ermessensspielräume besondere Anforderungen gestellt. Das Bestreben lag darin, eine umfassende, strukturierte und repräsentative Arbeit zu erstellen, die zu transparenten und somit verifizierbaren Resultaten kommt.

Aus diesen Gründen ist es erforderlich, die getroffenen Annahmen, Zusammenhänge und erzielten Ergebnisse der Arbeit kritisch zu diskutieren, Problemfelder zu identifizieren sowie Grenzen der Kostenanalyse abzustecken. Sowohl das Datenmaterial, die verwendete Methodik, epidemiologische Aspekte, die Selektion jener Erkrankungen, die in mittelbarem Zusammenhang zu Adipositas stehen und nicht zuletzt die ermittelten Kosten selbst, die darauf aufbauenden Hochrechnungsszenarien sowie die Vergleiche mit nationalen und internationalen Berechnungen sollen abschließend differenziert betrachtet werden.

\subsection{Methodische Abgrenzung}

Grundsätzlich ist voranzustellen, dass Gesamtkostenanalysen auf der Makroebene eines gesamten Landes in der Größenordnung wie Deutschland oder auch den USA, neben einem unbestrittenen Realitätsgehalt in bestimmten Teilen gleichzeitig einen Modellcharakter aufweisen und demnach in den Bereich der experimentellen Gesundheitsberichterstattung einzuordnen sind. ${ }^{163}$ Dies hängt u.a. damit zusammen, dass nicht alle Gesundheitskosten in benötigtem Umfang monetär bewertet vorliegen und wesentliche Anteile aus unterschiedlichen Zusammenhängen unter zu treffenden, teils 
restriktiven Annahmen erst berechnet bzw. abgeschätzt werden müssen, wie beispielsweise die gesamten indirekten Kosten aber auch Teile der direkten Kosten, denkt man z.B. an die nur wenig transparente Datensituation für operative Eingriffe bei Adipositas oder ambulante Aspekte wie Ernährungsberatung. Die Nichtberücksichtigung einiger Kostenanteile, wie z.B. die komplexe monetäre Bewertung von Einbußen der Lebensqualität (intangible Kosten), war im Rahmen der verwendeten Methodik des Humankapital-Ansatzes durchaus vertretbar und ist auch in Referenzstudien üblich. ${ }^{164}$ Nichtsdestotrotz darf dabei nicht verkannt werden, dass z.B. durch psychosoziale Aspekte wie Schmerz, Trauer, Stigmatisierung durchaus Produktivitätseinbußen und damit volkswirtschaftliche Verluste sehr wahrscheinlich sind, deren Messbarkeit jedoch eine nahezu unlösbare Aufgabe zu sein scheint. ${ }^{165}$ Insgesamt repräsentiert das methodische Vorgehen einen etablierten Standard, der in Kostenanalysen/ Metastudien dieser Ausprägung üblicherweise angewendet wird.

\subsection{Aspekte der Datenerhebung}

Eine zentrale Problematik und besondere Schwierigkeit bestand darin, die benötigten Ausgangsdaten nur bedingt in einer Weise vorzufinden, wie sie für eine Gesamtkostenbetrachtung benötigt würden. Grundsätzlich sollten alle nach Einzeldiagnosen aufgeschlüsselten direkten und indirekten Kosten ermittelt werden, die der Adipositas zuzurechnen sind. Im Zuge der Recherchen wurde offensichtlich, dass die offiziell verfügbaren Gesundheitskosten systematische Defizite aufweisen und erhebliche Zweifel an der Repräsentativität v.a. jener direkten Krankheitskosten der Adipositas nähren, welche den assoziierten Erkrankungen zuzuschreiben sind. $^{166}$ Über die durchgeführten eigenen Berechnungen auf Basis der herangezogenen Einzelstudien wurde diese These bestärkt und es wurden nahezu ausnahmslos höhere Kosten aus den Einzelstudien ermittelt. Zwar aufwendig aber in der Konsequenz durchaus sinnvoll wäre es, die Systematik der Datengewinnung und -erfassung offizieller Gesundheitsstatistiken stärker an den sich stellenden wachsenden Anforderungen des Themenbereichs Adipositas (und ggf. weiterer Themenbereiche, wie z.B. Rauchen, Alkoholabusus)

\footnotetext{
163 Henke, K.-D. (1997).

${ }^{164}$ Vgl. v.a. Kohlmeier, L. et al. (1993).

${ }^{165}$ Henke, K.-D. et al. (1986).

${ }^{166}$ Vgl. Hauner, H. et al. (2007).
} 
auszurichten. Aus Kostensicht liegt daher konsequenter Weise die Hauptforderung nahe, Adipositas nicht nur unter medizinischen, sondern auch unter statistischen Gesichtspunkten als komplexere und in erster Linie eigenständigere Diagnose aufzufassen. An einigen Stellen der Arbeit wurden Defizite bezüglich der Datenverfügbarkeit offen gelegt.

\subsection{Ermittlung direkter Kosten von Adipositas per se}

Zur Ermittlung der direkten Kosten von Adipositas (ohne Begleiterkrankungen) konnten aufgrund fehlender Routinestatistik weder exakte Zahlenangaben über stationäre Behandlungen und Operationshäufigkeiten gefunden werden, noch lagen umfassendere Erhebungen über Ausmaß und Umfang der ambulanten Behandlungen von Adipositas vor. Dies spricht möglicherweise für eine geringe Wahrnehmung einer isolierten Adipositasbehandlung als solches oder bestätigt die Auffassung, dass in der täglichen Praxis keine exakte Abgrenzung von Kosten vorgenommen wird und wesentliche Teile der Adipositasbehandlung anderen Erkrankungen (demnach anderen ICD 10-Ziffern) zugeschrieben werden. Die Problematik liegt in der Tatsache, dass Adipositas nach SGB keine Krankheit repräsentiert. Aus diesem Grund sind die Kosten der zur Verfügung stehenden Adipositas-Therapien durch die Krankenkassen i.d.R. nicht erstattungsfähig. Therapien/ Diagnosen werden möglicherweise unter den Ziffern v.a. jener Erkrankungen subsummiert, die als Folge von Adipositas gesehen werden, nicht zuletzt um eine Finanzierbarkeit für die Patienten zu ermöglichen. Kommt dieses Verhalten zur Anwendung, verbindet sich damit in der Konsequenz eine systematischen Unterschätzung der eigentlichen Diagnose Adipositas. Darüber hinaus muss in vielen Fällen davon ausgegangen werden, dass bei einer Reihe von Patienten-Untersuchungen das Vorliegen von Adipositas entweder unbeachtet bleibt oder falsch eingeschätzt wird und in vielen Fällen keine genauen Gewichts- und Körpergrößenbestimmungen vorgenommen werden oder aber durchgeführte Schätzungen/ Messungen häufig nicht zu einer im Sinne von ICD 10 dokumentierten Diagnose „Adipositas“ führen, die in die Statistik einfließen könnten. ${ }^{167}$

Aus diesen Gründen stützen sich die durchgeführten Berechnungen der direkten Kosten auf nur wenige verfügbare Einzelstudien und es ist auch hier davon auszugehen, dass die 
„wahren“ direkten Kosten der Adipositas deutlich höher liegen. Die Ermittlung der Medikamentenkosten wurde zudem dadurch erschwert, dass nur Einkauf- und keine Verordnungszahlen der zugelassenen Präparate zur Verfügung standen, da sie i.d.R. nicht von den Krankenkassen erstattet werden und somit nur bedingt statistisch erfasst werden. Zudem werden aktuelle, repräsentative Vertriebszahlen der Hersteller aus Wettbewerbsgründen nicht offenbart. Trotz nicht auszuräumender Zweifel an der Unvollständigkeit dieses Kostenteils der Untersuchung und der gleichzeitig kaum nachvollziehbaren deutlichen Abweichungen zu den diesbezüglichen Top-downVergleichsstudien, ist davon abgesehen worden, etwaige "Korrekturgrößen“ mit spekulativem Charakter einzuführen, da für sie zum einen keine fundierte Grundlage gesehen wurde. Zum anderen stellte sich die Frage, welche objektivierbaren Größen dafür überhaupt in Frage kämen. Auch auf diese Frage muss man eine Antwort schuldig bleiben.

Nichtsdestotrotz bleibt ungeklärt, welche Gründe tatsächlich für die teils erheblichen Differenzen der eigenen Berechnungen zu den Vergleichserhebungen mit deutlich höheren direkten Adipositas-Kosten verantwortlich zu machen sind. Neben der begrenzten Verfügbarkeit von Basisdaten ist anzunehmen, dass entweder ein wesentlicher Teil von Kosten in diese Erhebungen einberechnet wurden, die in den eigenen Berechnungen nicht berücksichtigt wurden (z.B. Kosten für Verwaltung und Personal, Gruppentherapien, Selbsthilfeprogramme, Hilfsmittel und Kosten für Medikamente/ Ernährungszusatzprodukte) oder aber seitens der Vergleichsstudien eine klare Abgrenzung zu den assoziierten Erkrankungen nicht konsequent eingehalten werden konnte. Gerade auf diese wurde bei den eigenen Berechnungen dagegen strikt geachtet. Ein Aspekt, welcher dazu beiträgt, die Bedeutung der herrschenden Differenzen dieses Untersuchungsbereichs zu relativieren liegt darin, dass dieser Kostenanteil im Vergleich zu den Kosten für assoziierte Erkrankungen eine statistisch nicht-signifikante Rolle einnimmt, so dass Defizite eher in Kauf zu nehmen sind. Dennoch bleibt der Klärungsbedarf der offensichtlichen Abweichungen der Ergebnisse weiterhin bestehen. Nicht zuletzt aus dieser Erkenntnis heraus sollte Sorge dafür getragen werden, dass durch systematische

\footnotetext{
167 Diese Aussagen wurden im Rahmen eines Interviews mit der Leitenden Oberärztin für AdipositasChirurgie der Universitätsklinik Ulm, Dr. Anna-Maria Wolf, am 21.05.2008, getroffen.
} 
Datenerhebung alle Kostenanteile gleichermaßen transparent zur Verfügung stehen und zweifelsfrei verarbeitet und präsentiert werden können.

\subsection{Ermittlung der Kosten assoziierter Erkrankungen von Adipositas}

Auf Grundlage medizinisch-epidemiologischer Untersuchungen ist den assoziierten Erkrankungen von Adipositas und somit auch den dadurch entstehenden Kosten ein besonderer Stellenwert zuzuschreiben. Einerseits repräsentieren sie jene Anteile, die der Adipositas im Hinblick auf die Entstehung und Progression aus medizinischer Sicht zugesprochen werden. Andererseits tragen sie implizit aber auch zur "statistischen Korrektur" der als unzureichend vermuteten Kostenabgrenzung zu jenen Erkrankungen bei, die zwar aufgrund von Adipositas begünstigt, jedoch im Zuge dessen nicht oder nur unzureichend erfasst werden. Dies kann dauerhaft jedoch weder Sinne einer objektiven Bewertung der Krankheitskosten, noch einer verursachungsgerechten Kostenzuteilung sein. Insofern ist auch aus diesen Gründen eine systematischere und klarer abgrenzbare Erfassung bereits bei der Entstehung dieser Kosten zu fordern.

Hinzu kommt, dass die absoluten bzw. die relativen Anteile an direkten Kosten der identifizierten Erkrankungen, welche mit Adipositas in kausale Verbindung gebracht werden (AHT, Diabetes mellitus, Depression usw.), statistisch nicht berücksichtigt und somit auch nicht gesondert erfasst werden, jedoch für eine Gesamtkostenbetrachtung zwingend erforderlich sind. Sie müssen grundsätzlich in weiteren Berechnungen aus den erfassten Kosten assoziierter Erkrankungen ermittelt werden. Im Zuge der Berechnungen wurden Einzelstudien aus dem In- und Ausland herangezogen, denen i.d.R. umfassende Bottom-up-Erhebungen zugrunde lagen. Es sollte bei allem Bestreben einer umfassenden Kostendarstellung jedoch gleichzeitig darauf geachtet werden, keine ggf. unangemessene Überbewertung von Adipositas vorzunehmen, etwa durch die anteilige Einbeziehung und Kostenzuweisung jener Zusammenhänge, die zwar als relevant anzunehmen sind, gegenwärtig jedoch auf noch unvollständig fundierten medizinischen Erkenntnissen beruhen (z.B. die durch Adipositas begünstigende Entstehung bestimmter Karzinome oder auch komplexere Kausalketten bestimmter Krankheitsverläufe). Durch ein als eher konservativ einzuschätzendes, selektives Vorgehen wurde dieser Anforderung nachgekommen. 
Die den assoziierten Erkrankungen zugrunde gelegten Einzelstudien wurden durch Recherchen in den einschlägigen Fachdatenbanken ermittelt. Bei den erzielten Ergebnissen ist $\mathrm{zu}$ bedenken, dass die teils auch ausländischen Studien auf unterschiedlichen Methoden und Annahmen oder auch Grundgesamtheiten beruhen und zum Teil verschiedene Jahre repräsentieren, die in der Konsequenz zu einem „virtuellen Bezugsjahr" führten. Insgesamt betrachtet konnten wesentliche Kostenanteile auf das Jahr 2003 bezogen werden, so dass die Gesamtergebnisse in erster Linie als repräsentativ für diesen Zeitraum zu betrachten sind. Zu kritisieren ist, dass innerhalb ggf. komplexerer Krankheitsgruppen (z.B. Ischämische Herzerkrankungen, Arthrose) unterschiedliche ICD10 Ziffernbereiche herangezogen wurden und diesbezüglich keine exakte Deckungsgleichheit z.B. zu den Vergleichsberechnungen des Bundes bestand. Dies gilt es auch mit Blick auf die Verwendung der beizumessenden Anteile zu bedenken. Hinzu kommt das Problem, dass für einige Krankheiten keine repräsentativen Kostenstudien ausfindig zu machen waren, was v.a. mit Blick auf Volkskrankheiten wie Hypertonie oder bösartige Neubildungen des Dickdarms sehr verwunderte und dahingehenden Handlungsbedarf verdeutlicht. In diesen Fällen wurde mangels Alternativen auf die Gesundheitsrechnung des Bundes verwiesen. Die verwendeten Einzelstudien zeigen bis auf eine Ausnahme (Depressionen) alle recherchierten Einzelstudien im Vergleich zu den Top-down-Berechnungen signifikant höhere Kosten. Hier haben - anders als im Falle der Berechnung der direkten Kosten für Adipositas per se - fundiertere Daten zur Verfügung gestanden, die dem gesamten Umfang und der Komplexität der jeweiligen Erkrankung gerecht werden konnten.

Die Gesamtübersicht aller durchgeführten Erhebungen bzw. Vergleichserhebungen zu den direkten Kosten assoziierter Erkrankungen von Adipositas zeigt die deutliche Diskrepanz zwischen den existierenden Kostenberechnungen und dem durchgeführten eigenen Berechnungsansatz. 
Abbildung 10: Direkte Kosten assoziierter Erkrankungen der Adipositas in Deutschland im Vergleich verschiedener Berechnungen

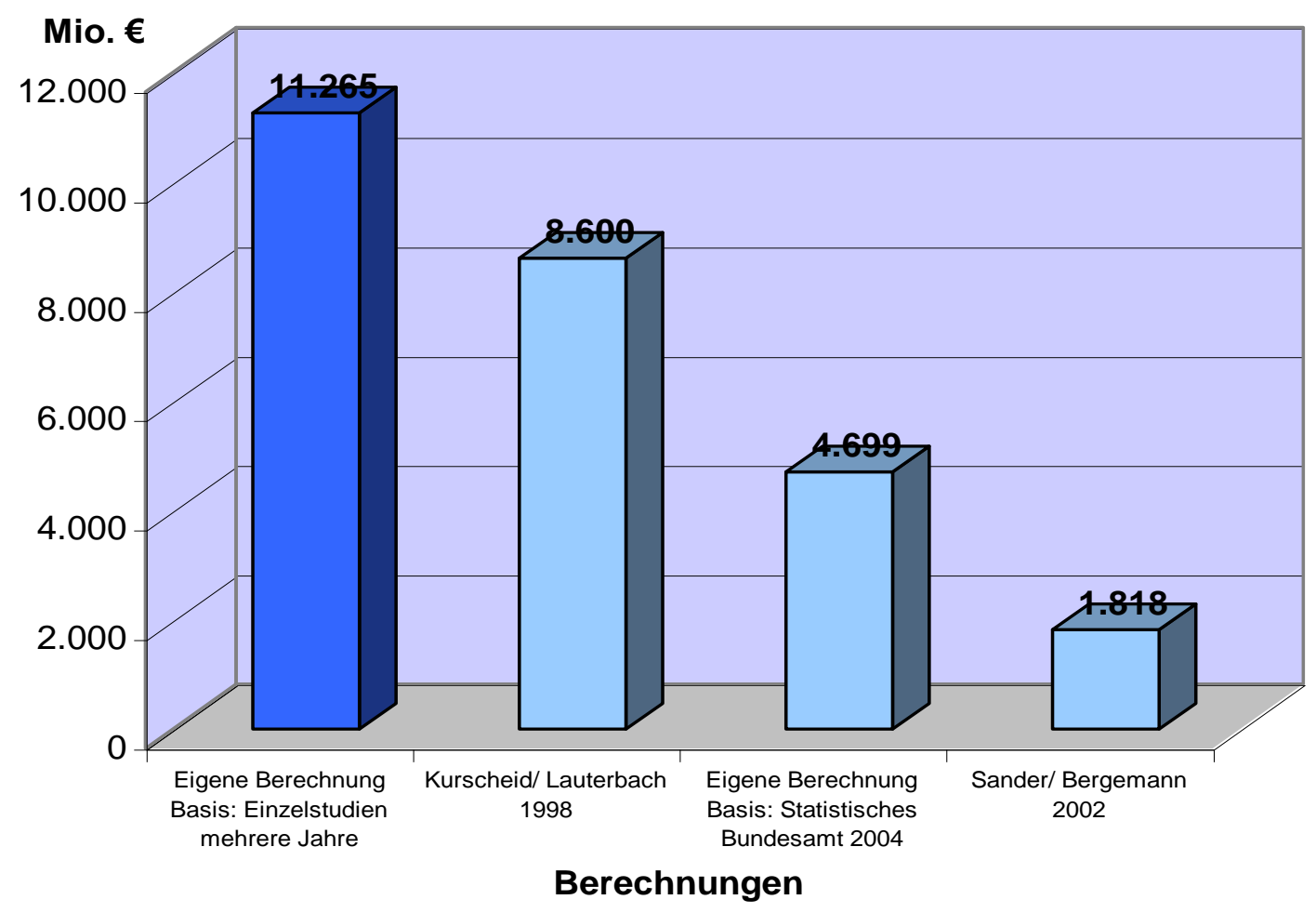

Quelle: $\quad$ Eigene Zusammenstellung.

Da die Vergleichsberechnungen durchweg Top-down-Ansätze repräsentieren, werden die Bedenken erhärtet, dass derartige Berechnungen durchgängig zu niedrige Kosten für Folge- und Begleiterkrankungen von Adipositas ansetzen. Die anteilig ermittelten Kosten für Diabetes mellitus in Höhe von 6,8 Mrd. $€^{168}$ würden allein bereits ausreichen, um - mit Ausnahme der Erhebung von Kurscheid/ Lauterbach - über den gesamten direkten Kosten der Vergleichsberechnungen zu liegen.

Methodische Besonderheiten der zugrunde gelegten Einzelstudien konnten nicht eingehender betrachtet werden. Allerdings war festzustellen, dass nicht durchgängig eine exakte Abgrenzung von Krankheitskosten in Bezug auf die entsprechenden ICD 10-Ziffern eingehalten wurde. Trotz bedingter methodischer Uneinheitlichkeit zeigt sich dennoch ein deutlicher Trend zu niedrigeren Kosten in allen Vergleichserhebungen, die Anteile

\footnotetext{
${ }^{168}$ Köster l. et al. (2006), S. 1498-1504.
} 
zwischen maximal 76\% (Kurscheid/ Lauterbach), 41\% (Basis: Statistisches Bundesamt) und gerade einmal 16\% (Sander/ Bergemann) bezogen auf die durchgeführten eigenen Berechnungen aufweisen.

\subsection{Ermittlung indirekter Kosten und beizumessender Anteile}

Die Ermittlung der indirekten Kostenanteile war anhand von monetär bewerteten Ausfallzeiten der von Krankheitsfolgen durch Adipositas betroffenen Patienten vorzunehmen. Die statistischen Schwierigkeiten stellten sich hierbei deutlich geringer dar als im Falle der Ermittlung der direkten Kosten, da von Seiten des Statistischen Bundesamtes, der AOK und des Verbands deutscher Rentenversicherer eine diesbezüglich vollständige und solide Datenbasis vorgefunden wurde. Es sind daher weniger die komplexen, mehrstufigen Berechnungsschritte im Rahmen des gewählten Humankapital-Ansatzes als vielmehr die damit verbundenen, üblichen restriktiven Annahmen, die zur Kritik an dem Vorgehen führen können. Dennoch hat sich bislang keine ggf. optimiertere Methodik durchgesetzt, so dass die Verwendung als gängige und vergleichbare Praxis anzusehen ist.

Die Wahl der Diskontierungssätze zur Berechnung der indirekten Kosten von Mortalität und Invalidität ist ebenfalls anzusprechen. Mit $4 \%$ und $6 \%$ wurden bislang üblicherweise verwendete Größenordnungen anderer Studien herangezogen, so dass in einer sonst statischen Betrachtung zumindest zwei Alternativszenarien zur Verfügung stehen. ${ }^{169}$ Diese Auswahl ist nicht unstrittig und es werden in der Literatur durchaus auch niedrigere oder teils sogar negative Diskontierungsraten gefordert, da ansonsten die Gefahr bestehen könnte, zukünftige Gesundheit und Gewinne aufgrund von Prävention in ggf. ungerechtfertigter Weise abzuwerten. ${ }^{170}$ Zugleich wird aber auch die Notwendigkeit zu weiteren Diskussionen zu diesem Thema eingeräumt. Im Rahmen dieser Arbeit wurde die Grundsatzfrage über die geeignete Höhe des Diskontierungssatzes der methodischen Vergleichbarkeit untergeordnet.

Ein weiterer Diskussionspunkt verbindet sich mit der Auswahl der beizumessenden Anteile für die Folge- und Begleiterkrankungen von Adipositas. Da bedauerlicherweise keine

\footnotetext{
${ }^{169}$ Vgl. in erster Linie Kohlemeier, L. et al (1993).
} 
international einheitlichen Anteile, z.B. seitens der WHO, vorliegen, wurde zur Zuweisung der als geeignet anzusehenden Werte auf bereits näher untersuchte beizumessende Anteile aus Erhebungen zurückgegriffen, die als Grundlage für vergleichbare Berechnungen der Schweiz verwendet und als überaus verlässlich eingeschätzt werden. ${ }^{171}$ Ein weiterer Grund für dieses Vorgehen ist in der Tatsache zu sehen, dass mit der Schweizer Studie die einzige von den Anforderungen vergleichbare und zugleich aktuelle Kostenstudie zu Adipositas im deutschsprachigen Raum vorgefunden wurde, die an unterschiedlichen Stellen zu Vergleichszwecken herangezogen werden konnte. Allerdings hat sich gerade beim Vergleich mit den USA und Großbritannien gezeigt, dass durchaus relevante Unterschiede in der Auffassung und Höhe der beizumessenden Anteile bestehen, die zu Problemen bei der Vergleichbarkeit von Ergebnissen führten, welche jedoch ohne genauere Kenntnis über das Zustandekommen nicht weiter zu beurteilen sind. Im Rahmen dieser Arbeit musste auf diesbezüglich weiterführende Untersuchungen verzichtet werden. Unter Berücksichtigung zahlreicher Einflussfaktoren wäre hierzu umfassendes Untersuchungspotenzial für eigenständige Studien gegeben, deren Ergebnisse idealer Weise zu standardisierten Werten führen könnten.

\subsection{Betrachtung der Gesamtkosten}

Geht man im nächsten Diskussionsaspekt auf die erzielten Gesamtkosten der Arbeit in Höhe von 13,007 Mrd. $€$ (Diskontierungsrate 4\%) bzw. 12,754 Mrd. $€$ (Diskontierungsrate $6 \%)$ ein, so ist zu berücksichtigen, dass neben den Kosten der Adipositas als solches, Anteile von 11 assoziierten Erkrankungen herangezogen wurden. Dass nicht noch weitere im Zusammenhang mit Adipositas als ursächlich vermutete Erkrankungen berücksichtigt wurden, hat zum einen mit dem bereits erwähnten Aspekt der Unsicherheit bezüglich einer eindeutig belegbaren Krankheitsrelevanz zu tun. Zum anderen spielt auch hier die Datenverfügbarkeit eine Rolle, so dass z.B. abgrenzbare Kosten im Zusammenhang mit Hyperlipidämien weder in Einzelstudien noch über die Gesundheitskostenrechnung des Bundes zu ermitteln waren. Insofern wurden diese Kosten zwar als implizite Bestandteile im Rahmen der Gefäßerkrankungen aufgefasst, tauchen aber nicht explizit auf. Ob daraufhin die Ergebnisse möglicherweise als zu gering $z u$ bewerten sind, liegt im Expertenermessen des fachkundigen Lesers. Insgesamt wurde sicherlich die eher konser-

${ }^{170}$ Henke, K.-D. et al. (1997). 
vativere Herangehensweise verfolgt, was auch dadurch belegt werden kann, dass lediglich unmittelbare Krankheitsfolgen in Verbindung mit Adipositas berücksichtigt (z.B. Diabetes mellitus) wurden und darüber hinaus z.B. keine zweit- oder drittgradigen Verknüpfungen (z.B. Schlaganfall aufgrund von Hypertonie aufgrund von Adipositas).

Zudem wird durch die Anwendung des Humankapital-Ansatzes selbst eine konservative Tendenz v.a. dadurch vorgegeben, dass - mit Blick auf die indirekten Kostenanteile lediglich 15-65-jährige Personen Eingang in die Berechnungen finden. Im Falle von Kindern kann dies als noch wenig relevant eingeschätzt werden, da sie einerseits noch nicht dem Arbeitsmarkt angehören, andererseits in jungen Jahren (zumindest gegenwärtig) noch keine erhöhten Mortalitätsrisiken durch Adipositas aufweisen und sie Kosten vornehmlich als adipöse Erwachsene generieren werden. Bedenkt man allerdings die je nach Altersgruppe langen Zeiträume, auf die sich die indirekten Kostenberechnungen beziehen, so ist z.B. damit zu rechnen, dass zukünftig ein deutlich höheres Erwerbsalter als heute gelten dürfte (ggf. 67 oder 70 Jahre) und zudem wachsende Prävalenzen von Adipositas (und ggf. der Begleiterkrankungen) eine wesentliche Rolle spielen werden, die unberücksichtigt bleiben. Da annahmegemäß alle Parameter ausgehend von der Gegenwart konstant gehalten werden, kommen derartige Effekte nicht zum Tragen, genauso wenig wie Einkommens- oder Risikoprofilveränderungen bzw. ein Wandel im Konsum- oder Ernährungsverhalten der Bevölkerung, was z.B. im Rahmen von Präventivinitiativen, wie der Nationalen Verzehrsstudie II, untersucht wird. Weitergehende Überlegungen mit dynamischem Charakter der Rahmenbedingungen werden lediglich im Rahmen von Hochrechnungsszenarien vorgenommen.

Mit Blick auf die Ergebnisse der einzelnen Kostenbestandteile hat sich die anfängliche Vermutung bestätigt, dass die Diagnose der Adipositas selbst als Kostendeterminante am wenigsten ins Gewicht fallen würde. Dies hat sich, unter dem Bewusstsein eines wohl unterrepräsentierten Kostenbereichs, mit einem Beitrag von 85,71 Mio. $€$ und einem Anteil von gerade einmal $0,66 \%$ (bzw. $0,67 \%$ bei $6 \%$-igem Diskontierungssatz) an den Gesamtkosten eindrücklich bestätigt. Hier wird es interessant sein, die Wachstumsraten der nächsten Jahre und damit die „Aufwertung“ von Adipositas zu verfolgen. Die 11,265

${ }^{171}$ Schneider, H./ Schmidt, A. (2004). 
Mrd. $€(86,61 \%$ bzw. 88,32\% der Gesamtkosten) an direkten Kosten für Folge- und Begleiterkrankungen stellen im Gegensatz dazu den Hauptanteil der ökonomischen Belastungen dar.

Ein nicht zu verschweigendes Problem in diesem Zusammenhang verbindet sich mit der hohen Sensibilität dieser Kosten in Abhängigkeit der gewählten beizumessenden Anteile. Durch den hohen relativen Anteil dieses Kostenblocks würden bereits kleinere \%Abweichungen der beizumessenden Anteile größere Veränderungen der Kosten und Relationen bedeuten. Umso mehr kommt es darauf an, glaubwürdige und allgemein anerkannte Bezugsgrößen einzusetzen. Insgesamt ist eine Einflussnahme der Adipositas auf andere Erkrankungen als unzweifelhaft anzusehen, allenfalls kann das jeweilige Ausmaß zu Diskussionen führen. Rechnerisch hängen mögliche Kostenverschiebungen zwischen den assoziierten Erkrankungen davon ab, welche Erkrankungen einbezogen und mit welchen epidemiologisch belegbaren relativen Risiken diese schließlich bewertet werden.

Die indirekten Kosten in Höhe von 1,656 Mrd. € (12,68\% der Gesamtkosten) bzw. 1,404 (11,01\% der Gesamtkosten) als Gegenwert für ausgefallene Erwerbsjahre ermöglichen eine weitere Sicht auf die wirtschaftlichen Konsequenzen von Adipositas. Die Schwächen des zugrunde gelegten Ansatzes selbst wurden oben bereits identifiziert und benannt. Stärken liegen einerseits in der Möglichkeit, jene Erkrankungen zu reflektieren, die in Bezug auf die Kriterien Arbeitsunfähigkeit, Invalidität und Mortalität die jeweils bedeutendsten Rollen spielen und zugleich darin, die damit verbundenen, quantitativen Verluste an Humanressourcen zu quantifizieren. Andererseits bewertet das Vorgehen Konsequenzen, ausgehend von einem durch die Adipositas bedingten Ereignisses in der Gegenwart, für die gesamte potenzielle Produktivzeit eines Menschen. Durch die Höhe der Kosten wird die Qualität der Humanressourcen widergespiegelt und je höher diese Qualität - also je geringer die indirekten Kosten - desto eher wird die Bevölkerung in der Lage dazu gesehen, Anpassungen an zukünftige Anforderungen des Gesundheitsbereichs zu bestehen. ${ }^{172}$ Für eine diesbezügliche Einschätzung wären umfangreiche Zeitreihenvergleiche aus der Vergangenheit hilfreich. Für die Bundesrepublik Deutschland erfüllt

\footnotetext{
${ }^{172}$ Henke, K.-D. et al. (1997).
} 
lediglich die Studie des Bundesministeriums für Gesundheit aus dem Jahr 1993 über „Ernährungsabhängige Krankheiten und ihre Kosten“ diesen Anspruch.

\subsection{Einordnung der Ergebnisse und Vergleichsmöglichkeiten}

Um die ermittelten Kosten bzgl. Umfang und Relation einschätzen zu können, wurden Bezugsgrößen wie die gesamten Gesundheitsausgaben (für indirekte Kosten nicht möglich) oder das BIP der Bundesrepublik Deutschland herangezogen. Die Anteile von 0,61\% bzw. 0,60\% am BIP repräsentieren dabei einen statischen volkswirtschaftlichen Verlust für einen definierten Zeitraum. Die wenigen Erhebungen, die zum Vergleich herangezogen werden konnten, sind uneinheitlich und weisen als distributive Top-downAnsätze die vermutete Tendenz auf, in den meisten Fällen zu geringe Kosten auszuweisen.

Wenn sich darüber hinausgehende Vergleiche anboten, dann am ehesten mit Kostenanalysen anderer Problembereiche des Gesundheitswesens, wie z.B. dem Rauchen. In den internationalen Vergleichen mit den USA und Großbritannien und zu Teilen auch mit der Schweiz hat sich gezeigt, dass auch dort die Anteile an adipösen Personen bereits kritische Ausmaße angenommen haben. Während die Kostenbelastung in den USA - wie erwartet - die hohen Prävalenzzahlen weitgehend bestätigte, konnte dieser Nachweis für Großbritannien nicht erbracht und somit die in den Vergleich gesetzten Erwartungen nicht erfüllt werden. Diese Erkenntnis wurde weniger auf ein tatsächlich geringeres Kostenniveau zurückgeführt, als vielmehr auf eine noch unzureichende veröffentlichte Datenlage des Landes, was in weiterführenden Untersuchungen genauer zu analysieren wäre.

\subsection{Hochrechnungen und Zukunftsperspektiven}

Schließlich wurden, ausgehend von den Gesamtkosten des Bezugsjahres, zwei unterschiedliche Hochrechnungsszenarien vorgenommen. Erst dadurch wurde es möglich, ausgehend von den ermittelten statischen Ergebnissen, einen für die weitere Beurteilung wichtigen dynamischen Aspekt einzubringen. Mit den durch die WHO veröffentlichten und zukünftig erwarteten Prävalenzen konnte auf objektive Bezugsgrößen zur wahrscheinlichen Entwicklung der Adipositas in den nächsten Jahren zugegriffen werden. Die Problematiken bei diesen Berechnungen liegen v.a. in der absoluten Unsicherheit der 
Zukunftsereignisse. So basieren die Hochrechnungen zum einen auf WHO-Daten und zum anderen auf ökonomischen Szenarien, die unter bestimmten - wiederum unsicheren - Kriterien aufgestellt wurden. Insofern ist eine Berechnung von Zukunftsszenarien zugleich ein offensiver wie angreifbarer Aspekt. Hinzu kommt die Problematik, dass die Hochrechnungen von einem Bezugsjahr ausgehen, das bereits weiter in der Vergangenheit zurückliegt (2003). In der Konsequenz liegt ein Teil der darauf basierenden ersten Hochrechnungen (2005) ebenfalls bereits in der Vergangenheit. Eine abgleichender IstBerechnungsversuch kann jedoch erst mit einiger Latenzzeit durchgeführt werden, da die Datenverfügbarkeit wie vielfach angesprochen nur spärlich und sehr zeitverzögert gegeben ist, so dass dieser Umstand kaum zu umgehen ist.

Die Kopplung der Szenarien an ein erwartetes durchschnittliches BIP-Wachstum in Höhe von $1,2 \%$ (Szenario 1) bzw. 1,8\% (Szenario 2) sollte ebenfalls zur Objektivität und zur Minimierung spekulativer Aspekte beitragen. Szenario 1 ist von Annahmen ausgegangen, die als gemäßigt einzustufen sind. Dennoch wäre bereits die darin aufgezeigte beinahe Verdopplung der Kostenentwicklung von Adipositas bis ins Jahr 2020 bedenklich und würde mittelfristig das Gesundheitssystem über die Maßen belasten. Um eine möglicherweise eintretende Verdreifachung der Kosten, wie in Szenario 2 denkbar, zu verhindern, wäre es als unumgänglich und dringend erforderlich anzusehen, unverzüglich einschneidende und nachhaltige Veränderungen im Sinne umfassender Präventionsstrategien zu etablieren, sollen die Entwicklungen und Folgen von Adipositas im Rahmen des bestehenden Gesundheitsgefüges in Deutschland unter derartigen Bedingungen zukünftig beherrschbar und finanzierbar bleiben. Auch wenn es sich hierbei um mit Unsicherheiten belegte prognostische Werte handelt, die bei restriktiverer Wahl der zugrunde gelegten Parameter durchaus mildere Konturen annehmen könnten, so sprechen die Anzeichen gegenwärtig doch unmissverständlich für eine weitere Ausbreitung der AdipositasEpidemie und somit auch für weitere drastische Kostenbelastungen.

Neben allen genannten Präventivmassnahmen zur Vermeidung expansiver Adipositasentwicklungen steht v.a. mit der Nationalen Verzehrsstudie II eine viel versprechende Initiative im Blickpunkt, die explizit zwar keine Kostenentwicklung aber dafür repräsentative 
Daten zum Verzehr an Lebensmitteln in der Bevölkerung Deutschlands aufzeigt. ${ }^{173}$ Mit ihr können v.a. längerfristig umfassende Informationen darüber gewonnen werden, welche Nährstoffe die Bundesbürgen zu sich nehmen, welche soziodemographischen Daten dazu in Relation stehen, in welchem Gesundheitszustand sich die Menschen befinden und welchen sie zukünftig mit großer Wahrscheinlichkeit aufweisen werden. Aus diesen Erkenntnissen lassen sich dann konkrete Ernährungsempfehlungen aber auch Prognosen für zukünftige Entwicklungen ableiten. Durch die Initiative hat sich beispielsweise herausgestellt, dass sich nur $4 \%$ der Teilnehmer nach einer definierten Ernährungsweise richten und gerade einmal 8\% den persönlichen Energiebedarf richtig einschätzen können. Auch zeigte sich, dass regional keine Unterschiede bzgl. der Verteilung von Normal-, Übergewicht oder Adipositas bestehen und mit höherem Bildungsstand bei beiden Geschlechtern ein im Durchschnitt geringerer BMI nachzuweisen ist. Zahlreiche weitere Aspekte, wie Koch- und Einkaufsverhalten, Ernährungswissen oder Risikowahrnehmung der Bevölkerung versprechen für die Zukunft wertvolle Ansatzpunkte für Handlungs- und Präventivmaßnahmen.

Auf dieser und den weiteren Initiativen beruhen die Hoffnungen, dass ein gezielt positiver Einfluss auf die Entwicklung der Adipositas genommen werden kann und sich diesbezüglich zu investierende Finanzmittel rechtfertigen und zweckgebundener einsetzen lassen. Allerdings werden die Maßnahmen an dem zukünftigen Erfolg ihrer Nachhaltigkeit und Effektivität gemessen werden, den sie bis dato noch nicht in befriedigendem Maße erbringen konnten.

\footnotetext{
${ }^{173}$ Nationale Verzehrsstudie II des Bundesministeriums für Ernährung, Landwirtschaft und Verbraucherschutz (BMELV), Karlsruhe 2003.
} 


\section{Zusammenfassung}

Die vorliegende Arbeit repräsentiert eine Krankheitskostenanalyse und beschreibt über die Ermittlung von Gesamtkosten die gesundheitsökonomische Belastung, welche durch Adipositas und den zu Adipositas assoziierten Erkrankungen in der Bundesrepublik Deutschland innerhalb eines Bezugsjahres entstanden ist. Für Adipositas und jene 11 als wesentlich identifizierten assoziierten Krankheiten wurden bei einem Diskontierungssatz von 4\%, Kosten in Höhe von 13,007Mrd. $€$ und bei einem Diskontierungssatz von $6 \%$ in Höhe von 12,754 Mrd. $€$ ermittelt. ${ }^{174}$ 86,87\% (bzw. 88,98\%) der Gesamtkosten entfielen auf die direkten und 12,68\% (bzw. 11,01\%) auf die indirekten Kosten. Mit einem Anteil von 56,65\% stellten die in Deutschland zugelassenen Adipositas-Medikamente die größte Kostendeterminante der direkten Kosten für Adipositas, im Sinne der Einzeldiagnosen nach den ICD 10-Ziffern E 65-68, dar. Bei Berücksichtigung aller der Adipositas zuzusprechenden direkten Kosten, dominieren mit großem Abstand die Folgekosten für Diabetes mellitus mit über 6,814 Mrd. €, gefolgt von den Kosten für Herz- und Gefäßerkrankungen in Höhe von 1,920 Mrd. $€$ und Arthrose mit 0,965 Mrd. €. Bei den indirekten Kosten weist die Invalidität mit $46 \%$ oder 760.74 Mio. $€$ (bzw. mit $42 \%$ und 587.31 Mio. $€$ ) den größten relativen Anteil auf. Arbeitsunfähigkeit folgt mit 35\% (41\%). Mortalität repräsentiert mit 19\% (17\%) und den geringsten Kostenanteil. Die indirekten Kosten beinhalten, dass Adipositas in 2003 zu 48.960 Invaliditätsfällen oder 477.938 verlorenen Erwerbsjahren führte. 16.216 verlorene Erwerbsjahre wurden im Zusammenhang mit Arbeitsunfähigkeit und 213.340 aufgrund von frühzeitigen Todesfällen ermittelt.

Mit der Durchführung von Kostenberechnungen im Zusammenhang mit Adipositas in Deutschland verbinden sich eine Reihe von Schwierigkeiten, die zum einen in der teils unzureichenden Datenlage, zum anderen in den zu treffenden methodischen Annahmen zu sehen sind. Im Falle der direkten Kosten steht die restriktive Datensituation im Vordergrund, die bei der Ermittlung der indirekten Kosten keine wesentliche Rolle spielt. Letztere beinhalten dagegen Vorbehalte hinsichtlich ihrer 
Berechnung auf Basis des Humankapital-Ansatzes, u.a. aufgrund der Beschränkung auf die erwerbstätige Bevölkerung im Altersintervall von 15 bis 65 Jahren. Zur Erfassung des epidemiologischen Ausmaßes von Adipositas und den damit verbundenen assoziierten Krankheiten, muss generell auf bevölkerungsspezifische Erhebungen und epidemiologische Studien mit Schwerpunkt auf der Kostenbestimmung zurückgegriffen werden. Aufgrund der nur zu bestimmten Erkrankungen vorhandenen Datenquellen für Deutschland mussten in bestimmten Fällen internationale Analysen herangezogen werden, deren Aussagekraft für Deutschland durchaus eingeschränkt sein kann. Dies ist z.B. an methodischen Abweichungen bzw. an Unterschieden in den Studienvoraussetzungen festzumachen. Insgesamt wurde ein eher konservativer Berechnungsansatz gewählt und die - durch weitestgehend bewiesene Zusammenhänge - wichtigsten zu Adipositas assoziierten Erkrankungen mit in die Untersuchung einbezogen. Internationale Kostenvergleiche zu den USA und Großbritannien wurden durchgeführt. Insbesondere für Großbritannien waren, entgegen den anfänglichen Erwartungen, die ermittelten Ergebnisse nur wenig zufrieden stellend, was in erster Linie mit der unzureichenden Datenlage in Verbindung zu bringen war. Des Weiteren wurden in Hochrechnungen unterschiedliche Szenarien dargelegt, die zugleich eine dynamische wie gesundheitsökonomisch bedenkliche Sichtweise möglicher Kostenentwicklungen von Adipositas aufzeigten.

Trotz der erkannten methodischen und anderer darüber hinausgehender Bedenken liefert diese Krankheitskostenanalyse umfassende Ergebnisse und stellt vor dem Hintergrund der aktuellen Kostendiskussionen im Gesundheitswesen eine Grundlage für gesundheitspolitische bzw. präventive Weichenstellungen zur Bekämpfung der Adipositas in Deutschland dar. Es ist zu hoffen, dass die Ergebnisse aufgegriffen und in weiteren daran anknüpfenden Studien systematische und zyklische Berechnungen für zukünftige Jahre vorgenommen werden.

\footnotetext{
${ }^{174}$ Im weiteren Verlauf werden die Werte bei einem Diskontierungssatz von 6\% in Klammern angegeben.
} 


\section{Literaturverzeichnis}

Allender S., Rayner M. (2006). The burden of obesity-related ill health in the UK. In: Obesity Reviews, Vol 8, Issue 5. S. 467-473.

Assmann, G. (1993): Lipid Metabolism Disorders and Coronary Heart Disease. Münster Heart Study (PROCAM). MMV Medizin Verlag, 1993. S.19-68.

Assmann, G., Schulte, H., Cullen, P. (1997): New and classical risk factors - The Münster Heart Study (PROCAM). Eur J Med Res, 2. S. 237-242.

Bender, R., Jöckel, K.-H., Trautner, C., Spraul, M., Berger, M. (1999): Effect of Age on Excess Mortality in Obesity JAMA, 281, S. 1498-1504.

Bender, R. Jöckel, K.-H., Trautner, C., Spraul, M., Berger, M. (2002): Body Weight, Blood Pressure, and Mortality in a Cohort of Obese Patients. In: Am J Epidemiol 2002; 156. S. 239-245.

Bender, R. (2006): Causes of death in obesity. Relevant increase in cardiovascular but not in all-cancer mortality. In: Journal of Clinical Epidemiology 2006, Volume 59, Issue 10. S. 1064-1071

Benecke, A., Vogel, H. (2005): Übergewicht und Adipositas. In: Robert KochInstitut (Hrsg.), Gesundheitsberichterstattung des Bundes Heft 16.

Birmingham, C. L., Muller, J. L., Palepu, A., Spinelli, J. J., Anis, A. H. (1999): The costs of obesity in Canada. In: CMAJ Canadian Medical Association Journal 2003, 160. S. 483-488.

Bundesministerium für Ernährung, Landwirtschaft und Verbraucherschutz BMELV (2003): Nationale Verzehrsstudie II. Die bundesweite Erhebung zur Ernährung von Jugendlichen und Erwachsenen. Bundesforschungsanstalt für Ernährung und Lebensmittel, Karlsruhe.

Calle, E. E., Rodriguez, C., Walker-Thurmond, K., Thun, M. J. (2003): Overweight, Obesity, and Mortality from Cancer in a Prospectively Studied Cohort of U.S.

Adults. In: The New England Journal of Medicine 2003, Vol. 348. S. 1625-1638.

Colditz, G.A., Willett, W. C., Stampfer, M. J., Manson, J. E., Hennekens, C. H., Arky, R. .A., Speizer, F. E. (1990): Weight as a risk factor for clinical diabetes in women. In: Am J Epidemiol 1990;132. S. 501-513. 
Czerwinski-Mast, M./ Danielzik, S., Asbeck, I., Langnäse, K., Spethmann, C., Müller, M. J. (2003): Kieler Adipositaspräventionsstudie (KOPS) - Konzept und erste Ergebnisse der Vierjahres-Nachuntersuchungen. In:

Bundesgesundheitsblatt - Gesundheitsforschung - Gesundheitsschutz 2003, 46. S. 727-731.

Dreinhöfer, K. E. (2002): Die Bone and Joint Decade - Chancen für Orthopädie und Unfallchirurgie. In: Z Orthop Unfall 2007; 145. S. 399-402.

Fischer, J./ Raschke, F. (1997): Economic and medical significance of sleeprelated breathing disorders. In: Respiration. 1997. 64 Suppl 1. S. 39-44.

Friemel, S., Bernert, S., Angermeyer, M. C., König, H.-H. (2002): Die direkten Kosten von depressiven Erkrankungen in Deutschland. Ergebnisse aus dem European Study of the Epidemiology of Mental Disorders (ESEMeD) Projekt, In: Psychiatrische Praxis. 2005. 32. S. 113-121.

Greiner, W. (Hrsg.) (1999): Ökonomische Evaluation von Gesundheitsleistungen Fragestellungen, Methoden und Grenzen dargestellt am Beispiel der Transplantationsmedizin, In: Gesundheitsökonomische Beiträge, Band 31. Nomos, Baden-Baden.

Hauner, H., Wechsler, J. G., Kluthe, H. , Liebermeister, H., Erbersdobler, H. , Wolfram, G., Fürst, P. , Jauch, K. W. (2000). Qualitätskriterien für ambulante Adipositasprogramme. Eine gemeinsame Initiative der Deutschen AdipositasGesellschaft, Deutschen Akademie für Ernährungsmedizin, Deutschen Gesellschaft für Ernährung, Deutschen Gesellschaft für Ernährungsmedizin. Adipositas, 10, Heft 19. S. 5-8.

Hauner, H., Buchholz, G. , Hamann, A., Husemann, B., Koletzko, B., Liebermeister, H., Wabitsch, M., Westenhöfer, J., Wirth, A., Wolfram, G. (2007) Evidenzbasierte Leitlinie - Prävention und Therapie der Adipositas Version 2007. Deutsche Adipositas-Gesellschaft, Deutsche Diabetes-Gesellschaft und weitere.

Hebebrand, J., Dabrock, P., Lingenfelder, M., Mand, E., Rief, W., Voit, W. (2004): Ist Adipositas eine Krankheit? Interdisziplinäre Perspektiven. In: Deutsches Ärzteblatt 2004, 101 (Heft 37). S. A 2468-2474.

Henke, K.-D., Behrens, C., Arab, L., Schlierf, G. (1986): Die Kosten ernährungsbedingter Krankheiten. In: Schriftenreihe des Bundesministers für Jugend, Familie und Gesundheit, Band 179. Kohlhammer, Stuttgart. 
Henke, K.-D., Martin, K., Behrens, C. (1997): Direkte und indirekte Kosten von Krankheiten in der Bundesrepublik Deutschland 1980 und 1990. In: Wissenschaftliche Dokumentation Fachbereich 14 (Hrsg.): Diskussionspapier 1997/02.

Hubert, H. B., Feinleib, M., McNamara, P. M., Castelli, W. P. (1983): Obesity as an independent risk factor for cardiovascular disease: a 26-year follow-up of participants in the Framingham Heart Study. In: Circulation, Vol 67. S. 968-977. American Heart Association.

Huth, K., Pudel V. (Hrsg.) (1997): Sozioökonomische Bedeutung der Adipositas: Interdisziplinäres Forum „Rhein/ Main-Convent”.

IMS Health (2007): DPM - Der pharmazeutische Markt Deutschland. Statistik über Human-Arzneimittel-Einkäufe öffentlicher Apotheken. Frankfurt am Main.

International Obesity Task Force (IOTF) (1998). Obesity: Preventing and managing the global epidemic. Report of a WHO Consultation on Obesity. Geneva, 3-5 June, 1997. WHO/NUT/NCS/98.1.

Kalarchian, M. A., Marcus, M. D., Levine, M. D., Courcoulas, A. P., Pilkonis, P. A., Ringham, R. M. M, Soulakova, Weissfeld, L. A., Rofey, D. L. (2007): Psychiatric Disorders Among Bariatric Surgery Candidates: Relationship to Obesity and Functional Health Status. In: Am J Psychiatry 164. S. 328-334.

Khaw K. T., Wareham, N., Bingham, S., Welch, A., Luben, R., Day N. (2008): Combined Impact of Health Behaviours and Mortality in Men and Women: The EPIC-Norfolk Prospective Population Study. In: PLoS Medicine, January 2008, Volume 5, Issue 1. S. 39-47.

Köster I., Ferber, L., Ihle, P., Schubert, I., Hauner, H. (2006): The cost burden of diabetes mellitus: the evidence frome Germany - the CoDiM Study. In: Diabetologia 2006, 49. S. 1498-1504.

Kurth, B. M./ Schaffrath Rosario, A. (2007): Die Verbreitung von Übergewicht und Adipositas bei Kindern und Jugendlichen in Deutschland. In: Bundesgesundheitsblatt - Gesundheitsforschung - Gesundheitsschutz, Volume 50, Mai 2007. S. 736-743.

Kohlmeier, L., Kroke, A., Pötzsch, J., Kohlmeier, M., Martin, K. (1993): Ernährungsabhängige Krankheiten und Ihre Kosten. Schriftenreihe des Bundesministeriums für Gesundheit, Band 27. Nomos, Baden-Baden. 
Kolominsky-Rabas, P. L., Heuschmann, P.U., Marschall, D., Emmert, M., Baltzer, N., Neundörfer, B. (2006) Lifetime Cost of Ischemic Stroke in Germany: Results and National Projections From a Population-Based Stroke Registry. The Erlangen Stroke Project. In: Stroke. 2006; 37. S. 1179-1183.

Kossmann, B., Aidelsburger, P., Wasem, J. (2006): Adipositas - eine Krankheit? Explizite und implizite Kriterien für oder gegen die Definition von Adipositas als Krankheit auf Basis medizinischer Leitlinien. Synopse nationaler und internationaler Leitlinien zur Diagnostik und Therapie der Adipositas. Stiftungslehrstuhl für Medizinmanagement, Universität Essen.

Leal, J., Luengo-Fernández, R., Gray, A., Petersen, S., Rayner, M. (2006): Economic burden of cardiavascular diseases in the enlarged European Union. In: European Heart Journal (2006) 27. S. 1610-1619.

Malsch, A. K., Pinheiro, P., Krämer, A., Hornberg, C. (2006): Zur Bestimmung von "Environmental / Burden of Disease“ (BoD / EBD) in Deutschland. In: Landesinstitut für den Öffentlichen Gesundheitsdienst NRW (Hrsg.). Materialien „Umwelt und Gesundheit“ Nr. 65 NRW, Bielefeld, 12/ 2006.

Mc Cusker, M. E. (2004): Disease-Specific Health Care Costs Attributable to Overweight and Obesity - United States, 2004. Paper der California Center for Public Health Advocacy, Davis, CA et al. 2004.

Medizinischer Dienst der Spitzenverbände der Krankenkassen e.V. (MDS): Präventionsbericht 2007 Leistungen der Gesetzlichen Krankenversicherung in der Primärprävention und Betrieblichen Gesundheitsförderung - Berichtsjahr 2006. Asmuth, Köln 2008.

Oberender, P./ Zerth, J. (2006): Adipositas aus gesundheitsökonomischer Sicht: Herausforderung für das Gesundheitssystem. In: Pharmazie in unserer Zeit, Volume 35, Heft 6/ 2006. S. 536-541.

o.V. (2003): Krankheitsartenstatistik 2003. Arbeitsunfähigkeits- und Krankenhausfälle nach Krankheitsarten, Alter, Dauer. In: AOK-Bundesverband (Hrsg.), Bonn 2004.

Paffrath, D / Schwabe, U. (Hrsg.) (2007): AVR 2006 - Aktuelle Daten, Kosten, Trends und Kommentare. Springer, Berlin/ Heidelberg.

Perlitz, U. (2002): Pharmamarkt: Run auf Lifestyle-Drugs von Demografie verstärkt. Sonderausgabe. Deutsche Bank Research (2002) 12. November 2002. Nr. 244. 
Rockhill B, Newman B, Weinberg C. (1988): Use and misuse of population attributable fractions. Am J Public Health 1998; 88. S. 15-19.

Ruff, L. (2000): Gesundheitsökonomische Kosten des Rauchens in Deutschland 1996. Dissertation in Fachbereich Medizin der Universität Hamburg. Medizinische Kernklinik und Poliklinik Universitäts-Krankenhaus Eppendorf, Hamburg.

Schlander, M., Schwarz, O., Thielscher, C. (2004): Gesundheitsausgaben in Deutschland: Eine makroökonomische Analyse ihrer langfristigen

Finanzierbarkeit. In: Kremin-Buch, B. et al. (Hrsg.): Gesundheitsökonomie - Eine Langfristorientierung. S. 83-129. Wissenschaft \& Praxis Dr. Brunner, Sterenfels.

Schneider, H./ Schmidt, A. (2004): Die Kosten der Adipositas in der Schweiz, Schlussbericht für Bundesamt für Gesundheit (BAG), Bern, Schweiz.

Stampfer M. J., Hu F. B., Manson J. E., Rimm, E.B., Willett, W.C. (2000): Primary prevention of coronary heart disease in women through diet and lifestyle. In: $\mathrm{N}$ Engl J Med 343. S. 16-22.

Statistisches Bundesamt (2007/ 2008) (Hrsg.): Statistisches Jahrbuch (mehrere Jahre) - Für die Bundesrepublik Deutschland, Wiesbaden.

Stroh, C., Flade-Kuthe, R., Herbig, B., Höhne, S., Köhler, H., Pick, P., Horbach, T., Weiner, R., Wolff, S., Wolf, A., Schmidt, U., Manger, T. (2007): Studie zur Qualitätskontrolle der operativen Therapie der Adipositas - Ergebnisse der Pilotphase 2005. In: Chir Gastroenterol 2007;23 (suppl 1). S. 55-58.

von Lengerke, T., Reitmeir, P., John J. (2006). Direkte medizinische Kosten der (starken) Adipositas: ein Bottom-up-Vergleich über- vs. normalgewichtiger Erwachsener in der KORA-Studienregion. In: Gesundheitswesen 2006; 68. S. 110-115.

Wang, Y., Lobstein, T. (2006): Worldwide trends in childhood overweight and obesity. In: International Journal of Pediatric Obesity, 2006, 1. S. 11-25.

Weiner, R. A. (2007): Die Zukunft der Adipositas-Chirurgie in Deutschland. In: Chir Gastroenterol 2007, 23 (suppl 1). S.1-3.

Winckler, K. (2005): Organisation der Adipositas- und Ernährungstherapie. In: Actuel Ernaehr Med 2005; 30. S. 30-42.

Wirth, A., Hamann, A., Westenhöfer, J. (2006): Deutsche Adipositas-Gesellschaft: Stellungnahme im Rahmen der Änderung der Anlage 8 der AMR über den Ausschluss von Lifestyle-Arzneimitteln nach § 34, Absatz 1, Satz 7 nF SGB V. 
Wolf, A.M./ Colditz, G.A. (1994): The cost of obesity. In: Pharmaco Economics 1994/ 5 (Suppl 1). S. 34-37.

Wolf A. M./ Manson J. E., Colditz G. A. (2002): The Economic Impact of Overweight, Obesity and Weight Loss. In: Eckel R, (Hrsg.): Obesity: Mechanisms and Clinical Management. Lippincott, Williams and Wilkins.

Wolf, A. M. (2007): Health Economics of Obesity: New Insights. Department of Public Health Sciences University of Virginia School of Medicine (unveröffentlichtes internes Papier).

World Health Association (2003) - Joint WHO/FAO Expert Consultation on Diet, Nutrition and the Prevention of Chronic Diseases. 28 January - 1 February 2002. Technical Report Series 916. WHO, Geneva. Switzerland.

World Health Association (2003) - Der europäische Gesundheitsbericht 2002. In: WHO Regionale Veröffentlichungen (Hrsg.) Europäische Schriftenreihe Nr. 97.

World Health Association Europa (2005). Faktenblatt EURO/13/05: Adipositas: Eine Herausforderung für die Europäische Region der WHO. Kopenhagen, Bukarest. September 2005.

Young, T., Peppard, P. E., Gottlieb, D. J. (2002): Epidemiology of Obstructive Sleep Apnea, A population Health Perspective. In: American Journal of Respiratory and Critical Care Medicine Vol 165 (2002). S. 1217-1239.

Zielke, M., Mark, N. (Hrsg.) (1990) Fortschritte der angewandten Verhaltensmedizin: Konzeption, Grundlagen, Therapie, Evaluation. Springer, Berlin/ Heidelberg.

\section{Online-Publikationen:}

Ampomio - Preisvergleich für Medikamente. Online-Preisvergleichsportal. http://www.apomio.de. Abruf: 16.04.2008.

Apitz, R./ Winter, S. F. (2003): Potenziale und Ansätze der Prävention - aktuelle Entwicklungen in Deutschland. Online-Veröffentlichung unter:

http://www.bmg.bund.de/.../artikelpraevention-pdf-5711.pdf. Abruf: 15.05.2008.

Ärztemagazin Österreich: DFP-Allgemeinmedizin: Akute Pankreatitis. http://www.aerztemagazin.at. Abruf: 02.05.2008. 
BASF AG Finanzbericht 2000: Wertsteigerung durch Wachstum und Innovation. Online-Veröffentlichung unter:

http://www.investor.basf.com/basfcorp/img/investor/finanz d/BASF Fb00.pdf.

Abruf: 14.05.2008.

Bundesärztekammer - Arbeitsgemeinschaft der Deutschen Ärztekammern. http://www.bundesaerztekammer.de/. Abruf: 02.05.2008.

Bundesanstalt für Arbeit uns Soziales: Volkswirtschaftliche Kosten für Arbeitsunfähigkeit. 2003 und 2005. Online-Veröffentlichung unter: http://www.baua.de. Abruf: 14.04.2008.

BMAS - Bundesministerium für Arbeit und Soziales (2005): Lebenslagen in Deutschland. Der 2. Armuts- und Reichtumsbericht der Bundesregierung. OnlineVeröffentlichung unter:

http://www.sozialpolitikaktuell.de/docs/Lebenslagen\%20in\%20Deutschland Bericht.pdf. Abruf: 15.04.2008

Bundesministerium für Gesundheit und soziale Sicherung (2004); Ergebnisse der GKV-Statistik KM 1 (Stand: Januar 2004). http://www.bmg.bund.de. Abruf: 22.05.2008.

Bundesministerium für Gesundheit und soziale Sicherung: gesundheitsziele.de Maßnahmen des Bundesministeriums für Gesundheit zur Umsetzung der nationalen Gesundheitsziele.

http://www.bmg.bund.de. Abruf: 15.05.2008.

Bundesregierung - Referentenentwurf vom 23. November 2007 für ein Gesetz zur Stärkung der Gesundheitsförderung und gesundheitlicher Prävention: OnlineVeröffentlichung unter: http://www.gesundheitberlin.de/download/Referentenentwurf Praeventionsgesetz 11 2007.pdf. Abruf: 14.05.2008.

Deutsche Gesellschaft für Ernährung e.V. www.dge.de/modules.php?name=News\&file=article\&sid=711. Abruf: 25.02.2008.

Deutsche Rentenversicherung Bund (Berlin). Referat 0631 - Statistische Analysen und Methoden.

http://forschung.deutsche-rentenversicherung.de. Abruf am 17.05.2008. 
ERPHO Eastern Region Public Health Obeservatory (United Kingdom) Improving access to population health data, methods and expertise. http://www.erpho.org.uk/topics/Obesity/. Abruf: 05.03.2008.

Eurostat (2003): Health in Europe: results from 1997 to 2000 surveys. European Communities.

http://ec.europa.eu/eurostat Abruf: 12.05.2008.

Gesundheitsberichterstattung des Bundes: Übergewicht und Adipositas Kapitel 2.6.1 [Gesundheit in Deutschland, 2006].

http://www.gbe-bund.de/gbe10. Abruf: 15.01.2008.

Groß, A. F., Günther, K.-P. (2006): Übergewicht und Arthrose: Eine Metaanalyse publizierter Studien. Online-Publikation unter http://www.egms.de/en/meetings/dgu2006/06dgu1005.shtml. Abruf: 10.03.2008.

Health Survey for England (2006): CVD and risk factors adults, obesity and risk factors children. Online-Veröffentlichung unter:

http://www.ic.nhs.uk/pubs/hse06cvdandriskfactors. Abruf: 20.03.2008.

Hessische Krankenhausgesellschaft e.V.: HKG-Report (2005) OnlineVeröffentlichung unter:

http://www.hkg-online.de/info/download/files/HKG 2005 lowENDVERSION.pdf. Abruf: 05.05.2008.

Infomed Verlags AG: Online-Publikation „Die pharma-kritik“ (Wil/ Schweiz) http://www.infomed.org/pharma-kritik. Abruf: 02.05.2008.

Müller, M. J., Maier, H. Mann, R. (2007): Nationaler Aktionsplan gegen das Übergewicht. Eine Initiative der Deutschen Adipositas-Gesellschaft e.V. OnlineVeröffentlichung unter: http://www.adipositas-gesellschaft.de/daten/Nationaler-Aktionsplan-DAG.pdf. Abruf: 16.05.2008.

National Statistics (2000) „Diet \& Nutrition Proportion of overweight children up“. Published 2004. Veröffentlicht unter:

http://www.statistics.gov.uk/cci/nugget.asp?id=718. Abruf: 12.03.2008.

OECD Health Data (2007): Statistics and Indicators for 30 Countries. Paris OECD Publishing. CD-ROM und Online-Publikation:

http://www.oecd.org/health/healthdata. Abruf: 15.04.2008.

OECD Health Data (2007): How Does the United Kingdom Compare. http://www.oecd.org/dataoecd/46/4/38980557.pdf. Abruf: 05.03.2008. 
OECD Gesundheitsdaten (2007): Deutschland im Vergleich. http://www.oecd.org/dataoecd/15/1/39001235.pdf. Abruf am 01.06.2008.

Office for National Statistics (ONS) of the United Kingdom, Health Statistics Quaterly Spring 2008, No. 37. http://www.statistics.gov.uk/downloads/theme health/HSQ37.pdf. Abruf: 06.03.2008.

Plamper, E. (2005): Was wirkt in der Prävention des Rauchens? Vortrag im Rahmen der Dritten Deutschen Konferenz für Tabakkontrolle 8. Dezember 2005. Institut für Gesundheitsökonomie und klinische Epidemiologie der Universität zu Köln. Online-Veröffentlichung unter: http://www.tabakkontrolle.de/pdf/Plamper 2005.pdf. Abruf: 16.05.2008.

Reimann, A. (2007): Aktuelle Entwicklungen in der Rehabilitation. Presse-Vortrag 2007. Deutsche Rentenversicherung Bund, Berlin, 12.07.2007. Vortragsfolien veröffentlicht unter: www.deutsche-rentenversicherung-bund.de. Abruf: 12.05.2008.

Robert-Koch-Institut: Der Kinder- und Jugendgesundheitssurvey - KIGGS-Studie. Online-Veröffentlichung unter: http://www.kiggs.de/studie. Abruf: 08.03.2008.

ROCHE Pharma - Hoffmann-La Roche AG (2004: Geschäftsbericht 2004. OnlineVeröffentlichung unter: http://www.roche.com/pages/downloads/investor/pdf/reports/gb04/gb04d.pdf. Abruf: 14.05.2008.

Sachverständigenrat für die Konzertierte Aktion im Gesundheitswesen (2000): Gesundheitsversorgung und Krankenversicherung 2000 - Mehr Ergebnisorientierung, mehr Qualität und mehr Wirtschaftlichkeit (Kurzfassung und Empfehlungen). http://www.svr-gesundheit.de/Gutachten/Gutacht95/kurzf95.pdf. Abruf: 14.05.2008.

Schaff, M./ Handel, U. (2005): Literaturübersicht Adipositasprävention im Kindesalter (Stand: 2005). Verfasst in wissenschaftlicher Kooperation zwischen dem Bayerischen Landesamt für Gesundheit und Lebensmittelsicherheit und dem Institut für Soziale Pädiatrie der LMU München. www.lgl.bayern.de/gesundheit/doc/adipositaspraevention lit 2005 2.pdf. Abruf: 14.05.2008. 
Statistisches Bundesamt (2006): Bevölkerung Deutschlands bis 2050

Übersicht der Ergebnisse der 11. koordinierten Bevölkerungsvorausberechnung Varianten und zusätzliche Modellrechnungen.

http://www.destatis.de/jetspeed/portal/cms/Sites/destatis/Internet/DE/Content/Stati stiken/Bevoelkerung/VorausberechnungBevoelkerung/Content75/Bevoelkerungse ntwicklung2050Deutschland, property=file.pdf. Abruf: ab 11.04.2008 mehrmalig.

Statistisches Bundesamt der Bundesrepublik Deutschland.

https://www-genesis.destatis.de. Abruf: ab 12.01.2008 mehrmalig.

Statistisches Bundesamt der Bundesrepublik Deutschland: Qualitätsbericht: Krankheitskostenrechnung Methodik bzw. Gesundheit in Deutschland 2006: Vorsorge- oder Rehabilitationseinrichtungen. Online-Veröffentlichung unter: www.gbe-bund.de. Abruf: ab 15.01.2008 mehrmalig.

U.S. Department of Health and Human Services - Centers of Disease Control and Prevention - National Center for Health Statistics.

http://www.cdc.gov/nchs/nhis.htm. Abruf: 05.03.2008.

U.S. Department of Health and Human Services -DHHS Publication No. 2007 1232: Health, United States, 2007 - With Chartbook on Trends in the Health of Americans

http://www.cdc.gov/nchs/data/hus/hus07.pdf\#tocappii. Abruf: 03.03.2008.

U.S. Department of Health and Human Services - Centers of Disease Control and Prevention - Chronic Disease Overview.

http://www.cdc.gov/nccdphp/overview.htm Abruf: 24.03.2008.

Welte, R. Neubauer, S., Leidl, R., (2004): Die Kosten des Zigarettenrauchens, Online-Veröffentlichung unter:

http://www.helmholtzmuenchen.de/neu/Wir ueber uns/jahresbericht/2004/031 036 igm akt.pdf Abruf: 02.04.2008.

Wirth, A. (o. J.): Adipositas - Risikofaktoren und Komorbidität. Online-Publikation unter: ifap Service-Institut für Ärzte und Apotheker GmbH.

http://www.ifap.de/bda-manuale/adipositas/risiken. Abruf: ab 22.04.2008 mehrmalig.

\section{Sonstige:}

Interview mit Frau Dr. Anna Maria Wolf, Leitende Oberärztin des Bereichs Adipositas-Chirurgie der Universitätsklinik Ulm, durchgeführt am 21.05.2008. 


\section{Anhang}

Daten zur Berechnung der indirekten Kosten aufgrund von Arbeitsunfähigkeit

AOK-Arbeitsunfähigkeitsfälle und -tage der Pflichtmitglieder ohne Rentner nach Krankheitsarten 2003 (je 10.000 Personen)

\begin{tabular}{|c|c|c|c|c|c|}
\hline & ICD-Nummer & Fälle & Tage & Fälle & Tage \\
\hline & & \multicolumn{2}{|c|}{ je 10.000 Männer } & \multicolumn{2}{|c|}{ je 10.000 Frauen } \\
\hline Hypertonie & | 10-15 & 100 & 1.876 & 102 & 1.720 \\
\hline Diabetes mellitus (v.a. Typ 2) & E 11-14 & 33 & 825 & 17 & 350 \\
\hline Ischämische Herzerkrankungen & I 20-25, I 50 & 115 & 4.533 & 43 & 1.331 \\
\hline Schlaganfall & I 63-64 & 12 & 969 & 7 & 496 \\
\hline CA der weibl. Geschlechtsorgane & C 51-58 & 0 & 0 & 6 & 395 \\
\hline Prostata-CA & C 61 & 6 & 321 & 0 & 0 \\
\hline Gallenblase/ Pankreas & K 80-87 & 36 & 961 & 56 & 1.199 \\
\hline Arthrose & M 15-19 & 111 & 4.283 & 93 & 3.924 \\
\hline Depression & F 32-34 & 93 & 3.243 & 196 & 6.897 \\
\hline Schlafstörungen/ Schlafapnoe & G 47 & 41 & 291 & 19 & 185 \\
\hline Summe & & 556 & 17.525 & 549 & 16.778 \\
\hline
\end{tabular}

Quelle: Krankheitsartenrechnung AOK Jahr 2003 
AOK-Arbeitsunfähigkeitsfälle und -tage der Pflichtmitglieder ohne Rentner nach Krankheitsarten 2003 insgesamt (ungewichtet) Bezugsgrößen (siehe nachfolgende Tabelle Mitgliederstruktur aller GKV-Versicherten): AOK-Versicherte Männer (4.624.231) und Frauen (3.367.395)

\begin{tabular}{|c|c|c|c|c|c|c|c|}
\hline & ICD-Nummer & Fälle & Tage & $\begin{array}{c}\text { Tage pro } \\
\text { Fall }\end{array}$ & Fälle & Tage & $\begin{array}{c}\text { Tage pro } \\
\text { Fall }\end{array}$ \\
\hline & & \multicolumn{3}{|c|}{ Männer } & \multicolumn{3}{|c|}{ Frauen } \\
\hline Hypertonie & I 10-15 & 46.020 & 866.846 & 19 & 34.522 & 579.788 & 17 \\
\hline Diabetes mellitus (v.a. Typ 2) & E 11-14 & 15.283 & 381.145 & 25 & 5.860 & 118.105 & 20 \\
\hline Ischämische Herzerkrankungen & I 20-25, I 50 & 53.102 & 2.094 .038 & 39 & 14.589 & 448.385 & 31 \\
\hline Schlaganfall & I 63-64 & 5.766 & 447.530 & 78 & 2.329 & 166.987 & 72 \\
\hline Colon-CA & C 18 & 2.573 & 74.313 & 29 & 1.965 & 53.239 & 27 \\
\hline CA der weibl. Geschlechtsorgane & C $51-58$ & 0 & 0 & 0 & 1.968 & 133.233 & 68 \\
\hline Prostata-CA & C 61 & 2.915 & 148.196 & 51 & 0 & 0 & 0 \\
\hline Gallenblase/ Pankreas & K $80-87$ & 16.724 & 443.913 & 27 & 18.751 & 403.952 & 22 \\
\hline Arthrose & M 15-19 & 51.453 & 1.978 .843 & 38 & 31.179 & 1.322 .294 & 42 \\
\hline Depression & F $32-34$ & 43.021 & 1.498 .455 & 35 & 66.052 & 2.324 .289 & 35 \\
\hline Schlafstörungen/ Schlafapnoe & G 47 & 18.762 & 134.345 & 7 & 6.336 & 62.345 & 10 \\
\hline Adipositas & E 65-68 & 1.113 & 29.106 & 26 & 1.466 & 41.451 & 28 \\
\hline Summe & & 256.733 & 8.096 .730 & & 185.016 & 5.654 .068 & \\
\hline
\end{tabular}

Quelle: Eigene Berechnung auf Basis der Krankheitsartenrechnung AOK Jahr 2003. 
AOK-Arbeitsunfähigkeitstage der Pflichtmitglieder ohne Rentner nach Krankheitsarten 2003 insgesamt (mit PAF gewichtet)

\begin{tabular}{|c|c|c|c|c|c|c|c|c|c|}
\hline & ICD-Nummer & \begin{tabular}{|c} 
Tage \\
(ungewichtet)
\end{tabular} & $\begin{array}{c}\text { PAF in } \\
\%\end{array}$ & \begin{tabular}{|c|}
$\begin{array}{c}\text { Tage } \\
\text { (gewichtet) }\end{array}$ \\
\end{tabular} & \begin{tabular}{|c|} 
Relative \\
Anteile \\
$\%$
\end{tabular} & \begin{tabular}{|c|}
$\begin{array}{c}\text { Tage } \\
\text { (ungewichtet) }\end{array}$ \\
\end{tabular} & $\begin{array}{c}\text { PAF in } \\
\%\end{array}$ & $\begin{array}{c}\text { Tage } \\
\text { (gewichtet) }\end{array}$ & $\begin{array}{c}\text { Relative } \\
\text { Anteile \% } \\
\end{array}$ \\
\hline & & \multicolumn{4}{|c|}{ Männer } & \multicolumn{4}{|c|}{ Frauen } \\
\hline Diabetes mellitus (v.a. Typ 2) & E 11-14 & 381.145 & 42,5 & 161.987 & $21,64 \%$ & 118.105 & 42,5 & 50.195 & $10,05 \%$ \\
\hline Ischämische Herzerkrankungen & I 20-25, I 50 & 2.094 .038 & 5,5 & 115.172 & $15,39 \%$ & 448.385 & 5,5 & 24.661 & $4,94 \%$ \\
\hline Schlaganfall & I 63-64 & 447.530 & 3,7 & 16.559 & $2,21 \%$ & 166.987 & 3,7 & 6.179 & $1,24 \%$ \\
\hline CA der weibl. Geschlechtsorgane & C $51-58$ & 0 & 10,3 & 0 & $0,00 \%$ & 133.233 & 10,3 & 13.723 & $2,75 \%$ \\
\hline Prostata-CA & C 61 & 148.196 & 1,6 & 2.371 & $0,32 \%$ & 0 & 1,6 & 0 & $0,00 \%$ \\
\hline Gallenblase/ Pankreas & K 80-87 & 443.913 & 9,7 & 43.060 & $5,75 \%$ & 403.952 & 9,7 & 39.183 & $7,85 \%$ \\
\hline Arthrose & M 15-19 & 1.978 .843 & 9,1 & 180.075 & $24,06 \%$ & 1.322 .294 & 9,1 & 120.329 & $24,10 \%$ \\
\hline Depression & F 32-34 & 1.498 .455 & 5,7 & 85.412 & $11,41 \%$ & 2.324.289 & 5,7 & 132.484 & $26,54 \%$ \\
\hline
\end{tabular}

Quelle: Eigene Berechnung auf Basis der Krankheitsartenrechnung AOK Jahr 2003. 
Mitgliederstruktur aller GKV-Pflichtversicherten in 2003 (Dezember) ohne Rentner und abzüglich Arbeitslose

\begin{tabular}{|c|c|c|c|c|c|c|c|c|c|}
\hline & Insgesamt & AOK & BKK & Innungs-KK & $\begin{array}{l}\text { Landwirt- } \\
\text { schafts-KK }\end{array}$ & $\begin{array}{l}\text { Seekranken- } \\
\text { kasse }\end{array}$ & $\begin{array}{l}\text { Bundes- } \\
\text { knappschaft }\end{array}$ & $\begin{array}{l}\text { Ersatzkassen } \\
\text { Arbeiter }\end{array}$ & $\begin{array}{l}\text { Ersatzassen. } \\
\text { Angestellte }\end{array}$ \\
\hline Männer & 15.186 .018 & 5.755 .887 & 3.815 .821 & 1.481 .308 & 207.037 & 18.959 & 168.925 & 434.133 & 3.303 .948 \\
\hline abzüglich Arbeitslose & 2.302.199 & 1.131 .656 & 325.807 & 268.522 & 731 & 3.092 & 22.869 & 55.392 & 494.130 \\
\hline Gesamt Männer & 12.883 .819 & 4.624.231 & 3.490 .014 & 1.212 .786 & 206.306 & 15.867 & 146.056 & 378.741 & 2.809 .818 \\
\hline Gesamt Männer in \% & $100,00 \%$ & $35,89 \%$ & $27,09 \%$ & $9,41 \%$ & $1,60 \%$ & $0,12 \%$ & $1,13 \%$ & $2,94 \%$ & $21,81 \%$ \\
\hline Frauen & 13.909 .074 & 4.079 .452 & 3.248 .330 & 704.652 & 27.028 & 2.733 & 46.161 & 243.483 & 5.557 .235 \\
\hline abzüglich Arbeitslose & 1.726 .040 & 712.057 & 251.285 & 104.842 & 633 & 502 & 8.458 & 28.341 & 619.922 \\
\hline Gesamt Frauen & 12.183.034 & 3.367 .395 & 2.997 .045 & 599.810 & 26.395 & 2.231 & 37.703 & 215.142 & 4.937 .313 \\
\hline Gesamt Frauen in \% & $100,00 \%$ & $27,64 \%$ & $24,60 \%$ & $4,92 \%$ & $0,22 \%$ & $0,02 \%$ & $0,31 \%$ & $1,77 \%$ & $40,53 \%$ \\
\hline
\end{tabular}

Quelle: Eigene Berechnungen, Basis: Bundesministerium für Gesundheit und soziale Sicherung; Ergebnisse der GKV-Statistik KM 1 (Stand Januar 2004)
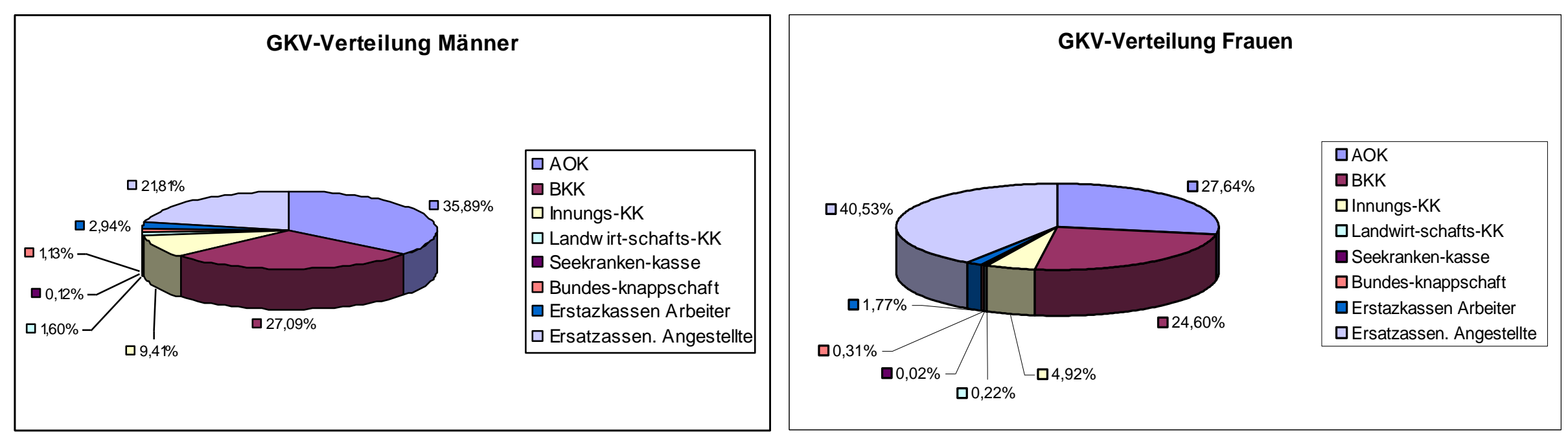
Daten zur Berechnung der indirekten Kosten aufgrund von Invalidität

Fallzahlen an Rentenzugängen im Jahr 2003 aufgrund verminderter Erwerbsfähigkeit/ Invalidität nach Geschlecht und Altersgruppen

\begin{tabular}{|c|c|c|c|c|c|c|c|c|}
\hline männliche Bevölkerung & ICD 10 Ziffern & & Rentenzu & gänge i & $2003 \mathrm{Ge}$ & tzliche R & ntenvers & erung \\
\hline & & bis 39 & $40-44$ & $45-49$ & $50-54$ & $55-59$ & 60 plus & Renten insgesamt \\
\hline Hypertonie & | 10 bis I 15 & 27 & 56 & 129 & 297 & 625 & 284 & 1.418 \\
\hline Diabetes mellitus & E 11 bis E 14 & 35 & 107 & 253 & 652 & 801 & 242 & 2.090 \\
\hline Ischämische Herzkrankheiten & I 20 bis I 25 und I 50 & 78 & 222 & 586 & 1.337 & 2.184 & 949 & 5.356 \\
\hline Schlaganfall & I 63 bis I 64 & 103 & 184 & 390 & 787 & 1.216 & 479 & 3.159 \\
\hline Colon CA & C 18 & 16 & 48 & 70 & 182 & 249 & 96 & 661 \\
\hline CA weibliche Geschlechtsorgane & C 51 bis C 58 & 0 & 0 & 0 & 0 & 0 & 0 & 0 \\
\hline Prostata CA & C 61 & 3 & 5 & 43 & 150 & 442 & 182 & 825 \\
\hline Galle/ Pankreas & $\mathrm{K} 80$ bis $\mathrm{K} 87$ & 49 & 74 & 100 & 78 & 59 & 14 & 374 \\
\hline Arthrose & M 15 bis M 19 & 98 & 189 & 446 & 1.141 & 2.023 & 719 & 4.616 \\
\hline Depression & $\mathrm{F} 32$ bis $\mathrm{F} 34$ & 398 & 464 & 757 & 1.414 & 1.734 & 358 & 5.125 \\
\hline Schlafapnoe & G 47 & 5 & 9 & 14 & 26 & 25 & 11 & 90 \\
\hline Adipositas & E 65 bis E 68 & 16 & 51 & 59 & 81 & 99 & 22 & 328 \\
\hline
\end{tabular}

\begin{tabular}{|c|c|c|c|c|c|c|c|c|}
\hline \multirow[t]{2}{*}{ weibliche Bevölkerung } & \multirow[t]{2}{*}{ ICD 10 Ziffern } & \multicolumn{7}{|c|}{ Rentenzugänge 2003 Gesetzliche Rentenversicherung } \\
\hline & & bis 39 & $40-44$ & $45-49$ & $50-54$ & $55-59$ & 60 plus & Rente insgesamt \\
\hline Hypertonie & I 10 bis I 15 & 18 & 27 & 58 & 170 & 331 & 85 & 689 \\
\hline Diabetes mellitus & E 11 bis E 14 & 20 & 47 & 96 & 208 & 331 & 68 & 770 \\
\hline Ischämische Herzkrankheiten & | 20 bis I 25 und I 50 & 30 & 55 & 105 & 253 & 388 & 91 & 922 \\
\hline Schlaganfall & I 63 bis I 64 & 112 & 125 & 225 & 352 & 392 & 85 & 1.291 \\
\hline Colon CA & C 18 & 23 & 38 & 70 & 139 & 175 & 32 & 477 \\
\hline CA weibliche Geschlechtsorgane & C 51 bis C 58 & 144 & 192 & 284 & 356 & 401 & 66 & 1.443 \\
\hline Prostata CA & C 61 & 0 & 0 & 0 & 0 & 0 & 0 & 0 \\
\hline Galle/ Pankreas & $\mathrm{K} 80$ bis $\mathrm{K} 87$ & 16 & 11 & 31 & 20 & 19 & 3 & 100 \\
\hline Arthrose & M 15 bis M 19 & 101 & 127 & 339 & 946 & 1.528 & 351 & 3.392 \\
\hline Depression & F 32 bis $F 34$ & 734 & 926 & 1.546 & 2.840 & 2.777 & 234 & 9.057 \\
\hline Schlafapnoe & G 47 & 5 & 0 & 6 & 8 & 10 & 1 & 30 \\
\hline Adipositas & E 65 bis E 68 & 19 & 28 & 55 & 89 & 98 & 13 & 302 \\
\hline
\end{tabular}

Quelle: VDR Würzburg, Referat Statistisches Berichtswesen 


\section{Berechnung der verlorenen Erwerbsjahre durch Invalidität}

\section{Annahmen:}

Es existieren keine spezifischen personellen Unterschiede oder Risiken in der Bevölkerung außer Alter und Geschlecht. Jede Person innerhalb einer Altersgruppe weist eine identische Überlebensquote auf.

Gleichverteilung der Invaliditätsfälle innerhalb der jeweiligen Altersgruppe.

Alle Werte in der Zukunftssicht bleiben konstant.

Die Lebenserwartung bleibt unberücksichtigt, da sie durchweg höher liegt als das höchste Erwerbsalter von 65 Jahren.

Für die Altersgruppe "unter 39 Jahren" wird mit einem Intervall "30-39 Jahre" gerechnet (Annahme: unter 30 geringe Invaliditätsrate)

Die Überlebensquote der unter 39-Jährigen wird mit dem Durchschnittwert aller Altersgruppen bis 39 berechnet $(98,5 \%)$

Die Altersgruppe "60 und höher" wird als "60-65 Jahre" betrachtet (reguläres Rentenalter).

Die unterschiedliche Darstellung der Altersgruppenintervalle der herangezogenen Statistiken bleiben unberücksichtigt (grundsätzlich gilt ein 5-

Jahresintervall)

\section{Vorgehen:}

1.) Ermittlung der Invaliditätsfallzahlen $x$ Überlebensquote je Altersgruppe und Geschlecht pro Erkrankung nach ICD 10

2.) Berechnung von verlorenen Erwerbsjahren je Altersgruppe und Geschlecht $=$ (niedrigster Wert je Altersgruppe + höchster Wert je Altersgruppe) $: 2=$

$[\mathrm{X}+(\mathrm{X}+5)]: 2$

3.) Bewertung der verlorenen Erwerbsjahre und Diskontierung (4\% und 6\%)

\section{Berechnungsschritt 1.) Ermittlung der Invaliditätsfallzahlen $x$ Überlebensquote}

\section{Überlebensquote 2003, nach Altersgruppen (Berechnet als Mittelwert aus dem Anteil an Überlebensjahren pro Altersgruppe) Angaben in \%}

\begin{tabular}{|c|c|c|c|c|c|c|}
\hline Altersgruppe & bis 39 & $40-45$ & $45-50$ & $50-55$ & $55-60$ & $60-65$ \\
\hline männliche Bevölkerung & 98,5 & 96,8 & 95,3 & 93,0 & 89,8 & 84,9 \\
\hline weibliche Bevölkerung & 99,1 & 98,2 & 97,5 & 96,3 & 94,5 & 92,0 \\
\hline
\end{tabular}

Quelle: Statistisches Jahrbuch 2006 für die Bundesrepublik Deutschland: Sterbetafeln (2002/2004), Absterbeordnung

Berechnung: (niedrigster Wert Altersgruppe + höchster Wert Altersgruppe) : $2=[X+(X+5)]: 2$ (angegeben in Prozent) 


\section{Berechnungsschritt 1.) Ermittlung der Invaliditätsfallzahlen x Überlebensquote (Fortsetzung)}

\begin{tabular}{|c|c|c|c|c|c|c|c|c|}
\hline männliche Bevölkerung & ICD 10 Ziffern & Rente & ugänge 2 & 3 mit Berì & sichtigun & der Überle & nswahrsch & ichkeit \\
\hline & & bis 39 & $40-44$ & $45-49$ & $50-54$ & $55-59$ & 60 plus & $\Sigma$ Renten \\
\hline Hypertonie & I 10 bis I 15 & 27 & 54 & 123 & 276 & 561 & 241 & 1.282 \\
\hline Diabetes mellitus & $\mathrm{E} 11$ bis $\mathrm{E} 14$ & 34 & 104 & 241 & 606 & 719 & 205 & 1.910 \\
\hline Ischäm. Herzkrankheiten & I 20 bis I 25 und I 50 & 77 & 215 & 558 & 1.243 & 1.961 & 806 & 4.861 \\
\hline Schlaganfall & I 63 bis I 64 & 101 & 178 & 372 & 732 & 1.092 & 407 & 2.882 \\
\hline Colon CA & C 18 & 16 & 46 & 67 & 169 & 224 & 82 & 603 \\
\hline CA $q$ Geschlechtsorgane & C 51 bis C 58 & 0 & 0 & 0 & 0 & 0 & 0 & 0 \\
\hline Prostata CA & C 61 & 3 & 5 & 41 & 140 & 397 & 155 & 740 \\
\hline Galle/ Pankreas & $\mathrm{K} 80$ bis $\mathrm{K} 87$ & 48 & 72 & 95 & 73 & 53 & 12 & 353 \\
\hline Arthrose & M 15 bis M 19 & 97 & 183 & 425 & 1.061 & 1.817 & 610 & 4.193 \\
\hline Depression & $\mathrm{F} 32$ bis $\mathrm{F} 34$ & 392 & 449 & 721 & 1.315 & 1.557 & 304 & 4.739 \\
\hline Schlafapnoe & G 47 & 5 & 9 & 13 & 24 & 22 & 9 & 83 \\
\hline Adipositas & E 65 bis E 68 & 16 & 49 & 56 & 75 & 89 & 19 & 304 \\
\hline
\end{tabular}

\begin{tabular}{|c|c|c|c|c|c|c|c|c|}
\hline \multirow[t]{2}{*}{ weibliche Bevölkerung } & \multirow[t]{2}{*}{ ICD 10 Ziffern } & \multicolumn{7}{|c|}{ Rentenzugänge 2003 mit Berücksichtigung der Überlebenswahrscheinlichkeit } \\
\hline & & bis 39 & $40-44$ & $45-49$ & $50-54$ & $55-59$ & 60 plus & $\Sigma$ Renten \\
\hline Hypertonie & I 10 bis I 15 & 18 & 27 & 57 & 164 & 313 & 78 & 656 \\
\hline Diabetes mellitus & E 11 bis E 14 & 20 & 46 & 94 & 200 & 313 & 63 & 735 \\
\hline Ischäm. Herzkrankheiten & I 20 bis I 25 und I 50 & 30 & 54 & 102 & 244 & 367 & 84 & 880 \\
\hline Schlaganfall & I 63 bis I 64 & 111 & 123 & 219 & 339 & 370 & 78 & 1.241 \\
\hline Colon CA & C 18 & 23 & 37 & 68 & 134 & 165 & 29 & 457 \\
\hline CA $\&$ Geschlechtsorgane & C 51 bis C 58 & 143 & 189 & 277 & 343 & 379 & 61 & 1.391 \\
\hline Prostata CA & C 61 & 0 & 0 & 0 & 0 & 0 & 0 & 0 \\
\hline Galle/ Pankreas & $\mathrm{K} 80$ bis $\mathrm{K} 87$ & 16 & 11 & 30 & 19 & 18 & 3 & 97 \\
\hline Arthrose & M 15 bis M 19 & 100 & 125 & 331 & 911 & 1.444 & 323 & 3.233 \\
\hline Depression & F 32 bis F 34 & 727 & 909 & 1.507 & 2.735 & 2.624 & 215 & 8.719 \\
\hline Schlafapnoe & G 47 & 5 & 0 & 6 & 8 & 9 & 1 & 29 \\
\hline Adipositas & E 65 bis E 68 & 19 & 27 & 54 & 86 & 93 & 12 & 290 \\
\hline
\end{tabular}

Quelle: Eigene Berechnungen auf Basis der angegebenen Datenquellen. 
Berechnungsschritt 2.) Berechnung der verlorenen Erwerbsjahre je Altersgruppe und Geschlecht

(Altersgruppe bis 39 verliert vereinfachend [(65 - 30) + (65 - 39)] : 2 = 30,5 Jahre, alle weiteren Altersgruppen analog zur Berechnung "Mortalität")

\begin{tabular}{|c|c|c|c|c|c|c|c|c|}
\hline männliche Bevölkerung & ICD 10 Ziffern & & Verl & ene Erwer & jahre aufo & Ind von Ir & alidität & \\
\hline & & bis 39 & $40-44$ & $45-49$ & $50-54$ & $55-59$ & 60 plus & Jahre insgesamt \\
\hline Hypertonie & I 10 bis I 15 & 811,1 & $1.219,7$ & $2.151,4$ & $3.452,6$ & $4.209,4$ & 602,8 & $12.447,0$ \\
\hline Diabetes mellitus & E 11 bis E 14 & $1.051,5$ & $2.330,5$ & $4.219,4$ & $7.579,5$ & $5.394,7$ & 513,6 & $21.089,2$ \\
\hline Ischämische Herzkrankheiten & I 20 bis I 25 und I 50 & $2.343,3$ & $4.835,2$ & $9.773,0$ & $15.542,6$ & $14.709,2$ & $2.014,3$ & $49.217,6$ \\
\hline Schlaganfall & I 63 bis I 64 & $3.094,4$ & $4.007,5$ & $6.504,2$ & $9.148,9$ & $8.189,8$ & $1.016,7$ & $31.961,4$ \\
\hline Colon CA & C 18 & 480,7 & $1.045,4$ & $1.167,4$ & $2.115,8$ & $1.677,0$ & 203,8 & $6.690,1$ \\
\hline CA weibliche Geschlechtsorgane & C 51 bis C 58 & 0,0 & 0,0 & 0,0 & 0,0 & 0,0 & 0,0 & 0,0 \\
\hline Prostata CA & C 61 & 90,1 & 108,9 & 717,1 & $1.743,8$ & $2.976,9$ & 386,3 & $6.023,1$ \\
\hline Galle/ Pankreas & $\mathrm{K} 80$ bis $\mathrm{K} 87$ & $1.472,1$ & $1.611,7$ & $1.667,8$ & 906,8 & 397,4 & 29,7 & $6.085,4$ \\
\hline Arthrose & M 15 bis M 19 & $2.944,2$ & $4.116,4$ & $7.438,2$ & $13.264,1$ & $13.624,9$ & $1.526,1$ & $42.913,9$ \\
\hline Depression & F 32 bis F 34 & $11.956,9$ & $10.105,9$ & $12.624,9$ & $16.437,8$ & $11.678,5$ & 759,9 & $63.563,8$ \\
\hline Schlafapnoe & G 47 & 150,2 & 196,0 & 233,5 & 302,3 & 168,4 & 23,3 & $1.073,7$ \\
\hline Adipositas & E 65 bis E 68 & 480,7 & $1.110,8$ & 984,0 & 941,6 & 666,8 & 46,7 & $4.230,5$ \\
\hline Summe & & $24.875,2$ & $30.688,0$ & $47.480,8$ & $71.435,6$ & $63.692,9$ & $7.123,1$ & $245.295,7$ \\
\hline weibliche Bevölkerung & ICD 10 Ziffern & bis 39 & $40-44$ & $45-49$ & $50-54$ & $55-59$ & 60 plus & Jahre insgesamt \\
\hline Hypertonie & | 10 bis I 15 & 544,1 & 596,6 & 989,6 & $2.046,4$ & $2.346,0$ & 195,5 & $6.718,1$ \\
\hline Diabetes mellitus & E 11 bis E 14 & 604,5 & $1.038,5$ & $1.638,0$ & $2.503,8$ & $2.346,0$ & 156,4 & $8.287,1$ \\
\hline Ischämische Herzkrankheiten & I 20 bis I 25 und I 50 & 906,8 & $1.215,2$ & $1.791,6$ & $3.045,5$ & $2.750,0$ & 209,3 & $9.918,3$ \\
\hline Schlaganfall & 163 bis I 64 & $3.385,3$ & $2.761,9$ & $3.839,1$ & $4.237,2$ & $2.778,3$ & 195,5 & $17.197,2$ \\
\hline Colon CA & C 18 & 695,2 & 839,6 & $1.194,4$ & $1.673,2$ & $1.240,3$ & 73,6 & $5.716,3$ \\
\hline CA weibliche Geschlechtsorgane & C 51 bis C 58 & $4.352,5$ & $4.242,2$ & $4.845,8$ & $4.285,4$ & $2.842,1$ & 151,8 & $20.719,7$ \\
\hline Prostata CA & C 61 & 0,0 & 0,0 & 0,0 & 0,0 & 0,0 & 0,0 & 0,0 \\
\hline Galle/ Pankreas & $\mathrm{K} 80$ bis $\mathrm{K} 87$ & 483,6 & 243,0 & 528,9 & 240,8 & 134,7 & 6,9 & $1.637,9$ \\
\hline Arthrose & M 15 bis M 19 & $3.052,8$ & $2.806,1$ & $5.784,2$ & $11.387,5$ & $10.829,7$ & 807,3 & $34.667,5$ \\
\hline Depression & $\mathrm{F} 32$ bis $\mathrm{F} 34$ & $22.185,5$ & $20.460,0$ & $26.378,6$ & $34.186,5$ & $19.682,0$ & 538,2 & $123.430,8$ \\
\hline Schlafapnoe & G 47 & 151,1 & 0,0 & 102,4 & 96,3 & 70,9 & 2,3 & 423,0 \\
\hline Adipositas & E 65 bis E 68 & 574,3 & 618,7 & 938,4 & $1.071,3$ & 694,6 & 29,9 & $3.927,2$ \\
\hline Summe & & $36.935,6$ & $34.821,7$ & $48.030,9$ & $64.773,8$ & $45.714,4$ & $2.366,7$ & $232.643,1$ \\
\hline
\end{tabular}

Quelle: Eigene Berechnungen auf Basis der angegebenen Datenquellen. 
Berechnungsschritt 3.) Monetäre Bewertung der verlorenen Erwerbsjahre je Altersgruppe und Diskontierung mit 4\% und $6 \%$

Mit 4\% Diskontierungssatz und $27.493 €$ Bruttoeinkommen (Voll- und Teilzeit) aus unselbständiger Arbeit (2003)

\begin{tabular}{|c|c|c|c|c|c|c|c|c|}
\hline \multicolumn{2}{|c|}{ männliche Bevölkerung 4\% Diskont in Euro } & bis 39 & $40-44$ & $45-49$ & $50-54$ & $55-59$ & 60 plus & Summe \\
\hline Hypertonie & I 10 bis $\mid 15$ & $6.742 .253,5$ & $13.874 .517,9$ & $29.775 .520,9$ & $58.991 .599,9$ & $86.236 .216,0$ & $15.024 .666,6$ & 210.644.774,7 \\
\hline Diabetes mellitus & E 11 bis E 14 & $8.739 .958,2$ & $26.510 .239,6$ & $58.396 .951,8$ & $129.503 .444,8$ & $110.520 .334,4$ & $12.802 .708,9$ & $346.473 .637,7$ \\
\hline Ischämische Herzkrankheiten & 120 bis 125,150 & 19.477.621,2 & $55.002 .553,2$ & 135.259.342,9 & $265.561 .511,9$ & $301.343 .833,1$ & $50.205 .664,1$ & $826.850 .526,3$ \\
\hline Schlaganfall & I 63 bis I 64 & $25.720 .448,5$ & $45.587 .701,7$ & $90.019 .016,6$ & $156.317 .808,4$ & $167.781 .181,8$ & $25.340 .899,0$ & $510.767 .056,0$ \\
\hline Colon CA & C 18 & $3.995 .409,5$ & $11.892 .443,9$ & $16.157 .259,4$ & $36.149 .734,6$ & $34.356 .508,4$ & $5.078 .760,5$ & $107.630 .116,4$ \\
\hline CA $q$ Geschlechtsorgane & C 51 bis C 58 & & & & & & & \\
\hline Prostata CA & C 61 & $749.139,3$ & $1.238 .796,2$ & $9.925 .173,6$ & $29.793 .737,3$ & $60.986 .251,9$ & $9.628 .483,5$ & 112.321.581,9 \\
\hline Galle/ Pankreas & $\mathrm{K} 80$ bis $\mathrm{K} 87$ & $12.235 .941,5$ & $18.334 .184,4$ & $23.081 .799,1$ & $15.492 .743,4$ & $8.140 .698,8$ & $740.652,6$ & $78.026 .019,8$ \\
\hline Arthrose & M 15 bis M 19 & $24.471 .883,1$ & $46.826 .498,0$ & $102.944 .824,1$ & $226.631 .028,4$ & $279.129 .383,8$ & $38.037 .800,3$ & $718.041 .417,8$ \\
\hline Depression & F 32 bis F 34 & $99.385 .810,9$ & $114.960 .291,3$ & $174.729 .219,4$ & $280.855 .630,3$ & $239.253 .757,6$ & $18.939 .544,5$ & 928.124.254,1 \\
\hline Schlafapnoe & G 47 & $1.248 .565,5$ & 2.229.833,2 & $3.231 .451,9$ & $5.164 .247,8$ & $3.449 .448,6$ & $581.941,3$ & $15.905 .488,3$ \\
\hline Adipositas & $\mathrm{E} 65$ bis $\mathrm{E} 68$ & $3.995 .409,5$ & 12.635.721,7 & $13.618 .261,5$ & $16.088 .618,1$ & $13.659 .816,6$ & $1.163 .882,6$ & $61.161 .710,0$ \\
\hline & & & & & & Sum & & $3.915 .946 .583,1$ \\
\hline
\end{tabular}

\begin{tabular}{|c|c|c|c|c|c|c|c|c|}
\hline \multicolumn{2}{|c|}{ weibliche Bevölkerung 4\% Diskont in Euro } & \multirow{2}{*}{\begin{tabular}{c|} 
bis 39 \\
$4.522 .215,4$
\end{tabular}} & \multirow{2}{*}{\begin{tabular}{|c|}
$40-44$ \\
$6.786 .248,7$ \\
\end{tabular}} & \multirow{2}{*}{$\frac{45-49}{13.696 .492,6}$} & \multirow{2}{*}{$\frac{50-54}{34.964 .392,4}$} & \multirow{2}{*}{\begin{tabular}{c|}
$55-59$ \\
$48.061 .037,3$
\end{tabular}} & \multirow{2}{*}{\begin{tabular}{|c|}
60 plus \\
$4.872 .878,3$
\end{tabular}} & \multirow{2}{*}{$\begin{array}{r}\text { Jahre insgesamt } \\
112.903 .264,6\end{array}$} \\
\hline Hypertonie & I 10 bis I 15 & & & & & & & \\
\hline Diabetes mellitus & E 11 bis E 14 & $5.024 .683,8$ & $11.813 .099,5$ & $22.670 .056,6$ & $42.779 .962,4$ & $48.061 .037,3$ & $3.898 .302,7$ & 134.247.142,3 \\
\hline Ischämische Herzkrankheiten & | 20 bis $\mid 25,150$ & $7.537 .025,6$ & $13.823 .839,9$ & $24.795 .374,5$ & $52.035 .242,7$ & $56.337 .409,3$ & $5.216 .846,2$ & 159.745.738,2 \\
\hline Schlaganfall & I 63 bis I 64 & $28.138 .229,0$ & $31.417 .817,9$ & $53.132 .945,3$ & $72.396 .859,5$ & $56.918 .207,3$ & 4.872.878,3 & 246.876.937,3 \\
\hline Colon CA & C 18 & $5.778 .386,3$ & $9.551 .016,7$ & $16.530 .249,6$ & $28.588 .532,6$ & $25.409 .914,0$ & $1.834 .495,4$ & $87.692 .594,5$ \\
\hline CA $q$ Geschlechtsorgane & C 51 bis C 58 & $36.177 .723,0$ & $48.257 .768,4$ & $67.065 .584,2$ & $73.219 .551,1$ & $58.225 .002,9$ & $3.783 .646,7$ & 286.729.276,2 \\
\hline Prostata CA & C 61 & & & & & & & 0,0 \\
\hline Galle/ Pankreas & $\mathrm{K} 80$ bis $\mathrm{K} 87$ & $4.019 .747,0$ & $2.764 .768,0$ & $7.320 .539,1$ & $4.113 .457,9$ & $2.758 .790,7$ & $171.983,9$ & 21.149.286,6 \\
\hline Arthrose & M 15 bis M 19 & $25.374 .653,0$ & $31.920 .503,0$ & $80.053 .637,5$ & $194.566 .559,8$ & $221.864 .848,8$ & $20.122 .121,1$ & $573.902 .323,2$ \\
\hline Depression & $\mathrm{F} 32$ bis $\mathrm{F} 34$ & $184.405 .893,8$ & $232.743 .195,3$ & $365.082 .370,6$ & $584.111 .025,3$ & $403.219 .034,8$ & $13.414 .747,4$ & 1.782.976.267, 1 \\
\hline Schlafapnoe & G 47 & 1.256.170,9 & 0,0 & $1.416 .878,5$ & $1.645 .383,2$ & $1.451 .995,1$ & $57.328,0$ & $5.827 .755,7$ \\
\hline Adipositas & E 65 bis E 68 & $4.773 .449,6$ & $7.037 .591,2$ & $12.988 .053,3$ & $18.304 .887,8$ & $14.229 .551,8$ & $745.263,7$ & $58.078 .797,4$ \\
\hline & & & & & & Sum & & 3.470.129.383,2 \\
\hline
\end{tabular}


Monetäre Bewertung der verlorenen Erwerbsjahre je Altersgruppe mit 6\% Diskontierungssatz und $27.493 €$ Bruttoeinkommen (Voll- und Teilzeit) aus unselbst. Arbeit (2003)

\begin{tabular}{|c|c|c|c|c|c|c|c|c|}
\hline \multicolumn{2}{|c|}{ männliche Bevölkerung 6\% Diskont in Euro } & \multirow{2}{*}{\begin{tabular}{|c|} 
bis 39 \\
$3.771 .319,4$ \\
\end{tabular}} & \multirow{2}{*}{\begin{tabular}{|c|}
$40-44$ \\
$9.038 .286,5$ \\
\end{tabular}} & \multirow{2}{*}{\begin{tabular}{|c|}
$45-49$ \\
$21.334 .876,7$ \\
\end{tabular}} & \multirow{2}{*}{$\begin{array}{c}50-54 \\
45.819 .274,9\end{array}$} & \multirow{2}{*}{\begin{tabular}{|c|}
$55-59$ \\
$74.755 .952,4$ \\
\end{tabular}} & \multirow{2}{*}{$\begin{array}{c}60 \text { plus } \\
14.325 .953,2\end{array}$} & \multirow{2}{*}{$\begin{array}{c}\text { Summe } \\
169.045 .663,0\end{array}$} \\
\hline Hypertonie & I 10 bis I 15 & & & & & & & \\
\hline Diabetes mellitus & E 11 bis $\mathrm{E} 14$ & $4.888 .747,3$ & $17.269 .583,1$ & $41.842 .820,3$ & $100.586 .421,6$ & $95.807 .228,6$ & 12.207.326,3 & 272.602.127,2 \\
\hline Ischämische Herzkrankheiten & I 20 bis I 25,150 & $10.894 .922,6$ & $35.830 .350,0$ & $96.916 .571,8$ & $206.263 .873,8$ & $261.227 .200,0$ & $47.870 .878,8$ & $659.003 .797,0$ \\
\hline Schlaganfall & I 63 bis I 64 & $14.386 .885,0$ & $29.697 .227,0$ & $64.500 .790,1$ & $121.413 .364,8$ & $145.445 .181,0$ & $24.162 .435,2$ & $399.605 .883,0$ \\
\hline Colon CA & C 18 & $2.234 .855,9$ & $7.747 .102,7$ & $11.577 .064,9$ & $28.077 .804,8$ & 29.782.771,4 & $4.842 .575,7$ & $84.262 .175,5$ \\
\hline CA $q$ Geschlechtsorgane & C 51 bis C 58 & & & & & & & 0,0 \\
\hline Prostata CA & C 61 & $419.035,5$ & $806.989,9$ & $7.111 .625,6$ & 23.141.047,9 & $52.867 .409,5$ & $9.180 .716,5$ & 93.526.824,9 \\
\hline Galle/ Pankreas & $\mathrm{K} 80$ bis $\mathrm{K} 87$ & $6.844 .246,2$ & $11.943 .450,0$ & $16.538 .664,1$ & $12.033 .344,9$ & $7.056 .961,9$ & $706.209,0$ & $55.122 .876,1$ \\
\hline Arthrose & M 15 bis M 19 & $13.688 .492,5$ & $30.504 .216,9$ & $73.762 .442,1$ & 176.026.237,9 & $241.970 .066,7$ & $36.268 .874,5$ & $572.220 .330,4$ \\
\hline Depression & $\mathrm{F} 32$ bis $\mathrm{F} 34$ & $55.592 .040,9$ & $74.888 .659,4$ & 125.197.687,5 & $218.142 .945,1$ & $207.402 .914,3$ & 18.058.772,0 & $699.283 .019,1$ \\
\hline Schlafapnoe & G 47 & $698.392,5$ & $1.452 .581,8$ & $2.315 .413,0$ & $4.011 .115,0$ & $2.990 .238,1$ & $554.878,5$ & 12.022.618,7 \\
\hline Adipositas & E 65 bis E 68 & $2.234 .855,9$ & $8.231 .296,6$ & $9.757 .811,8$ & $12.496 .165,9$ & $11.841 .342,9$ & $1.109 .756,9$ & $45.671 .230,0$ \\
\hline & & & & & & Sum & & $3.062 .366 .545,0$ \\
\hline
\end{tabular}

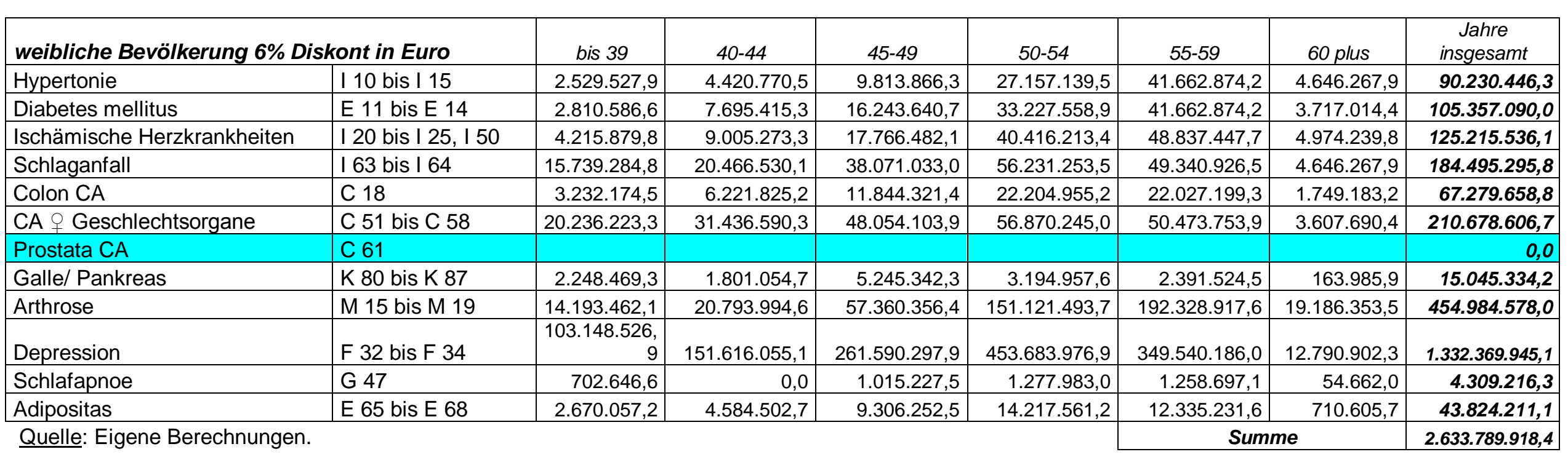


Daten zur Berechnung der indirekten Kosten aufgrund von Mortalität

Sterbefälle je 100.000 Einwohner (2003), männliche Bevölkerung, nach Erkrankung (ICD 10) und Altersgruppen

\begin{tabular}{|c|c|c|c|c|c|c|c|c|c|c|}
\hline \multicolumn{11}{|l|}{ männliche Bevölkerung } \\
\hline Erkrankung/ Altersgruppe & $15-20$ & $20-25$ & $25-30$ & $30-35$ & $35-40$ & $40-45$ & $45-50$ & $50-55$ & $55-60$ & $60-65$ \\
\hline Hypertonie & 0,0 & 0,1 & 0,1 & 0,1 & 1,0 & 1,3 & 3,7 & 6,3 & 9,8 & 16,9 \\
\hline Diabetes mellitus & 0,2 & 0,2 & 0,4 & 0,9 & 1,2 & 2,9 & 5,5 & 10,1 & 15,9 & 28,7 \\
\hline KHK & 1,3 & 1,4 & 2,9 & 4,2 & 10,6 & 26,0 & 54,7 & 94,9 & 155,9 & 266,0 \\
\hline Schlaganfall & 0,1 & 0,2 & 0,5 & 0,3 & 1,0 & 1,2 & 3,3 & 7,4 & 15,5 & 32,1 \\
\hline \multicolumn{11}{|l|}{ Bösartige Neubildungen: } \\
\hline Dickdarm & 0,0 & 0,2 & 0,1 & 0,9 & 0,9 & 2,4 & 4,9 & 10,1 & 21,7 & 40,7 \\
\hline \multicolumn{11}{|l|}{ o Geschlechtsorgane } \\
\hline Prostata & 0,0 & 0,0 & 0,0 & 0,0 & 0,0 & 0,3 & 0,6 & 3,4 & 11,2 & 28,0 \\
\hline Gallenblase/ Pankreas & 0,0 & 0,1 & 0,3 & 0,5 & 1,0 & 2,1 & 3,0 & 3,0 & 3,3 & 5,8 \\
\hline Arthrose & 0,0 & 0,0 & 0,0 & 0,0 & 0,0 & 0,0 & 0,0 & 0,0 & 0,0 & 0,0 \\
\hline Depression & 0,0 & 0,0 & 0,0 & 0,0 & 0,0 & 0,0 & 0,0 & 0,0 & 0,0 & 0,0 \\
\hline Schlafapnoe & 0,0 & 0,0 & 0,0 & 0,0 & 0,0 & 0,0 & 0,1 & 0,1 & 0,2 & 0,1 \\
\hline Adipositas & 0,0 & 0,0 & 0,1 & 0,2 & 0,6 & 0,8 & 1,3 & 1,6 & 2,3 & 2,2 \\
\hline
\end{tabular}

Quelle: Statistisches Bundesamt 2006: www.gdb.de Ermittlung aus Ad-hoc-Tabellen (08.05.2008) 
Daten zur Berechnung der indirekten Kosten aufgrund von Mortalität

Sterbefälle je 100.000 Einwohner (2003), weibliche Bevölkerung nach Erkrankung (ICD 10) und Altersgruppen

\begin{tabular}{|c|c|c|c|c|c|c|c|c|c|c|}
\hline \multicolumn{11}{|l|}{ weibliche Bevölkerung } \\
\hline Erkrankung/ Altersgruppe & $15-20$ & $20-25$ & $25-30$ & $30-35$ & $35-40$ & $40-45$ & $45-50$ & $50-55$ & $55-60$ & $60-65$ \\
\hline Hypertonie & 0,0 & 0,1 & 0,0 & 0,1 & 0,3 & 0,6 & 1,8 & 2,9 & 4,6 & 7,7 \\
\hline Diabetes mellitus & 0,1 & 0,1 & 0,3 & 0,3 & 0,4 & 1,0 & 2,7 & 4,3 & 6,6 & 11,6 \\
\hline KHK & 0,1 & 0,6 & 0,9 & 2,0 & 3,1 & 6,8 & 12,4 & 23,7 & 40,7 & 76,7 \\
\hline Schlaganfall & 0,1 & 0,1 & 0,2 & 0,3 & 0,9 & 0,9 & 1,7 & 3,2 & 5,4 & 13,6 \\
\hline \multicolumn{11}{|l|}{ Bösartige Neubildungen: } \\
\hline Dickdarm & 0,0 & 0,1 & 0,3 & 0,4 & 1,1 & 1,9 & 4,2 & 8,7 & 15,9 & 22,9 \\
\hline i Geschlechtsorgane & 0,1 & 0,3 & 0,4 & 2,2 & 3,4 & 7,6 & 11,8 & 19,2 & 27,5 & 43,3 \\
\hline \multicolumn{11}{|l|}{ Prostata } \\
\hline Gallenblase/ Pankreas & 0,0 & 0,1 & 0,1 & 0,2 & 0,4 & 0,5 & 0,9 & 1,1 & 1,4 & 1,7 \\
\hline Arthrose & 0,0 & 0,0 & 0,0 & 0,0 & 0,0 & 0,0 & 0,0 & 0,0 & 0,0 & 0,2 \\
\hline Depression & 0,0 & 0,0 & 0,0 & 0,0 & 0,0 & 0,0 & 0,0 & 0,1 & 0,1 & 0,0 \\
\hline Schlafapnoe & 0,0 & 0,0 & 0,0 & 0,0 & 0,0 & 0,0 & 0,0 & 0,0 & 0,0 & 0,0 \\
\hline Adipositas & 0,0 & 0,0 & 0,2 & 0,1 & 0,3 & 0,7 & 0,9 & 0,8 & 1,8 & 1,8 \\
\hline
\end{tabular}

Quelle: Statistisches Bundesamt 2006: www.gdb.de Ermittlung aus Ad-hoc-Tabellen (08.05.2008) 
Daten zur Berechnung der indirekten Kosten aufgrund von Mortalität

Einwohnerzahl 2003 zum 31.12.2003 nach Altersgruppe (Erwerbsfähige)

\begin{tabular}{|c|c|c|c|c|c|c|c|c|c|c|c|}
\hline Altersgruppe & $15-20$ & $20-25$ & $25-30$ & $30-35$ & $35-40$ & $40-45$ & $45-50$ & $50-55$ & $55-60$ & $60-65$ & Summe \\
\hline männliche Bevölkerung & 2.433 .000 & 2.467 .200 & 2.393 .000 & 2.893 .500 & 3.662 .800 & 3.567 .800 & 3.049 .900 & 2.760 .100 & 2.202 .600 & 2.690 .600 & 28.120 .500 \\
\hline Weibliche Bevölkerung & 2.308 .800 & 2.403 .300 & & 2.763 .300 & 3.460 .600 & 3.409 .100 & 2.962 .700 & & 2.214 .500 & 2.785 .800 & 9.500 \\
\hline Summe & 4.741 .800 & 4.870 .500 & 4.703 .900 & 5.656 .800 & 7.123 .400 & 6.976 .900 & 6.012 .600 & 5.520 .600 & 4.417 .100 & 5.476 .400 & 55.500 .000 \\
\hline
\end{tabular}

Quelle: Statistisches Bundesamt 2005: Bevölkerungsfortschreibung 2003

\section{Erwerbstätigenquote 2003, Angaben in Prozent}

\begin{tabular}{|c|c|c|c|c|c|c|c|c|c|c|}
\hline Altersgruppe & $15-20$ & $20-25$ & $25-30$ & $30-35$ & $35-40$ & $40-45$ & $45-50$ & $50-55$ & $55-60$ & $60-65$ \\
\hline männliche Bevölkerung & 29,9 & 63,9 & 75,3 & 85,7 & 87,6 & 86,9 & 85,2 & 80,9 & 68,9 & 31,1 \\
\hline Weibliche Bevölkerung & 24,2 & 60,4 & 68,1 & 71,4 & 72,4 & 74,9 & 74,1 & 67,5 & 51,0 & 15,9 \\
\hline
\end{tabular}

Quelle: Bundesministerium für Gesundheit, Statistisches Taschenbuch 2005

Überlebensquote 2003, nach Altersgruppen (Berechnet als Mittelwert aus dem Anteil an Überlebensjahren pro Altersgruppe) Angaben in Prozent

\begin{tabular}{|l|r|r|r|r|r|r|r|r|r|r|}
\hline Altersgruppe & \multicolumn{1}{|c|}{$\mathbf{1 5 - 2 0}$} & \multicolumn{1}{|c|}{$\mathbf{2 0 - 2 5}$} & \multicolumn{1}{|c|}{$\mathbf{2 5 - 3 0}$} & $\mathbf{3 0 - 3 5}$ & $\mathbf{3 5 - 4 0}$ & $\mathbf{4 0 - 4 5}$ & $\mathbf{4 5 - 5 0}$ & \multicolumn{1}{|c|}{$\mathbf{5 0 - 5 5}$} & $\mathbf{5 5 - 6 0}$ & $\mathbf{6 0 - 6 5}$ \\
\hline männliche Bevölkerung & 99,2 & 98,9 & 98,5 & 98,1 & 97,6 & 96,8 & 95,3 & 93,0 & 89,8 & 84,9 \\
\hline Weibliche Bevölkerung & 99,4 & 99,3 & 99,1 & 99,0 & 98,7 & 98,2 & 97,5 & 96,3 & 94,5 & 92,0 \\
\hline
\end{tabular}

Quelle: Statistisches Jahrbuch 2006 für die Bundesrepublik Deutschland: Sterbetafeln (2002/2004), Absterbeordnung

Berechnung: (niedrigster Wert Altersgruppe + höchster Wert Altersgruppe) : $2=[X+(X+5)]: 2$ (angegeben in Prozent) 


\section{Berechnung der verlorenen Erwerbsjahre aufgrund von Mortalität}

\section{Annahmen:}

Es existieren keine spezifischen personellen Unterschiede oder Risiken in der Bevölkerung außer Alter und Geschlecht.

Jede Person innerhalb einer Altersgruppe weist eine identische Überlebensquote und eine identische Erwerbstätigkeitsquote auf.

Gleichverteilung der Todesfälle innerhalb der jeweiligen Altersgruppe

Alle Werte in der Zukunftssicht bleiben konstant.

Die Lebenserwartung bleibt unberücksichtigt, da sie durchweg höher liegt als das höchste Erwerbsalter von 65 Jahren.

\section{Vorgehen:}

1.) Ermittlung der Berechnungsbasis über Einwohnerzahl $x$ Überlebensquote $x$ Erwerbsquote je Altersgruppe und Geschlecht

2.) Ergebnis aus 1.) $x$ absolute Sterbefälle pro Erkrankung je Altersgruppe und Geschlecht

3.) Berechnung von verlorenen Erwerbsjahren je Altersgruppe und Geschlecht $=$ (niedrigster Wert + höchster Wert je Altersgruppe) $: 2=[X+(X+5)]: 2$ Berechnungsschritt 1.) Ermittlung der Einwohnerzahl, gewichtet mit Überlebensquote und Erwerbsquote je Altersgruppe und Geschlecht

\begin{tabular}{|c|c|c|c|c|c|c|c|c|c|c|}
\hline ännliche & & & & & & & & & & \\
\hline Altersgruppe & $15-20$ & $20-25$ & $25-30$ & \begin{tabular}{|l|}
$30-35$ \\
\end{tabular} & \begin{tabular}{|l|}
$35-40$ \\
\end{tabular} & \begin{tabular}{|l|}
$40-45$ \\
\end{tabular} & \begin{tabular}{|l|}
$45-50$ \\
\end{tabular} & $50-55$ & $55-60$ & $60-65$ \\
\hline Gesamt & 2.433 .000 & 2.467 .200 & 2.393 .000 & 2.893 .500 & 3.662 .800 & 3.567 .800 & 3.049 .900 & 2.760 .100 & 2.202 .600 & 2.690 .600 \\
\hline Überlebensquote in \% & 99,2 & 98,9 & 98,5 & 98,1 & 97,6 & 96,8 & 95,3 & 93,0 & 89,8 & 84,9 \\
\hline Überlebensquote Faktor & 0,992 & 0,989 & 0,985 & 0,981 & 0,976 & 0,968 & 0,9 & 0,930 & 0,898 & 0,849 \\
\hline Erwerbstätigenquo & 29,9 & 63,9 & 75,3 & & 87,6 & 86,9 & 85,2 & 80,9 & 68,9 & 31,1 \\
\hline Erwerbstätigenquote & & & & & & & & & & \\
\hline Faktor & 0,299 & 0,639 & 0,753 & 0,857 & 0,876 & 0,869 & 0,852 & 0,809 & 0,689 & 0,311 \\
\hline relevanter Anteil & 721.647 & 1.559 .199 & 1.774 .900 & 2.432 .615 & 3.131 .606 & 3.001.205 & 2.476.385 & 2.076 .616 & 1.362 .797 & 710.423 \\
\hline
\end{tabular}

\begin{tabular}{|c|c|c|c|c|c|c|c|c|c|c|}
\hline aihliche & & & & & & & & & & \\
\hline Altersgruppe & $15-20$ & \begin{tabular}{|l|}
$20-25$ \\
\end{tabular} & $25-30$ & $30-35$ & \begin{tabular}{|l|}
$35-40$ \\
\end{tabular} & $40-45$ & \begin{tabular}{|l|}
$45-50$ \\
\end{tabular} & $50-55$ & $55-60$ & $60-65$ \\
\hline Gesamt & 2.308 .800 & 2.403 .300 & 2.310 .900 & 2.763 .300 & 3.460 .600 & 3.409 .100 & 2.962 .700 & 2.760 .500 & 2.214 .500 & 2.785 .800 \\
\hline Überlebensquote in \% & 99,4 & 99,3 & 99,1 & 99,0 & 98,7 & 98,2 & 97,5 & 96,3 & 94,5 & 92,0 \\
\hline Überlebensquote Faktor & 0,994 & 0,993 & 0,991 & 0,990 & 0,987 & 0,982 & 0,975 & 0,963 & 0,945 & 0,920 \\
\hline Erwerbstätigenquote in \% & 24,2 & 60,4 & 3,1 & 71,4 & 72,4 & 74,9 & 74,1 & 67,5 & 51,0 & 15,9 \\
\hline Erwerbstätigenquote & & & & & & & & & & \\
\hline$\frac{\text { Faktor }}{\text { relevanter Anteil }}$ & $\begin{array}{r}0,242 \\
555.377\end{array}$ & \begin{tabular}{|r|}
0,604 \\
1.441 .432 \\
\end{tabular} & \begin{tabular}{|r|}
, 681 \\
1.559 .559 \\
\end{tabular} & \begin{tabular}{r|}
$0, / 14$ \\
1.953 .266 \\
\end{tabular} & \begin{tabular}{|r|}
,$/ 224$ \\
2.472 .903 \\
\end{tabular} & \begin{tabular}{|r|}
$0, / 49$ \\
2.507 .454 \\
\end{tabular} & \begin{tabular}{|r|}
, 141 \\
2.140 .477 \\
\end{tabular} & \begin{tabular}{|r|}
$0,6 / 5$ \\
1.794 .394 \\
\end{tabular} & \begin{tabular}{|r|}
0,510 \\
1.067 .278 \\
\end{tabular} & $\begin{array}{r}0,159 \\
407.507\end{array}$ \\
\hline
\end{tabular}

Quelle: Eigene Berechnungen auf Basis der angegebenen Datenquellen. 
Berechnungsschritt 2.) Anzahl der Sterbefälle (2003), nach Erkrankung (ICD 10) und Altersgruppen unter Berücksichtigung von Erwerbstätigenquote und Überlebensquote

\begin{tabular}{|c|c|c|c|c|c|c|c|c|c|c|}
\hline \multicolumn{11}{|l|}{ männliche Bevölkerung } \\
\hline Erkrankung/ Altersgruppe & $15-20$ & $20-25$ & $25-30$ & $30-35$ & $35-40$ & $40-45$ & $45-50$ & $50-55$ & $55-60$ & $60-65$ \\
\hline Hypertonie & 0 & 2 & 2 & 2 & 31 & 39 & 92 & 131 & 134 & 120 \\
\hline Diabetes mellitus & 1 & 3 & 7 & 22 & 38 & 87 & 136 & 210 & 217 & 204 \\
\hline KHK & 9 & 22 & 51 & 102 & 332 & 780 & 1355 & 1971 & 2125 & 1890 \\
\hline Schlaganfall & 1 & 3 & 9 & 7 & 31 & 36 & 82 & 154 & 211 & 228 \\
\hline \multicolumn{11}{|l|}{ Bösartige Neubildungen: } \\
\hline Dickdarm & 0 & 3 & 2 & 22 & 28 & 72 & 121 & 210 & 296 & 289 \\
\hline \multicolumn{11}{|l|}{ o Geschlechtsorgane } \\
\hline Prostata & 0 & 0 & 0 & 0 & 0 & 9 & 15 & 71 & 153 & 199 \\
\hline Gallenblase/ Pankreas & 0 & 2 & 5 & 12 & 31 & 63 & 74 & 62 & 45 & 41 \\
\hline Arthrose & 0 & 0 & 0 & 0 & 0 & 0 & 0 & 0 & 0 & 0 \\
\hline Depression & 0 & 0 & 0 & 0 & 0 & 0 & 0 & 0 & 0 & 0 \\
\hline Schlafapnoe & 0 & 0 & 0 & 0 & 0 & 0 & 2 & 2 & 3 & 1 \\
\hline Adipositas & 0 & 0 & 2 & 5 & 19 & 24 & 32 & 33 & 31 & 16 \\
\hline \multicolumn{11}{|l|}{ weibliche Bevölkerung } \\
\hline Erkrankung/ Altersgruppe & $15-20$ & $20-25$ & $25-30$ & $30-35$ & $35-40$ & $40-45$ & $45-50$ & $50-55$ & $55-60$ & $60-65$ \\
\hline Hypertonie & 0 & 1 & 0 & 2 & 7 & 15 & 39 & 52 & 49 & 31 \\
\hline Diabetes mellitus & 1 & 1 & 5 & 6 & 10 & 25 & 58 & 77 & 70 & 47 \\
\hline $\mathrm{KHK}$ & 1 & 9 & 14 & 39 & 77 & 171 & 265 & 425 & 434 & 313 \\
\hline Schlaganfall & 1 & 1 & 3 & 6 & 22 & 23 & 36 & 57 & 58 & 55 \\
\hline \multicolumn{11}{|l|}{ Bösartige Neubildungen: } \\
\hline Dickdarm & 0 & 1 & 5 & 8 & 27 & 48 & 90 & 156 & 170 & 93 \\
\hline ㅇ Geschlechtsorgane & 1 & 4 & 6 & 43 & 84 & 191 & 253 & 345 & 294 & 176 \\
\hline \multicolumn{11}{|l|}{ Prostata } \\
\hline Gallenblase/ Pankreas & 0 & 1 & 2 & 39 & 10 & 13 & 19 & 20 & 15 & 7 \\
\hline Arthrose & 0 & 0 & 0 & 0 & 0 & 0 & 0 & 0 & 0 & 1 \\
\hline Depression & 0 & 0 & 0 & 0 & 0 & 0 & 0 & 2 & 1 & 0 \\
\hline Schlafapnoe & 0 & 0 & 0 & 0 & 0 & 0 & 0 & 0 & 0 & 0 \\
\hline Adipositas & 0 & 0 & 3 & 2 & 7 & 18 & 19 & 14 & 19 & 7 \\
\hline
\end{tabular}

Quelle: Eigene Berechnungen auf Basis der angegebenen Datenquellen. 
Berechnungsschritt 3.) Berechnung von verlorenen Erwerbsjahren je Altersgruppe und Geschlecht.

Verlorene Erwerbsjahre $=$ (niedrigster Wert je Altersgruppe + höchster Wert je Altersgruppe) $: 2=[X+(X+5)]: 2$

Z.B. 3 Todesfälle in Altersgruppe 20-25: [(Restjahre niedrigster Wert 45 Jahre + Restjahre höchster Wert $=40$ Jahre $): 2] \times 3$ Ereignisse

männliche Bevölkerung

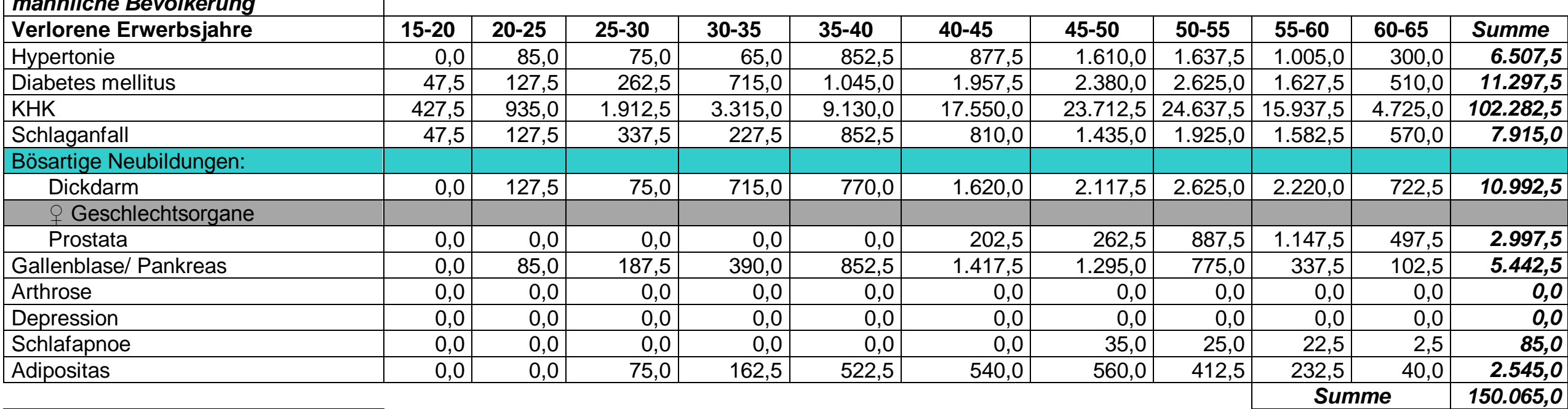

\begin{tabular}{|c|c|c|c|c|c|c|c|c|c|c|c|}
\hline & & & & & & & & & \multicolumn{2}{|c|}{ Summe } & \multirow{2}{*}{$150.065,0$} \\
\hline \multirow{2}{*}{$\begin{array}{l}\text { weibliche Bevölkerung } \\
\text { Verlorene Erwerbsjahre }\end{array}$} & & & & & & & & & & & \\
\hline & $15-20$ & $20-25$ & $25-30$ & $30-35$ & $35-40$ & $40-45$ & $45-50$ & $50-55$ & $55-60$ & $60-65$ & Summe \\
\hline Hypertonie & 0,0 & 42,5 & 0,0 & 65,0 & 192,5 & 337,5 & 682,5 & 650,0 & 367,5 & 77,5 & $2.415,0$ \\
\hline Diabetes mellitus & 47,5 & 42,5 & 187,5 & 195,0 & 275,0 & 562,5 & $1.015,0$ & 962,5 & 525,0 & 117,5 & $3.930,0$ \\
\hline KHK & 47,5 & 382,5 & 525,0 & $1.267,5$ & $2.117,5$ & $3.847,5$ & $4.637,5$ & $5.312,5$ & $3.255,0$ & 782,5 & $22.175,0$ \\
\hline Schlaganfall & 47,5 & 42,5 & 112,5 & 195,0 & 605,0 & 517,5 & 630,0 & 712,5 & 435,0 & 137,5 & $3.435,0$ \\
\hline \multicolumn{12}{|l|}{ Bösartige Neubildungen: } \\
\hline Dickdarm & 0,0 & 42,5 & 187,5 & 260,0 & 742,5 & $1.080,0$ & $1.575,0$ & $1.950,0$ & $1.275,0$ & 232,5 & $7.345,0$ \\
\hline of Geschlechtsorgane & 47,5 & 170,0 & 225,0 & $1.397,5$ & $2.310,0$ & $4.297,5$ & $4.427,5$ & $4.312,5$ & $2.205,0$ & 440,0 & $19.832,5$ \\
\hline \multicolumn{12}{|l|}{ Prostata } \\
\hline Gallenblase/ Pankreas & 0,0 & 42,5 & 75,0 & $1.267,5$ & 275,0 & 292,5 & 332,5 & 250,0 & 112,5 & 17,5 & $2.665,0$ \\
\hline Arthrose & 0,0 & 0,0 & 0,0 & 0,0 & 0,0 & 0,0 & 0,0 & 0,0 & 0,0 & 2,5 & 2,5 \\
\hline Depression & 0,0 & 0,0 & 0,0 & 0,0 & 0,0 & 0,0 & 0,0 & 25,0 & 7,5 & 0,0 & 32,5 \\
\hline Schlafapnoe & 0,0 & 0,0 & 0,0 & 0,0 & 0,0 & 0,0 & 0,0 & 0,0 & 0,0 & 0,0 & 0,0 \\
\hline Adipositas & 0,0 & 0,0 & 112,5 & 65,0 & 192,5 & 405,0 & 332,5 & 175,0 & 142,5 & 17,5 & $1.442,5$ \\
\hline \multicolumn{11}{|l|}{ Quelle: Eigene Berechnung } & $63.275,0$ \\
\hline
\end{tabular}




\section{Barwertfaktoren}

Basis: Durchschnittlich verlorene Erwerbsjahre bis 65 Jahre je Altersgruppe; durchschnittliches Bruttojahreinkommen (Voll- und Teilzeit) 27.493 $€$ (2003)

\begin{tabular}{|c|c|c|c|c|c|c|c|c|c|c|}
\hline Altersgruppe & $15-20$ & $20-25$ & $25-30$ & $30-35$ & $35-40$ & $40-45$ & $45-50$ & $50-55$ & $55-60$ & $60-65$ \\
\hline$\varnothing$ der Verlustjahre bis 65 & 47,5 & 42,5 & 37,5 & 32,5 & 27,5 & 22,5 & 17,5 & 12,5 & 7,5 & 2,5 \\
\hline 4\% Diskontierungssatz & 0,1552088 & 0,1888353 & 0,2297470 & 0,2795223 & 0,3400816 & 0,4137613 & 0,5034039 & 0,6214678 & 0,7451607 & 0,9066020 \\
\hline 6\% Diskontierungssatz & 0,0628017 & 0,0840428 & 0,1124683 & 0,1505079 & 0,2014136 & 0,2695368 & 0,3607010 & 0,4826993 & 0,6459606 & 0,8644410 \\
\hline Barwert 4\% x Einkommen & $4.267,2$ & $5.191,6$ & $6.316,4$ & $7.684,9$ & $9.349,9$ & $11.375,5$ & $13.840,1$ & $17.086,0$ & $20.486,7$ & $24.925,2$ \\
\hline Barwert $6 \%$ x Einkommen & $1.726,6$ & $2.310,6$ & $3.092,1$ & $4.137,9$ & $5.537,5$ & $7.410,4$ & $9.916,8$ & $13.270,9$ & $17.759,4$ & $23.766,1$ \\
\hline
\end{tabular}

Quelle: Eigene Berechnungen auf Basis der angegebenen Datenquellen. 
Diskontierungssatz von 4\%

—313.290,00€; $2,41 \%$

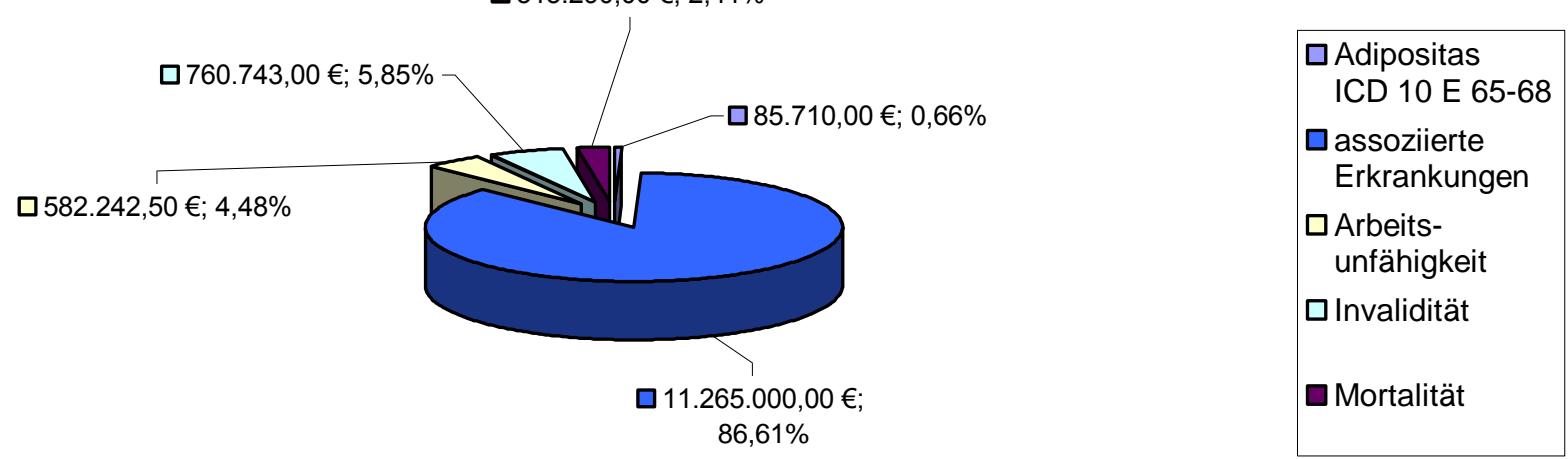

Diskontierungssatz von $6 \%$

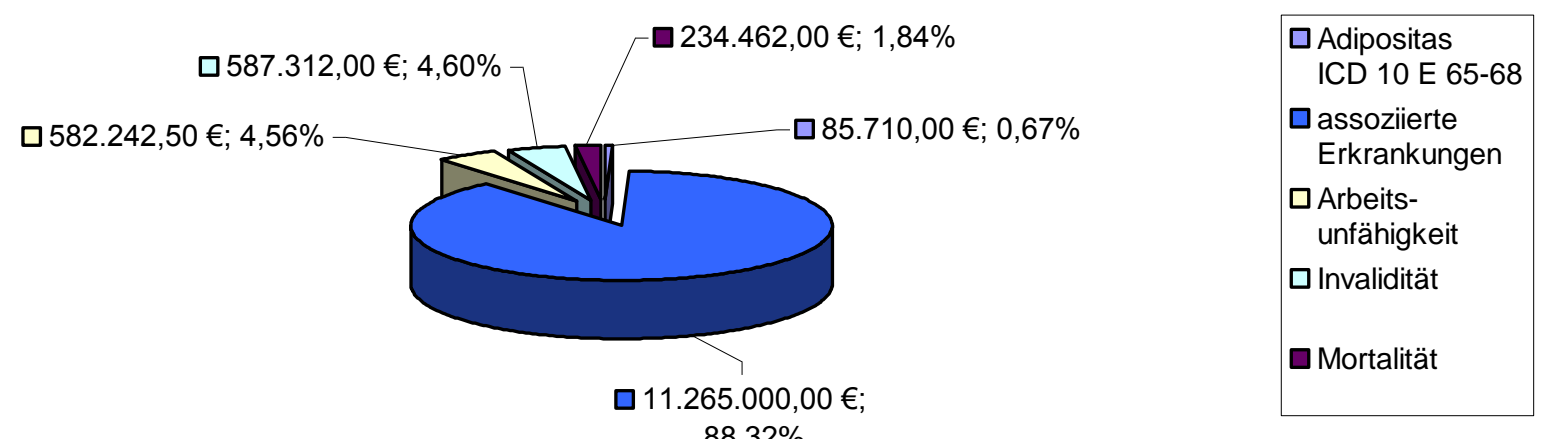




\section{Danksagung}

Mein besonderer Dank gilt meinem Doktorvater Herrn Univ.-Prof. Dr. Hans Hauner, Ordinarius des Lehrstuhls für Ernährungsmedizin der Technischen Universität München, für die Initiierung und unkomplizierte Betreuung der Dissertation mit einem hochaktuellen Thema in der Schnittstelle zwischen Medizin und Ökonomie.

Darüber hinaus danke ich meinen Eltern sowie meinen Freunden und Kollegen, die mich mit Diskussionen, Vorschlägen und wertvollen Anregungen unterstützt haben.

Zudem gilt der Dank den Mitarbeitern der kontaktierten Kliniken, öffentlichen Einrichtungen, Krankenkassen und Firmen, die meine Anfragen in kollegialer und freundlicher Weise bearbeitet und mir erforderliches Datenmaterial zur Verfügung gestellt haben.

Dr. Klaus-Peter Knoll

Herbst 2009 